\title{
ZOOTAXA
}

3084

\section{Revision of the Western Palearctic Meteorini (Hymenoptera, Braconidae), with a molecular characterization of hidden Fennoscandian species diversity}

\author{
JULIA STIGENBERG \& FREDRIK RONQUIST \\ Department of Zoology, Stockholm University and Department of Entomology, Swedish Museum of Natural History, Box 50007, \\ SE-10405 Stockholm, Sweden; e-mail: julia.stigenberg@nrm.se \\ Department of Biodiversity Informatics, Swedish Museum of Natural History, Box 50007, SE-10405 Stockholm, Sweden; \\ e-mail:fredrik.ronquist@nrm.se
}

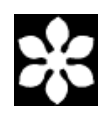

Magnolia Press

Auckland, New Zealand 
JULIA STIGENBERG \& FREDRIK RONQUIST

Revision of the Western Palearctic Meteorini (Hymenoptera, Braconidae), with a molecular characterization of hidden Fennoscandian species diversity

(Zootaxa 3084)

95 pp.; $30 \mathrm{~cm}$.

28 Oct. 2011

ISBN 978-1-86977-817-0 (paperback)

ISBN 978-1-86977-818-7 (Online edition)

FIRST PUBLISHED IN 2011 BY

Magnolia Press

P.O. Box 41-383

Auckland 1346

New Zealand

e-mail: zootaxa@mapress.com

http://www.mapress.com/zootaxa/

(C) 2011 Magnolia Press

All rights reserved.

No part of this publication may be reproduced, stored, transmitted or disseminated, in any form, or by any means, without prior written permission from the publisher, to whom all requests to reproduce copyright material should be directed in writing.

This authorization does not extend to any other kind of copying, by any means, in any form, and for any purpose other than private research use.

ISSN 1175-5326 (Print edition)

ISSN 1175-5334 (Online edition) 


\title{
Table of contents
}

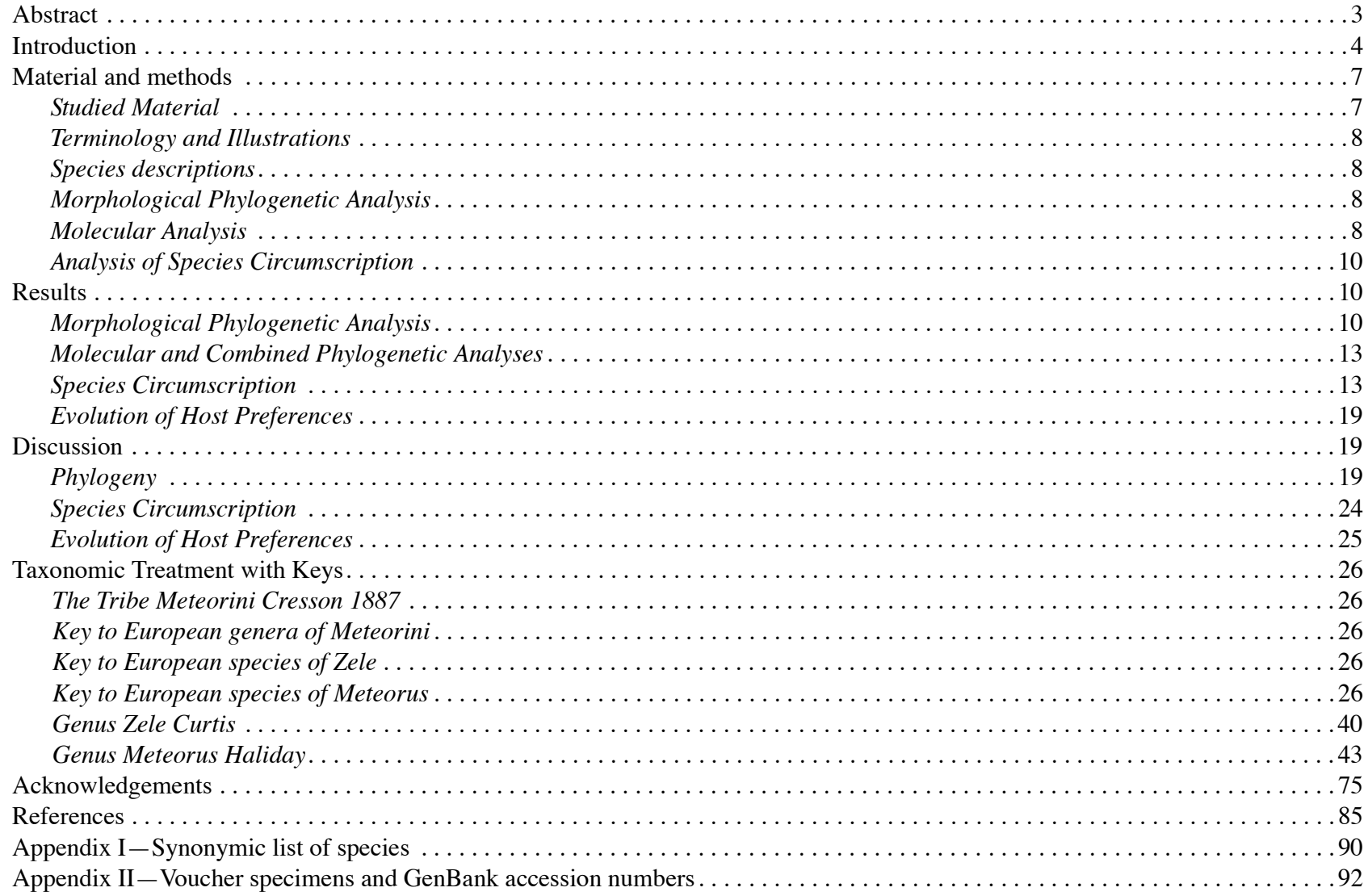

\begin{abstract}
The tribe Meteorini includes two genera, Meteorus and Zele, which are koinobiont endoparasitoids of larval Lepidoptera and Coleoptera. More than 300 species are known, about one fifth of which occur in the Western Palearctic. Here, we revise the Western Palearctic species, based partly on traditional approaches and partly on molecular analysis of recent Swedish and Finnish material. For the analyses of phylogenetic relationships and cryptic species diversity, we coded 17 morphological characters and sequenced two markers, 28S D2 (649 bp) and CO1 (665 bp). More than 1970 specimens representing 54 species of Meteorus Haliday and 5 species of Zele Curtis were studied; of these, 177 specimens representing 41 species were sequenced. Seven new species are described, all from the Fennoscandian material: Meteorus artocercus $\mathbf{s p .}$ nov., M. densipilosus $\mathbf{s p . ~ n o v . , ~ M . ~ e k l u n d i ~} \mathbf{s p . ~ n o v . , ~ M . ~ l o n g i p i l o s u s ~} \mathbf{s p . ~ n o v . , ~ M . ~ s i b y l l a e ~} \mathbf{s p}$. nov., M. stenomastax sp. nov., and $M$. subtilisulcus sp. nov. Four new synonyms are introduced: Z. chlorophthalmus (Spinola 1808), syn. nov. for M.pallidus (Nees 1812), M. punctifrons Thomson 1895, syn. nov. for M. varinervis (Tobias 1986), M. melanostictus Capron 1887, syn. nov for M. monachae (Tobias 1986), and M. tenellus Marshall 1887, syn. nov. for M. boreus (Tobias 1986). Meteorus tenellus is removed from synonymy with M. cinctellus. Sequence analysis indicated the presence of at least 12 additional cryptic species but these cannot be separated morphologically at this point and, therefore, we do not describe them here. The phylogenetic results suggest that Zele should be included within Meteorus but we refrain from formal changes of the generic classification until more comprehensive phylogenetic analyses of the tribe can be completed. A key to the known Western Palearctic species is presented.
\end{abstract}

Key words: parasitic wasps, molecular taxonomy, cryptic species, general mixed Yule coalescent, evolution, host preferences, identification keys 


\section{Introduction}

The Meteorini (Cresson 1887) are small to medium size $(2-10 \mathrm{~mm})$ euphorine braconids, classified into two genera: the species-rich and cosmopolitan Meteorus Haliday (1832) and the small Holarctic and Neotropical Zele Curtis (1835). After accounting for the new species described herein, the two genera together include 337 species in the world (308 Meteorus and 29 Zele, Yu et al. 2005, Shaw et al. 2009, Aguirre et al. 2010, 2011), 59 of which occur in the Western Palearctic (54 Meteorus and 5 Zele) (Appendix I).

Unlike other euphorine braconids that largely attack adult insects, the Meteorini target Lepidoptera and Coleoptera larvae. The host larvae are typically hidden under bark, in wood, or in fungi (Maeto 1990a, Shaw et al. 1991). Most meteorines are solitary parasitoids but some species are gregarious. They attack their hosts in the same way as most other euphorines, namely by bending the abdomen under the thorax and pushing the ovipositor out so that it projects forwards, in front of the wasp. They seem to preferably attack mobile host larvae (Parker 1931, Tobias 1966). Oviposition occurs in young host larvae, while they are exposed, and development is completed before the host enters the pupal stage.

Members of Meteorus are either univoltine or multivoltine. Some species produce two complete generations upon one brood of host larvae, the first being upon very young individuals and the second upon those that are nearly mature (Clausen 1940). The solitary suspended cocoon made by some Meteorus larvae is unique amongst the Braconidae and only the Campopleginae amongst the Ichneumonidae have members that construct similar cocoons. No other hymenopterans are known to form suspended cocoons (Zitani 2003). The suspended cocoons are fully exposed whilst the non-suspended cocoons often are placed in a concealed environment.

Members of Zele seem to be solitary parasitoids. In Britain, at least one species is noted to be bivoltine whilst others are univoltine (Shaw \& Huddleston 1991). They overwinter as prepupae in a fusiform and felted cocoon in the pupation chamber of the host. Zele adults are predominantly crepuscular or nocturnal and are also attracted to light (Huddleston and Gauld 1988). In both genera, the adult emerges from the cocoon by cutting the cap off of its anterior end.

The circumscription of the Meteorini has been a subject of controversy for some time (van Achterberg 1979, Huddleston 1980, Zitani 2003). Mason (1973), in his paper on the identity of Zele (treated by him under the junior synonym Zemiotes Förster), proposed that the Meteorini formed an unnatural assemblage and that Zele was more closely related to Homolobus Förster (subfam. Homolobinae) than to Meteorus. Mason based this hypothesis on the following similarities shared between Homolobus and Zele: (1) radiellan cell broadening apically and divided by a transverse vein; (2) tarsal claws with a large basal tooth; (3) apical half or more of abdominal tergites 3-6 uniformly hairy, never with only a single, subapical, transverse row on each tergite; and (4) the cocoon covered with fine, fluffy witish silk and never suspended on a cord or covered in coarse brown silk as in Meteorus. In his revision of the subfamily Zelinae, van Achterberg (1979) redefined Zele and moved it to the tribe Meteorini of the subfamily Euphorinae. He thoroughly studied the similarities between Zele and Homolobus listed by Mason (1973), as well as other characters potentially informative about the placement of Zele, and came to the conclusion that Zele is not the sister group to Homolobus but instead is likely to be the sister group to Meteorus.

Maeto (1990a) proposed two putative morphological synapomorphies for (Meteorus + Zele): (1) the median longitudinal carinae of the propodeum divided just before the orifice, forming a subtriangular cell; and (2) eyes distinctly setose. The monophyly of (Meteorus + Zele) was also strongly supported in the more extensive morphological phylogenetic analysis by Zitani (2003) and has been confirmed in several molecular analyses as well (Li et al. 2003, Shi et al. 2005, Pitz et al. 2007). We consider the monophyly of (Meteorus + Zele) well established today.

The Meteorini (Meteorus + Zele) are considered as a tribe within Euphorinae by some workers (Muesebeck 1923, Maeto 1986a, van Achterberg 1979 and 1984, Tobias 1986, Li et. al. 2003) and as a separate subfamily by others (Foerster 1862, Shaw 1985, Maeto 1990a, Zitani 2003, Boring 2010). Based on morphology, Tobias (1966) placed Meteorus basally within the Euphorinae, without any comments on the position of Zele. The molecular analyses by Li et al. (2003) and Shi et al. (2005) support the view that the Meteorini are the sister group of other Euphorinae.

If the sister-group hypothesis is correct, then the Meteorini could in principle be treated as a subfamily distinct from the Euphorinae. However, we treat the group as a tribe within the Euphorinae for several reasons. First, we consider it well established that the Meteorini and other Euphorinae form a monophyletic group. Second, we think 
it is impractical to recognize such a small group as the Meteorini as a subfamily, since many of the other braconid subfamilies are much larger and more diverse. Finally, we do not yet consider it certain that the Meteorini are placed phylogenetically outside the remaining Euphorinae. Some recent analyses (Dowton et al. 1998, 2002; Pitz et al. 2007) do indeed place them as sister to the rest of the Euphorinae, but others suggest they may be more deeply nested within the Euphorinae (Belshaw et al. 2000; Belshaw and Quicke 2002; Li et al. 2003; Shi et al. 2005).

Three morphological or biological synapomorphies have been proposed for Euphorinae including Meteorini (van Achterberg 1984): (1) loss of the final ectoparasitic phase in larval development; (2) smooth toothless larval mandibles; and (3) development of parasitism of adults. Parasitism of adults is a doubtful synapomorphy at this level, however, since the Meteorini are larval parasitoids; it appears more likely to be a synapomorphy of the Euphorinae excluding the Meteorini. Monophyly of Euphorinae excluding Meteorini is also evidenced by two additional morphological characters: (1) reduction of the maxillary palp to five segments or less; and (2) strong curvature of the apical radial segment of the fore wing (Shaw 1985). The maxillary palp is six-segmented in Meteorus and Zele, and this is likely to be the plesiomorphic state for the Euphorinae.

Members of Zele are united by several distinct synapomorphies, including the scattered tergal setae, the large second submarginal cell of the fore wing, and the apically widened and transversely subdivided marginal cell of the hind wing (Maeto 1990a). However, it has been difficult to find morphological synapomorphies for the genus Meteorus, suggesting that Meteorus might be paraphyletic with respect to Zele. In his systematic studies of the Meteorini of Japan, Maeto (1989a, 1990a) recognized seven species groups of Meteorus based on morphology and biology (Table 1). He specifically lists two structural synapomorphies that support the hypothesis that members of Zele form a monophyletic group with only some clades (the ictericus, rubens, and pulchricornis groups; see Table 1 for details) within Meteorus: (1) tarsal claws with a submedial lobe; and (2) large ocelli. He suggested that the resulting taxonomic problem, a paraphyletic Meteorus, be solved by rearranging Meteorus into several monophyletic genera. However, he argued that such a rearrangement should wait until a more extensive sample of the world fauna has been analyzed phylogenetically.

Using morphological characters from 16 species of Meteorus and two species of Zele, Zitani (2003) analyzed the relationships among species of Meteorus and between Meteorus and Zele (Fig. 1). The species were chosen to represent the species groups of Maeto. In total, 32 adult morphological characters and 7 cocoon characters were used. In contrast to the analysis of Maeto, Zitani's results indicated that: (1) Zele and Meteorus are reciprocally monophyletic groups; and (2) Zele is the sister group to Meteorus. However, the monophyly of Meteorus and Zele received only moderately high bootstrap support values (81\% and $74 \%$, respectively).

Huddleston (1980) presented a key to all Western Palaearctic species of Meteorus known at the time. He also provided good species descriptions. There are additional keys to species of Meteorus for Japan (Maeto 1986a, b, 1988a, b, 1989a, b, 1990b), for China (Chen \& Wu 2000), for Northern America (Muesebeck 1923), for the European part of Russia (Tobias 1986), for the Russian Far East (Belokobylskij 2000), for Columbia (Aguirre et al. 2011) and for Australia and New Guinea (Huddleston 1983). However, modern keys to the Western Palearctic fauna of Meteorus are missing. The Western Palearctic species of Zele were keyed by van Achterberg $(1979,1984)$.

In this paper, we revise the Western Palearctic species of Meteorini based partly on study of types and other material available in major natural history collections, and partly on morphological and molecular analysis of recent Fennoscandian material. The latter material comes mostly from the Swedish Malaise Trap Project (SMTP), a comprehensive three-year inventory of the Swedish insect fauna using Malaise traps (Karlsson et al. 2005), but also from Finland. More than 1900 specimens were studied in total. We describe seven new species from the Fennoscandian material and present molecular evidence for the existence of at least 12 additional cryptic species. We have not been able to distinguish the latter morphologically, and refrain from describing them here. The phylogenetic results are used to examine the monophyly of Meteorus and Zele and to examine the evolution of host preferences. We conclude the paper with a key to the known Western Palearctic species of the tribe, particularly targeted at non-specialists. The key is shorter, more concise, and should be easier to use than the currently available ones. 
TABLE 1. Maeto's (1989a, 1990a) classification of Meteorus into species groups.

\begin{tabular}{|c|c|c|}
\hline Species group & $\begin{array}{l}\text { Meteorus species placed in the } \\
\text { group }\end{array}$ & Characteristics of group \\
\hline corax group & corax, sulcatus & $\begin{array}{l}\text { Petiolar tergum broad at base; deep and large dorsope; }+ \\
\text { antennae subapically bearing a ventral field of densely } \\
\text { placed basiconic pegs. }\end{array}$ \\
\hline hirsutipes group & $\begin{array}{l}\text { angustatus, cespitator, cis, eadyi, } \\
\text { filator, gotoi, hirsutipes, jaculator, } \\
\text { jezoensis, kyushuensis, longi- } \\
\text { caudis, obfuscatus, vexator }\end{array}$ & $\begin{array}{l}\text { Shape of pterostigma broad with the vein } r \text { originating near } \\
\text { middle; inwardly curved striations on petiolar tergum. }\end{array}$ \\
\hline albizonalis group & $\begin{array}{l}\text { abdominator, acutus, albizonalis, } \\
\text { consimilis, curvus, punctifrons }\end{array}$ & $\begin{array}{l}\text { Ovipositor distinctly curved ventrad; vein } 3 \text {-SR of fore wing } \\
\text { very short; pterostigma not broad and vein } r \text { originating } \\
\text { beyond the middle of it. }\end{array}$ \\
\hline micropterus group & micropterus, salicorniae & $\begin{array}{l}\text { Face strongly convex; medio-dorsally interrupted occipital } \\
\text { carina; } 4^{\text {th }} \text { antennal article on } q \text { stout; hind femur stout; } \\
\text { propodeum without median longitudinal carina. }\end{array}$ \\
\hline $\begin{array}{l}\text { pulchricornis group } \\
\text { (subgroups: pulchricornis, nodai, } \\
\text { pendulus, colon, versicolor) }\end{array}$ & & Slender and strongly twisted mandibles. \\
\hline subgroup: pulchricornis & $\begin{array}{l}\text { abscissus, communis, dimidiatus, } \\
\text { hyphantriae lionotus, proximus, } \\
\text { pulchricornis, unicolor }\end{array}$ & $\begin{array}{l}\text { Clypeus with a dense pile of erect hairs; median tubercle of } \\
\text { frons absent; ventral borders of } 1^{\text {st }} \text { tergum joined in the mid- } \\
\text { dle of the segment or narrowly separated. }\end{array}$ \\
\hline subgroup nodai & nodai & $\begin{array}{l}\text { Ocelli very large; OOL } / \mathrm{OD}=0.5-0.7 \text {; median tubercle of } \\
\text { frons absent; } 1^{\text {st }} \text { tergum without dorsope; the ventral borders } \\
\text { joined at the basal } 1 / 3 \text {. }\end{array}$ \\
\hline subgroup pendulus & pendulus, limbatus, naranage & $\begin{array}{l}\text { Median tubercle of frons absent; } 1^{\text {st }} \text { tergum with deep dor- } \\
\text { sope; ventral borders separated. }\end{array}$ \\
\hline subgroup colon & $\begin{array}{l}\text { melanostictus, colon, cinctellus, } \\
\text { watanabei }\end{array}$ & $\begin{array}{l}\text { Median tubercle of frons present; vein m-cu of fore wing } \\
\text { usually postfurcal to vein } 2-\mathrm{SR} ; 1^{\text {st }} \text { tergum without dorsope } \\
\text { (except melanostictus) }\end{array}$ \\
\hline subgroup versicolor & $\begin{array}{l}\text { versicolor, takenoi, albifasciatus, } \\
\text { camptolomae, kurokoi, obsoletus, } \\
\text { insulicola }\end{array}$ & $\begin{array}{l}\text { Median tubercle of frons absent; } 1^{\text {st }} \text { tergum without dorsope; } \\
\text { the ventral borders joined from the base to its basal } 2 / 5 \text { or } 1 / 2\end{array}$ \\
\hline ictericus group & $\begin{array}{l}\text { affinis, campestris, endoclytae, } \\
\text { graciliventris, ictericus, ruficeps, } \\
\text { tabidus, trachynotus }\end{array}$ & $\begin{array}{l}\text { Large ocelli; mandibles stout and moderately twisted; ovi- } \\
\text { positor long and straight; eyes moderately or strongly con- } \\
\text { vergent ventrad; tarsal claws usually with a submedial lobe. }\end{array}$ \\
\hline rubens group & heliophilus, rubens & $\begin{array}{l}\text { Second valvula swollen apically and with a minute subapical } \\
\text { notch; large ocelli; short ovipositor; stout and moderately } \\
\text { twisted mandibles or strongly twisted in the apical portion; } \\
\text { gregarious parasitoids with unsuspended cocoons. }\end{array}$ \\
\hline
\end{tabular}




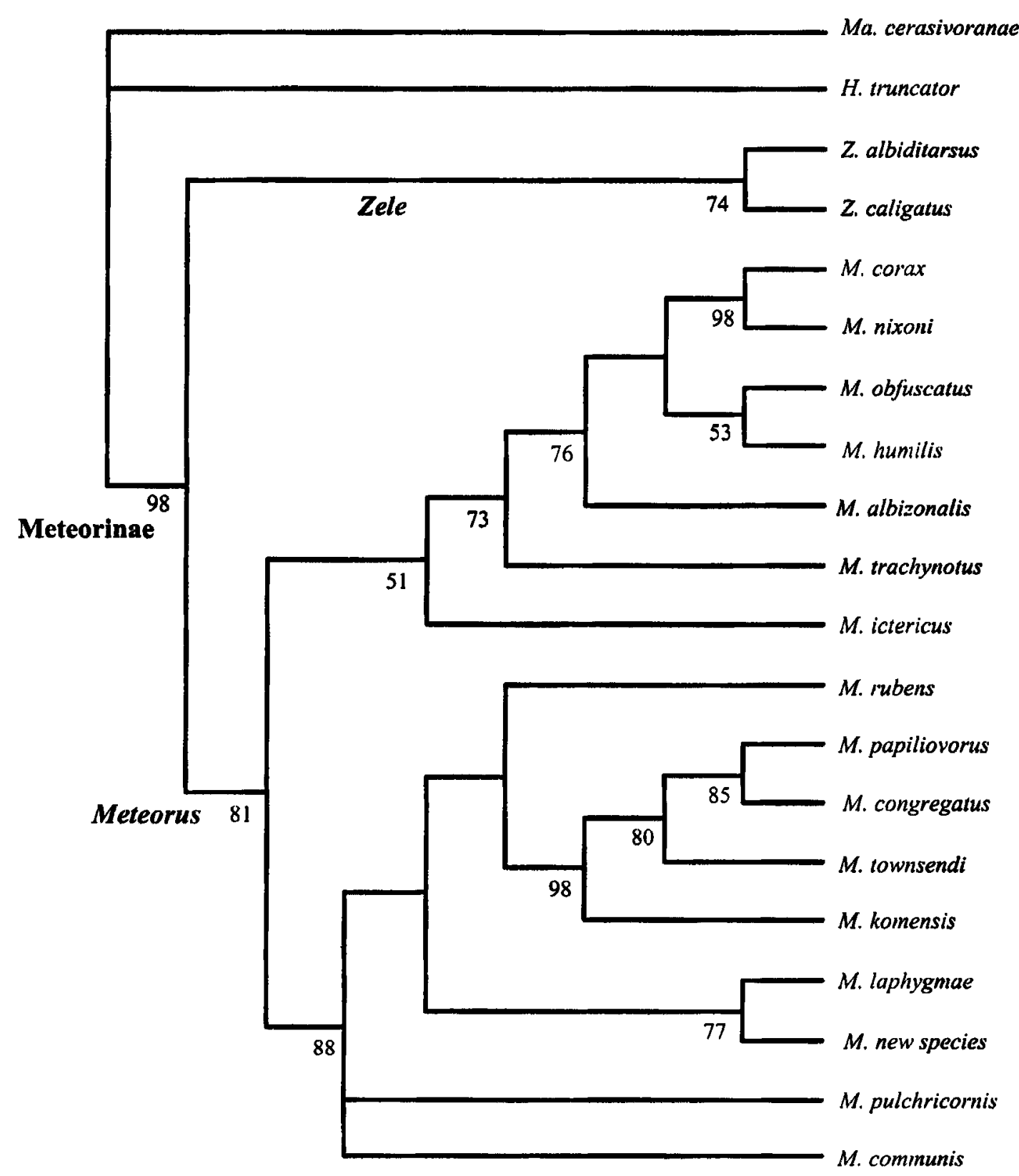

FIGURE 1. Results from the parsimony analysis of Meteorini relationships by Zitani (2003), based on morphological characters. Bootstrap values (> 50\%) are shown below the nodes.

\section{Material and methods}

\section{Studied Material}

A total of 1970 specimens were examined, of these 893 Meteorus and 87 Zele specimens were from the SMTP material. We studied all Meteorini material in the Swedish Museum of Natural History (NHRS), the Lund Zoological Museum (MZLU), the Uppsala University Department of Entomology (UUZM), the Natural History Museum in London (BMNH), the Nationaal Natuurhistorisch Museum in Leiden (RMNH) and the Zoological Institute in St. Petersburg (ZIN). Additional material, mostly type material, was borrowed from the Institut Royal des Sciences naturelles de Belgique (IRSNB), the Naturhistorisches Museum Wien (NHMW), the Museum für Naturkunde der Humboldt-Universität Berlin (ZMB), the Zoological Museum of the University of Copenhagen, Denmark (ZMUC) and from the Entomological laboratory, Faculty of Agriculture at Kyushu University Japan (KUEC). 
All material from the SMTP was originally preserved in $80 \%$ ethanol. For exact measurements, illustrations and for addition to the reference material at the NHRS, some specimens were dry-pinned, including all holotypes and some paratypes. These specimens were treated in a mixture of alcohol and xylene (60:40) for 24 hours and then glued to a piece of cardboard. Some paratypes were left in $80 \%$ ethanol for future molecular or morphological study. Most of the material, the voucher specimens and the DNA extractions are deposited in NHRS. The voucher specimen and sequence data are listed in Appendix II.

\section{Terminology and Illustrations}

The terminology used for measurements (Figs 2A-D) and descriptions of morphological characters follows Huddleston (1980) and van Achterberg (1988). The abbreviation OOL stands for Ocellar Ocular Line (Fig. 2B). Drawings were made using a Leitz microscope with camera lucida, a digital drawing board or pencil and paper, and Adobe Photoshop. The 'stacked' photographs were taken with a computer-connected Olympus DP70 digital camera and an InfinityX digital camera mounted on an Olympus SZX12 stereo microscope. Specimens for scanning electron microscopy (SEM) were dried in an ethanol gradient from $80 \%$ to $99.6 \%$, finishing with acetone. They were examined sputter-coated in a Hitachi S-4300. Additional images of most species studied here are available at Morphbank (www. morphbank.net).

\section{Species descriptions}

We have underlined the distribution records verified by label data on specimens examined by us.

\section{Morphological Phylogenetic Analysis}

For the morphological phylogenetic analysis, we started with the 20 characters described by Maeto (1989a, 1990a) and coded by him for seven species groups in the Meteorini (Table 1). We deleted a few characters, for which it was difficult to distinguish the character states, ending up with a final set of 17 characters. These characters were coded for 45 species representing the Meteorini (Table 2). The species Leiophron pallipes Curtis of the Euphorinae was used as the outgroup; it is a fairly generalized euphorine without obvious morphological specializations. Characters were coded using a Leica Z9 stereomicroscope.

The analysis of the dataset, which only includes binary characters, was performed using TNT (Goloboff et al. 2008). A heuristic search was run using 2000 random addition sequence replicates with tree bisection reconnection (TBR) branch swapping. Ten trees were held at each step during stepwise addition. Support was measured using the Group - Conflict (GC) jackknife procedure (Farris et al. 1996; Goloboff 2003) with 2000 replicates and a deletion probability for each character of $36 \%$.

\section{Molecular Analysis}

A total of 41 known species of Meteorini were studied (see Appendix II for taxa and GenBank accession numbers). The original sample of specimens from the SMTP material was chosen to cover the entire geographical range of each morphospecies identified in the material. If we suspected that a morphospecies might be a complex of cryptic species because of morphological variation or because of significant genetic variation in the initial sample of individuals, we included more specimens of the same species but from other collecting sites. We used six outgroup taxa representing five different tribes of Euphorinae (Centristini, Euphorini, Syntretini, Perilitini, Planitorini) (Boring 2010). All outgroup sequences were kindly provided by Dr A. Boring; the ingroup sequences are original to this study except the sequence of $M$. corax, which was accessed through GenBank (Appendix II).

DNA was extracted from specimens preserved in $80 \%$ ethanol or from dry-pinned specimens. In either case, only the hind leg was removed and used for DNA extraction. Two markers were sequenced for all taxa, namely the mitochondrial gene cytochrome oxidase $1(\mathrm{CO} 1 ; 658 \mathrm{bp})$ and the $\mathrm{D} 2$ loop of the nuclear 28S ribosomal sequence (28S D2; 632 bp). The CO1 primers used were LCO and HCO (Folmer et al. 1994), while 28S D2 was sequenced using the fwd and rev primers used by Campbell et al. (1993). The PCR program for CO1 had an initial 5 min 

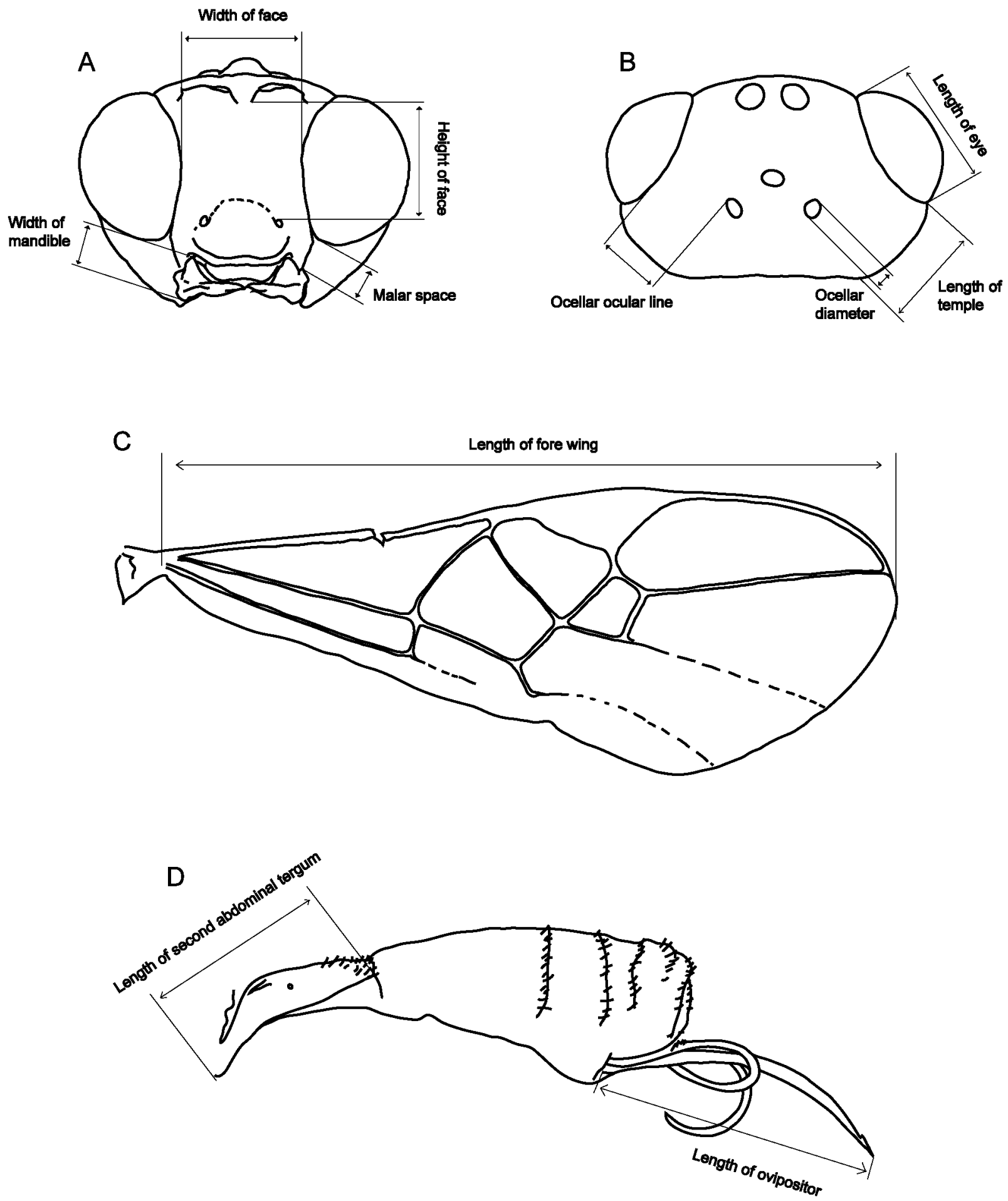

FIGURE 2. Measurements used in this paper pertaining to: A) head, anterior view; B) head, dorsal view; C) metasoma; and D) fore wing. $\mathrm{L}=$ length, $\mathrm{OOL}=$ ocellar ocular length.

denaturation at $94{ }^{\circ} \mathrm{C}$, followed by 40 cycles of $\left(94^{\circ} \mathrm{C}\right.$ for $15 \mathrm{~s}, 46{ }^{\circ} \mathrm{C}$ for $15 \mathrm{~s}$ and $72{ }^{\circ} \mathrm{C}$ for $\left.15 \mathrm{~s}\right)$, and ending with a 10 min extension period at $72{ }^{\circ} \mathrm{C}$. The PCR program for $28 \mathrm{~S}$ D2 had an initial 4 min denaturation at $95^{\circ} \mathrm{C}$, followed by 40 cycles of $\left(94^{\circ} \mathrm{C}\right.$ for $30 \mathrm{~s}, 55^{\circ} \mathrm{C}$ for $30 \mathrm{~s}$ and $72^{\circ} \mathrm{C}$ for $\left.30 \mathrm{~s}\right)$, and ending with a 10 min extension period at $72{ }^{\circ} \mathrm{C}$. PCR products were were purified using EXOFAP (EXO1 and FastAP). Gene regions were sequenced with the same primers, using the BigDyeTM Terminator ver. 3.1 Cycle Sequencing Kit (Applied Biosystems). Sequencing reactions were purified using the DyeEx 96 kit (QIAGEN) and cycle sequencing reactions were run on an ABI Prism 3100 Genetic Analyzer (Applied Biosystems). All steps were performed according to the manufacturer's instructions. For assembling and viewing the sequence data and contigs the Pregap4 and Gap4 modules of 
the Staden package (Staden et al., 1998) were used. The sequences were further processed in BioEdit (Hall, 1999) and 28S D2 was aligned using MAFFT online version (Katoh et al. 2009). CO1 was aligned by eye.

The data were partitioned into three parts: $28 \mathrm{~S}$ D2, CO1 codon positions 1+2, and CO1 codon position 3. Substitution models for the different partitions were found with MrAIC and MrModeltest (Nylander, 2004a, 2004b). GTR + I + Gamma was the optimal model for the two CO1 partitions, and GTR + Gamma for 28S D2. We used a standard non-clock tree model. The datasets for the two genes were analyzed separately and combined. In the combined analysis, all model parameters except topology and branch lengths were unlinked across partitions. Finally, we ran a combined analysis that also included the morphological characters in a separate partition.

Bayesian MCMC analyses were performed using MrBayes 3.2 (Huelsenbeck \& Ronquist, 2001; Ronquist and Huelsenbeck 2003) under default settings except as follows. The analyses were run for 5-30 million generations, until the standard deviation of split frequencies was close to or below 0.01 . The chains were sampled every 1000 generations, and $25 \%$ of the samples were discarded as burn-in. The run files are available on request.

\section{Analysis of Species Circumscription}

The generalized mixed Yule-coalecent (GMYC) model (Pons et al. 2006) was used as a complement to visual inspection to establish putative species based on the molecular data. The GMYC analysis requires an ultrametric tree. To obtain one, we used the Compound Poisson Process relaxed clock model (Huelsenbeck et al. 2000) as implemented in MrBayes 3.2, with default priors. The substitution model was the same as before. The GMYC analysis was conducted with the R package 'splits' (Species Limits by Threshold Statistics) (http://r-forge.r-project.org/ projects/splits) (Pons et al. 2006).

\section{Results}

\section{Morphological Phylogenetic Analysis}

The following discrete morphological characters were scored across all species (Table 2):

1. Size of ocelli:(0) OOL more than 2.0 the width of the lateral ocellus (Fig. 24); (1) OOL less than 2.0 the width of the lateral ocellus (Fig. 24).

2. Shape of face: (0) slightly convex or flat; (1) strongly convex (like M. micropterus Fig. 34).

3. Occipital carina: (0) complete; (1) interrupted.

4. Shape of clypeus: (0) flat (Fig. 99); (1) strongly convex (Fig. 100).

5. Convergence of eyes: (0) not convergent (Figs 82, 89, 93); (1) convergent (Fig. 86, 92, 97).

6. Mandibles: (0) stout (Fig. 98); (1) slender and twisted (Fig. 132).

7. Position of fore wing cross-vein m-cu (recurrent vein) (0) postfurcal (Fig. 30); (1) antefurcal (Fig. 62).

8. Shape of second flagellomere: (0) slender (2.5-4 times longer than wide); (1) stout (1.7-2.5 times as long as wide).

9. Structure of dorsal propodeal carinae: (0) indistinct or absent (Fig. 38); (1) distinct (Fig. 37).

10. Structure of tarsal claws: (0) simple; (1) with a distinct basal lobe.

11. Shape of pterostigma: (0) slender, vein $r$ projects apically (Figs 43, 57); (1) broad, vein $r$ projects from middle of stigma (Figs 58, 62).

12. Marginal cell of hind wing: (0) subparallel (Fig. 10b); (1) widening (Fig. 10a).

13. Dorsope: (0) absent or traces of dorsope (Figs 18, 19); (1) present (Fig. 21).

14. Ventral margins of second abdominal tergum (petiole): (0) joined mid-ventrally, or almost joining (Figs 20, 25, 26); (1) completely separate (Fig. 22).

15. Pubescence on abdominal terga 3-8: (0) one single row of setae on each tergum (Fig. 12); (1) setae evenly scattered on each tergum (Fig. 11).

16. Shape of ovipositor: (0) straight; (1) curved.

17. Length of protruding part of ovipositor: (0) short, less than twice the length of the petiolar tergum; (1) long, more than twice the length of the petiolar tergum. 
TABLE 2. Morphological character matrix.

\begin{tabular}{|c|c|c|c|c|c|c|c|c|c|c|c|c|c|c|c|c|c|}
\hline Taxa/character & 1 & 2 & 3 & 4 & 5 & 6 & 7 & 8 & 9 & 10 & 11 & 12 & 13 & 14 & 15 & 16 & 17 \\
\hline M. abdominator & 0 & 0 & 1 & 0 & 0 & 0 & 1 & 0 & 1 & 0 & 0 & 0 & 1 & 1 & 0 & 1 & 1 \\
\hline M. abscissus & 1 & 0 & 0 & 0 & 0 & 1 & 1 & 0 & 0 & 0 & 0 & 0 & 1 & 1 & 0 & 0 & 0 \\
\hline M. acerbiavorus & 1 & 0 & 0 & 1 & 0 & 1 & 1 & 1 & 0 & 0 & 0 & 0 & 0 & 0 & 0 & 0 & 0 \\
\hline M. affinis & 1 & 0 & 0 & 0 & 1 & 0 & 1 & 0 & 0 & 1 & 0 & 0 & 1 & 1 & 0 & 0 & 1 \\
\hline M. artocercus & 0 & 0 & 0 & 1 & 1 & 1 & 1 & 0 & 0 & 1 & 0 & 0 & 0 & 0 & 0 & 0 & 1 \\
\hline M. brevicauda & 0 & 0 & 0 & 0 & 0 & 0 & 1 & 1 & 1 & 0 & 1 & 0 & 1 & 1 & 0 & 0 & 0 \\
\hline M. cespitator & 0 & 0 & 0 & 0 & 0 & 0 & 1 & 1 & 0 & 0 & 0 & 0 & 1 & 1 & 0 & 0 & 1 \\
\hline M. cinctellus & 0 & 0 & 0 & 1 & 1 & 1 & 0 & 0 & 0 & 1 & 0 & 0 & 0 & 0 & 0 & 0 & 1 \\
\hline M. cis & 0 & 0 & 0 & 0 & 0 & 0 & 1 & 1 & 1 & 0 & 0 & 0 & 1 & 1 & 0 & 0 & 1 \\
\hline M. colon & 0 & 0 & 0 & 1 & 1 & 1 & 0 & 0 & 0 & 1 & 0 & 0 & 0 & 0 & 0 & 0 & 0 \\
\hline M. consimilis & 1 & 0 & 0 & 1 & 0 & 1 & 1 & 1 & 0 & 1 & 0 & 0 & 1 & 1 & 0 & 1 & 0 \\
\hline M. corax & 0 & 0 & 0 & 0 & 0 & 0 & 1 & 0 & 0 & 1 & 0 & 0 & 1 & 1 & 0 & 0 & 1 \\
\hline M. densipilosus & 0 & 0 & 0 & 0 & 0 & 0 & 1 & 0 & 0 & 0 & 1 & 0 & 1 & 1 & 0 & 0 & 1 \\
\hline M. eadyi & 0 & 0 & 0 & 0 & 0 & 0 & 1 & 0 & 1 & 0 & 1 & 0 & 0 & 0 & 0 & 1 & 0 \\
\hline M. eklundi & 0 & 0 & 0 & 0 & 1 & 0 & 1 & 1 & 0 & 0 & 1 & 0 & 1 & 1 & 0 & 1 & 0 \\
\hline M. filator & 0 & 0 & 0 & 0 & 1 & 0 & 1 & 0 & 1 & 0 & 1 & 0 & 0 & 0 & 0 & 0 & 1 \\
\hline M. hirsutipes & 0 & 0 & 0 & 0 & 0 & 0 & 1 & 0 & 1 & 0 & 1 & 0 & 1 & 1 & 0 & 0 & 1 \\
\hline M. ictericus & 1 & 0 & 0 & 1 & 0 & 1 & 1 & 0 & 0 & 1 & 1 & 0 & 1 & 1 & 0 & 0 & 0 \\
\hline M. jaculator & 0 & 0 & 0 & 0 & 0 & 0 & 1 & 0 & 1 & 1 & 1 & 0 & 1 & 1 & 0 & 0 & 1 \\
\hline M. lionotus & 1 & 0 & 0 & 1 & 0 & 1 & 1 & 0 & 0 & 1 & 0 & 0 & 0 & 0 & 0 & 0 & 0 \\
\hline M. longicaudis & 0 & 0 & 0 & 0 & 0 & 0 & 0 & 0 & 0 & 0 & 1 & 0 & 1 & 1 & 0 & 0 & 1 \\
\hline M. longipilosus & 0 & 0 & 0 & 1 & 0 & 0 & 1 & 0 & 1 & 1 & 0 & 0 & 1 & 1 & 0 & 1 & 1 \\
\hline M. melanostictus & 1 & 0 & 0 & 0 & 0 & 1 & 0 & 0 & 0 & 1 & 0 & 0 & 1 & 1 & 0 & 0 & 0 \\
\hline M. mellifacialis & 0 & 0 & 0 & 0 & 0 & 0 & 1 & 1 & 1 & 1 & 1 & 0 & 1 & 1 & 0 & 0 & 1 \\
\hline M. micropterus & 0 & 1 & 0 & 0 & 0 & 0 & 1 & 1 & 0 & 0 & 0 & 0 & 0 & 0 & 0 & 0 & 1 \\
\hline M. obfuscatus & 0 & 0 & 0 & 0 & 0 & 0 & 1 & 1 & 1 & 0 & 1 & 0 & 1 & 1 & 0 & 0 & 1 \\
\hline M. obsoletus & 1 & 0 & 0 & 0 & 1 & 1 & 0 & 0 & 0 & 1 & 0 & 0 & 0 & 0 & 0 & 0 & 1 \\
\hline M. oculatus & 0 & 0 & 0 & 0 & 1 & 0 & 1 & 0 & 1 & 1 & 1 & 0 & 1 & 1 & 0 & 0 & 1 \\
\hline M.pendulus & 1 & 0 & 0 & 1 & 1 & 1 & 0 & 0 & 0 & 1 & 0 & 0 & 1 & 1 & 0 & 0 & 0 \\
\hline M. pulchricornis & 1 & 0 & 0 & 1 & 1 & 1 & 1 & 0 & 0 & 1 & 0 & 0 & 0 & 0 & 0 & 0 & 0 \\
\hline M. punctifrons & 0 & 0 & 1 & 0 & 0 & 0 & $?$ & 0 & 0 & 0 & 1 & 0 & 1 & 1 & 0 & 1 & 0 \\
\hline M. rubens & 1 & 0 & 0 & 1 & 0 & 1 & 1 & 0 & 0 & 0 & 0 & 0 & 0 & 0 & 0 & 0 & 0 \\
\hline M. ruficeps & 1 & 0 & 0 & 1 & 1 & 1 & 1 & 0 & 1 & 1 & 0 & 0 & 1 & 1 & 0 & 0 & 1 \\
\hline M. salicorniae & 0 & 1 & 0 & 0 & 0 & 0 & 1 & 1 & 0 & 0 & 1 & 0 & 1 & 1 & 0 & 0 & 1 \\
\hline M. sibyllae & 0 & 0 & 0 & 0 & 0 & 0 & 1 & 0 & 1 & 0 & 1 & 0 & 1 & 1 & 0 & 0 & 1 \\
\hline M. stenomastax & 0 & 0 & 0 & 1 & 1 & 1 & 0 & 0 & 0 & 1 & 0 & 0 & 0 & 0 & 0 & 0 & 1 \\
\hline M. subtilisulcus & 0 & 0 & 0 & 0 & 0 & 0 & 1 & 0 & 1 & 0 & 1 & 0 & 1 & 1 & 0 & 0 & 0 \\
\hline M. sulcatus & 1 & 0 & $?$ & 0 & 0 & 0 & $?$ & 0 & 0 & 0 & 1 & 0 & 1 & 1 & 0 & 0 & 1 \\
\hline M. tabidus & 1 & 0 & 0 & 1 & 1 & 1 & 1 & 0 & 0 & 1 & 0 & 0 & 0 & 1 & 0 & 0 & 1 \\
\hline M.tenellus & 0 & 0 & 0 & 1 & 1 & 1 & 1 & 0 & 0 & 1 & 0 & 0 & 0 & 0 & 0 & 0 & 1 \\
\hline M. versicolor & 1 & 0 & 0 & 1 & 0 & 1 & 0 & 0 & 0 & 1 & 0 & 0 & 0 & 0 & 0 & 0 & 0 \\
\hline M. vexator & 0 & 0 & 0 & 0 & 1 & 0 & 1 & 0 & 0 & 1 & 1 & 0 & 1 & 1 & 0 & 0 & 1 \\
\hline Z. albiditarsus & 1 & 0 & 0 & 0 & 0 & 1 & 1 & 0 & 1 & 1 & 0 & 1 & 1 & 1 & 1 & 1 & 0 \\
\hline Z. deceptor & 1 & 0 & 0 & 0 & 0 & 1 & 1 & 0 & 1 & 1 & 0 & 1 & 1 & 1 & 1 & 1 & 1 \\
\hline Z. chlorophthalmus & 1 & 0 & 0 & 0 & 0 & 1 & 1 & 0 & 1 & 1 & 0 & 1 & 1 & 1 & 1 & 1 & 1 \\
\hline Z. caligatus & 1 & 0 & 1 & 0 & 0 & 1 & 1 & 0 & 1 & 1 & 0 & 1 & 1 & 1 & 1 & 1 & 0 \\
\hline Leiophron pallipes & 0 & 0 & 1 & 0 & 0 & 1 & $?$ & 1 & 0 & 0 & 1 & 0 & 1 & 1 & 1 & $?$ & $?$ \\
\hline
\end{tabular}

Parsimony analysis of the morphological data resulted in 1310 trees of equal length, the strict consensus of which was largely unresolved (Fig. 3). Jackknife anlysis indicated fairly strong support (GC jackknife proportion $81 \%$ ) for the monophylyt of Zele but no support above $50 \%$ for any other group in the tree. 


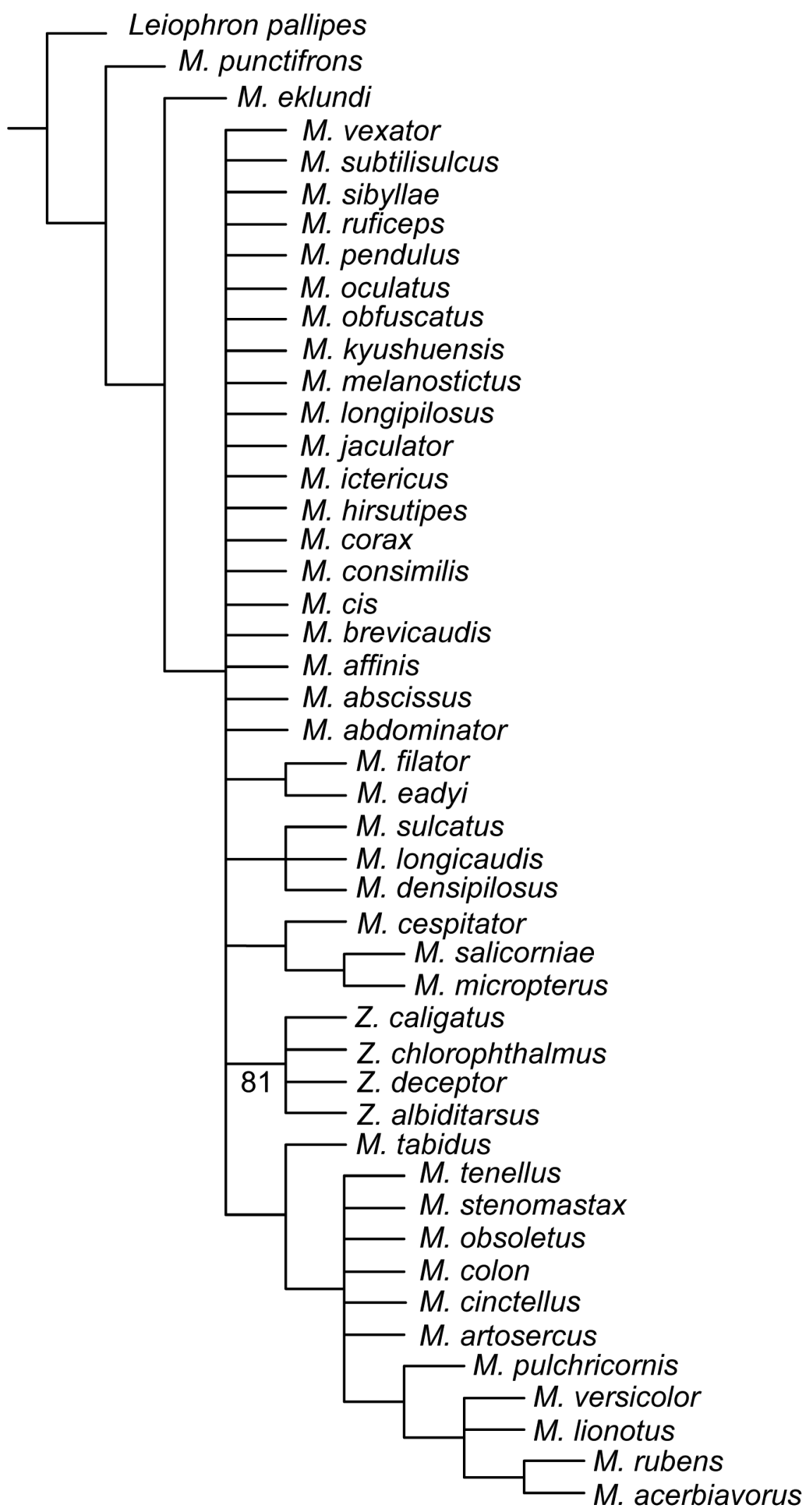

FIGURE 3. Results of parsimony analysis of the morphological matrix. GC jackknife support value (in percentage units) shown below the corresponding branch. 


\section{Molecular and Combined Phylogenetic Analyses}

The Bayesian MCMC analysis of the combined molecular data (Fig. 4) shows that the Meteorini fall into four wellsupported clades. The first clade (Clade I, Fig. 4) includes M. ictericus and M. ruficeps; they form the sister group of the remaining Meteorini. The next clade (Clade II, Fig. 4) includes 13 species, all of which are parasitoids on Lepidoptera larvae. The members of Clade II are all slender and long-legged species, their ocelli tend to be large, most species are bicoloured, and most lack a dorsope except M. abscissus, M. limbatus and M. pendulus, which have a large, distinct dorsope, and M. rubens and M. pulchrichornis, which have a small and indistinct dorsope. Meteorus micropterus (Clade IIC) appears to be the sister lineage of the rest of Clade II, which falls into two strongly supported subclades of about equal size (Clade IIA and Clade IIB; Fig. 4).

Members of the third clade (Clade III, Fig. 4) parasitize both Lepidoptera and Coleoptera. They all have a distinct dorsope and small ocelli. Relationships among members of this clade are poorly resolved. The fourth clade (Clade IV, Fig. 7) consists entirely of species belonging to Zele. Within Zele, the two species Z. albiditarsus and Z. deceptor, previously considered conspecific, are clearly shown to be distinct species. The analysis suggests that Clade IV (Zele) is the sister of Clade III, but only with moderate support (79\% Posterior Probability (PP)).

The CO1 sequences on their own nicely separate species but higher relationships are poorly resolved for the most part (Fig. 5). However, Clade I and Clade IV are both strongly supported (100\% PP). The core of Clade II (IIA + IIB, that is, all species except M. micropterus) is also present (100\% PP), while Clade III falls into several different clades appearing in a large basal polytomy in the Meteorini. The monophyly of the Meteorini receives modest support (75\% PP).

The $28 \mathrm{~S}$ topology (Fig 6) is poorly resolved at the species level but provides better support for the basal clades in the Meteorini. The monophyly of Meteorini receives moderate support (60\% PP), while Clade I, Clade II and Clade IV all are strongly supported as monophyletic (98-100\% PP). Clade III, on the other hand, falls into a number of separate lineages that appear basally in the group formed by (Clade II + III + IV). The monophyly of (Clade $\mathrm{II}+\mathrm{III}+\mathrm{IV})$ receives moderate support $(60 \% \mathrm{PP})$.

When the molecular evidence is combined with morphological data for a slightly expanded set of species (45 compared with 39), the result is a highly polytomous topology (Fig. 7). The lack of resolution is entirely due to the difficulty of placing the six additional species based solely on their morphology; when these species are removed, the consensus from the analysis of the combined morphological and molecular data is very similar to that from the combined molecular analysis (cf. Fig. 4).

\section{Species Circumscription}

All species recognized based on morphology appeared as reciprocally monophyletic in the combined molecular analysis (Fig. 4). The molecular data also indicated the presence of 12 additional, cryptic species in eight morphospecies: M. ruficeps, M. tenellus, M. rubens, M. affinis, M. pendulus, M. cis, M. obfuscatus, and M. filator. These cryptic species were separated from their closest relatives by considerable genetic distances (well over $5 \%$ ) in both the $\mathrm{CO} 1$ (Fig. 5) and combined analyses (Fig. 4). Five of these 12 cryptic species also have genetically distinct $28 \mathrm{~S}$ sequences; the exceptions are $M$. filator, in which the 28S sequences are identical in the two cryptic species, and M. tenellus, in which the $28 \mathrm{~S}$ sequences of the three cryptic species are part of an ancestral set of gene copies shared with M. stenomastax, M. artocercus and M. cinctellus (Fig. 6).

Despite careful study, we were not able to find consistent morphological differences separating the 12 cryptic species from their closest relatives. Some species complexes are morphologically homogeneous (M. pendulus, M. filator, M. cis), while others show considerable variation (M. ruficeps, M. tenellus, M. rubens, M. affinis) without the cryptic species being distinguishable. Many of the cryptic species have partially overlapping geographic ranges (those belonging to $M$. tenellus, $M$. rubens, $M$. pendulus, $M$. filator, and $M$. cis complexes), while others are separated geographically as far as can be judged from current data (those belonging to the M. ruficeps, M. obfuscatus and $M$. affinis complexes). We do not have biological data for the cryptic species, so it is impossible to determine whether there are differences among them in host preferences or other life-history traits. 


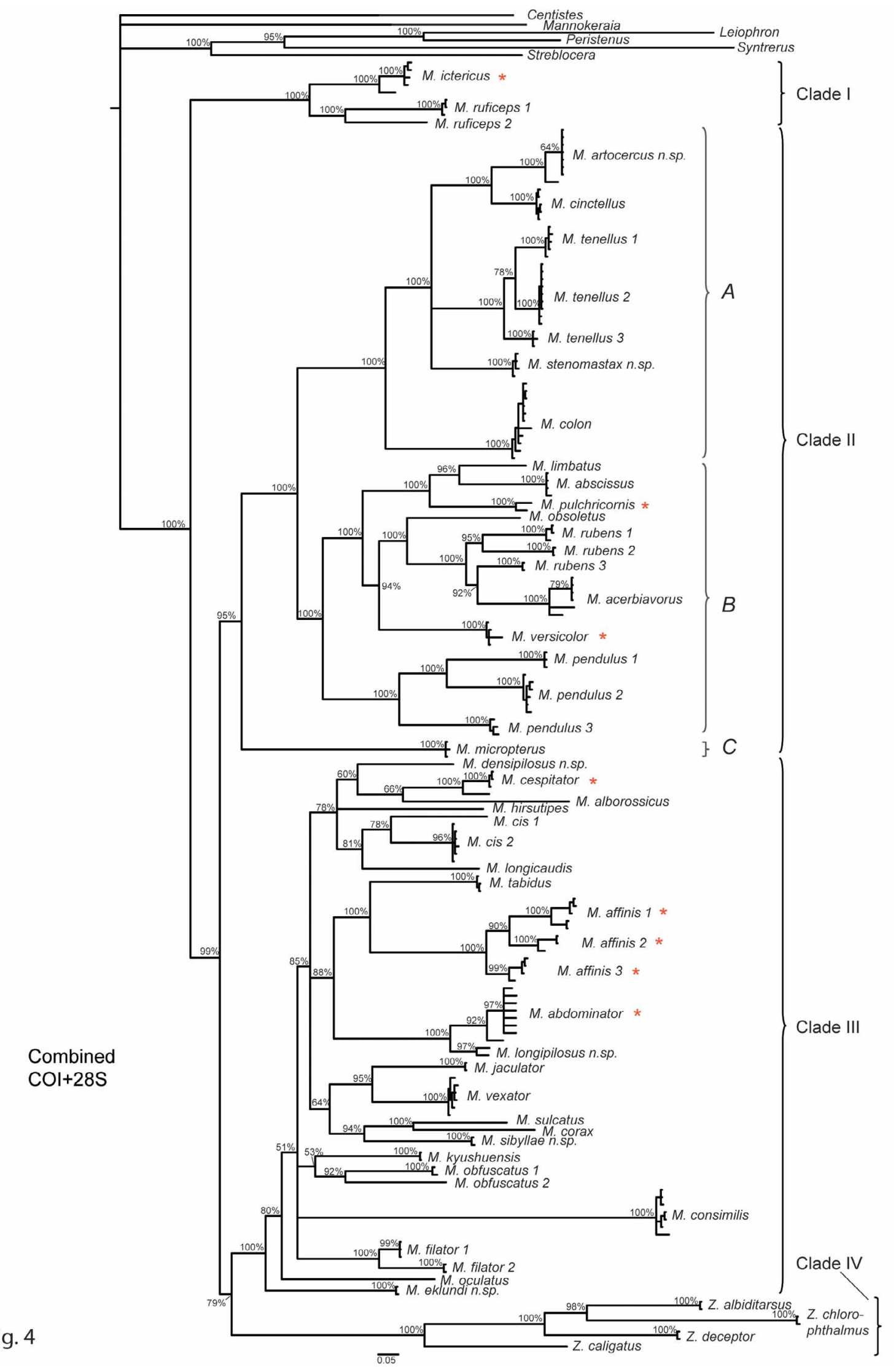

FIGURE 4. Results from Bayesian phylogenetic analysis of the combined molecular data (CO1 and 28S D2). Numbers on branches are posterior probabilities (PP; in percent); branches with PP below $50 \%$ are collapsed. PP values are not given for branches inside the terminal clusters corresponding to species. Putative major clades of the Meteorinae referred to in the text are indicated to the right. The scale bar represents nucleotide substitutions per site, and all Bayesian trees are rooted between the genera Manokeria and Leiophron. 


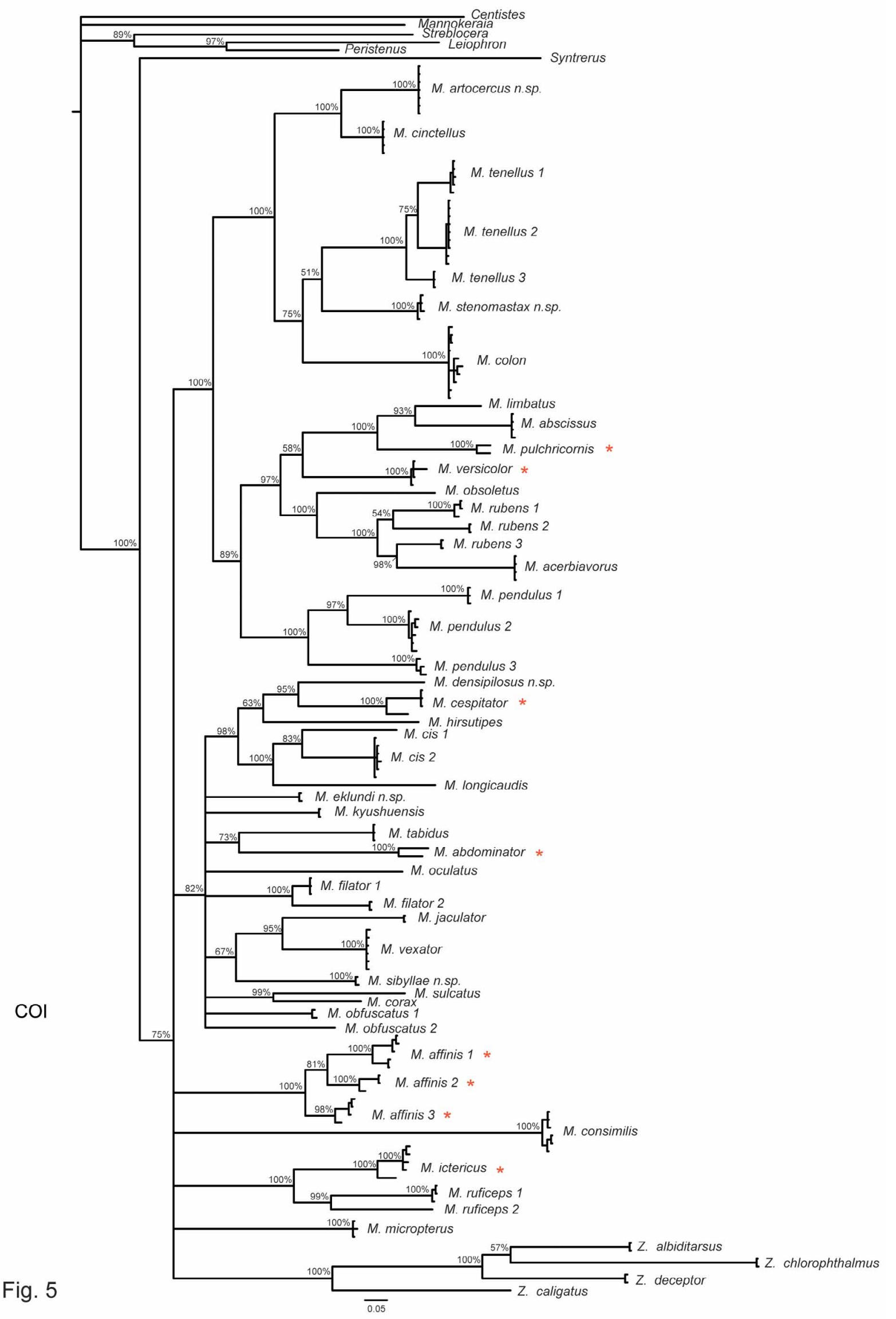

FIGURE 5. Results from Bayesian phylogenetic analysis of the $\mathrm{CO} 1$ data. The scale bar represents nucleotide substitutions per site. For additional explanation, see Fig. 4. 


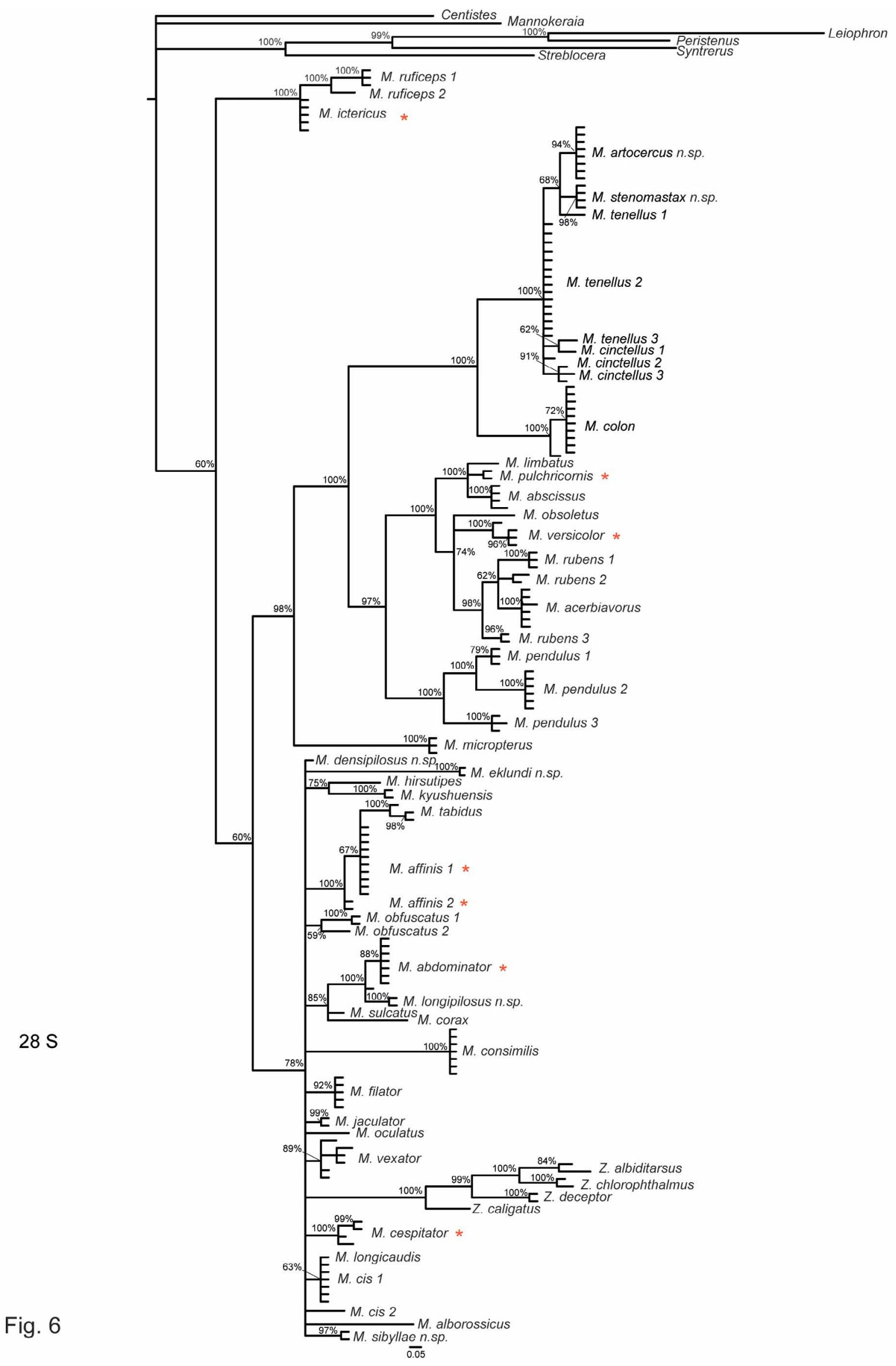

FIGURE 6. Results from Bayesian phylogenetic analysis of the 28S D2 data. The scale bar represents nucleotide substitutions per site. For additional explanation, see Fig. 4. 


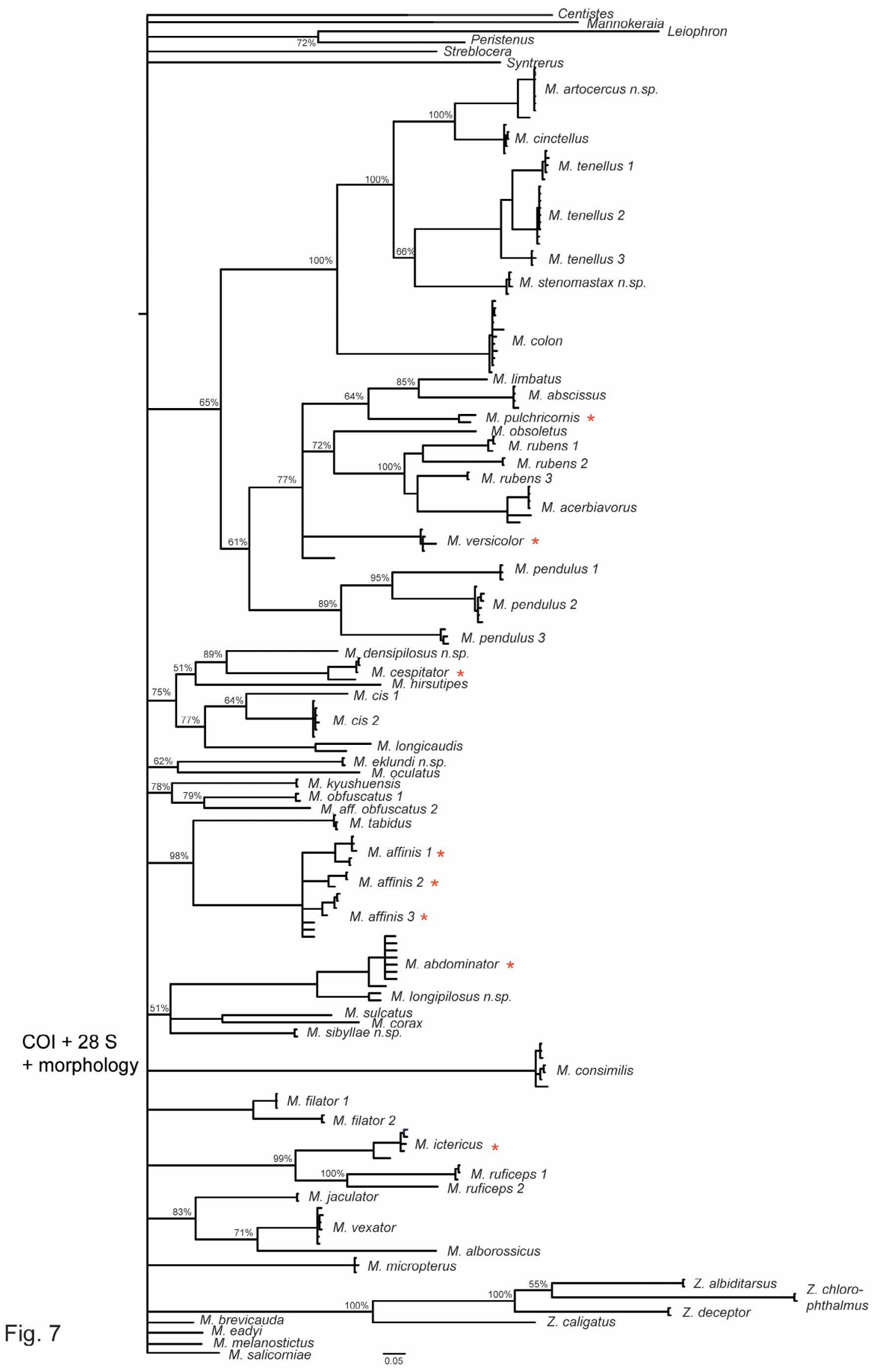

FIGURE 7. Results from Bayesian phylogenetic analysis of the combined CO1, 28S D2 and morphology data. The scale bar represents nucleotide substitutions per site. For additional explanation, see Fig. 4. 


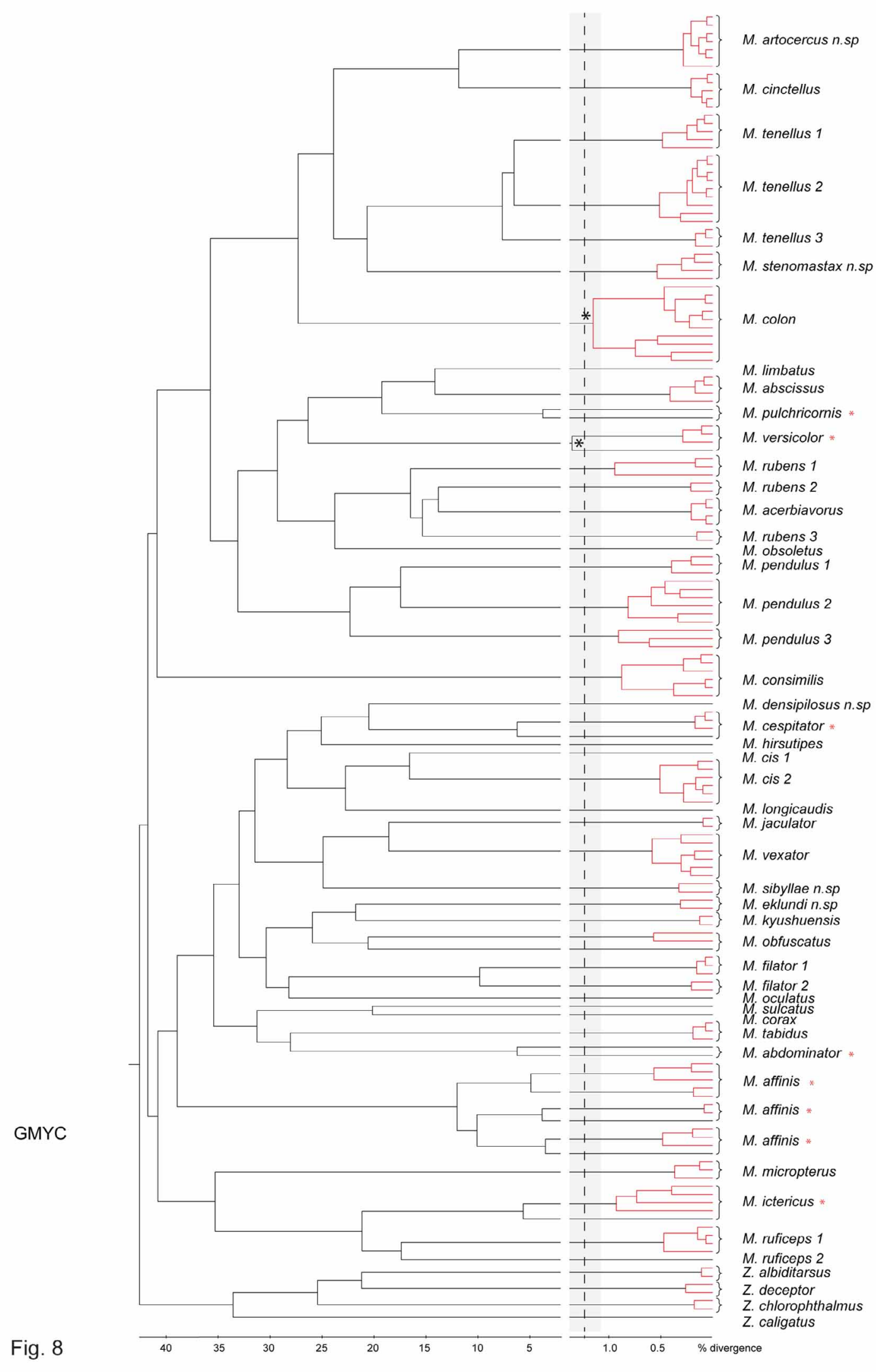

FIGURE 8. Results from the GMYC analysis. The grey bar represents the confidence interval for the maximum likelihood estimate of the change point between the Yule and coalescent branching models $( \pm 2$ log likelihood units from the maximum likelihood value). The terminal part of the tree is magnified 10 times for better visibility of the suggested species boundaries. Contiguous red clades are indicated to be conspecific according to the maximum likelihood estimate. The scale bar represents nucleotide substitutions per site. 
The GMYC analysis of CO1 data confirmed the distinctness of the 12 cryptic species but indicated the presence of 8 additional species (Fig. 8). In general, the additional GMYC lineages represented single individuals that had unique CO1 sequences but shared 28S sequences with other specimens (Figs 4-6). In M. affinis, however, the five extra GMYC lineages represented significant $\mathrm{CO} 1$ variation across specimens, suggesting the presence of at least three major groups (Fig. 5). This was not reflected by the $28 \mathrm{~S}$ sequences, which were mostly identical; two aberrant basal sequences were found in specimens that were deeply nested in the CO1 tree. The GMYC results were the same with or without identical CO1 sequences (haplotypes) removed.

\section{Evolution of Host Preferences}

When host preferences (Coleoptera or Lepidoptera) are mapped onto the tree from the combined molecular analysis, a clear pattern emerges (Fig. 9). Clades I, II, and IV are all completely specialized on lepidopteran hosts, which clearly suggests that this represents the ancestral host state of the Meteorini. Members of Clade III attack both coleopteran and lepidopteran hosts. The coleopteran parasitoids do not seem to form a monophyletic subgroup; instead, it appears that members of Clade III have shifted several times between lepidopteran and coleopteran hosts. However, support values are low for many groups, and host preferences are also unknown for some of the key taxa, making it difficult to draw robust conclusions concerning host shifts in this clade.

\section{Discussion}

\section{Phylogeny}

Our results clearly indicate that the Meteorini fall into four distinct clades, here named Clade I to Clade IV, with Clade I being the sister group of the others (Fig. 4). The results are weaker when it comes to relationships among Clades II, III, and IV, but they do suggest that Clade II may be the sister to (Clade III + Clade IV). Since Clade IV corresponds to Zele, our results indicate that Zele is deeply nested within Meteorus.

Our study thus conflicts with the results of Zitani's (2003) phylogenetic analyses based on 39 morphological characters, indicating that Meteorus and Zele are sister groups (Fig. 1). One possible reason for the discrepancy might be that Zitani's selection of species is strongly biased in favor of lepidopteran parasitoids, while many of the closest relatives of Zele in Meteorus are coleopteran parasitoids according to our analysis (Figs 4,9). Our analysis (Figs 3,7) also indicates that morphological characters are relatively uninformative about phylogeny in Meteorini, although it should be pointed out that Zitani used a larger set of morphological characters than that used by us, including a number of larval and pupal characters that we were unable to score for the species analyzed here.

Our study generally supports Maeto's (1990a) hypothesis of Zele being nested within Meteorus but disagrees in the details. Maeto specifically suggested that Zele forms a monophyletic group with his ictericus, rubens and pulchricornis groups (Table 1) of Meteorus. According to our results, however, the ictericus, rubens and pulchricornis groups of Maeto are not monophyletic, nor do they include the closest relatives of Zele.

The species groups formed by Maeto (1990a) were based on morphology and mostly included Japanese species. They partly fit our phylogenetic results and partly conflict with them (Table 3). Maeto's ictericus group (Table 1) includes both M. ictericus and M. ruficeps, which form Clade I in our analysis (Fig. 4), and a number of other species that form scattered lineages in Clade III. The core of the ictericus group (our Clade I) is characterized by: (1) deep and large laterope and dorsope; (2) long antennae; (3) ovipositor longer than second abdominal tergum (petiole); (4) tarsal claws distinctly angulated and with a sharp basal tooth. Of the species listed by Maeto in the ictericus group, M. affinis and M. tabidus belong to Clade III (Fig. 4). We have not had access to material of the other species in Maeto's ictericus group (Table 1) and cannot comment on their position.

Our clade II falls into three separate lineages. The first one (Clade IIA) consists of M. artocercus, M. colon, M. cinctellus, M. stenomastax, and M. tenellus. It largely corresponds to Maeto's colon subgroup of the pulchricornis group. This group is characterized by: (1) no dorsope; (2) face narrow; (3) mandibles strongly twisted; (4) median tubercle on frons; (5) ovipositor long and slender. The first author examined type material of M. melanostictus, and concluded that this species is more closely related to Clade III than to the other species in Maeto's colon subgroup (Table 3). We have not seen material of M. watanabei, the last species included by Maeto in the colon subgroup. 


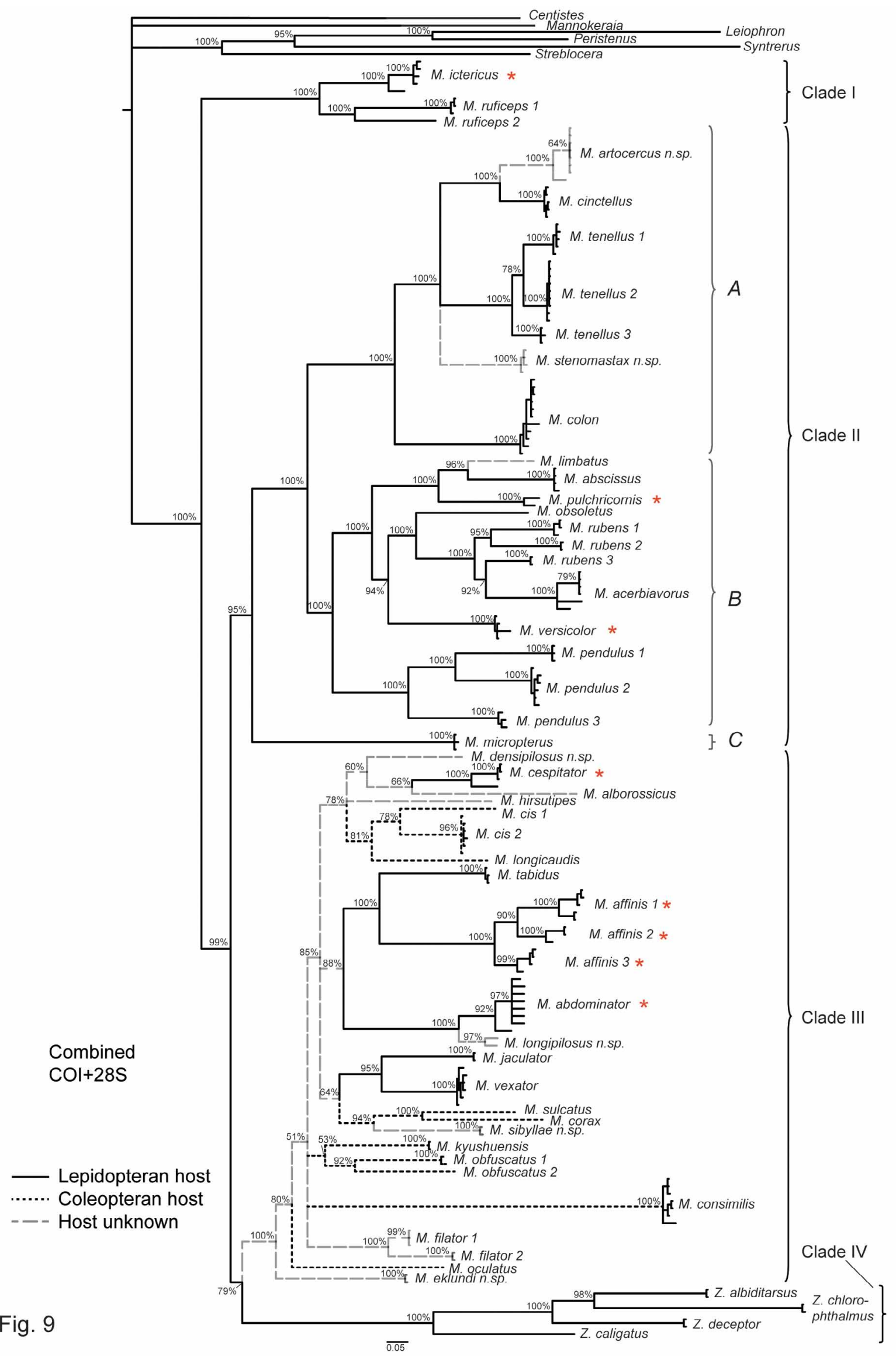

FIGURE 9. Reconstruction of host preferences based on Fitch parsimony but marking ancestral branches as ambiguous when there is a complete lack of data on all descendants (most of these branches are reconstructed as having a lepidopteran host by Fitch parsimony). 
TABLE 3. List of species studied in this paper, indicating the likely placement of each species in the major clades of the Meteorini that emerged in our phylogenetic analysis (Fig. 4). Species preceded by an asterisk were studied after the phylogenetic analysis had been completed; their placement is based on our interpretation of the available morphological evidence. The placement of all other species is based on the molecular phylogenetic analysis (Fig. 4).

\begin{tabular}{|c|c|c|c|}
\hline Clade & Taxon & Distribution & Host family \\
\hline \multirow[t]{2}{*}{ Clade I } & M. ictericus (Nees von Esenbeck, 1811) & $\begin{array}{l}\text { Western Palearctic, } \\
\text { Eastern Palearctic, } \\
\text { Nearctic, Oriental, } \\
\text { Australasia }\end{array}$ & Geometridae, Noctuidae, Tortricidae (Lep.) \\
\hline & M.ruficeps (Nees von Esenbeck, 1811) & Western Palearctic & Geometridae, Tortricidae (Lep.) \\
\hline \multirow[t]{5}{*}{ Clade IIA } & M. artocercus Stigenberg n.sp. & Western Palearctic & Arctiidae \\
\hline & M. cinctellus Spinola, 1808 & $\begin{array}{l}\text { Western Palearctic, } \\
\text { Eastern Palearctic, } \\
\text { Australasia }\end{array}$ & Tortricidae (Lep.) \\
\hline & M. colon (Haliday, 1835) & $\begin{array}{l}\text { Western Palearctic, } \\
\text { Eastern Palearctic }\end{array}$ & Noctuidae (Lep.) \\
\hline & M.tenellus Marshall, 1887 & Western Palearctic & Tortricidae (Lep.) \\
\hline & M. stenomastax Stigenberg n.sp. & Western Palearctic & \\
\hline \multirow[t]{16}{*}{ Clade IIB } & M.abscissus Thomson, 1895 & $\begin{array}{l}\text { Western Palearctic, } \\
\text { Eastern Palearctic }\end{array}$ & Arctiidae, Geometridae, Noctuidae (Lep.) \\
\hline & M. acerbiavorus Belokobylskij et al. 2011 & Western Palearctic & Arctiidae (Lep.) \\
\hline & *M. heliophilus (Fischer, 1970) & $\begin{array}{l}\text { Western Palearctic, } \\
\text { Eastern Palearctic }\end{array}$ & $\begin{array}{l}\text { Arctiidae, Geometridae, Pyralidae, } \\
\text { Tortricidae (Lep.) }\end{array}$ \\
\hline & *M. kurokoi Maeto, 1989 & Eastern Palearctic & Unknown \\
\hline & *M. insulicola Maeto, 1989 & Eastern Palearctic & Unknown \\
\hline & M.limbatus Maeto, 1988 & $\begin{array}{l}\text { Western Palearctic, } \\
\text { Eastern Palearctic }\end{array}$ & Unknown \\
\hline & *M. lionotus Thomson, 1895 & Western Palearctic & Geometridae (Lep.) \\
\hline & *M. nodai Maeto, 1988 & Eastern Palearctic & Unknown \\
\hline & M.obsoletus (Wesmael, 1835) & $\begin{array}{l}\text { Western Palearctic, } \\
\text { Eastern Palearctic }\end{array}$ & mostly Tortricidae (Lep) \\
\hline & M.pendulus (Müller, 1776) & $\begin{array}{l}\text { Western Palearctic, } \\
\text { Eastern Palearctic }\end{array}$ & $\begin{array}{l}\text { Geometridae, Lasiocampidae, Lycaenidae, } \\
\text { Lymantriidae, Noctuidae, Tortricidae (Lep.) }\end{array}$ \\
\hline & *M. politutele Shenefelt, 1969 & Western Palearctic & Unknown \\
\hline & M.pulchricornis (Wesmael, 1835) & $\begin{array}{l}\text { Western Palearctic, } \\
\text { Eastern Palearctic }\end{array}$ & 18 families of Lepidoptera, mostly Noctuidae \\
\hline & M.rubens (Nees, 1811) & Palearctic, Nearctic & Mostly Noctuidae (Lep.) \\
\hline & *M. rufus (DeGeer, 1778) & Western Palearctic & $\begin{array}{l}7 \text { families of Lepidoptera, especially } \\
\text { Zygaenidae }\end{array}$ \\
\hline & *M.takenoi Maeto, 1989 & Eastern Palearctic & Unknown \\
\hline & M. versicolor (Wesmael, 1835) & $\begin{array}{l}\text { Western Palearctic, } \\
\text { Eastern Palearctic }\end{array}$ & $\begin{array}{l}\text { mostly Geometridae, Lasiocampidae, } \\
\text { Lymantriidae, Noctuidae (Lep.) }\end{array}$ \\
\hline Clade IIC & M. micropterus (Haliday, 1835) & $\begin{array}{l}\text { Western Palearctic, } \\
\text { Eastern Palearctic }\end{array}$ & Hepialidae (Lep.) \\
\hline \multirow[t]{3}{*}{ Clade III } & M. abdominator (Nees, 1811) & $\begin{array}{l}\text { Western Palearctic, } \\
\text { Eastern Palearctic }\end{array}$ & mostly Geometridae (Lep.) \\
\hline & M. affinis (Wesmael, 1835) & $\begin{array}{l}\text { Western Palearctic, } \\
\text { Eastern Palearctic }\end{array}$ & mostly Psychidae (Lep.) \\
\hline & M. alborossicus Lobodenko, 2000 & Western Palearctic & Unknown \\
\hline
\end{tabular}

...... continued on the next page 
TABLE 3 (continued)

\begin{tabular}{|c|c|c|c|}
\hline Clade & Taxon & Distribution & Host family \\
\hline & *M. angiclypealis van Achterberg, 2001 & Western Palearctic & Unknown \\
\hline & \multicolumn{3}{|l|}{ *M. angustatus Maeto,1988 } \\
\hline & *M. breviantennatus Tobias, 1986 & $\begin{array}{l}\text { Western Palearctic, } \\
\text { Eastern Palearctic }\end{array}$ & Curculionidae (Col.) \\
\hline & *M. brevicauda Thomson, 1895 & $\begin{array}{l}\text { Western Palearctic, } \\
\text { Eastern Palearctic }\end{array}$ & Chrysomelidae (Col.) \\
\hline & M. cespitator (Thunberg, 1824) & $\begin{array}{l}\text { Western Palearctic, } \\
\text { Eastern Palearctic, } \\
\text { Oceania }\end{array}$ & Tineidae (Lep.) \\
\hline & M. cis (Bouché, 1834) & $\begin{array}{l}\text { Western Palearctic, } \\
\text { Eastern Palearctic }\end{array}$ & Curculionidae (Col.) \\
\hline & M. consimilis (Nees, 1834) & Western Palearctic & Curculionidae (Col.) \\
\hline & M. corax Marshall, 1898 & $\begin{array}{l}\text { Western Palearctic, } \\
\text { Eastern Palearctic }\end{array}$ & mostly Cerambycidae (Col.) \\
\hline & *M. curvus Maeto, 1988 & Eastern Palearctic & Unknown \\
\hline & M. densipilosus Stigenberg n.sp. & Western Palearctic & Unknown \\
\hline & *M. eadyi Huddleston, 1980 & $\begin{array}{l}\text { Western Palearctic, } \\
\text { Mongolia }\end{array}$ & Unknown \\
\hline & M. eklundi Stigenberg n.sp. & Western Palearctic & Unknown \\
\hline & *M. endoclytae Maeto, 1990 & Eastern Palearctic & Hepialidae (Lep.) \\
\hline & M. filator (Haliday, 1835) & Western Palearctic & Unknown \\
\hline & *M. gotoi Maeto, 1988 & Eastern Palearctic & Unknown \\
\hline & M. hirsutipes Huddleston, 1980 & $\begin{array}{l}\text { Western Palearctic, } \\
\text { Eastern Palearctic }\end{array}$ & Unknown \\
\hline & *M. ipidivorus Tobias, 1986 & Western Palearctic & Curculionidae (Col.) \\
\hline & M. jaculator (Haliday, 1835) & Western Palearctic & Gelechiidae, Psychidae, Tineidae (Lep.) \\
\hline & *M. jezoensis Maeto, 1988 & Eastern Palearctic & Unknown \\
\hline & M. kyushuensis Maeto, 1988 & $\begin{array}{l}\text { Western Palearctic, } \\
\text { Eastern Palearctic }\end{array}$ & Erotylidae (Col.) \\
\hline & M. longicaudis (Ratzeburg, 1848) & Western Palearctic & Tenebrionidae (Col.) \\
\hline & M. longipilosus Stigenberg n.sp. & Western Palearctic & Unknown \\
\hline & *M. melanostictus Capron, 1887 & $\begin{array}{l}\text { Western Palearctic, } \\
\text { Eastern Palearctic }\end{array}$ & mostly Geometridae, Tortricidae \\
\hline & *M. micropilosus Tobias, 1986 & Western Palearctic & Unknown \\
\hline & *M. nadeshdae Lobodenko, 2000 & Eastern Palearctic & Unknown \\
\hline & *M. nixoni Huddleston, 1980 & $\begin{array}{l}\text { Western Palearctic, } \\
\text { Eastern Palearctic }\end{array}$ & Unknown \\
\hline & M.obfuscatus (Nees, 1811) & $\begin{array}{l}\text { Western Palearctic, } \\
\text { Eastern Palearctic }\end{array}$ & $\begin{array}{l}\text { Cerambycidae, Erotylidae, Melandryidae, } \\
\text { Tenebrionidae (Col.) }\end{array}$ \\
\hline & M. oculatus Ruthe, 1862 & Western Palearctic & Psychidae (Lep.) \\
\hline & *M. punctifrons Thomson, 1895 & Western Palearctic & Tenebrionidae (Col.) \\
\hline & *M. radialis Tobias, 1986 & Western Palearctic & Unknown \\
\hline
\end{tabular}

...... continued on the next page 
TABLE 3 (continued)

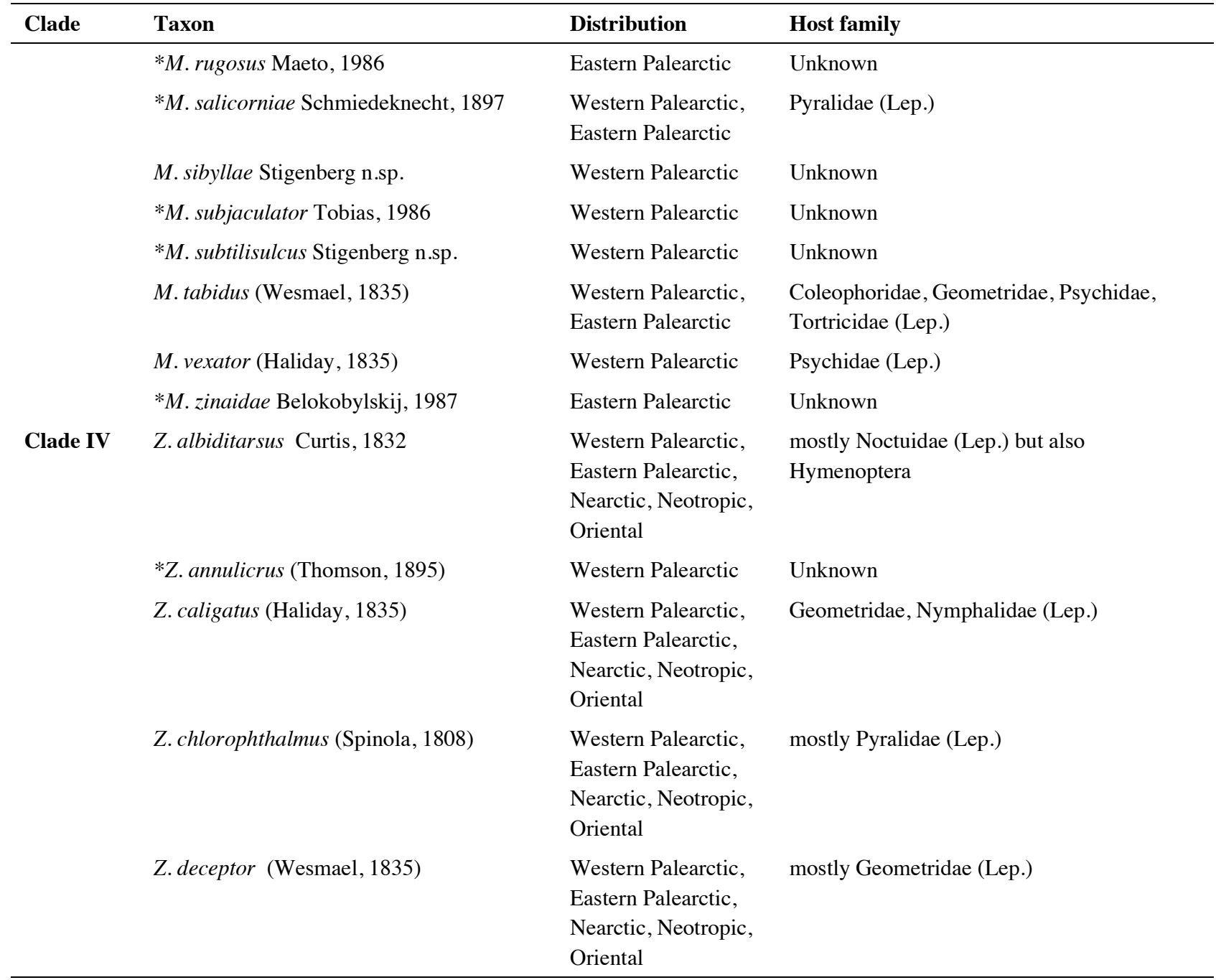

The second lineage (Clade IIB) consists of M. abscissus, M. acerbiavorus, M. limbatus, M. obsoletus, M. pendulus, M. pulchricornis, M. rubens and M. versicolor (Fig. 4) This lineage is characerized by: (1) no dorsope (except M.pendulus, M. abscissus, and M. limbatus); (2) face narrow; (3) mandibles strongly twisted; (4) no tubercle on frons; (5) ovipositor short. It largely corresponds to Maeto's pulchricornis group (except for the colon subgroup), and the rubens group. The first author examined material of M. heliophilus, M. insulicola, M. kurokoi, M. lionotus, M. nodai, M. politutele, M. rufus and M. takenoi and, and can confirm that they all are likely to belong to this lineage (Table 3).

Finally, the third lineage (Clade IIC) consists in our analysis solely of $M$. micropterus. Maeto also placed $M$. salicorniae in his micropterus group but study of material of $M$. salicorniae by the first author indicates that the character that groups them together, the strongly convex face, is most probably a parallelism and not a true synapomorphy. Judging from its morphology, M. salicorniae is more likely to belong to Clade III than to be closely related to M. micropterus (Table 3).

Our Clade III (Fig. 4) includes M. abdominator, M. affinis, M. alborossicus, M. cespitator, M. cis, M. consimilis, M. corax, M.densipilosus, M. eklundi, M. filator, M. hirsutipes, M. jaculator, M. kyushuensis, M. longicaudis, M. longipilosus, M. obfuscatus, M. oculatus, M. sibyllae, M. sulcatus, M. tabidus, and M. vexator. After examining dry-pinned material, the we can confirm that the following species also belong to Clade III: M. acutus, M. albizonalis, M. angiclypealis, M. angustatus, M. breviantennatus, M. brevicauda, M. curvus, M. gotoi, M. ipidivorus, M. jezoensis, M. melanostictus, M. micropilosus, M. nadezhdae, M. nixoni, M. radialis, M. rugosus, M. subjaculator, M. subtilisulcus, and M. zinaidae (Table 3). Clade III is rather heterogeneous morphologically and biologically, and it is quite difficult to find diagnostic morphological characters. The majority of species, however, appear to be 
coleopteran parasitoids. Clade III corresponds to Maeto's corax, hirsutipes and albizonalis groups but also includes some members of his ictericus group, micropterus group, and colon subgroup of the pulchricornis group.

Our Clade IV corresponds to the genus Zele, as circumscribed by van Achterberg and others. Thus far, we have not seen material of any Meteorus species that seems more closely related to Zele than to one of the three Meteorus clades identified by us (Table 3 ).

\section{Species Circumscription}

Our analysis of cryptic species diversity in the Meteorini was largely based on the Swedish SMTP material. A geographically and morphologically diverse sample of 177 specimens of 41 morphospecies was sequenced, selected from more than 900 specimens examined from the SMTP catches. The analysis resulted in the discovery of 12 cryptic species, which were clearly separated genetically although we were not able to distinguish them morphologically, not even after they had been identified in the molecular analysis.

The GMYC analysis indicated the presence of an additional 8 species, most of which were represented by single individuals with slightly deviating $\mathrm{CO} 1$ sequences. We suspect that studies of additional specimens will show that many of these variants do not represent distinct species. Nevertheless, further study of this genetic variation using additional specimens and more markers is clearly warranted.

If our sample is representative, this suggests that cryptic species may constitute $20 \%$ (12 / 59) to $34 \%$ (20 / 59) of the morphologically discoverable species diversity in the Meteorini. These results agree fairly well with those of other molecular studies of hidden species diversity in parasitic wasps (Smith et al. 2008, Fernández-Triana 2010). Smith et al. (2008) showed a 70\% increase in species diversity based on 2.597 specimens and Fernandez-Triana's study (2010), based on more than 12.000 specimens, indicated an increase with $50 \%$.

Quicke (2001) discussed the cryptic species problem and proposed that specimens identified solely by molecular evidence would be labeled as molecularly Recognisable Taxonomic Units (mRTUs). He also pointed out the difficulty of associating existing scientific names with the correct mRTUs and suggested two possible solutions: either a non-argumentative approach, in which mRTUs are arbitrarily assigned to existing species names by the principle of priority (analogous to the first revisor principle), or a parallel approach, in which mRTUs are simply not named using traditional binomial nomenclature.

The problems discussed by Quicke are well exemplified by our study. For instance, consider the case of $M$. pendulus, which is apparently a complex of at least three mRTUs (Figs 4-6). Meteorus pendulus currently has six synonyms meaning that seven scientific names are available for the three mRTUs. Matching the mRTUs to these names will require sequencing of old type material, which may not be possible. A practical approach in cases like this might be to sequence material from the type localities of the described species, and then assign mRTUs to names by matching geographic distributions, if possible, otherwise by arbitrary matching. Once the assignment of mRTUs to existing names has been made, the principle of priority would then be used to permanently link the names to diagnostic DNA sequences of the mRTUs.

Such an approach should be based on molecular study of material from a larger geographic region than the one targeted in this study. For this reason, among other things, we refrain from naming the mRTUs discovered here or trying to match them to existing species names; instead our treatment of the cryptic species is at the level of species complexes.

Which morphological characters are most useful in separating closely related species of Meteorus and Zele? There is considerable intraspecific variation in many characters, particularly in the males. Characters that nevertheless provide reliable diagnostic differences include the structure of the precoxal sulcus and the sculpture of the propodeum (Huddleston 1980). The colour patterns of females are also surprisingly stable, and can often be used for separating otherwise similar species.

Body size is quite variable and can only be used as a rough guide to differentiate species. The shape of the head and its various regions are sometimes diagnostic of species. However, the eyes vary in size among specimens, which can affect the appearance of these characters. If the eyes are small, they converge less strongly ventrally and the head tends to be more transverse in anterior view. Sexual dimorphism in the Meteorini is not pronounced, but males in Meteorus have longer antennae and more flagellomeres than females, which should be born in mind when identifying specimens. The wing venation also tends to vary more in males than in females. 


\section{Evolution of Host Preferences}

Euphorines are well-known adult parasitoids of Coleoptera, Hemiptera, Hymenoptera, Neuroptera, Psocoptera, Orthoptera and Lepidoptera (Huddleston 1980, Shaw 1985 and 1988). They are also known to parasitize on nymphs (Loan 1974). The Meteorini are unique among euphorines in attacking insect larvae. Since adult hosts are very rare in other braconids, it seems likely that the Meteorini have retained the ancestral habit of the Euphorinae.

Čapek (1970) concluded, in his studies of larval morphology of Meteorini, that the ancestral hosts of the tribe were Lepidoptera larvae. In contrast, Shaw (1985) found in the first computerized analysis of Meteorini phylogeny, based on morphological data, that the most recent common ancestor of the Meteorini was likely to have attacked Coleoptera larvae. More recent studies, however, support the older idea of the ancestral hosts belonging to Lepidoptera (Li et al. 2003, Zitani 2003, Pitz et al. 2007, Boring 2010).

Our molecular results indicate that all coleopteran parasitoids in the Meteorini belong to a single clade, Clade III, which is nested inside other clades that are specialized on Lepidoptera. Counting both the species included in our phylogenetic analysis, and other species of which we have seen material, we have studied about $20 \%$ of the Meteorini species, mostly from the Western Palearctic but also from other parts of the world. In this material, all coleopteran parasitoids appear to belong to Clade III. Therefore, we are fairly confident in concluding that the Meteorini originally attacked lepidopteran hosts, and that coleopteran parasitism is secondary.

Inferring the evolution of host preferences in more detail is difficult both because of the lack of data and because many of the published host records are erroneous due to incorrect identification of the Meteorus species. Nevertheless, some patterns seem to appear from the current analysis and will be discussed below. New host data based on reared material, which could be used in a more accurate future analysis, will be presented elsewhere (Stigenberg \& Shaw in prep.).

Examining host preferences at the family level, we note that M. ictericus and M. ruficeps, which form Clade I (Fig. 4), mostly parasitize Tortricidae (Huddleston 1981, Yu et al. 2005). Clade II (Table 3, Fig. 7) is a group that parasitizes several lepidopteran families, the most frequent being the Noctuidae, Geometridae and Tortricidae (Huddleston 1981; Yu et al. 2005). The members of Clade II share morphological traits such as slender body, large ocelli, twisted mandibles and long ovipositor, which may partly represent adaptations to parasitism of lepidopteran larvae. An interesting feature in this clade is the gregarious lifestyles of M.rubens (Nees 1811) and M. acerbiavorus (Belokobylskij et al. 2011). This is shared also by M heliophilus Fischer 1970 and several tropical species, including the Costa Rican species M. papiliovorus (Zitani 1997) and M. oviedoi (Shaw et al. 2005). Based on morphological features, all three species are likely to belong to Clade II. Gregariousness is likely to be a derived character in Clade II.

The hosts of Clade III (Table 3, Fig. 9) include both Coleoptera and Lepidoptera larvae. The attacked species belong to a wide range of families: hosts within Lepidoptera include members of the Noctuidae, Geometridae, Pyralidae and Tineidae, while coleopteran hosts include members of the Scolytidae, Cerambycidae, Melandryidae, Tenebrionidae and Cisidae. It is difficult to infer the ancestral host of Clade III from available data. There are no host records on either M. eklundi or M. filator, both basal lineages in the clade, but since members of the rather basal species M. oculatus are parasitoids of Lepidoptera, it is possible that this is the ancestral host. Due to the poor resolution of relationships within Clade III, it is unclear whether parasitism of Coleoptera evolved once or repeatedly within the lineage. The members of Clade III that parasitize Coleoptera larvae tend to have small ocelli, less twisted mandibles, and long and slender ovipositors.

Members of Zele, which form Clade IV (Fig. 4), mostly parasitize Geometridae, Noctuidae, Tortricidae, and Pyralidae. The shape of the ovipositor varies within Zele. The species occurring in the Western Palearctic have moderately short and sturdy ovipositors. It is interesting to notice that the association with hosts in the Tortricidae recurs in three of the four major Meteorini clades. It may possibly indicate that the ancestral Meteorini hosts belonged to this family. 


\section{Taxonomic Treatment with Keys}

\section{The Tribe Meteorini Cresson 1887}

Cresson, 1887, Transactions of the American Entomological Society, Supplement: 55, 60

Syn.: Zelini Ashmead, 1900; Petiolarini Szépligeti, 1904; Zemiotini van Achterberg, 1976.

Diagnosis: Occipital carina complete, 3rd segment of labial palpi reduced or absent, anterior tentorial pits deep, medium sized to large; mesopleuron more or less protruding antero-dorsad; medial lobe of mesoscutum more or less rounded anteriorly; vein $\mathrm{r}-\mathrm{m}$ of fore wing present; forewing with three submarginal cells, the second submarginal cell generally higher than long; petiolar tergum clearly petiolate, narrow at base and widened apically, spiracles on petiolar tergum situated submedially; ovipositor always exserted.

\section{Key to European genera of Meteorini:}

$1 \quad$ Marginal cell of hind wing widened apically (Fig. 10a), at least apical half of 3rd and following abdominal tergites setose (Fig.

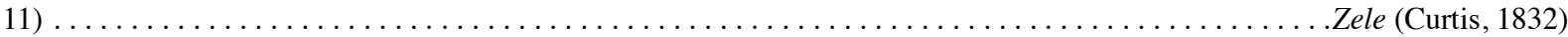

- Marginal cell of hind wing subparallel or narrowed apically (Fig. 10b), 3rd and following abdominal tergites with a single row of setae $($ Fig. 12) . . . . . . . . . . . . . . . . . . . . . . . . . . . . . . . . Meteorus

\section{Key to European species of Zele:}

1 Vein cu-a of forewing antefurcal (Fig. 13a) Z. chlorophthalmus (Spinola, 1808)

- $\quad$ Vein cu-a of forewing postfurcal (Fig. 13b) .3

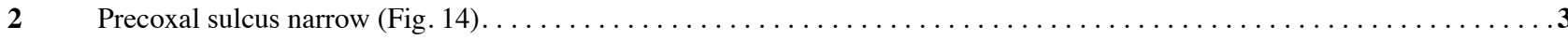

- $\quad$ Precoxal sulcus wide, not always strongly rugose (Fig. 15) (albiditarsus Curtis s.1.) ......................

3 Ovipositor short, 1.5 times petiolar tergum, (ovipositor sheath $0.19-0.28$ times fore wing) .... Z. caligatus (Haliday, 1835)

- $\quad$ Ovipositor long 2.5 times petiolar tergum (ovipositor sheath $0.38-0.39$ times fore wing) .... Z. annulicrus (Thomson, 1895)

4 Length of fore leg tibial spur 0.4-0.5 times fore basitarsus, fore femur swollen, 5.0-6.0 times as long as wide (Fig. 16) ..... .

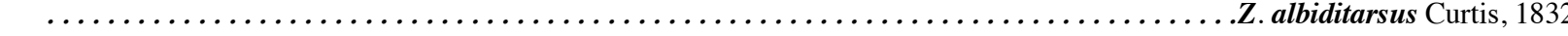

- $\quad$ Length of fore leg tibial spur 0.2-0.4 times fore basitarsus, fore femur slender, 6.5-9.0 times as long as wide (Fig. 17) . . . .

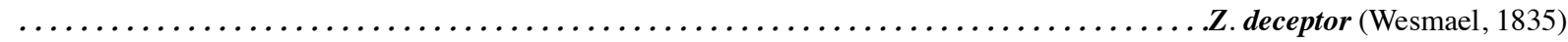

\section{Key to European species of Meteorus:}

1 
Body entirely black and rugose (Fig. 31); malar space of $q$ equal or slightly longer than basal width of mandible; antennal arti-

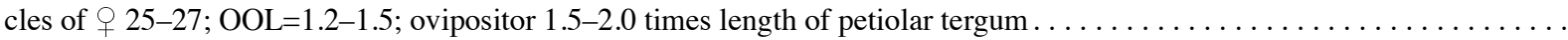

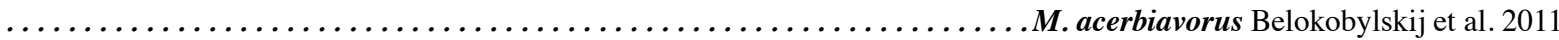

Clypeus distinctly protruding, rounded (Fig. 105); OOL=0.5 (Fig. 32); setae on clypeus scattered; temples strongly contracted (Fig. 32); length of ovipositor about 1.5-2.0 times length of petiolar tergum; antennal articles of $\varnothing$ 31-33 $\ldots \ldots \ldots \ldots$

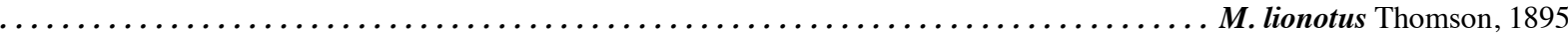
Clypeus with peculiar cushion-like shape, protruding but "flattened" (Fig. 28); OOL=1.5; setae on clypeus dense and short; temples rounded; ovipositor 1.5-2.0 times length of petiolar tergum; antennal articles of $\bigcirc$ 29-33.

.M. pulchricornis (Wesmael, 1835)

Ovipositor slender, length 1.6-2.5 times petiolar tergum; eyes not protruding (Fig. 113); temples rounded; clypeus as wide as face; base of petiolar tergum brownish or blackish; antennal articles of $q 27-30 \ldots \ldots \ldots$. . . obsoletus $($ Wesmael, 1835) Ovipositor rather thick and 1.4-2.0 times length of petiolar tergum; eyes protruding (Fig. 132); base of petiolar tergum often pale yellow, almost translucent basally (Fig. 33); antennal articles of $q$ 29-33............ M. versicolor (Wesmael, 1835)

Face forming a "shelf" (Fig. 34); wings short and narrow, often infumate (Fig. 35); face strongly convex; propodeum strongly reticulate-rugose, without distinct carinae; occipital carina interrupted medio-dorsally; body compact and dark brown (Fig. 36); antennal articles of $\varnothing 23-26(\delta 24-28)$ OOL $=3 \ldots \ldots \ldots \ldots \ldots \ldots \ldots \ldots \ldots \ldots \ldots \ldots \ldots$ micropterus $($ Haliday, 1835) Face without such shelf; wings longer and wider; body colour varying, often legs yellow; antennal articles of $q$ 21-34 . . 10

Mandibles stout, not twisted, clypeus rather flat (Fig. 98); propodeum with strong anterior transverse carina and medial longitudinal carina (Fig. 37); vein m-cu of fore wing distinctly antefurcal or interstitial; antennal articles of $q$ 21-27; pterostigma

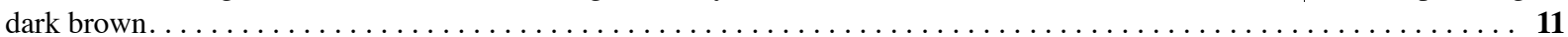
Mandibles small and more or less twisted (Fig. 86); clypeus rather protruding; propodeum without anterior transverse carina or with a median longitudinal carina (Fig. 38); vein m-cu of fore wing (sub) interstitial or postfurcal (Fig. 39), exceptionally shortly antefurcal; antennal articles of $q 24-34$; pterostigma variable $\ldots \ldots \ldots \ldots \ldots \ldots \ldots \ldots \ldots \ldots \ldots \ldots$

11 Clypeus wide and flat; face narrow, its minimum width about equal to its height (Fig. 98); eyes large protruding and converging; antennal articles slightly longer than broad and sturdier, basal articles lighter in colour than apical ones (Fig. 40), antennal articles of $q$ 21-25 ( $₫ 27-31$ ); length of ovipositor 2 times petiolar tergum; $\mathrm{OOL}=2.5-3 \ldots \ldots$. . M. filator (Haliday, 1835) Note: Males of $M$. filator have wider face and smaller eyes, very easy to go wrong here but check antennal length.

- $\quad$ Clypeus narrow and protruding; face wider, its minimum width about 1.5 times its height (Fig. 96); eyes smaller less converging; antennal articles long and slender, base of antenna concolorous with apex or nearly so (Fig. 41), antennal articles 26-27; length of ovipositor 1.5 times petiolar tergum; $\mathrm{OOL}=2.5-3 \ldots \ldots \ldots \ldots \ldots \ldots \ldots \ldots \ldots \ldots \ldots$. eadyi Huddleston, 1980

$\mathrm{OOL}=1.5-2$; body frequently yellow or pale brown, sometimes black; pterostigma yellowish or pale brown, elongated (Fig. 30); subapical antennal articles of $q$ distinctly longer than wide; ovipositor 1.0-2.0 times petiolar tergum; antenna of $q$ with 30-34 articles; precoxal sulcus narrow; vein m-cu of fore wing distinctly postfurcal, usually with about 2 times width of vein (Fig. 30); vertex reddish near eyes; hind femur yellowish, as tibia; frons convex medially; hind coxa with some rugosity .....

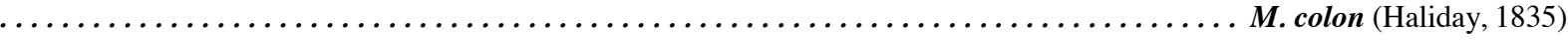
$\mathrm{OOL}=2-3$ times; body mostly black, legs and details on head yellow to brown; pterostigma varying from brown to yellow (Fig. 42, 43); ovipositor 2.0-4.0 times petiolar tergum; antenna of $q$ with $24-30$ articles; precoxal sulcus wider $\ldots \ldots \ldots 13$

13 Pterostigma except basal $3^{\text {rd }}$ dark brown (Fig. 42); subapical antennal articles of $\odot$ subquadrate (Fig. 44); hind femur more or less dark brown or infuscate apically; antennal articles of $q$ 24-29 (o 25-30); length of ovipositor 2.5-3.0 times petiolar tergum, 5.5-5.6 times fore wing; eye 1.9-2.0 times as long as temple in dorsal view; length of malar space 0.6 times basal width of mandible; precoxal sulcus wide .................................. cinctellus (Spinola, 1808)

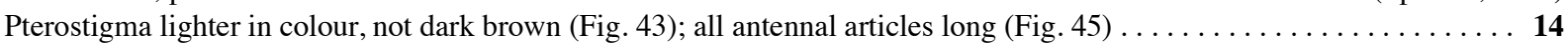

14 Mesosoma with dark reddish-brown pattern (Fig. 46); ovipositor 2.4 times petiolar tergum and 5.3 times fore wing; eye about 1.5 times as long as temple in dorsal view; malar space 0.6 times basal width of mandible; second submarginal cell elongated, postfurcal; face equally broad as high; $\mathrm{OOL}=1.9$; antenna of $q$ with $27-28$ articles. . . . . . . M. stenomastax sp. nov. Mesosoma dark-black, not reddish but sometimes with a lighter yellowish spot $\ldots \ldots \ldots \ldots \ldots \ldots \ldots \ldots \ldots \ldots$

Ovipositor long, 2.0-4.0 times petiolar tergum (Fig. 47); vein mc-u of fore wing interstitial or postfurcal (Fig. 43); third abdominal tergum yellow-brown, lighter than following tergum; median longitudinal carinae on propodeum visible to various degrees; antennae ( $($ ) with 27-30 articles; no orange-red markings adjacent to precoxal sulcus . . . M. tenellus Marshall, 1887 Ovipositor short, 1.6-2.0 times petiolar tergum (Fig. 48); vein mc-u of fore wing interstitial or antefurcal (Fig. 49); median longitudinal carinae on propodeum weak or obsolescent; antennae ( $($ ) with 27 articles; some specimens have orange-red markings on both sides of precoxal sulcus $\ldots \ldots \ldots \ldots \ldots \ldots \ldots \ldots \ldots \ldots \ldots \ldots \ldots \ldots \ldots \ldots \ldots \ldots \ldots$ artocercus sp. nov. 
Third abdominal tergum smooth (Fig. 50); petiolar tergum slender or variable; dorsopes situated medially (Fig. 21); face variable; body size smaller and body colour varying; antennae with about 30 articles or less $\ldots \ldots \ldots \ldots \ldots \ldots \ldots \ldots$ Third abdominal tergum striated, petiolar tergum wide and stout (Fig. 51); dorsopes situated basally; face transverse; body

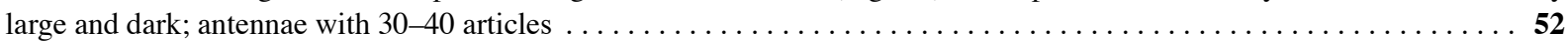

17 Hind tibia wide, parallel-sided and medially about as wide as apex of femur (Fig. 52); eyes strongly convergent, large and strongly protruding (Fig. 114); mandibles slightly twisted; face as wide as broad; antennal articles of 9 30-33; OOL about 2.5

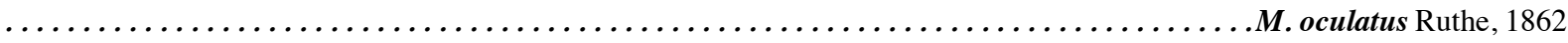
Hind tibia gradually widened to apex but not as wide as femur (Fig. 53) $\ldots \ldots \ldots \ldots \ldots \ldots \ldots \ldots \ldots \ldots \ldots$

18 Small species, less than about $3.5 \mathrm{~mm}$ in length. Ovipositor about two times petiolar tergum. Seldom collected (except $M$. vex-

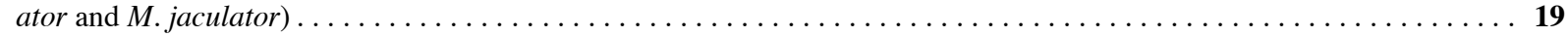
Larger species, more than about $3.5 \mathrm{~mm}$ in length. Ovipositor length variable. More frequently collected $\ldots \ldots \ldots \ldots 31$

19 Antennae with 20-23 articles, clypeus sometimes with a ventral notch (Fig. 91); (if eyes large and greatly converging $M$. vexator (Fig. 133) or if ovipositor 3-4 times petiolar tergum $M$. jaculator cf. couplet 22$) \ldots \ldots \ldots \ldots \ldots \ldots \ldots \ldots \ldots$ Antennae with more articles, clypeus without a notch .

Clypeus rather wide (Fig. 87), malar space very short; hind coxa punctate; ovipositor almost twice petiolar tergum; propodeum

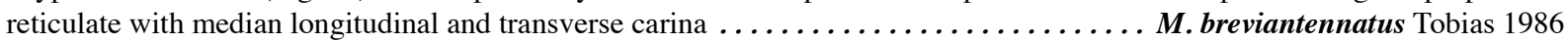

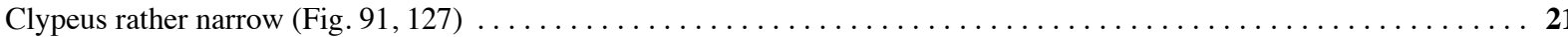

21 Malar space almost as wide as mandible base; eyes only slightly converging, head subcubic (Fig. 91); hind coxa often only partly rugose; ovipositor 2 times petiolar tergum ................................ (Bouché, 1834) Malar space slightly shorter than mandible base; eyes converging slightly, head somewhat transverse (Fig. 127); hind coxa punctate; ovipositor 3.5 times length of petiolar tergum....................... subjaculator Tobias 1986

24 Frons between ocelli and eyes with distinct punctures (Fig. 55); clypeus narrower than face (Fig. 119); ovipositor downcurved; OOL $=4$; temple distinctly widened ventrally (Fig. 55); $q$ antennal articles 25-27; head subcubic; hind coxa more or less darkened; eyes protruding, cheeks also protruding; mandibles stout; very small in body size, about $2 \mathrm{~mm} \ldots \ldots \ldots \ldots$.

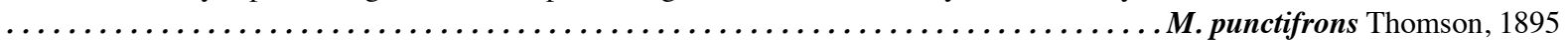
Frons between ocelli and eyes without distinct punctures, sometimes small indistinct punctures; clypeus variable; head not

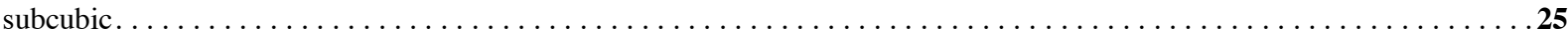

Clypeus as wide as face, lateral corner of clypeus reaching inner level of eye (Fig. 88); OOL=3; vein m-cu antefurcal; antennal

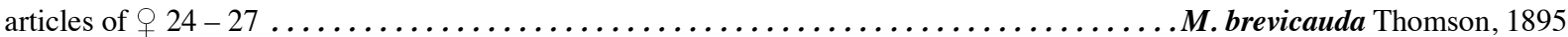
Clypeus small and square (Fig. 109); OOL about 3; frons between ocelli and eyes with small indistinct punctures; vein m-cu distinctly postfurcal; antennal articles of $q 27 \ldots \ldots \ldots \ldots \ldots \ldots \ldots \ldots \ldots \ldots \ldots \ldots \ldots$ micropilosus Tobias, 1986

Marginal cell very narrow (Fig. 56); mesopleuron, pronotum, propodeum and coxae very rugose, whole specimen gives a

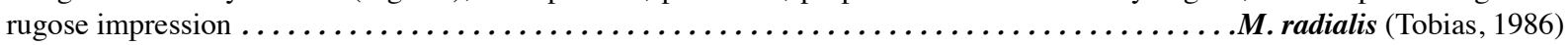
Marginal cell as in Figs 39, 42, 43; mesopleuron, pronotum, propodeum and coxae if rugose then only partially $\ldots \ldots \ldots 27$

Fore wing vein 1-SR+M only partial (Fig. 57); antennae of $q$ with 26-28 articles; eyes large, protruding and converging (Fig. 84); ovipositor 2.5 times petiolar tergum; hind coxae rugose, clypeus as wide as face . ...M. alborossicus (Lobodenko, 2000) Fore wing vein 1-SR+M fully sclerotized (Fig. 58); antennae of $\bigcirc$ 20-25 articles; eyes large, protruding and strongly converging (Fig. 133); ovipositor 2-3 times petiolar tergum; hind coxa slightly rugose; clypeus broader than face. . .

M. vexator (Haliday, 1835)

29 Head rounded; fore wing vein $\mathrm{r}$ equal in length to 3-SR; hind coxae largely smooth .. M. angiclypealis van Achterberg, 2001 Head transverse; fore wing vein $\mathrm{r}$ distinctly shorter than 3 -SR; hind coxae rugose dorsally $\ldots \ldots \ldots \ldots \ldots \ldots$

30 Antennal articles of $q$ 18-22; propodeum with distinct carinae, smooth inbetween; clypeus rather flat and as wide as face (Fig.

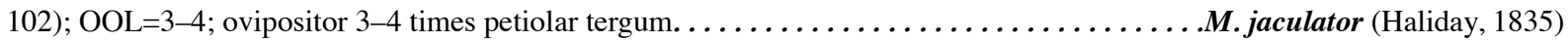


Antennal articles of $q$ 24-26; propodeum with indistinct carinae, weakly rugose; clypeus as wide as face, distinctly separated from face, convex (Fig. 125); $\mathrm{OOL}=3-3.5$; ovipositor $3.5-4$ times petiolar tergum ............... sibyllae sp. nov

Petiolar tergum with two dark black spots (Fig. 59). Whole body punctate (face, pronotum, mesonotum etc); OOL=1.5 . . . .

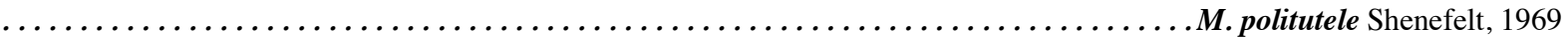
Petiolar tergum without any spots. Body with other surface structure $\ldots \ldots \ldots \ldots \ldots \ldots \ldots \ldots \ldots \ldots \ldots \ldots \ldots \ldots$

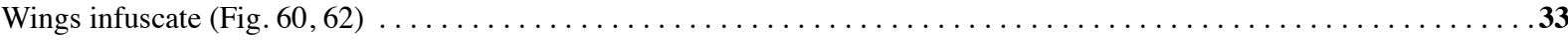

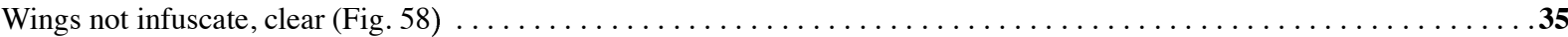

33 Ovipositor short, thick and down-curved, length equal to petiolar tergum; length of malar space of $\odot$ 1.5-2.0 times ( o $^{\lambda} 1.3-1.4$ times) basal width of mandible; $q$ antennae with pale band (Fig. 61); second submarginal cell of fore wing strongly narrowed anteriorly (Fig. 62); $q$ antenna $32-33$ articles $(\delta$ up to 36 ); $\mathrm{OOL}=1.5-2.0 \ldots \ldots \ldots \ldots \ldots$. . . consimilis (Nees, 1834)

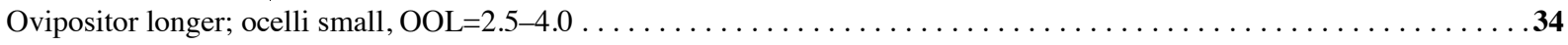

34 Ovipositor about twice the length of petiolar tergum and slightly down-curved (Fig. 63); OOL=3.5-4.0; malar space slightly shorter than mandible base; antennal articles of $q 22-26(\delta 25-31) \ldots \ldots \ldots \ldots \ldots \ldots \ldots$. abdominator (Nees, 1811) Ovipositor about three times the length of petiolar tergum, straight (Fig. 64); OOL=2.5; malar space 0.5 times mandible base;

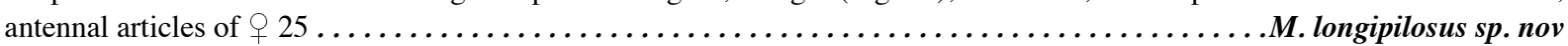

Face wider than high, width seldom equal to height (except M. eklundi. Fig. 97) (Fig. 106); ocelli often small; OOL=2-3 ..36 Face not wide, mostly higher than wide or height and width equal (Fig. 122); ocelli often large; OOL $=1-2 \ldots \ldots \ldots \ldots 44$

Ovipositor very long, 2.5-4.0 times petiolar tergum (if 2.5 then ovipositor sheaths with long erect hairs M. salicorniae, cf. cou-

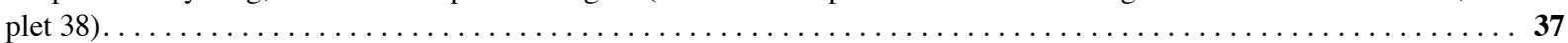

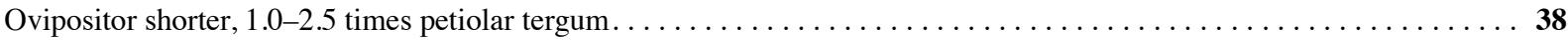

Face transverse, wider than high, and eyes small (Fig. 106); antennal articles of $q$ 29-32; ovipositor 4.5 times petiolar tergum

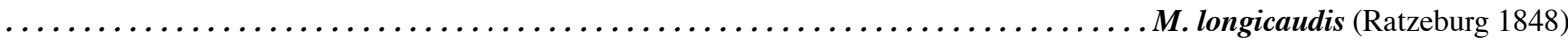
Face not so transverse and eyes large (Fig. 89); antennal articles of $q$ 23-27; ovipositor 3-4 times petiolar tergum........

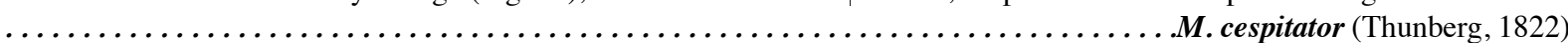

Ovipositor sheath with long erect hairs, the hairs two times the width of the sheath (Fig. 65); face very transverse and eyes small (Fig. 124); antennal articles 33-35; ovipositor 2.5 times petiolar tergum. . . . . . M. salicorniae Schmiedeknecht, 1897 Ovipositor sheath with normal hairs; antennal articles of $\rho_{+} 23-32$; ovipositor $1.5-2.5$ times petiolar tergum $\ldots \ldots \ldots . .39$

39 Clypeus slightly protruding (Fig. 66); hind coxae smooth, punctuate; face 1.5 times as wide as high (Fig. 112); subapical antennal articles of $q$ robust, squarish; length of ovipositor 1.7-2.0 times petiolar tergum; clypeus often similarly brownish as face

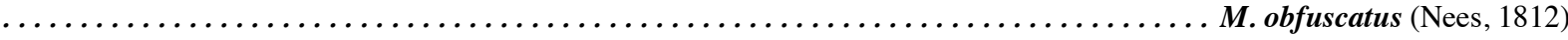
Clypeus rather flat and wide (Fig. 103); hind coxae rugose (except $M$. kyushuensis) $\ldots \ldots \ldots \ldots \ldots \ldots \ldots \ldots \ldots \ldots$

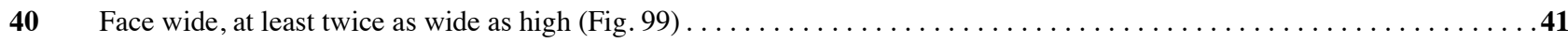

41 Clypeus flat and wide, mandibles large, not twisted (Fig. 99); pronotum yellowish, contrasting with dark mesoscutum (Fig. 67); tarsal claws very long and slender; length of ovipositor $2.2-2.5$ times petiolar tergum; $q$ antennal articles $26-30 \ldots \ldots$.

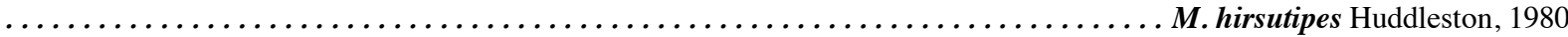
Clypeus flat but not so wide, narrower than width of face (Fig. 103); mandibles large, slightly twisted; pronotum orangebrown; tarsal claws not so long; ovipositor about twice as long as petiolar tergum; $ᄋ$ antennal articles 23; colour of face orange M. kyushuensis Maeto 1988 Precoxal sulcus narrow (Fig. 69); ovipositor 1.6 times petiolar tergum and clypeus as wide as face . .M. subtilisulcus $\mathbf{s p . ~ n o v . ~}$

43 Clypeus broad but narrower than face, punctate and flat but with ridges forming a sinuate ventral border (Fig. 95); face broader than high; ovipositor 2 times length of petiolar tergum; antennal articles 28 , all articles longer than wide; OOL=2.5 ......

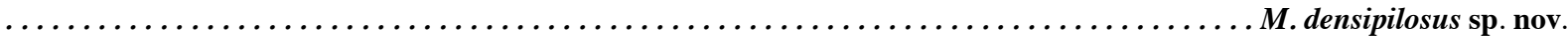
Clypeus narrow but as wide as face (Fig. 96); face almost equally broad as high; ovipositor 1.6 times petiolar tergum, slender and slightly bent downward; antennal articles of $q 22-25$, stout, half of them quadrate; $O O L=3 \ldots \ldots$. M. eklundi sp. nov. Note: If face rugose, rugosity extending down on clypeus, ovipositor straight, antennae not quadrate but longer than wide ...

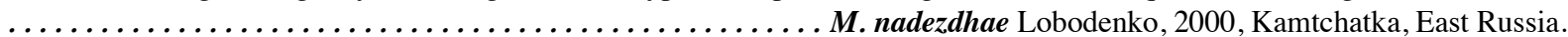

44 Laterope wide and rugose (Fig. 70); pit in front of anterior ocelli (Fig. 71); OOL=1-2; length of ovipositor 1.5-3 times petiolar tergum; if about 2 times then propodeum largely smooth and at most with strong transverse carina; hind coxae slender (icteri- 
cus and ruficeps) or rugose (abscissus, limbatus).

Laterope wide but not so rugose (Fig. 72) or not wide but rugose; mostly no pit in front of anterior ocelli but sometimes a linear impression; $\mathrm{OOL}=1.0-2.4$; length of ovipositor 1.0-3.5 times petiolar tergum; hind coxa reticulate-punctate $\ldots \ldots \ldots .48$

Clypeus smoothly arched and/or rather flattened, densely covered with setae (Figs 73, 75); ovipositor 1.5-2.0 times petiolar tergum; hind coxae rugose and yellow to brown; $q$ antennal articles 30-33; precoxal sulcus wide and strongly reticulate-

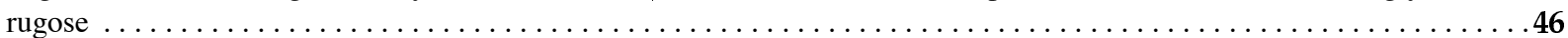
Clypeus distinctly protruding, with scattered setae (Fig. 74); ovipositor 2.5-3.0 times petiolar tergum; hind coxa only dorsally (dorso-laterally) rugose, yellowish; pterostigma pale yellowish; + antennal articles 26-33; precoxal sulcus narrow .... 47

OOL=1.5-2.0; eyes converging (Fig. 122); occiput of $q$ usually largely black; medio-transverse propodeal carina stronger than other sculpture; subapical antennal articles of $q$ about as wide as long; eyes nearly touching clypeus; antennal articles of $q$ 26-

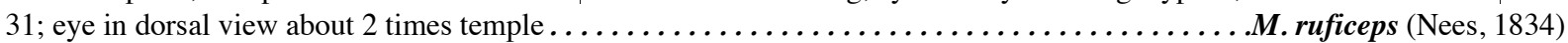
$\mathrm{OOL}=1.0-1.5$; eyes not strongly converging (Fig. 100); occiput of $\circ$ usually yellowish; transverse propodeal carina as weak as other rugae; subapical antennal articles of $q$ distinctly longer than wide; distance wider between eye and clypeus; apex of hind tibia and of hind basitarsus infuscate; antennal articles of $\$ 26-33$; eye in dorsal view 2-3 times temple.

M. ictericus (Nees, 1811)

48 Frons with a blunt tubercle in front of anterior ocellus; propodeum usually without medio-longitudinal carina, its transverse carina may be strongly raised and other sculpture coarser; ovipositor 1.5 times petiolar tergum; antennal articles of $q$ 25-31

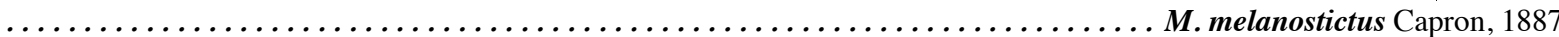

Length of ovipositor 1.5-2.0 times petiolar tergum; $\mathrm{OOL}=1.0-1.5$; ovipositor straight and thick; face slightly broader than high; apex of hind tibia usually distinctly darkened; antennal articles of $\bigcirc$ 30-35 (ô up to 37) ... M. pendulus (Müller, 1776) Length of ovipositor 2.0 times petiolar tergum; $\mathrm{OOL}=1.7-2.0$; precoxal sulcus wide, strongly reticulate rugose; face 1.5-2 times wider than high; antennae up to 36 articles.

M. rufus (De Geer, 1778)

Malar space about 0.6 times basal width of mandible; precoxal sulcus wide; clypeus protuberant; ovipositor stout, 2.5-3 times length of petiolar tergum; $\mathrm{OOL}=2$; antennal articles of $q 28-31 \ldots \ldots \ldots \ldots \ldots \ldots \ldots$. tabidus $($ Wesmael, 1835) Malar space about 0.2-0.5 times basal width of mandible (Fig. 83); precoxal sulcus narrow; clypeus not strongly protuberant; ovipositor slender, $2.5-3.5$ times length of petiolar tergum; $\mathrm{OOL}=1.5-2.0$; antennal articles of $q 27-30$. .

Temples shorter than eyes (Fig. 76); clypeus wide; mandibles large and not twisted; third abdominal tergum only with a few

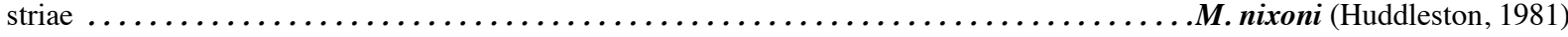
Temples longer than eyes (Fig. 77); clypeus narrow; mandibles short and apically twisted; third abdominal tergum with abundant striae (Figs 51,78).

53 Antennal articles of $q$ 31-36; petiolar tergum almost as broad at its middle as at the apex, flat and sculpture reticulate (Fig. 78) M. sulcatus (Haliday, 1835)

Antennal articles of $q$ 39-41; petiolar tergum gradually narrowing from apex to base, sculpture of petiolar tergum striate and

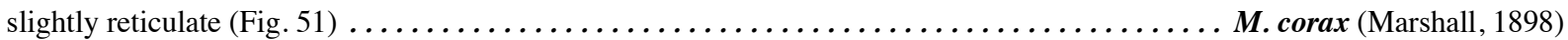



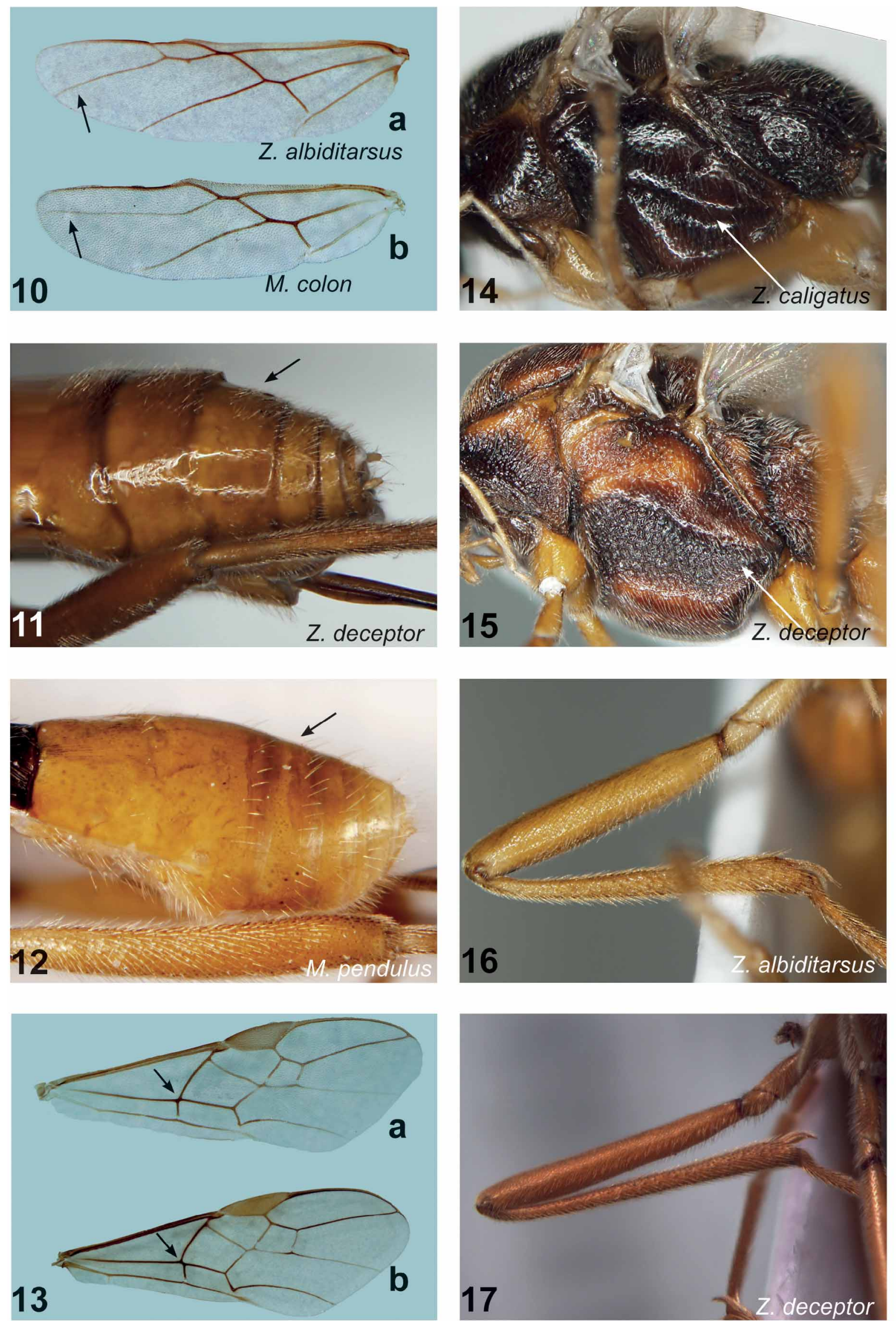

FIGURES 10-17. Characters used in key to species. 10a) hind wing of Z. albiditarsus, 10b) hind wing of M. colon, 11) metaoma of $Z$. deceptor, 12) metasoma of $M$. pendulus, 13a) fore wing of $Z$. chlorophthalmus, $13 \mathrm{~b}$ ) fore wing of $Z$. albiditarsus, 14) mesosoma of Z. caligatus, 15) mesosoma of Z. deceptor, 16) fore femur and tibial spur of $Z$. albiditarsus, 17) fore femur and tibial spur of $Z$. deceptor. 

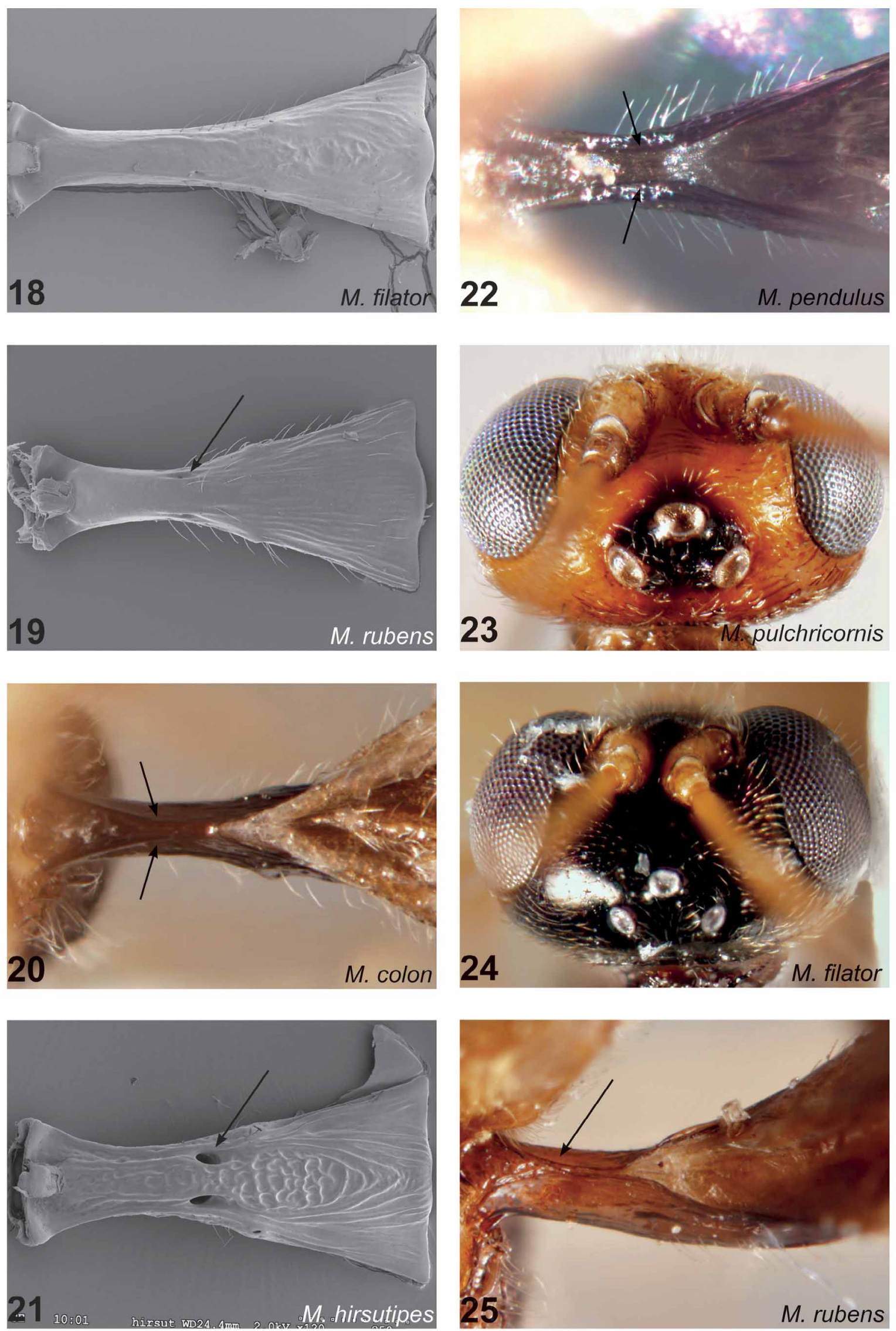

FIGURES 18-25. Characters used in key to species. 18) dorsal view of the petiolar tergum of M. filator, 19) dorsal view of the petiolar tergum of M. rubens, 20) ventral view of the petiolar tergum of M. colon, 21) dorsal view of the petiolar tergum of $M$. hirsutipes, 22) ventral view of the petiolar tergum of $M$. pendulus, 23) dorsal view of the head of $M$. pulchricornis, 24) dorsal view of the head of M.filator, 25) ventral view of the petiolar tergum of M.rubens. 

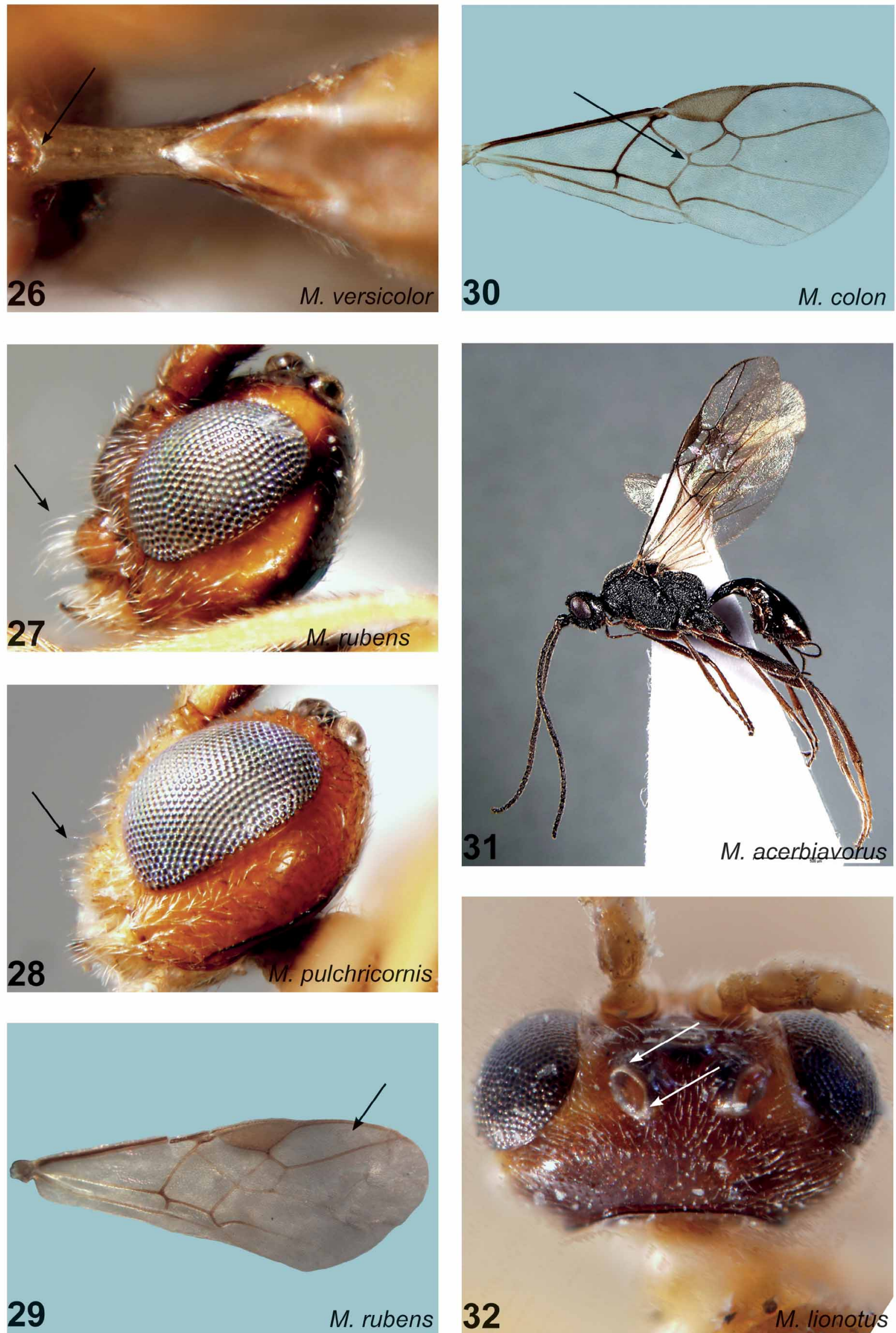

FIGURES 26-32. Characters used in key to species. 26) ventral view of the petiolar tergum of M. versicolor, 27) head in lateral view of $M$.rubens, 28) head in lateral view of $M$. pulchricornis, 30) fore wing of $M$. colon, 31) habitus of $M$. acerbiavorus, 32) dorsal view of the head of M.lionotus. 

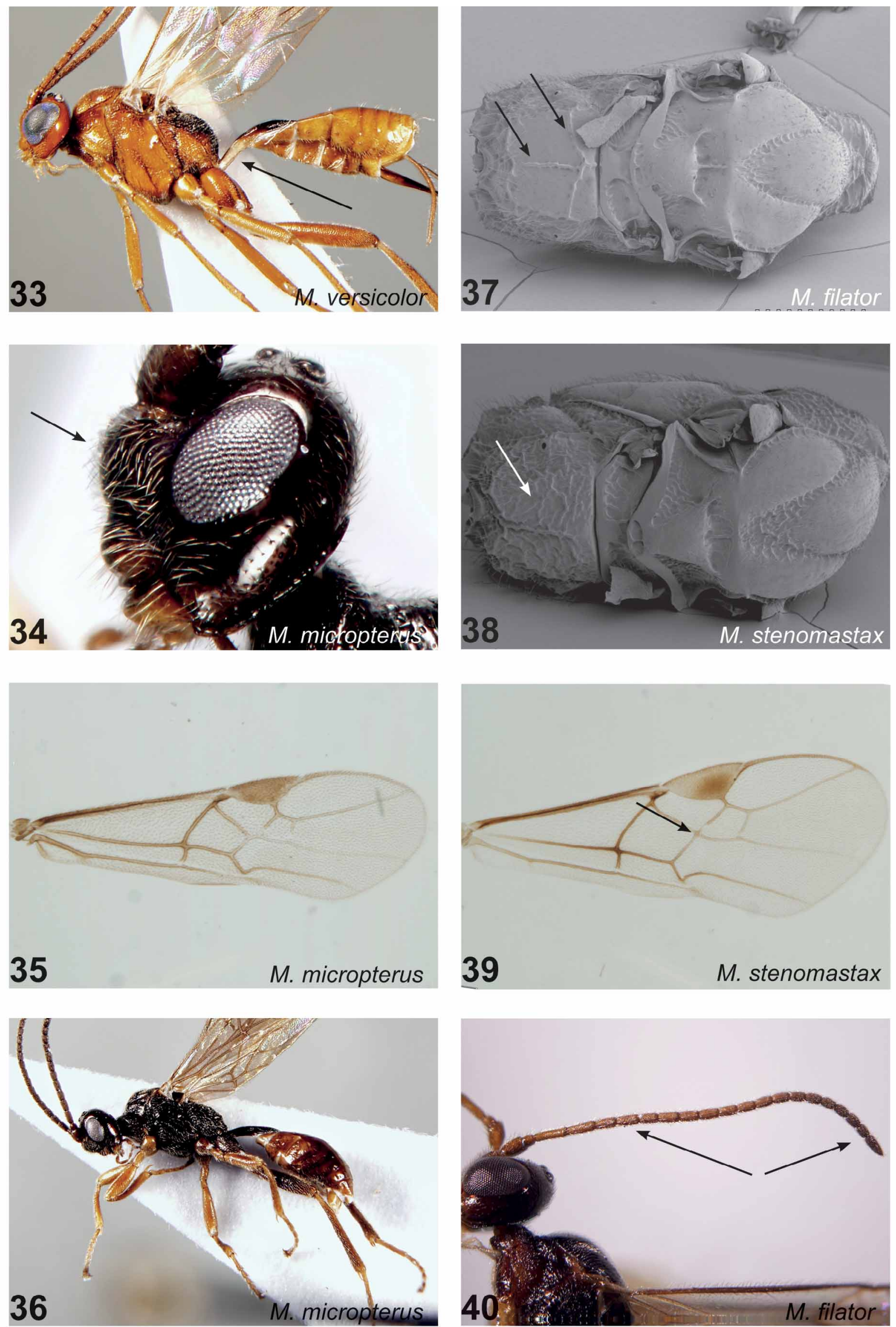

FIGURES 33-40. Characters used in key to species. 33) habitus of $M$. versicolor, 34) head in lateral view of $M$. micropterus, 35) fore wing of M. micropterus, 36) habitus of M. micropterus, 37) dorsal view of the mesosoma of $M$. fialtor, 38) dorsal view of the mesosoma of M. stenomastax sp. nov., 39) fore wing of $M$. stenomastax sp. nov., 40) antennae of M. filator. 

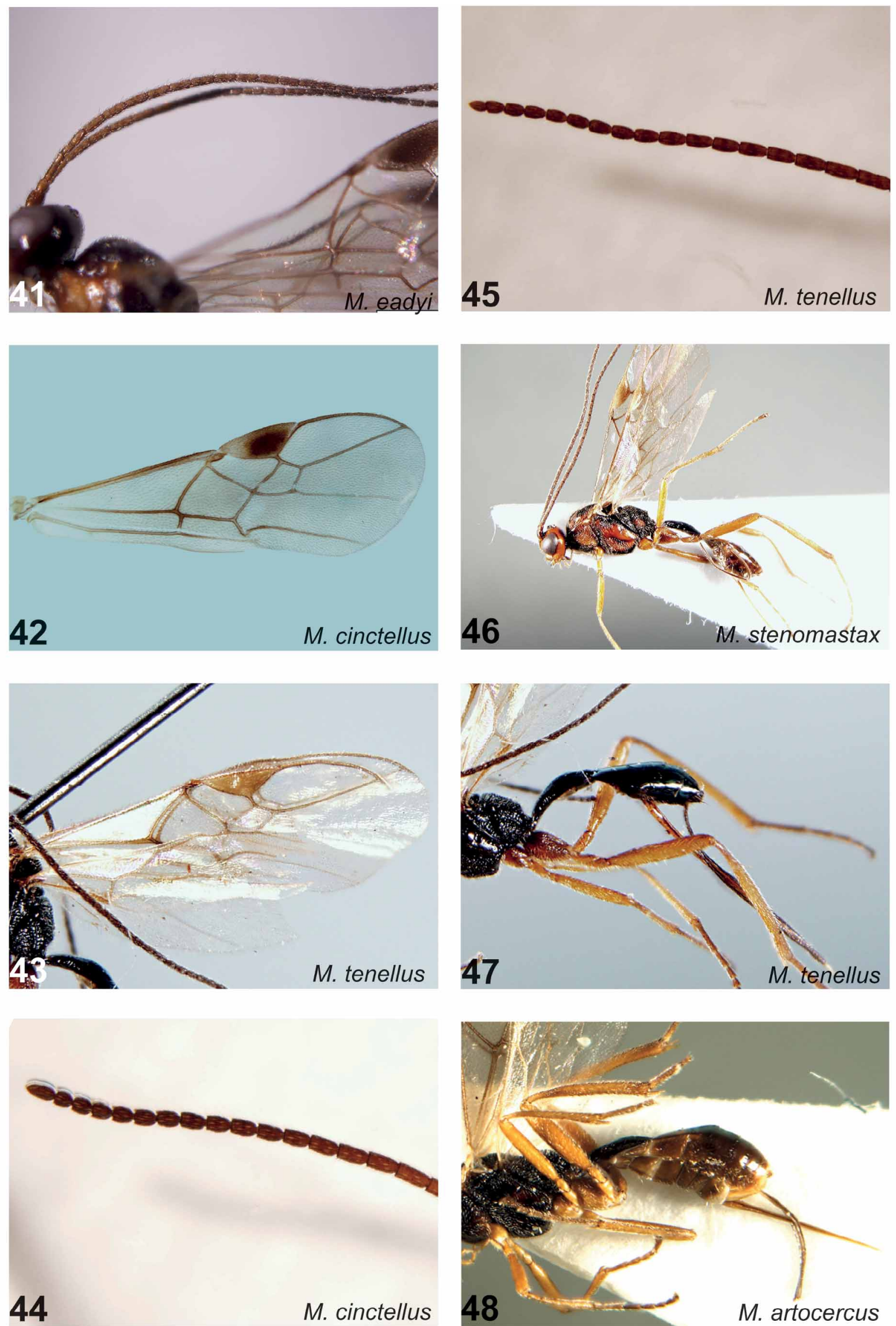

FIGURES 41-48. Characters used in key to species. 41) antennae of $M$. eadyi, 42) fore wing of $M$. cinctellus, 43) fore wing of $M$. tenellus, 44) antennae of $M$. cinctellus, 45) antennae of M.tenellus, 46) habitus of M. stenomastax sp. nov., 47) metasoma of M.tenellus, 48) metasoma of M. artocercus sp. nov. 

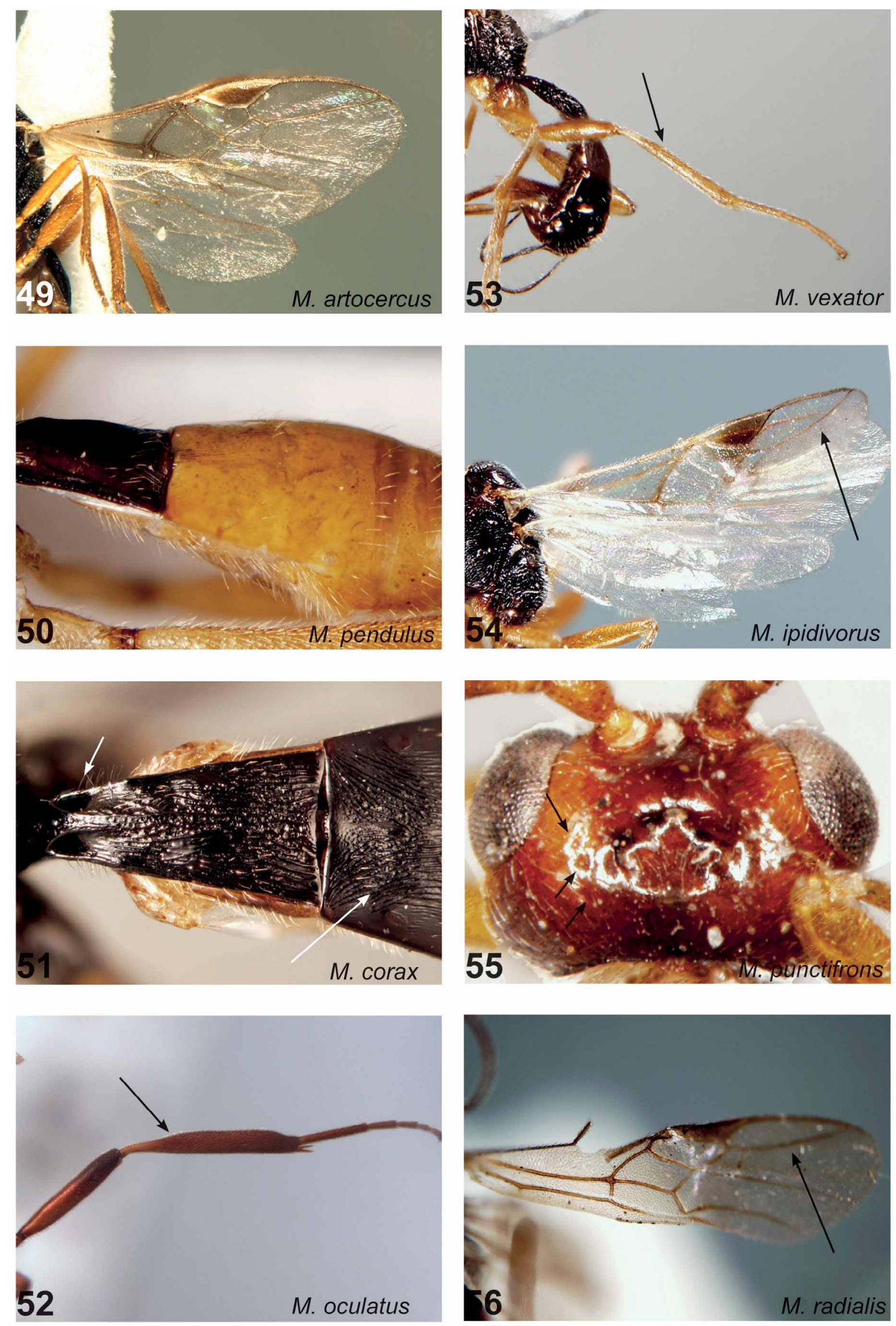

FIGURES 49-56. Characters used in key to species. 49) fore wing of M. artocercus sp. nov., 50) metasomal tergites two and three of M.pendulus, 51) metasomal tergites two and three of M. corax, 52) hind leg of M. oculatus, 53) hind leg of $M$. vexator, 54) fore wing of $M$. ipidivorus, 55) dorsal view of the head of $M$. punctifrons, 56) fore wing of $M$. radialis. 

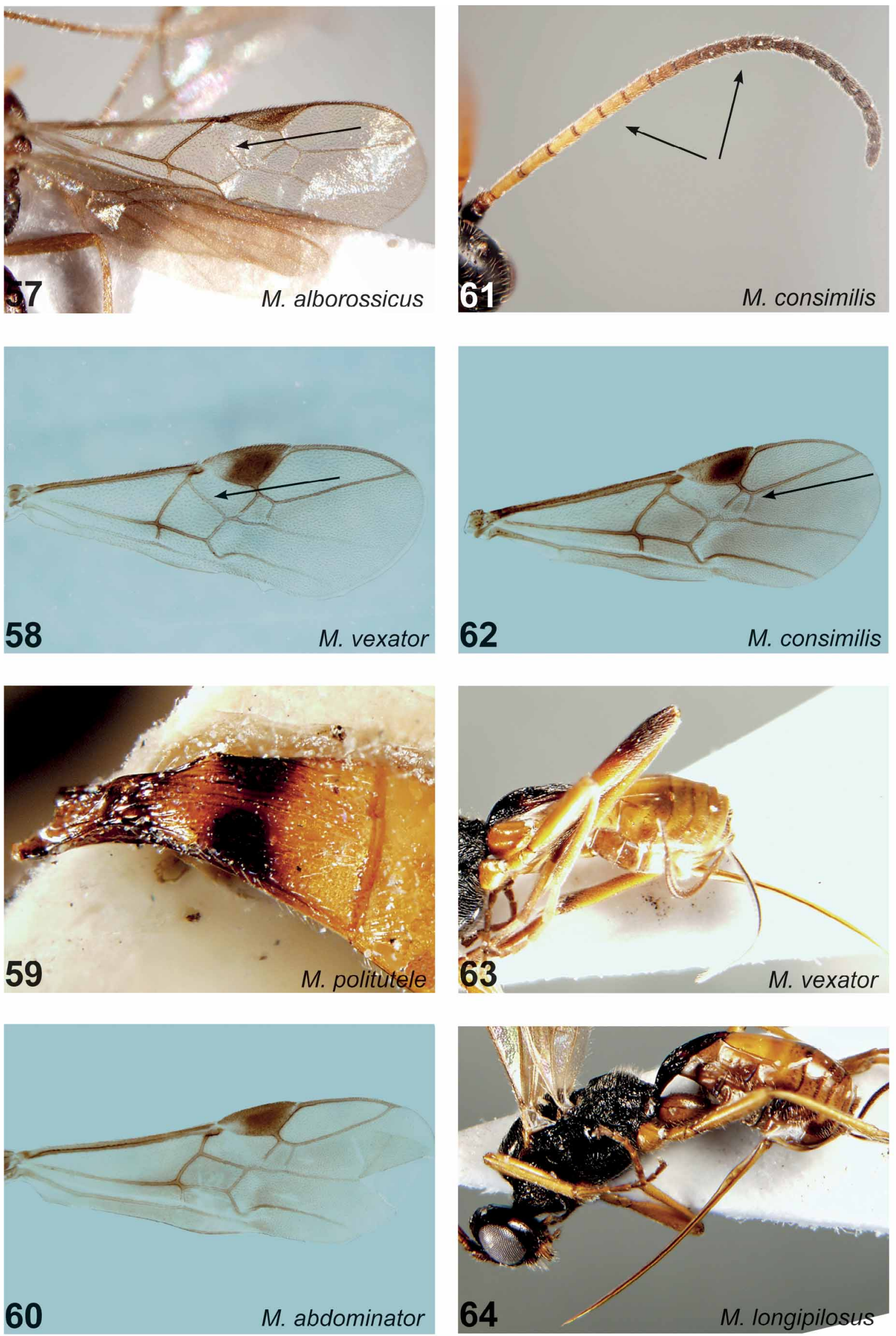

FIGURES 57-64. Characters used in key to species. 57) for wing of M. alborossicus, 58) for wing of M. vexator, 59) dorsal view of the petiolar tergum of $M$. politutele, 60) fore wing of M. abdominator, 61) antennae of M. consimilis, 62) fore wing of $M$. consimilis, 63) lateral view of the metasoma of $M$. vexator, 64) lateral view of mesosoma and metasoma of M. longipilosus. 

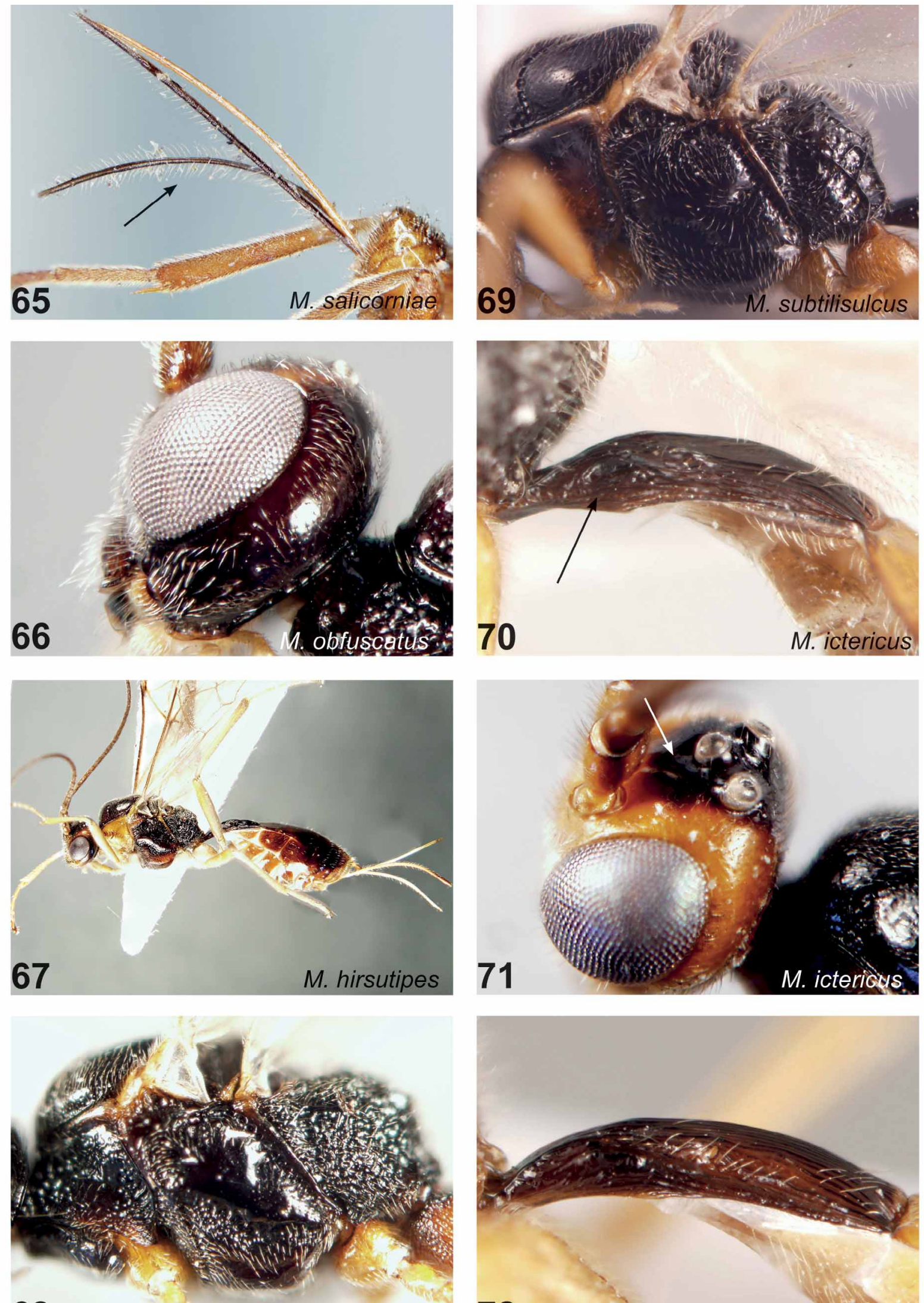

68

M. eklundi

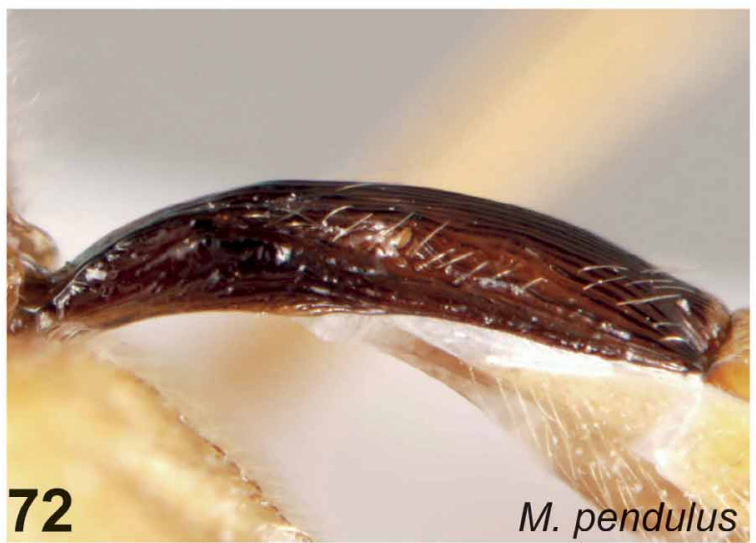

FIGURES 65-72. Characters used in key to species. 65) lateral view of metasomal part with ovipositor of $M$. salicorniae, 66) lateral view of the head of $M$. obfuscatus, 67) habitus of $M$. hirsutipes, 68) lateral view of mesosoma of $M$. eklundi sp.nov. 69) lateral view of mesosoma of M. subtilisulcus sp.nov., 70) lateral view of petiolar tergum of $M$. ictericus, 71 ) dorso-lateral view of the pit in front of the anterior ocelli of M. ictericus, 72) lateral view of petiolar tergum of M.pendulus. 

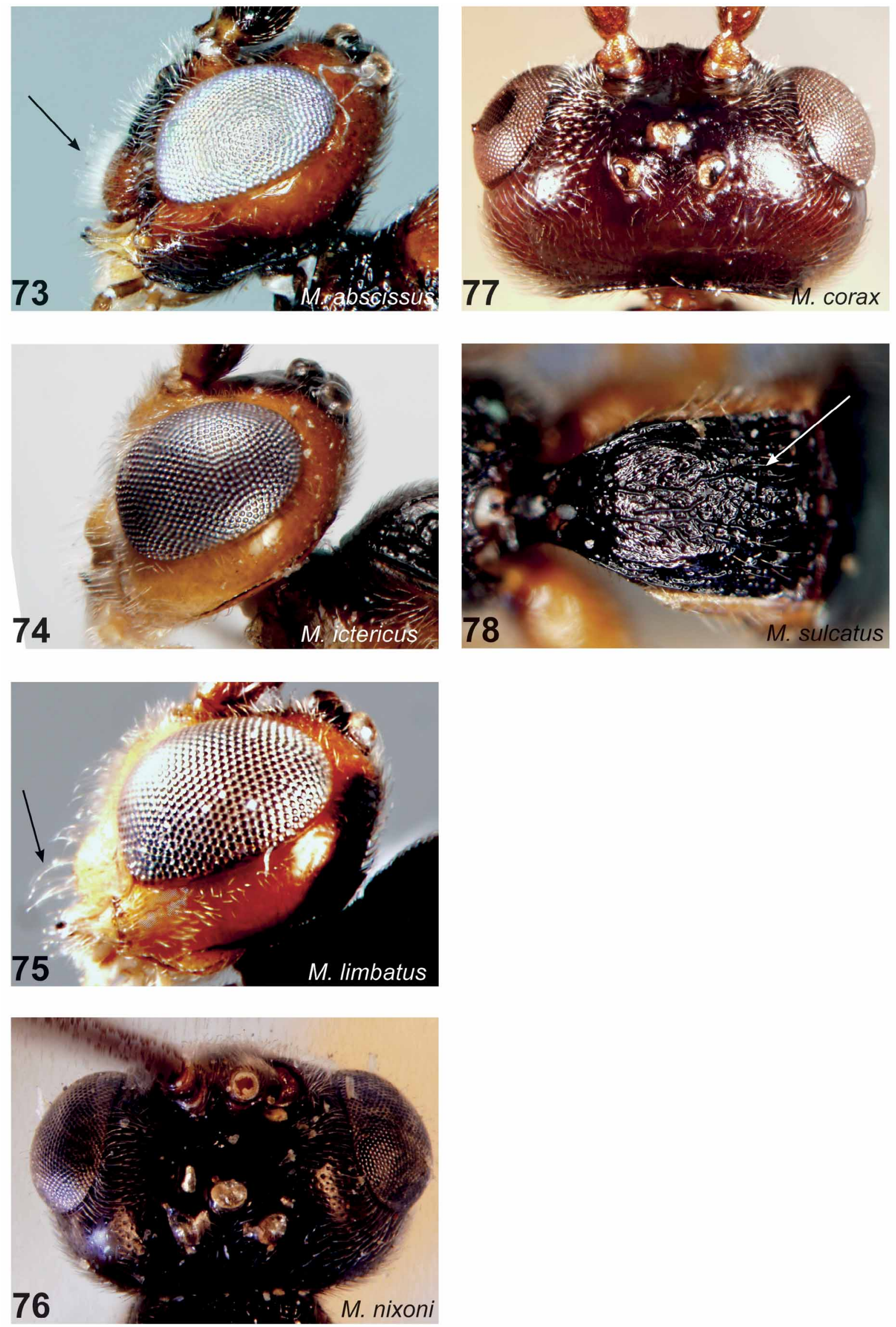

FIGURES 73-78. Characters used in key to species. 73) lateral view of the head of M. abscissus, 74) lateral view of the head of M. ictericus, 75) lateral view of the head of M. limbatus, 76) dorsal view of the head of M. nixoni, 76) dorsal view of the head of M. corax, 78) dorsal view of the petiolar tergum of M. sulcatus. 


\section{Genus Zele Curtis}

Zele Curtis 1832:415. Type species: Zele testaceator Curtis, 1832.

Zemiotes Foerster, 1862:253. Type species: Perilitus albiditarsus Nees, 1834. Synonymized by Van Achterberg 1979:376

Protelus Foerster, 1862:253. Type species: Perilitus chrysophthalamus Nees 1811.

Meteorus Haliday, 1835:24. Type species: Ichneumon pendulator Latreille 1799.

Diagnosis: The genus Zele is at a first glance most similar to Homolobinae. The larger and more common species (Z. albiditarsus, Z. chlorophthalmus and Z. deceptor), in particular, are similar in size and colour to Homolobinae, but the shape of the second abdominal (petiolar) tergum is a good diagnostic character: it is almost parallel and only slightly broadening in Homolobinae and widely broadening in Zele. The smaller species of Zele (Z. annulicrus and Z. caligatus) are more similar to species of the genus Meteorus, but are easily distinguished from the latter by the apically widened marginal cell of the hind wing (Fig. 12a), and the setose 3rd and following abdominal tergites. The marginal cell in Meteorus is apically narrowed and the abdominal tergites only have one row of setae each.

Distribution: Western and Eastern Palearctic, Nearctic, Neotropic, Oceanic, and Oriental regions.

Biology: The species of Zele mentioned here are all solitary except possibly Z.caligatus. As far as known, they all parasitize on Lepidoptera larvae. They are caught in Malaise traps from June to October. Some species are attracted to light.

\section{Zele albiditarsus Curtis, 1832}

Fig. 10a, 16, 134

Zele albiditarsus Curtis, 1832:415 Holotype đ̊ Great Britain: "Regents Park, England” (NMV, Melbourne).

Zele testaceator Curtis, 1832:415 Lectotype $\circ$, (NMV, Melbourne). Synonymized by Van Achterberg 1979:376

Perilitus albitarsus Nees von Eisenbeck, 1834:34

Meteorus albitarsis Haliday, 1835:24

Perilitus dispar Wesmael, 1835:22 Lectotype $q$, Belgium: Brussels, Wesmael coll. (IRSNB, Brussels).

Meteorus calcitrator Curtis, 1837:118 Synonymized by Van Achterberg 1979:376

Meteorus(Perilitus) wesmaeli Boie 1850:214 Lectotype ㅇ (ZM, Kiel).

Perilitus pallitarsis Cresson, 1872:81 Holotype ô,, (ANSP, Philadelphia). Synonymized by Van Achterberg 1979:376

Meteorus testatrix Schulz 1906: Spolia Hymenopterologica. Paderborn (Junfermann). 356pp.

Meteorus maximus Muesebeck 1923:13 Holotype ${ }_{+}$, (USNM Smithsonian Institute, Washington). Synonymized by Van Achterberg 1979:376

Diagnosis: Most similar to $Z$. deceptor, from which some specimens may be hard to separate. The variability in size and colour is very large in Z. albiditarsus but the short and wide fore femur (5.0-6.0 times as long as wide) and the long fore tibia spur (0.4-0.5 times fore basitarsus) are good characters to distinguish this species from $Z$. deceptor, which has a more slender fore femur (6.5-9.0 times as long as wide) and a shorter fore tibia spur (0.2-0.4 times fore basitarsus).

Studied Material: 200 specimens.

Description: Antennal articles 37-50. Ocelli large, $\mathrm{OOL}=1$. Eyes large, protuberant and not convergent. Malar space about 0.25 times mandible base. Face 1.4 times wider than high. Clypeus wide as face and slightly protuberant. Mandibles stout and twisted. Precoxal sulcus broad. Propodeum with distinct lateral and median longitudinal carinae. Length of petiolar tergum 2.5 times basal width; with dorsope. Laterope deep and joined dorsally with dorsope. Ventral borders of petiolar tergum not touching, widely separated by sternite. Ovipositor short and stout, 1.6 times as long as petiolar tergum. Hind coxa smooth punctate, tarsal claws lobed. Vein cu-a of fore wing postfurcal or interstitial, exceptionally antefurcal. Colour testaceous.

Distribution: Occurs throughout the Palearctic. Country records: Armenia; Austria; Azerbaijan; Belgium; Bulgaria; Canada; China; Croatia; Czechoslovakia; Denmark; Finland; France; Georgia; Germany; Greece; Hungary; India; Ireland; Israel; Italy; Japan; Kazakhstan; Korea; Latvia; Lithuania; Mexico; Moldova; Mongolia; Nepal; Netherlands; Norway; Poland; Romania; Russia; Slovakia; Sweden; Switzerland; Turkey; USA; Ukraine; United Kingdom; Yugoslavia. 
Biology: Zele albiditarsus is known to emerge from both the larval and pupal stage of the host. It is a solitary parasitoid that has been record as parasitizing on several Lepidoptera families: Geometridae, Noctuidae, Nymphalidae, Pyralidae, Saturnidae, Tortricidae and the Hymenoptera families Diprionidae and Ichneumonidae (Yu et al. 2005). We found 64 specimens in the SMTP material caught during the period June to October. Of these, 49 were caught in the same trap placed in a Hornbeam forest in the southern parts of Sweden.

\section{Zele annulicrus (Thomson, 1895)}

Fig. 135

Meteorus annulicrus Thomson, 1895:2161 Lectotype + , Sweden: (MZLU, Lund) - examined.

Diagnosis: Precoxal sulcus narrower and smaller than in other Zele species except Zele caligatus. However, the species is easily distinguished from the latter by the ovipositor being much longer ( 2.5 times petiolar tergum in $Z$. annulicus and 1.5 times petiolar tergum in Z. caligatus).

Studied Material: $\sim 20$ specimens.

Description: Antennal articles 31. OOL=1.3. Eyes large, protuberant and not convergent. Malar space 0.4 times basal width of mandibles. Face 1.5 times higher than broad. Clypeus protuberant. Mandibles stout. Precoxal sulcus narrow. Length of petiolar tergum 1.7 times its apical width, the surface distinctly rugulose. Ovipositor sheath 0.19 times fore wing. Hind coxa weakly punctate, tarsal claws with distinct lobe. Vein cu-a of fore wing postfurcal; pterostigma dark brown.

Distribution: Austria; Norway; Poland; $\underline{\text { Sweden. }}$

Biology: A rare species where the biology is unknown.

\section{Zele caligatus (Haliday, 1835)}

Fig. 14, 136

Meteorus caligatus Haliday, 1835:25 Lectotype $\odot$, Ireland: (NMI, Dublin).

Meteorus neesii Ruthe, 1862:22 Holotype o, Germany: (BMNH, London).

Dyscoletes alaskensis Ashmead, 1902:247 Alaska: (USMN, Washington). Synonymized by Van Achterberg 1979:364.

Meteorus sibiricus Fahringer, 1930:8, Type + , Russia: Kamtchatka (NHRS, Stockholm). Synonymized by Van Achterberg 1979:364-examined

Diagnosis: Similar to Z. annulicrus in the small size and narrow shape of the precoxal sulcus, but differs from that species in having a distinctly shorter ovipositor (2.5 times petiolar tergum in Z. annulicus and only 1.5 times petiolar tergum in Z. caligatus).

Studied Material: $\sim 50$ specimens.

Description: Antennal articles 35-37. OOL=1.5. Eyes large, protuberant and not convergent. Malar space 0.4 times basal width of mandibles. Face 1.5 times wider than high. Clypeus almost as wide as face, punctate and protuberant. Mandibles stout and twisted. Precoxal sulcus narrow. Length of petiolar tergum 1.7-1.8 times its apical width. Propodeum with a weak transverse carina. Ovipositor length 1.1-1.4 times petiolar tergum. Petiolar tergum with deep laterope joining dorsally with dorsope. Ventral borders of the petiolar tergum widely separated. Hind coxa weakly punctulate, tarsal claws lobed. Vein cu-a of fore wing postfurcal; the studied specimens were dark reddish brown.

Distribution: Occurs throughout the Palearcitc and Nearctic. Country records: China; Finland; Germany; Ireland; Italy Japan; Netherlands; Norway; Poland; Russia; $\underline{\text { Sweden; Switzerland; U.S.A.; United Kingdom. }}$

Biology: Zele caligatus is more frequent than Z. annulicrus but is still rather rare. It is a solitary parasitoid on Geometridae and Nymphalidae and emerges from the larval stage (Yu et al. 2005). Known hosts of the examined specimens all belong to the genus Eupithecia (Geometridae) (van Achterberg 1979, Stigenberg \& Shaw in prep.). One record of a gregarious rearing is mentioned in Hammond et al. (1957), where the material was determined by G. E. J. Nixon. The cocoon of Z. caligatus is whitish and the species is attracted to light and caught in Malaise traps. In the SMTP one specimen was caught in a Malaise trap in July in an old aspen forest. 


\section{Zele chlorophthalmus (Spinola, 1808)}

Fig. 137

Bracon chlorophthalmus Spinola, 1808:133 Neotype $\odot$ (RMNH, Leiden)

Bracon chrysophthalmus Nees von Esenbeck, 1811:21 Neotype 우, Wesmael coll. (IRSNB, Brussels). Synonymized by Van Achterberg 1979:370.

Bracon pallidus Nees von Esenbeck, 1811:22 Type material lost. Syn. n.

Ichneumon nudator Thunberg, 1822:263 Holotype $\widehat{\delta}$, Thunberg coll. (UDE, Uppsala). Synonymized by Van Achterberg 1979:364.

Meteorus splendens Costa, 1884:171 Holotype ${ }_{+}$, Italy: Sardinia. A. de Costa Lima coll. (USN, Naples). Synonymized by Van Achterberg 1979:364.

Meteorus (Zemiotes) nigricollis Thomson, 1895:2150 Holotype ${ }_{+}$, Sweden, Degerberga (MZLU, Lund). Synonymized by Van Achterberg 1979:364

Diagnosis: Zele chlorophthalmus has a slightly darker and more slender appearance than the two other "larger" Zele species: Z. deceptor and Z. albiditarsus. The best way to separate Z. chlorophthalmus from the other two is to study the wing vein cu-a of the fore wing. This vein is antefurcal in Z. chlorophthalmus and postfurcal in Z. deceptor and Z. albiditarsus. Also, the ovipositor is longer (2.6 times petiolar tergum) in Z. chlorophthalmus than in Z. albiditarsus and $Z$. deceptor (1.6 times petiolar tergum in both species).

Studied material: $\sim 50$ specimens.

Description: Antennal articles 36-42. OOL=1.2. Eyes large, slightly protuberant and not convergent. Malar space 0.1 times basal width of mandibles, eyes almost touching mandibles. Face 1.3 times wider than high. Clypeus almost as wide as face, protuberant and punctate. Mandibles stout and twisted. Precoxal sulcus wide. Length of petiolar tergite 2.1-2.4 times its apical width. Propodeum with a median carinae. Ovipositor long, 2.6 times petiolar tergum. The petiolar tergum with deep laterope joining dorsally with dorsope. Ventral borders widely separated. Hind coxa punctulate, tarsal claws lobed. Vein cu-a of fore wing antefurcal or interstitial. Large, and body colour variable, varies from yellow or brown to mainly dark brown or blackish.

Distribution: Eastern and Western Palearctic; Madagascar and India. Country records: Norway; Sweden; United Kingdom.

Biology: Zele chlorophthalmus is a solitary parasitoid recorded as parasitizing on the larval stages of eight different Lepidoptera families: Depressariidae, Geometridae, Lasiocampidae, Lymantriidae, Noctuidae, Pyralidae, Tortricidae and Zygaenidae (Yu et al. 2005). It has been used as a biocontrol agent for Ostrina nubilalis (Lepidoptera). We found 9 specimens within the SMTP. They were caught from June to August in deciduous forest or in close vicinity of meadows.

\section{Zele deceptor (Wesmael, 1835)}

Fig. 11, 15, 17, 138

Perilitus deceptor Wesmael, 1835:26 Lectotype ${ }_{+}$, Belgium: Brussels, Wesmael coll. (IRSNB, Brussels). Synonymized by Van Achterberg 1979:376 and stat. nov as valid species 1984:110

Perilitus pallitarsis Cresson, 1872:81 Holotype $\hat{\jmath}$, (ANSP, Philadelphia). Synonymized by Van Achterberg 1984:110

Meteorus (Zemiotes) rufulus Thomson, 1895:2149 Lectotype ô, Sweden: (ZML, Lund). Synonymized by Van Achterberg 1984:110

Meteorus maximus Muesebeck 1923:13 Holotype ${ }_{+}$, (USNM, Smithsonian Institute, Washington). Synonym for rufulus. Synonymized by Van Achterberg 1984:110

Meteorus reticulatus Muesebeck 1923:14 Type (USNM, Washington). Synonymized by Van Achterberg 1984:110

Meteorus (Zemiotes) romani Fahringer, 1930:8 Holotype + , (NHRS, Stockholm). Synonymized with M. rufulus by Van Achterberg 1984:110 - examined

Meteorus separandus Fischer, 1957:3 Paralectotype ô, (NHRS, Stockholm). Synonymized by Van Achterberg 1984:110examined

Diagnosis: In general, Zele deceptor is similar to the other large Zele species, Z. chlorophthalmus and Z. albiditarsus. It can be distinguished from the former based on wing venation and ovipositor characters, as described under that species, but it can be difficult to distinguish from Z. albiditarsus because of the large variability in size and 
colour. However, the slender fore femur (slender, 6.5-9.0 times as long as wide) and the short fore tibia spur (0.20.4 times fore basitarsus) are good characters to distinguish Z. deceptor from Z. albiditarsus, which has a stouter fore femur (5.0-6.0 times as long as wide) and a longer fore tibia spur (0.4-0.5 times fore basitarsus).

Studied material: $\sim 200$ specimens.

Description: Antennal articles 32-43. OOL=1. Eyes large, protuberant and not convergent. Malar space 0.3 times basal width of mandibles. Face 1.1-1.3 times wider than high. Clypeus protuberant almost as wide as face. Mandibles stout and twisted. Precoxal sulcus wide. Length of petiolar tergum 2.1-2.5 times its apical width. Propodeum with weak median and lateral longitudinal carina, dorsal transverse carina weak. Ovipositor stout, 1.6 times petiolar tergum, slightly longer than half of abdomen. Petiolar tergum with smooth laterope joining dorsally with dorsope, its ventral borders widely separated. Hind coxa smooth, tarsal claws lobed. Vein cu-a of fore wing postfurcal, seldom interstitial; Colour mostly testaceous but variation in size and colour is very large in M. deceptor.

Distribution: Palearctic and Nearctic. Country records: Albania; Austria; Belgium; Bulgaria; Canada; China; Croatia; Czechoslovakia; Denmark; Finland; France; Georgia; Germany; Hungary; Ireland; Italy; Japan; Kazakhstan; Latvia; Lithuania; Mexico; Netherlands; Norway; Poland; Romania; Russia; Slovakia; Sweden; Switzerland; USA; United Kingdom; Yugoslavia.

Biology: Zele deceptor seems to be as frequent as Z. albiditarsus in museum collections. It is a solitary parasitoid, also attracted to light, and emerges from the larval stages of various lepidopteran families, such as Geometridae, Noctuidae, Pyralidae and Saturnidae (Yu et al. 2005). 19 specimens of Z. deceptor were caught in SMTP from July to October in various biotopes. A trap situated in grazed calcareous pine forest collected most specimens (5).

\section{Genus Meteorus Haliday}

Meteorus Haliday, 1835, (as subgenus of Perilitus); Type species Ichneumon pendulator Latreille, 1799, designated by Haliday in Westwood, 1840.

Saprostichus Holmgren 1868, Type species: Saprostichus chinensis Holmgren 1868, designated by Viereck 1914.

Pachythechus Cameron, 1912:84; Type species: Pachythechus ruficeps Cameron 1912, designated by Viereck, 1914. (Meteorus cameroni Shenefelt, 1969, replacement name for ruficeps Cameron 1912, preoccupied by M.ruficeps Nees, 1834).

Diagnosis: The species of Meteorus vary greatly in size from 2-10 mm. They are easily distinguished from other braconids by the petiolate second abdominal tergum in combination with the second submarginal cell in the fore wing and the presence of only one row of setae (dorsally) on each abdominal tergite from the $3^{\text {rd }}$ and onwards. The larger species might at first glance be mistaken for members of Zele but are distinguished from the latter by the single row of setae on the adominal tergites.

Distribution: Western and Eastern Palearctic, Nearctic, Neotropic, African, Oceanic and Oriental regions.

Biology: Meteorus contains both solitary and gregarious species. They parasitize on both Lepidoptera and Coleoptera. Some species have been used in biological control programmes (Huddleston 1980, Yu et al. 2005).

\section{Meteorus abdominator (Nees von Esenbeck)}

Fig. 60, 80

Perilitus abdominator Nees von Esenbeck, 1811:24. Syntypes $\widehat{o}$ and $\odot$, Germany destroyed. Perilitus brunnipes Ruthe, 1862:37. Lectotype ${ }_{+}$, Germany: Ruthe coll. (BMNH, London) examined. Meteorus brevipesalis Shenefelt, 1969:55. Holotype , Belgium: Wesmael coll. (IRSNB, Brussels)

Diagnosis: Meteorus abdominator is similar to M. consimilis and M. longipilosus but the size of ocelli and length of ovipositor separate them. The OOL in $M$ abdominator is 3.5-4.0 and the ovipositor length 2.0-2.5 times the length of the petiolar tergum. In M. consimilis the OOL is 1.5 and the ovipositor is short, thick and downcurved; at most equal in length to the petiolar tergum. M. longipilosus is very similar to M. abdominator but has an OOL of 2.5 and an ovipositor length of 3.1 times the length of the petiolar tergum.

Studied material: $\sim 100$ specimens. 
Description: Size about 4-5mm. Antennal articles 22-25; flagellum thick and at apex as broad as long, in some specimens the basal flagellar articles is lighter in colour. Head and face distinctly transverse, about twice as broad as high. Ocelli small, $\mathrm{OOL}=3.5-4.0$. Eyes not so large, converging slightly. Malar space slightly shorter than basal breadth of mandible. Tentorial pits deep. Clypeus strongly protuberant. Mandible stout, twisted. Precoxal sulcus broad. Propodeum reticulate-rugose and carinae distinct, sometimes obscured. Petiolar tergum stout, with distinct dorsal pits. Ovipositor 2.0-2.5 times length of petiolar tergum and down-curved. Legs stout, hind coxae dorsally punctuate to rugose, tarsal claws not strongly bent but swollen at base. Wings short, infumate; second submarginal cell of fore wing more or less strongly contracted anteriorly. Colour dark - black; Male antennae longer, 25-31 articles.

Distribution: Palearctic. Country records: Armenia; Azerbaijan; Belgium; Bosnia Hercegovina; Bulgaria; Croatia; Czechoslovakia; Denmark; Finland; France; Germany; Hungary; Ireland; Italy; Kazakhstan; Korea; Latvia; Lithuania; Moldova; Netherlands; Norway; Poland; Romania; Russia; Sweden; Switzerland; Ukraine; United Kingdom; Yugoslavia.

Biology: Parasitizes on Lepidoptera, mostly Geometridae (Huddleston 1980, Yu et al. 2005). We found 48 specimens within the SMTP and they were caught from June to November. Based on these records, M. abdominator seems to prefer deciduous forests.

\section{Meteorus abscissus Thomson}

Fig. 73, 81

Meteorus abscissus Thomson, 1895:2156. Lectotype + , Sweden: (MZLU, Lund)—examined.

Diagnosis: Meteorus abscissus is close to M. pulchricornis. They both share the character of having short dense setae on the clypeus. However, M. abscissus has a more stout body shape and its clypeus is more flat and the sculpture of hind coxae and mesosoma is more strongly rugose. Furthermore, M. abscissus has the ventral sides of the petiolar tergum separated, contrary to M. pulchricornis, which has the ventral sides of the petiolar tergum closed. Finally, M. abscissus has a true dorsope even though it can sometimes be so small that it is difficult to separate from the small indication of a dorsope present in M.pulchrichornis.

Studied material: $\sim 20$ specimens.

Description: Size about 4-5mm. Antennal articles 30-33, all flagellar articles distinctly long. Head broad, temples strongly contracted behind eyes. Ocelli large $\mathrm{OOL}=2$. Eyes large, moderately convergent. Malar space about equal to basal breadth of mandible. Face 1.5 times as wide as high, not strongly protuberant. Clypeus peculiarly flattened, slightly protuberant. Mandibles slender and twisted. Precoxal sulcus broad. Propodeum broad, strongly convex and reticulate-rugose. Petiolar tergum long with distinct dorsal pits and shallow glymmae. Ovipositor 1.5-2.0 times length of petiolar tergum, straight. Hind coxa strongly rugose. Tarsal claws with a distinct basal lobe. Colour on holotype is dark read with black markings but some specimens are brownish testaceous. 34 34 36 antennal articles; petiolar tergum narrower; sculpture of hind coxa sometimes obsolescent.

Distribution: Palearctic. Country records: Albania; Austria; Bulgaria; China; China-Fujian; Croatia; Czechoslovakia; Germany; Hungary; Ireland; Italy; Korea; Norway; Romania; Russia; Slovakia; Slovenia; $\underline{\text { Spain; }}$ Sweden; Switzerland; United Kingdom; Yugoslavia.

Biology: Meteorus abscissus is recorded as a parasitoid of several Lepidoptera families, in particular Arctiidae, Geometridae and Noctuidae (Yu et al. 2005). Old host records might be erroneous due to the difficulties in properly separating some intermediate specimens from M. pulchricornis. We found 4 specimens within the SMTP. They were caught in late July and early August in arid, exposed environments.

\section{Meteorus acerbiavorus Belokobylskij, Stigenberg and Vikberg}

Fig. 31, 82

Meteorus acerbiavorus Belokobylskij et al., 2011. Holotype ㅇ, "Finland 767:325. Enontekiö Lapland: Jehkats, June 2009, Harry Lonka leg". 
Diagnosis: A very distinctive species, large, dark and rugose. The colour and sculpture can vary between localities and rearings from black to brown and from heavily to more weakly rugose, almost smooth in certain parts. The petiolar tergum also varies from specimens without a dorsope to specimens with a distinct dorsope. M. acerbiavorus is most similar to M. rubens, being easily separated by colour (completely black or brownish in the former, varying from completely reddish to partially, but never completely, black in the latter), body sculpture (largely rugulosegranulate in the former, smoother with only certain parts rugose in the latter), and the shape of the ovipositor tip (with a distinct dorsal node at the tip of the ovipositor in the former, without a distinct node in the latter) (illustrated in Stigenberg et al. 2011).

Studied material: 13 specimens.

Description: Size 4.3-4.6 mm. Antennal articles 25-27. Head transverse. OOL=1.5. Eyes large and protuberant, weakly convergent. Malar space equal to width of mandible base. Face 1.3 times wider than high, rugose. Clypeus almost as wide as face and protuberant. Mandibles twisted. Precoxal sulcus wide and entirely coarsely rugose-reticulate. Propodeum roundly convex, strongly reticulate. Petiolar tergum with no dorsope, finely striate at apical end. Length of petiolar tergum about twice as long as apically broad. Ovipositor 1.2-1.4 times longer than petiolar tergum. Legs long; hind coxae entirely rugose; tarsal claws without lobe. Colour black. $\hat{o}$ antennal articles $24-27$.

\section{Distribution: Finland.}

Biology: Meteorus acerbiavorus is only known from specimens reared from cocoons of the arctiid moth Acerbia alpina collected in June in nothern Finland (Stigenberg et al. 2011). It is gregarious and 39 to 98 parasitoid cocoons have been found in a single host cocoon.

\section{Meteorus affinis (Wesmael)}

Fig. 83

Perilitus affinis Wesmael, 1835:31. Holotype ${ }_{+}$, Belgium: Wesmael coll. (IRSNB Brussells) examined.

Meteorus punctiventris Ruthe, 1862:25. Holotype 9 , Germany: Ruthe coll. (BMNH London) examined

Meteorus gracilis Ruthe, 1862:31. Holotype , Germany: Ruthe coll. (BMNH London). Junior primary homonym of gracilis Ratzeburg, 1852.

Meteorus ruthei Schmiedeknecht, 1897:205. Replacement name for gracilis Ruthe, 1862. Synonymized by Huddleston 1980:22.

Meteorus voloscensis Fischer, 1959:14 Holotype $q$ Yugoslavia: Volosca, Graeffe coll. (NM, Vienna) Synonymized by Huddleston, 1980:22 examined.

Diagnosis: Meteorus affinis is similar to M. vexator but has distinctly lobed claws and larger ocelli than the latter. These differences are visible also in small specimens, which are otherwise very similar due to their weaker sculpture and the reduction in the number of antennal articles. The mesosoma of M. affinis sometimes has yellow markings, but we never found this to be true for M. vexator.

Studied material: $\sim 100$ specimens.

Description: Size about $4 \mathrm{~mm}$. Antennal articles 27-30. Head strongly rounded behind eyes. Ocelli large, $\mathrm{OOL}=1.5-2.0$. Eyes large, protuberant and strongly convergent. Malar space short, much less than basal width of mandible. Face as broad as high, not protuberant but rugulose. Clypeus not strongly protuberant. Mandible stout, moderately twisted. Precoxal sulcus foveolate. Propodeum rugose, the transverse carinae of the propodeum often most developed. Petiolar tergum narrow with distinct dorsal pits, about twice as long as apically broad. Ovipositor long, 3.0-3.5 times length of petiolar tergum, slender. Legs long, slender; hind coxae rugose ventrolaterally; tarsal claws with small but distinct lobe. Colour generally black with yellow areas. $\hat{o}$ antennal articles 30-33; eyes smaller and not strongly convergent; sculpture of precoxal sulcus and of hind coxa obsolescent.

Distribution: Western and Eastern Palearctic. Country records: Armenia; Belgium; Bulgaria; China; Croatia; Czechoslovakia; Finland; France; Germany; Hungary; Ireland; Italy; Korea; Netherlands; Norway; Romania; Russia; $\underline{\text { Sweden; }}$ Switzerland; United Kingdom; Yugoslavia.

Biology: Meteorus affinis is a solitary endoparasitoid that is attracted to light. Most host records involve the lepidopteran family Psychidae (Yu et al. 2005). We found 59 specimens within the SMTP. M. affinis was caught from June to November in both deciduous and coniferus forests. 


\section{Meteorus alborossicus Lobodenko}

Fig. 57, 84

Meteorus alborossicus Lobodenko, 2000. Holotype ${ }_{+}$, Russia: 'Byelorussia, Beresina Nat. Res., Postrezhie, 1vii1.viii.1996’ (Lobodenko) (ZIN. St. Petersburg) - examined.

Diagnosis: Meteorus alborossicus is close to $M$. vexator but the fore wing venation easily distinguishes them: while $M$. alborossicus has the fore wing vein 1-SR+M incomplete, it is complete in M.vexator. M. alborossicus is actually the only species of Meteorus in Europe with an incomplete 1-SR+M vein.

Studied material: $\sim 20$ specimens.

Description: Size about 3-4mm. Antennal articles 29. Ocelli small, $O O L=2-2.5$. Eyes protuberant, large and converging. Malar space narrow, 0.4 times basal width of mandibles. Face as wide as high and punctuate, clypeus also punctate. Clypeus protruding and variable in width, sometimes as wide as face. Mandibles only slightly twisted. Precoxal sulcus wide. Propodeum with transverse carinae. Petiolar tergum rugose centrally, striate laterally. Ovipositor long and slender, more than 2.5 times petiolar tergum. Fore and middle coxa light coloured (whitish), hind coxa dark and rugose. Fore wing vein $1-\mathrm{SR}+\mathrm{M}$ not complete. Claws slender but with a slight widening at base.

Distribution: Western Palearctic. Country records: Belarus; Sweden; United Kingdom.

Biology: We found a single specimen within the SMTP; it was caught on a sandy railway in August. Three specimens were reared in an ongoing study on saproxylic insects on wet stumps at the Swedish University of Agricultural Sciences (Ols in prep.)

\section{Meteorus angiclypealis van Achterberg}

Fig. 85

Meteorus angiclypealis, 2001:397. Holotype ${ }_{+}$, Netherlands: (RMNH, Leiden)-examined

Diagnosis: This species is close to M. sibyllae but is distinguished by two good characters: (1) the shape of the head, which is transverse in M. sibyllae and rounded in M. angiclypealis; and (2) the length of the ovipositor, which is 3.5-4.0 times petiolar tergum in M. sibyllae and 2.7 times petiolar tergum in M. angiclypealis.

Studied material: One specimen, the holotype.

Description: Size about $3 \mathrm{~mm}$. Antennal articles 24, all articles longer than broad. Head rounded behind eyes. $\mathrm{OOL}=3.5$. Eyes small, not converging. Length of malar space 0.6 times basal width of mandible; mandibles hardly or not twisted apically. Face punctulate, dorsally with some rugae. Clypeus protruding, punctuate and with some long setae, width of clypeus 0.7 times minimum width of face. Precoxal sulcus narrow. Propodeum with transverse carina, without median carina. Surface of propodeum largely smooth except for subbasal transverse carina. Length of petiolar tergum 1.6 times its apical width, surface punctate medially and laterally striated. Dorsope distinct but rather small. Length of ovipositor sheath 0.54 times fore wing and 2.7 times petiolar tergum. Hind coxa largely smooth, claws without lobe. Fore wing m-cu antefurcal. Pterostigma dark brown, basally with small pale spot.

Distribution: $\underline{\text { Switzerland. }}$

Biology: The holotype of M. angiclypealis was caught in a light trap.

\section{Meteorus artocercus Stigenberg sp. nov.}

Fig. 48, 49, 79A, 86

Diagnosis: Meteorus artocercus can be distinguished from similar species by its longer flagellomeres, the colour of the pterostigma and the length of the ovipositor sheaths. In Huddleston's key M. artocercus runs to M. cinctellus but $M$. artocercus has longer antennal articles than M. cinctellus, the ovipositor is shorter (1.6-2.0 times petiolar tergum in the former, and 2.5-3.0 times in the latter), and the pterostigma is pale (dark brown except for the basal third in the latter). M. tenellus is another similar species but this species has a longer ovipositor (2.0-4.0 times petiolar tergum) and a postfurcal m-cu vein of the forewing (antefurcal in M. artocercus). 
Studied material: 10 specimens.

Holotype: 1 \& SE. Lapl. Gällivare kommun. Ätnarova försökpark, Pelttovaara. 29.vii-13.viii.2004. (leg. SMTP), NHRS-HYME 5178. Paratypes: 2 SE. Lapl. Gällivare kommun. Ätnarova förökspark, Pelttovaara. 29.vii-13.viii.2004. One in ethanol (DNA voucher specimen DNA 1) and one drypinned (one leg damaged and glued separately on the dry pinned specimen) NHRS-HYME 5176 (leg. SMTP), Paratype: 1 \& SE. Nb. Pajala kommun. Vasikkavuoma. 14.ix-05.x.2003. (left hind tarsi damaged and glued separately). (leg. SMTP), NHRS-

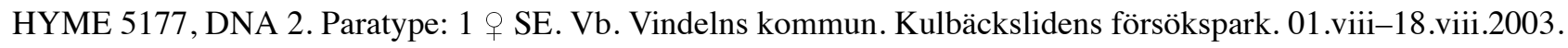
Kept in $80 \%$ ethanol. (leg. SMTP), DNA 47. Paratype: 4 \% SE. Bdn. Jokkmokks kommun, Muddus nationalpark 18.vi-20.vii.2004. All are kept in $80 \%$ ethanol and all are DNA voucher specimens (leg. SMTP), NHRS-HYME 5182, DNA 55; NHRS-HYME 5181, DNA 56; NHRS-HYME 5180, DNA 57; NHRS-HYME 5179, DNA 58.

Description: Body $3.2 \mathrm{~mm}$, fore wing $3.0 \mathrm{~mm}$.

Head: Length of eye 1.5 times length of temple in dorsal aspect; eyes large, convergent and protuberant; width of face 1.25 times its height; $\mathrm{OOL}=2$; vertex smooth; frons above antennal sockets depressed, with a median ridge forming a slight tubercle in front of fore ocellus; face convex; clypeus as wide as minimum width of face, distinctly separated from face, convex, tentorial pits distinct; length of malar space 0.6 times basal width of mandible; mandibles twisted; antennal articles 27; 3rd and 4th antennal article 3.6 times longer than wide; all articles longer than wide, subapical articles more than 1.5 times longer than broad.

Mesosoma: Precoxal sulcus narrow.

Wings: Fore wing: $\mathrm{r} / 3-\mathrm{SR}=0.6 ; \mathrm{C}+\mathrm{SC}+\mathrm{R} / \mathrm{SR} 1=1.45 ; \mathrm{m}-\mathrm{cu}$ interstitial; pterostigma brown with apical corner light. Hind wing: $1-\mathrm{M} / \mathrm{cu}-\mathrm{a}=1.5$;

Legs: Hind coxa rugose dorsally; hind femur 4.8 times longer than wide; tarsal claws small with lobe.

Metasoma: Petiolar tergum apically 2.1 times longer than wide, petiolar tergum 4.5 times fore wing; dorsope absent; ventral folds of petiolar tergum joined in middle; ovipositor straight, slender; length of ovipositor 2.0 times that of petiolar tergum and 0.35 times forewing; propodeum convex, reticulate-rugose and with no distinct carina. Colour: Frons and vertex black with large yellow patch between posterior ocelli and eye; face yellow; pronotum and mesopleuron black with brown-red patch posterior; petiolar tergum black; $3^{\text {rd }}$ abdominal tergite yellow otherwise brown; legs dark yellow, hind tarsi brown.

Distribution: Sweden.

Biology: We found 10 specimens within the SMTP. They were caught from July to October in northern Sweden, predominantly in pine forests.

Etymology: The name artocercus is derived from the Latin word arto-close and the Greek word cercustail.

\section{Meteorus breviantennatus Tobias}

Fig. 87

Meteorus breviantennatus Tobias, 1986:345. Holotype ${ }_{+}$, Russia: (ZIN. St. Petersburg)—examined.

Diagnosis: Meteorus breviantennatus is similar to $M$. cis but the clypeus of $M$. breviantennatus is almost as wide as the face while the clypeus of $M$. cis is narrower. Also the malar space (shorter than mandibular base on M. breviantennatus, as wide as mandibular base on M. cis) and the face (slightly transverse in M. breviantennatus, round in $M$. cis) should separate these species.

Studied material: 11 specimens.

Description: Size about 3mm. Antennae 20-22 articles, the basal articles lighter in colour. Head slightly transverse. Ocelli small, $\mathrm{OOL}=3-3.5$. Eyes small, slightly protuberant, convergent. Malar space shorter than basal breadth of mandible. Face about 1.3 times as broad as high, punctate, not strongly protuberant. Clypeus almost as wide as face, protruding, in some specimen with a notch similar to M. cis. Tentorial pits distinct. Mandibles short and moderately twisted. Precoxal sulcus rather wide. Propodeum with carinae, otherwise reticulate. Dorsal pits of the petiolar tergum small, dorsal surface rugose with longitudinal striae laterally. Ovipositor about twice the length of petiolar tergum. Legs slender; hind coxa punctuate with small punctures. Tarsal claws long and slender. Colour dark brown; clypeus, mandibles and usually third abdominal tergum of lighter colour than the rest of body; coxa and legs brownish-yellow. 
Distribution: Western and Eastern Palearctic. Country records: Austria; China; France; Germany; Ireland; Japan; Korea; Netherlands; Russia; Sweden; United Kingdom.

Biology: According to Tobias (1986) it is a parasitoid of Ips acuminatus (Gyllenhal, 1827) and Tomicus minor (Hartig, 1834) (Coleoptera).

\section{Meteorus brevicauda Thomson}

Fig. 88

Meteorus brevicauda Thomson, 1895:2165. Lectotype 9 , Sweden (MZLU, Lund - examined)

Meteorus thuringiacus Schmiedeknecht, 1897:190. Syntypes + , Germany (lost). Synonymized by Huddleston 1980:23.

Meteorus mongolicus Fahringer, 1935:10. Holotype , China: S. Kansu (Hummel) (NHRS Stockholm)—examined. Synonymized by Huddleston 1980:23.

Diagnosis: Meteorus brevicauda is a very small species and is easily distinguished amongst the other small species based on the shape of the clypeus (as wide as the face) and mandibles (large, stout and not twisted). M. micropilosus have a small, square clypeus and slightly twisted but stout mandibles. M. punctifrons is distinguished by the presence of punctures on the frons, which lack in M. brevicauda. M. breviantennatus have a wide clypeus similar to that of $M$. brevicauda, but it can easily be distinguished from the latter by having a longer ovipositor (twice the length of petiolar tergum in the former, 1.5 times petiolar tergum in the latter).

Studied material: $\sim 10$ specimens.

Description: Size about 3mm. Antennal articles 24-27, articles slightly longer than broad. Ocelli small, $\mathrm{OOL}=3$. Eyes not strongly protuberant, slightly converging. Malar space very short. Face rather flat and about 2 times as broad as high. Clypeus large, flat and as wide as face. Tentorial pits large. Mandibles large, stout and not twisted. Precoxal sulcus foveolate. Propodeum short with distinct carinae. Petiolar tergum with distinct dorsal pits and glymmae. Ovipositor short, about 1.5 times the length of petiolar tergum. Legs short, the hind coxa smooth, punctuate; tarsal claws with no basal lobe but slightly swollen at the base. Colour generally black except antennae at base, clypeus, mandibles and prothorax ventrally testaceous, legs yellow. Fore wing vein m-cu antefurcal.

Distribution: Palearctic. Country records: Austria; Bulgaria; China; Finland; Germany; Hungary; Italy; Lithuania; Poland; Sweden; Switzerland; United Kingdom.

Biology: Meteorus brevicauda parasitizes on the leaf-feeding larvae of Zeugophora subspinosa (Fabricius) (Coleoptera, Chrysomelidae) (Shaw, 1988).

\section{Meteorus cespitator (Thunberg)}

Fig. 89

Ichneumon cespitator Thunberg, 1822:269. Holotype $\odot$, Sweden (UDE, Uppsala) - examined.

Zele atrator Curtis, 1832: folio 415. Syntypes, Great Britain: Wilts, 'Durnford House' (NMV, Melbourne) Synonymised by Roman, 1912:289.

Perilitus similator Nees von Esenbeck, 1834:41 Syntypes ${ }_{+}$, Germany (destroyed). Synonymised by Haliday, 1835:32

Bracon humeralis Zetterstedt, 1838:399. Lectotype , Lapponia: 'Öfv. Tor.' (Övertorneå in Sweden). "mihi tantum inter Muonioniska et Kengis". Synonymised by Papp, 1994:305

Bracon rufipes Zetterstedt, 1838:399. Lectotype क, 'Johs. Ro.'(Johannisro, Norrbotten). "Hab. cum priori rarius" (B. humeralis). Synonymised by Papp, 1994:305

Meteorus ambiguus Ruthe, 1862:30. Holotype , , Germany: Ruthe coll., 4.10.55 (BMNH, London) examined.

Diagnosis: The size, the transverse head and the long ovipositor are characters that distinguish this species. Meteorus cespitator mostly resembles $M$. obfuscatus and the new species $M$. eklundi and $M$. densipilosus described in this paper. However, the long ovipositor of M. cespitator (3-4 times length of the petiolar tergum) clearly separates it from these species, which have ovipositors that are at most up to twice the length of the petiolar tergum.

Studied material: $\sim 50$ specimens.

Description: Size about 4-5mm. Antennal articles 23-27. Head transverse, temples shorter than eyes. Ocelli small, $\mathrm{OOL}=2.5-3.0$. Eyes large, protuberant, convergent but not strongly so. Malar space distinctly shorter than 
basal breadth of mandible. Face about 1.5 times as broad as high. Clypeus only slightly protuberant, distinctly divided from face. Precoxal sulcus deep. Propodeum irregularly rugose with distinct carinae. Ovipositor long, 3-4 times length of petiolar tergum. Hind coxae rugose, tarsal claws long, slightly swollen at base but not lobed. Colour generally dark - black with yellow parts.

Distribution: Palearctic and Oceania. Country records: Algeria; Azerbaijan; Belgium; Bulgaria; Canary Islands; China; Denmark; Faeroe Islands; Finland; France; Georgia; Germany; Hungary; Iceland; Ireland; Italy; Japan; Latvia; Lithuania; Luxembourg; Netherlands; New Zealand; Norway; Poland; Romania; Russia; Sweden; Switzerland; Turkey; United Kingdom.

Biology: Meteorus cespitator was accidentally introduced to New Zealand and there it was found to parasitize on Trichophaga tapetiella from ply-wood (Parrot 1955). M. cespitator parasitizes on Tineidae (Lepidoptera) (Hinton 1956) and it is attracted to light (Yu et al. 2005). We found 21 specimens within the SMTP; they were caught in deciduous or spruce forests from the end of June to the beginning of September.

\section{Meteorus cinctellus (Spinola)}

Fig. 42, 44, 90

Bracon cinctellus Spinola, 1808:135, Holotype $\widehat{\partial}$, Italy (MZS, Turin).

Bracon necator Trentepohl, Isis 1829 p 958 n. 75 , [excl. Synon.] of Bracon cinctellus Nees 1811

Perilitus fuscipes Wesmael, 1835:48, Holotype $\odot$, Belgium: Brussels, coll. Wesmael (IRSNB, Brussels) synonymized by Huddleston, 1980:25 - examined.

Meteorus tenellus Marshall, 1887:125. Lectotype $\odot$, Great Britain (BMNH, London), synonymized by Huddleston, 1980:25examined.

Diagnosis: Meteorus cinctellus has a blunt tubercle in front of the fore ocellus, the pterostigma is dark brown with white edges, the subapical antennal articles are as longer as broad and the ovipositor is 2.0-2.5 times petiolar tergum. Other similar species, such as M. tenellus, M. artocercus and M. colon, also have the blunt tubercle but have a pale pterostigma without white edges.

Studied material: $\sim 100$ specimens.

Description: Size about 4mm. Antennae with 24-29 articles; Subapical antennal articles as longer as broad. Frons depressed with a blunt tubercle in front of the fore ocellus. Ocelli small, OOL=2.5. Eyes large, protuberant and strongly convergent. Malar space shorter than the basal breadth of the mandible. Face about as high as broad with a medial tubercle just below the antennal sockets. Clypeus narrow, strongly protuberant. Tentorial pits deep. Mandible small and strongly twisted. Precoxal sulcus deep, foveolate and generally broadened anteriorly. Propodeum rather flat, regularly reticulate-rugose, sometimes with a strong medial transverse carina. Petiolar tergum long, dorsal pits absent. Ventral borders generally touching but sometimes open. Ovipositor 2.0-2.5 times length of petiolar tergum. Legs long and slender; hind coxae rugose. Tarsal claws small and with a distinct basal lobe. Colour black/yellow; legs and generally $3^{\text {rd }}$ abdominal tergite testaceous. ${ }^{\lambda}$ antenna 28-30 articles; ocelli slightly larger; eyes less strongly convergent; colour often darker.

Distribution: Palearctic. Country records: Albania; Austria; Belgium; Bulgaria; China; Czechoslovakia; Denmark; Finland; France; Germany; Greece; Hungary; Ireland; Italy; Japan; Korea; Latvia; Madeira Islands; Netherlands; New Zealand; Norway; Poland; Portugal; Romania; Slovenia; $\underline{\text { Sweden; Switzerland; Ukraine; United }}$ Kingdom; Yugoslavia.

Biology: Meteorus cinctellus parasitizes on Tortricidae (Lepidoptera) (Yu et al. 2005). We found 99 specimens within the SMTP; they were caught in a variety of biotopes, including deciduous and coniferous forests. Flying time is from June to September.

Remarks: The name Ichneumon necator (Fabricius, 1777) is not the type of M. cinctellus (Yu et al. 2005, Broad et al. 2009). The name necator (Fabricius, 1777) refers to the Microgastrinae species Apanteles necator and is not a Euphorinae. In the description of Ichneumon necator (Fabricius, 1777:246) there is a reference to one of Rösel's illustrations (Table IV Fig. 4 in Rösel). The depicted braconid is clearly a Microgastrinae. 


\section{Meteorus cis (Bouché)}

Fig. 91

Bracon cis Bouché, 1834:149. Type material unknown.

Perilitus profligator Haliday, 1835:33. Syntypes $q$ Ireland (lost). Synonymized by Fahringer 1928:505.

Diagnosis: Meteorus cis is similar to M. breviantennatus but the clypeus of the latter is almost as wide as the face while the clypeus of M. cis is narrow. Also the malar space (shorter than mandibular base on M. breviantennatus, as wide as mandibular base on $M$. cis) and the face (slightly more transverse face in M. brevianennatus, round face in M. cis) should separate these species.

Studied material: $\sim 20$ specimens.

Description: Size about 2.5-3.5mm. Antennae 20-22 articles. Head rounded behind eyes. Ocelli small, $\mathrm{OOL}=3$. Eyes small, not strongly protuberant, slightly convergent. Malar space slightly shorter than basal breadth of mandible. Face 1.5-2.0 times as broad as high, smooth, punctate, not strongly protuberant. Clypeus narrower than face, almost flat, its apical border weakly notched medially and usually with a weak vertical impression behind the notch. Tentorial pits distinct. Mandibles short, stout and moderately twisted. Precoxal sulcus foveolate, sometimes broadening anteriorly. Mesopleuron dorsally reticulate-rugose, otherwise smoothly punctate. Propodeum with carinae with very weak rugose sculpture in between. Petiolar tergum with large distinct dorsal pits, dorsal surface generally reticulate-rugose with a few longitudinal striae laterally. Ovipositor about twice the length of petiolar tergum, slender. Legs slender; hind coxa never strongly rugose but often with a trace of obsolescent rugosity at the base and on the outer surface. Tarsal claws not lobed but somewhat swollen basally. Colour testaceous to brown; clypeus, mandibles and usually $3^{\text {rd }}$ abdominal tergite reddish testaceous; coxa and legs yellow. Pterostigma dark with light border around, more so anteriorly. Fore wing $\mathrm{m}-\mathrm{cu}$ antefurcal, vein $2-\mathrm{SR}+\mathrm{M}$ almost as long as vein $\mathrm{r}$, at most 0.7 times).

Distribution: Palearctic. Country records: Austria; China; France; Germany; Ireland; Japan; Korea; Netherlands; Sweden; United Kingdom.

Biology: Meteorus cis is recorded as a parasitoid of Cis boleti Scopoli, 1763 (Bouché 1834). We found 12 specimens within the SMTP; they were caught in both deciduous and coniferous forests. Flying time is from July to October.

\section{Meteorus colon (Haliday)}

Fig. 10b, 20, 30, 92

Perilitus colon Haliday, 1835:30. Syntypes $\odot$, Ireland (lost).

Perilitus fragilis Wesmael, 1835:52 Lectotype ${ }^{\lambda}$, Belgium: Brussels (IRSNB, Brussels). Synonymized by Huddleston, 1980:26.

Perilitus fasciatus Ratzeburg, 1844:77. Syntypes 9 , Germany (lost). Synonymized with fragilis by Ruthe, 1862:55.

Meteorus luridus Ruthe, 1862:57 Lectotype + , Germany: Ruthe coll. (BMNH, London) - examined

Diagnosis: Meteorus colon has a blunt tubercle on frons in front of the fore ocellus. This character is shared with M. cinctellus, $M$. tenellus, $M$. artocercus, $M$. stenomastax and M. melanostictus. M. colon is distinguished from these other species by having a distinctly postfurcal second submarginal cell and a pale pterostigma.

Studied material: 200 specimens.

Description: Size about $4 \mathrm{~mm}$. Antennae long, 30-34 articles, all flagellar articles long. Head contracted behind eyes; frons with a blunt tubercle in front of the fore ocellus. Ocelli rather large but not conspicuously so, $\mathrm{OOL}=2$. Eyes convergent but not strongly so. Malar space short, slightly less than basal breadth of mandible. Face not strongly protuberant. Clypeus strongly protuberant; narrower than face. Mandibles small, strongly twisted. Precoxal sulcus foveolate. Propodeum finely rugose, carinae often weak and indistinct. Petiolar tergum long, slender, without dorsal pits, the ventral borders generally slightly separated. Ovipositor 1.5 times length of petiolar tergum. Legs very long and slender; tarsal claws small with a distinct basal lobe. Forewing m-cu distinctly postfurcal, the second submarginal cell rather elongate. Colour black to yellow; $\widehat{\jmath}$ antenna $31-35$ articles. 
Distribution: Western and Eastern Palearctic. Country records: Armenia; Austria; Azerbaijan; Belgium; Bulgaria; China; Croatia; Czechoslovakia; Denmark; Finland; France; Georgia; Germany; Hungary; Ireland; Italy; Japan; Lithuania; Moldova; Netherlands; Norway; Poland; Russia; Slovakia; Sweden; Switzerland; Turkey; United Kingdom; Yugoslavia.

Biology: Host records of Meteorus colon include ten different families of Lepidoptera, with most records involving species of Noctuidae (Huddleston 1980, Yu et al. 2005). We found 124 specimens within the SMTP; they were caught in both deciduous and coniferous forests but predominantly in spruce forests. Flying time is from July to October.

\section{Meteorus consimilis (Nees von Esenbeck)}

Fig. 61, 62, 93

Perilitus consimilis Nees von Esenbeck, 1834:42. Syntypes $\hat{\alpha}$, Germany (destroyed).

Perilitus brevipes Wesmael, 1835:33 Lectotype $\widehat{\partial}$, Belgium: Brussels, coll. Wesmael (IRSNB, Brussels), Synonymized with albicornis Ruthe by Marshall, 1887:110.

Meteorus albicornis Ruthe, 1862:34. lectotype + , Germany: Ruthe coll. (BMNH, London). Synonymized with consimilis by Thomson, 1895:2160-examined

Diagnosis: The only species that could be confused with this species is M. abdominator. A good separating character is the length of the malar space (almost two times basal breadth of mandible in M. consimilis, at most as long as basal breadth of mandible in M. abdominator. The length and shape of the ovipositor also differ between these species. The ovipositor of M. consimilis is thick, distinctly down-curved and short (equal in length to petiolar tergum), while M. abdominator has a thin and long (2.0-2.5 times length of petiolar tergum), and only slightly down-curved ovipositor.

Studied material: $\sim 60$ specimens.

Description: Size about 3-4mm. Antennae 32-33 articles; basally whitish. Head strongly contracted behind eyes. Ocelli large, $\mathrm{OOL}=1.5$. Eyes small, not convergent. Malar space long, almost twice basal breadth of mandible. Clypeus strongly protuberant. Tentorial pits deep. Mandibles slender; strongly twisted. Precoxal sulcus deep, broad, reticulate-rugose. Propodeum strongly reticulate-rugose. Petiolar tergum short, wide, with distinct dorsal pits. Ovipositor short, at most equal in length to petiolar tergum; thick down-curved. Wings infumate and short. Colour dark. $\delta$ antennae $32-36$ articles with no pale band.

Distribution: Western Palearctic. Country records: Belgium; Croatia; Denmark; Finland; France; Germany; Hungary; Ireland; Latvia; Netherlands; Poland; Romania; Slovenia; Sweden; Switzerland; Turkey; Turkmenistan; Ukraine; United Kingdom; Yugoslavia.

Biology: Meteorus consimilis is recorded as a parasitoid of Scolytus multistriatus (Yu et al. 2005). We found 58 specimens within the SMTP; they were caught in both deciduous and coniferous forests. Flying time is from middle of July to end of October.

\section{Meteorus corax Marshall}

Fig. 51, 77, 94

Meteorus corax Marshall, 1898:220. Lectotype $\odot$ Switzerland: Val Somvix/25.vii.1891/Marshall det. (NMB, Bern) Meteorus monachami Fischer, 1957c:17. Holotype $\odot$ Austria: Lunz (Haberfelner), ex Monochamus saltuarius Gebl. (ZSBS, Munich) Synonymized by Fischer, 1966:395.

Diagnosis: This is one of the larger species of Meteorus, at least in the Palaearctic Region, averaging about $9 \mathrm{~mm}$ in body length. It is most easily confused with M. nixoni. However, M. corax has twisted mandibles (straight in $M$. nixoni), the temples are wider than in $M$. nixoni, and the $3^{\text {rd }}$ abdominal tergum is densely covered with striae (only a few striae present in M. nixoni).

Studied material: $\sim 10$ specimens.

Description: Size about 6-10mm. Antennae 39-41 articles. Head rather square. Ocelli small, OOL=3. Eyes small, converging only very slightly. Face about twice as long as broad. Clypeus distinctly narrower than face; pro- 
tuberant. Tentorial pits wide and deep. Mandibles very stout and slightly twisted, with striate-punctate sculpture and long setae. Precoxal sulcus deep, foveolate with, anteriorly, a reticulate-foveolate patch beneath. Propodeum strongly reticulate-rugose with basal and medial transverse carinae. Petiolar tergum with longitudinal sculpture laterally, reticulate-rugose centrally; spiracles distinctly before the middle; large dorsal pits present, borders of petiolar tergum not meeting ventrally. The ovipositor about 4 times length of petiolar tergum. Outer surface of hind coxa and femur densely reticulate - punctate; hind tibia strongly swollen though not as wide as femur; tarsal claws large, strongly curved, not lobed but strongly swollen at base. Colour black or dark brown.

Distribution: Palearctic. Country records: Austria; Finland; Germany; Italy; Japan; Korea; Russia; Sweden; Switzerland.

Biology: Most host records of Meteorus corax involve the coleopteran family Cerambycidae (Huddleston 1980).

\section{Meteorus densipilosus Stigenberg sp. nov.}

Fig. 79B, 95

Diagnosis: The clypeus of Meteorus densipilosus is unique within Meteorus in being flat and having a sinuate ventral margin with a median notch similar to that of M. cis. The setose face, straight ovipositor, and distinct carinae on the propodeum are also good characters that distinguish it from related species. In Huddleston's (1980) key, $M$. densipilosus runs to $M$. brevicauda, but there are distinct differences. While $M$. brevicauda belongs to the smallest of the Meteorus species (less than $3.5 \mathrm{~mm}$ ), M. densipilosus is larger at about $5 \mathrm{~mm}$. M. densipilosus has a distinct malar space ( 0.5 times basal width of mandible) while $M$. brevicauda hardly has any space at all between the eye and the mandible. The ovipositor is also longer in M. densipilosus (2.0 times petiolar tergum) than in M. brevicauda (1.5 times petiolar tergum). In our key, this species groups with $M$. eklundi, but it is clearly separated from the latter based on the number of antennal articles (28 in M. densipilosus and only 22-25 in M. eklundi).

Studied material: 1 q . Holotype: $1 \propto$ SE. Sm. Nybro kommun. Bäckebo. 24.viii-12.ix.2005. (leg. SMTP), NHRS-HYME 4936, DNA 7.

Description: Body $5.2 \mathrm{~mm}$, fore wing $3.47 \mathrm{~mm}$.

Head: Length of eye 0.9 times length of temple in dorsal aspect; eyes small, protuberant, not converging; width of face 1.7 times its height; $\mathrm{OOL}=2.5$, ocelli only slightly protruding; vertex smooth, convex but with a ridge running towards face; face striate medially, the striae running to clypeus forming ridges; clypeus as wide as face, not distinctly divided from face and flat with ventral border sinuate seen from dorsal view; length of malar space 0.5 the times basal width of mandible; mandible stout, not twisted; hypostomal carina wide; antennal articles 28; 3rd antennal article 4.75 times longer than wide; 4th antennal article 3.2 times longer than wide; all antennal articles longer than wide.

Mesosoma: Precoxal sulcus wide.

Wings: Fore wing: $\mathrm{r} / 3-\mathrm{SR}=0.6 ; \mathrm{C}+\mathrm{SC}+\mathrm{R} / \mathrm{SR} 1=1.48 ; \mathrm{m}-\mathrm{cu}$ antefurcal; pterostigma dark brown with light dorsal border. Hind wing: $1-\mathrm{M} / \mathrm{cu}-\mathrm{a}=1.6$.

Legs: Hind coxa rugose; hind femur 5 times longer than wide; tarsal claws slender without lobe.

Metasoma: Petiolar tergum 1.85 times longer than wide apically, 4.5 times fore wing; dorsope present; ventral folds of petiolar tergum not joined; ovipositor slender and straight, length of ovipositor sheaths 2.0 times petiolar tergum and 0.45 times fore wing; propodeum convex, reticulate rugose and with 2 distinct transversal and one medial longitudinal carina; propodeum not strongly excavate medially.

Colour: Black except: yellow patch between eye and posterior ocelli; gena dark brown; clypeus and palpi yellow; antennae brown; petiolar tergum black and metasoma dark brown; legs yellow.

Distribution: Sweden.

Biology: The specimen was caught in a Malaise Trap in late August - early September in southern Sweden. The locality, Grytsjöns naturreservat, mostly consists of an old aspen forest in boulder terrain.

Etymology: The name densipilosus refers to the dense setosity on the head, being composed of the words densi (Latin, meaning thick), and pilosus (Greek, meaning hairy). 


\section{Meteorus eadyi Huddleston}

Fig. 41, 96

Meteorus eadyi Huddleston, 1980:29. Holotype $q$ Great Britain: Northhamptonshire, Spratton, x.1975 (Gauld) (BMNH, London)-examined.

Paraytpes. Bulgaria: 1 , Rhodopi, Markovo, 13.viii.1977 (Zaykov) (PZ, Plavodiv). Great Britain: 17 os, 1 ऽ, England, Northhamptonshire, Spratton, v.-x.1975 (Gauld) 1 female, E., oxfordshire, Waterferry Common, 5.viii.1969 (Brock) (BMNH); 1 , Greater London, Kew, 22.vii.1979 (Eastop) (BMNH). Germany: 1 ․, Bonn, Waldhauhohenweg, 18.xi.1960 (Schmidt) (CNC, Ottawa). Netherlands: 6 females, Wijster (opp, biol. station); 3, 13-26.viii.1973; 1, 7-14.vii.1974; 10-17.ix.1976; 1. 16-23.vi.1978 (van Achterberg) (RNH, Leiden); 7 s, Waarder (ZH), Oosteinde; 1, 1-15.ix.1973; 1, 17-22.vi.1974; 1, 1-5.viii.1974;2, 1-6.ix.1974; 1, 15-22.vi.1975; 1, 21-31.x.1975 (van Achterberg) (RNH, Leiden); 1 ○, Ede, $15 . i x .1970$ (Zwakhals) (RNH, Leiden). Yugoslavia: 1 \%, Plitvice, 4-10.vii.1955 (Coie) (BMNH).

Diagnosis: Meteorus eadyi is close to $M$. filator but can be distinguished by its smaller, less convergent eyes, and by the shape of the clypeus being narrow and protruding, not wide and flat as in M. filator. The antennae (26-27 articles) are long and slender in M. eadyi, while M. filator has stouter antennae with about 21-25 articles. The ovipositor is 1.5 times petiolar tergum in M. eady and 2.0 times petiolar tergum in M. filator.

Studied material: $\sim 20$ specimens.

Description: Size 4-5mm. Antennae 26-27 articles, all articles longer than broad. Head rounded behind eyes. Ocelli small, OOL=2.5-3.0. Eyes small, protuberant and not strongly convergent. Face about 1.5 times as broad as high. Clypeus not strongly protuberant. Mandibles not twisted, the upper tooth rather long. Precoxal sulcus foveolate. Propodeum carinated with three longitudinal and two transverse carinae. Petiolar tergum long, slender, about twice as long as apically broad; no dorsal pits; ventral borders of petiolar tergum conjoined. Ovipositor short, about 1.5 times as long as petiolar tergum, down-curved. Legs slender, hind coxae partly rugose; tarsal claws without a basal lobe. Colour black with yellow parts, basal antennal articles usually slightly lighter than the rest. Male same as female except that the eyes are smaller and less convergent.

Distribution: Western Palearctic and Mongolia. Country records: Armenia; Bulgaria; Czechoslovakia; France; Germany; Hungary; Korea; Lithuania; Mongolia; Netherlands; Romania; Russia; Sweden; Switzerland; United Kingdom; Yugoslavia.

Biology: Meteorus eadyi is attracted to light (Yu et al. 2005). It flies during June to September and the host is unknown.

Remarks: M. eadyi is here reported as new for Sweden. In the material from MZLU we found 1 from Skåne.

\section{Meteorus eklundi Stigenberg sp. nov.}

Fig. 68, 79C, 97

Diagnosis: Meteorus eklundi can be distinguished from related species by the stout antenna with articles that are quadrate in shape. M. eklundi mostly resembles M. nadezdhae (East Russia) but the latter species has a rugose face, with the rugosity extending down onto the clypeus, and a strongly rugose petiolar tergum, whereas $M$. eklundi is only equipped with light sculpture in these body regions. M. eklundi also resembles M. obfuscatus but differs from this species by larger and more convergent eyes, narrower face (face wider than high in the latter) and shorter malar space ( 0.4 times basal width of mandible in the former, 0.5 times in the latter).

\section{Studied material: 12 .}

Holotype: 1 q SE. Sm. Älmhults kommun. Stenbrohult. 26.vi-15.vii.2003. (leg. SMTP), NHRS-HYME 4937, DNA 8. Paratype: 1 SE. Öl. Mörbylånga kommun. Kalkstad. 25.vi-13.vii.2003. NHRS-HYME 4939, DNA 28. 1 ○ SE. Öl. Mörbylånga kommun. Kalkstad. 2.v-18.vi.2006 (leg. SMTP), NHRS-HYME 4938.4 q specimens at ZIN collected in Belarus, earlier misidentified as Paratypes of $M$. nadezdae, these are included as Paratypes of $M$. eklundi. 1 \& Netherlands: N.B. Udenhoult, "De Brand" 26.v.2.VII.1990 UTM FT 476225, Mal.trap Ins. W.G. KNNV-Tilburg. 1q SE Bulgaria (Mal tr 1) Brodilovo, nr Achtopol c 20 m, 7.v-8.vi.1998, C.v.Achterberg, R de Vries, P.V. Atanassova, RMNH'98. 1q Västerbotten, Romelsön RN7208/1759 Mal.trap 21.6-29.8.03. R. Petterson. 1 q Chippenham Fen, Cambs. TL 650693 Malaise trap: carr at reedbed edge.A. 9-20.07.84. J. Field. RMSNH 1986.021. 1 q Chippenham Fen, Cambs. TL 650693 Malaise trap: carr at reedbed edge.A. 25.06-09.07.85. J. Field. RMSNH 1986.021. 
Description: Body $4.2 \mathrm{~mm}$, fore wing $3.5 \mathrm{~mm}$.

Head: Length of eye 1.5 times length of temple in dorsal aspect; eyes large, convergent, not strongly protuberant; width of face 1.5 times its height; $\mathrm{OOL}=3$, ocelli only slightly protruding; vertex smooth; face punctulate; clypeus as wide as face, distinctly divided from face, punctuate, flat with slight notch; length of malar space 0.4 times basal width of mandible; mandible stout, slightly twisted; hypostomal carina wide; number of antennal articles 22 (21-25); $3^{\text {rd }}$ antennal article 2 times longer than wide; 4th antennal article 1.4 times longer than wide; penultimate articles as wide as broad or slightly wider.

Mesosoma: Precoxal sulcus wide.

Wings: Fore wing: $\mathrm{r} / 3-\mathrm{SR}=0.4 ; \mathrm{C}+\mathrm{SC}+\mathrm{R} / \mathrm{SR} 1=1.6 ; \mathrm{m}-\mathrm{cu}$ antefurcal; pterostigma dark brown with light border. Hind wing: $1-\mathrm{M} / \mathrm{cu}-\mathrm{a}=1.2$;

Legs: Hind coxa rugose; hind femur 5 times longer than wide; tarsal claws slender without lobe.

Metasoma: Petiolar tergum 1.8 times longer than wide apically, 4.2 times fore wing; dorsope present; ventral folds of petiolar tergum not joined; ovipositor weakly curved downward, length of ovipositor sheaths 1.6 times petiolar tergum, and 0.45 times fore wing; propodeum strongly convex, reticulate rugose and with no distinct carina; strongly excavate medially.

Colour: Black except: clypeus and palpi yellow; antennae brown; petiolar tergum and metasoma dark brown; legs yellow to brown; hind coxa darker brown at base.

Distribution: Western Palearctic. Country records: Belarus; Bulgaria; Netherlands, Sweden; United Kingdom.

Biology: Meteorus eklundi is caught in malaise traps during the summer months June, July and August. The three specimens that were caught within the SMTP were found in localities with deciduous forests. Host unknown.

Etymology: Named after my best friend Mr Niclas Eklund.

\section{Meteorus filator (Haliday)}

Fig. 18, 24, 40, 98

Perilitus filator Haliday, 1835:32. Holotype + , Ireland: 'British Haliday 20.2.82' (NMI, Dublin)

Perilitus laticeps Wesmael, 1835:47. Lectotype ${ }_{+}$, Belgium: Brussels, coll. Wesmael (IRSNB, Brussels), Considered valid species by Fischer, 1970a. Synonymized by Huddleston, 1980:30.

Meteorus hodisensis Fischer, 1970b:285. Holotype ${ }_{+}$, Austria: 'Bgld., Markt. Hodis, Rechnitz, 7.viii.1961' (Fischer) (NHM, Vienna). Synonymized by Huddleston, 1980:30-examined.

Diagnosis: Males of Meteorus filator look very similar to those of M. eadyi but the shape of the clypeus is a good diagnostic character (wide in M. filator, narrow and protruding in M. eadyi).

Studied material: $\sim 60$ specimens.

Description: Size about $4.5 \mathrm{~mm}$. Antennal articles 21-25. Head broad, temples rounded. Ocelli small, $\mathrm{OOL}=2.5-3.0$. Eyes large, strongly convergent. Face about as wide as high, not strongly protuberant. Clypeus wide, not strongly protuberant. Malar space short, less than half the basal breadth of mandible. Mandible large, not twisted. Precoxal sulcus wide. Propodeum rather depressed with three longitudinal and two transverse carinae. Petiolar tergum long, almost equal in length to rest of abdomen, slender, ventral borders joined from the base of the segment to its midpoint. Ovipositor long, 2.5 times length of petiolar tergum. Legs long, slender; hind coxa partly reticulate-rugose; tarsal claws simple, long, slightly swollen at base. Colour mostly black. Male same as female except eyes are smaller and less convergent so that the face is about twice as broad as high and the malar space longer; antennae longer, 27-30 articles, all articles of flagellum at least twice as long as broad, darker in colour, rarely distinctly yellow in basal half of flagellum.

Distribution: Western Palearctic. Country records: Austria; Azerbaijan; Belgium; Bulgaria; Czechoslovakia; Denmark; Finland; France; Georgia; Germany; Hungary; Ireland; Italy; Lithuania; Mongolia; Netherlands; Norway; Poland; Russia; Slovakia; Sweden; Switzerland; United Kingdom.

Biology: Haliday (1835) states "locis fungiferis autumno" for this species, meaning that it occurs amongst fungi in autumn. Also, Capron mentions to Marshall (1887) that he has taken several females of this species by shaking species of Trametes versicolor (Fungi, Polyporaceae) and the males were abundantly collected in the autumn by sweeping. We found 41 specimens within the SMTP, most of them were from a Malaise trap placed on a sandy railway. They were caught during the summer months July to August. 


\section{Meteorus heliophilus Fischer, 1970}

Fig. 115

Meteorus heliophilus Fischer, 1970b:284. Holotype + , Austria: Burgenland, Reichnitz, 2.viii.1958 (Fischer) (NM, Vienna).

Diagnosis: Similar to M. rubens but M. heliophilus has a larger marginal cell and longer antennae with 30-32 antennal articles (24-28 in M. rubens).

Studied material: $\sim 10$ specimens.

Description: Size about $4 \mathrm{~mm}$. Antennae long with 30-32 articles. All articles much longer than broad. Head strongly contracted behind eyes. Eye in dorsal view 2.5-3.0 times as long as temple. Ocelli large OOL=1.0-1.5. Eyes large, not strongly convergent but protuberant. Malar space short, less than half the basal breadth of mandible. Face about as high as broad, not strongly protuberant, slightly raised medially where it is transversely rugose, laterally punctuate. Clypeus protuberant but not strongly, smooth with scattered coarse punctures. Tentorial pits small, indistinct. Mandibles strongly twisted, the upper tooth not long. Pronotum laterally smooth with a little fine rugose sculpture medially. Mesonotum punctate, sometimes the central lobe reticulate-punctate, at least in part; notaulices weakly impressed. Precoxal sulcus weakly rugose, sometimes obsolescent posteriorly. Propodeum shining with weak carinae and weak, largely obsolescent rugae dorsally. Petiolar tergum long, slender, ventral borders meeting beneath at the mid-part of the segment, not joined at the base of the segment; dorsal surface weakly longitudinally striate; no glymmae or dorsal pits. Ovipositor about twice the length of petiolar tergum, thick, strongly expanded at the base and the apical sixth much narrowed. Legs long, slender; hind coxa mainly smooth but sometimes with a trace of rugosity dorsally. Colour testaceous, the legs generally a paler shade of yellow. Male same as female except antennae 31-33 articles.

Distribution: Palearctic. Country records: Austria; Belgium; Bulgaria; China; Czechoslovakia; Denmark; Finland; France; Germany; Hungary; Italy; Japan; Latvia; Moldova; Mongolia; Poland; Slovakia; Sweden; Switzerland; United Kingdom.

Biology: M. heliophilus is a gregarious parasitoid (Tobias 1976, Huddleston 1980). Hosts: Polia nebulosa (Hufnagel), Litophane ornitopus (Hufnagel), Noctua fimbriata (Schreber), Xestia triangulum (Hufnagel), Orthosia stabilis (Denis \& Schiffermüller) (Lepidoptera, Noctuidae) (Huddleston 1980).

\section{Meteorus hirsutipes Huddleston}

Fig. 21, 67, 99

Meteorus hirsutipes Huddleston, 1980:34. Holotype $q$ Finland: Valamo, Ladoga, NE. of monastery Hainwald 24.viii.1935 (Kerrich) (BMNH, London) examined.

Paratypes. Germany: 1 B Bavaria, Schliersee, 28.vii.1958 (Townes) (CNC, Ottawa). Ireland: 1 , Co. Wicklow, Deputy’s Pass, 7.viii.1932 (Stelfox) (USNM, Washington).

Diagnosis: Meteorus hirsutipes is an easily diagnosed species. It is a rather large species with an unusually wide face. The colour is black except for the prothorax and legs, which are testaceous. Above all, however, the species is characterized by the very long, sickle-shaped claws.

Studied material: $\sim 20$ specimens.

Description: Size about 4.5-6mm. Antennal articles 28-30, thin. Head strongly narrowed behind eyes, occiput concave. Ocelli small, OOL=3. Eyes protuberant, slightly convergent. Face about twice as broad as high, not strongly protuberant. Clypeus not strongly protuberant, as broad as face. Mandible very large, stout and not twisted. Precoxal sulcus deep, foveolate. Propodeum with strong longitudinal and transverse carinae. Petiolar tergum with longitudinal striae, joining to form a transverse rugose area; medial part of petiolar tergum anteriorly reticulate- rugose. Ovipositor 2.5 times length of petiolar tergum, straight. Hind coxa punctate or reticulate-punctate; tarsi with long setae, the longest of which at the apices of the tarsal segment are at least twice the maximum breadth of the segment. Tarsal claws simple, very long, sickle-shaped. Colour black, prothorax and legs testaceous.

Distribution: Western and Eastern Pelearctic. Country records: China; Croatia; Finland; Germany; Ireland; Japan; Norway; Switzerland; Sweden; United Kingdom. 
Biology: We found 5 specimens within the SMTP and they were all caught in August in a mixed forest.

\section{Meteorus ictericus (Nees von Esenbeck)}

Fig. 70, 71, 74, 100

Bracon ictericus Nees von Esenbeck, 1811.22 Syntypes $\odot$, Germany (destroyed).

Ichneumon minutor Thunberg, 1822.266. Holotype + (UDE, Uppsala) Synonymized by Roman 1912:267.

Zele ephippium Curtis, 1832: folio 415. Syntypes, Great Britain:'Coomb Wood' (NMV, Melbourne). Synonymized by Curtis 1837.

Perilitus xanthomelas Wesmael, 1835:29. Lectotype $\hat{\jmath}$, Belgium: Brussels (IRSNB, Brussels). Synonymized by Fischer, 1970b:263.

Meteorus confinis Ruthe, 1862:18. Lectotype +, Germany: Ruthe coll. (BMNH, London). Synonymized by Huddleston, 1980:34.

Meteorus fallax Ruthe, 1862:18. Lectotype ${ }_{+}$, Germany: Ruthe coll. (BMNH, London). Synonymized by Huddleston, $1980: 34$.

Meteorus pleuralis Ruthe, 1862:19. Lectotype ${ }_{+}$, Germany: Ruthe coll. (BMNH, London). Synonymized by Huddleston, 1980:34.

Meteorus liquis Ruthe, 1862:20. Lectotype ${ }_{+}$, Germany: Ruthe coll. (BMNH, London). Synonymized by Huddleston, $1980: 34$. Meteorus consors Ruthe, 1862:44. Lectotype $\circ$, Germany: Ruthe coll. (BMNH, London) examined. Synonymized by Fischer, 1970b:263.

Meteorus crassicrus Thomson, 1895:2154. Lectotype 9 , Sweden: Skåne, Arrie (MZLU, Lund) - examined

Meteorus lophyriphagus Fahringer in Schönwiese, 1934:495. Holotype $\odot$, Austria (IFF, Vienna)

Meteorus adoxophyesi Minamikawa, 1954:41. Holotype +, Japan: Kanaya Shizuoka-ken, 10.iv.1948 (Sonan) (NIAS, Yatabe) Synonymized by Huddleston, 1980:34.

Diagnosis: Meteorus ictericus is most similar to M.ruficeps but the latter species has converging eyes, whereas the eyes of M. ictericus are only slightly converging. M. ictericus can sometimes be hard to separate from M.pendulus but $M$. ictericus has whitish hairs between the eyes and the clypeus (absent in $M$. pendulus), rugose glymmae (smoother in M. pendulus) and longer ovipositor (2.5-3.0 times length of petiolar tergum in M. ictericus, 1.5-2.0 times petiolar tergum in M. pendulus).

Studied material: $\sim 200$ specimens.

Description: Size about 4.5-5.5mm. Antennal articles 26-33, long. Head strongly contracted behind the eyes. Ocelli large, $\mathrm{OOL}=1.0-1.5$, protuberant, the ocellar area raised and there is a pit in front of anterior ocelli, the size of the pit varies. Eyes large, protuberant, inner margins converging very slightly. Malar space short, at most equal to half basal breadth of mandible. Face often only slightly raised medially. Clypeus narrower than face, protuberant. Tentorial pits small. Mandibles moderately twisted, short and rather stout. Precoxal sulcus foveolate, narrow. The propodeum is excavated with a strong medial transverse carina marking the excavation, the weak medial longitudinal carina is surrounded by an area on both sides with smooth or very faint structure. Petiolar tergum long, slender with distinct dorsal pits and rugose glymmae. Ovipositor long, 2.5-3.0 times length of petiolar tergum, stout. Legs long, slender; hind coxa smooth, tarsal claws strongly bent and with a large basal lobe. Colour varying. Males look the same as females, the only one we studied had antennae with 28 articles. Males are very rare and only one was found in the SMTP.

Distribution: Palearctic. Country records: Armenia; Australia; Austria; Azerbaijan; Belarus; Belgium; Bulgaria; China; Croatia; Czechoslovakia; Denmark; Finland; France; Germany; Hungary; Ireland; Israel; Italy; Japan; Kazakhstan; Korea; Latvia; Lithuania; Moldova; Netherlands; Norway; Poland; Romania; Russia; Slovakia; Slov-

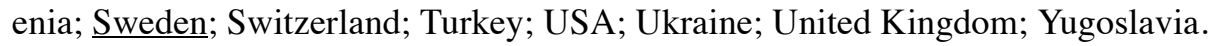

Biology: Meteorus ictericus is readily caught in Malaise Traps but is also attracted to light. Host records include 14 different lepidopteran families, but the majority of records involve the Tortricidae (Huddleston 1980, Yu et al. 2005). We found 89 specimens (only one male) within the SMTP and they were caught in forests, both deciduous, coniferous and mixed forests, though with a slight preference towards spruce forests. The period of flight seems to be from June to October. 


\section{Meteorus ipidivorus Tobias}

Fig. 54, 101

Meteorus ipidivorus Tobias 1986:340, 351 Holotype ${ }_{+}$, Russia:

Diagnosis: Meteorus ipidivorus belongs to the group of smaller Meteorus species with body length less than 3.5 $\mathrm{mm}$. It is easily distinguished by being the only small Meteorus with the fore wing RS bent upward.

Studied material: 1 specimen

Description: Size about 3mm. Antennal articles 28-29. Head rounded behind eyes, ocelli very minute $\mathrm{OOL}=4$. Eyes smaller, converging slightly. Malar space 0.5 times mandible base. Mandibles stout and not twisted. Face 1.5 times broader than high, not protruding. Clypeus flat and as wide as face. Precoxal sulcus broad, punctuated otherwise smooth. Propodeum carinated but smooth inbetween. Fore wing 4 times the length of petiolar tergum. Petiolar tergum with dorsal pits, rugose dorsally. Ovipositor short, slightly longer than petiolar tergum and curved. Hind coxae rugose, dark brown. Fore wing radial vein not straight, stigma brown.

Distribution: Russia

Biology: Meteorus ipidivorus is recorded as a parasitoid of Ips acuminatus and Blastophagus minor (Curculionidae) (Tobias 1986). The specimen studied here is the holotype from ZIN and this specimen was found in the gallery of Ips acuminatus in Novosibirsk in September 1965.

\section{Meteorus jaculator (Haliday)}

Fig. 102

Perilitus jaculator Haliday, 1835:34. Syntypes $\circ$, Ireland (lost).

Meteorus obscurellus Ruthe, 1862:29. Holotype + , Germany: Ruthe coll. (BMNH, London) examined. Synonymized by Marshall, 1887:108.

Meteorus tenuicornis Thomson, 1895:2164. Lectotype +, Sweden: Skåne, Pålsjö (ZI, Lund) - examined

Diagnosis: Meteorus jaculator is similar to $M$. cis and $M$. vexator. However, M. vexator is easily distinguished from the other two based on the strongly converging eyes. The eyes are only slightly converging in the other two species, which are most easily distinguished by the length of the ovipositor. $M$. jaculator has an ovipositor length of 3-4 times the length of the petiolar tergum, whereas $M$. cis has a much shorter ovipositor, only 2 times the length of the petiolar tergum.

Studied material: $\sim 20$ specimens.

Description: Size about 3mm. Antennae short, 18-22 articles, slender. Head swollen behind eyes; temples longer than eyes. Ocelli small, OOL=3-4. Eyes small, slightly convergent. Malar space at least slightly shorter than basal breadth of mandible. Face about twice as broad as high, protuberant. Clypeus wide as face and smooth, with scattered large punctures, not strongly protuberant. Tentorial pits small. Mandibles stout, slightly twisted. Precoxal sulcus narrow. Propodeum with weak but distinct carinae. The medial longitudinal area of the petiolar tergum is strongly raised ending apically in reticulate rugosity. Ovipositor 3-4 times length of petiolar tergum. Hind coxa often strongly rugose. Tarsal claws short with no basal lobe. Colour dark brown to black; legs testaceous but sometimes the coxae darker; clypeus and mandibles often yellow. Male same as female except that the antennae are much longer, up to 29 articles, all flagellar articles conspicuously longer than broad. Fore wing vein $r$ is as long as vein 3-SR and $\mathrm{m}$-cu antefurcal.

Distribution: Western Palearctic. Country records: Austria; Bulgaria; Croatia; Czechoslovakia; Denmark; Finland; France; Germany; Hungary; Ireland; Netherlands; Norway; Poland; Russia; Slovakia; Sweden; Switzerland; Turkey; United Kingdom; Yugoslavia.

Biology: M. jaculator is recorded as being a parasitoid on three lepidopteran families (Gelechiidae, Psychidae and Tineidae) (Yu et al. 2005). We found 6 specimens within the SMTP. They were caught from July to October in northern Sweden, predominantly in deciduous forests. 


\section{Meteorus kyushuensis Maeto}

Fig. 103

Meteorus kyushuensis Maeto, 1988:328. Holotype , (Type No. 2567, Kyushu Univ.) Japan: Gokanosho, Kumamoto Pref., Kyushu 14.v.1980, K. Ohara [L.T.] — examined.

Diagnosis: In Huddleston's key this species runs to M. hirsutipes, and it also groups with this species in our key. The main characters separating these species are the claws (long and slender in M. hirsutipes, of ordinary length in M. kyushuensis) and the clypeus (very flat and wide in M. hirsutipes, flat but not at all wide in M. kyushuensis).

Studied material: 5 specimens.

Description: Size about 3.0-4.5. Antennal articles 23-24. Head broad, rounded behind eyes. Length of eye 1.4-1.7 times temple. Ocelli small, $\mathrm{OOL}=2.6-2.8$. Eyes small, only slightly convergent. Malar space about 0.7 times basal breadth of mandible. Face not protuberant, twice as broad as high. Clypeus 0.65 as wide as face, distinctly divided, punctuate. Tentorial pits indistinct. Mandibles stout, not twisted. Precoxal sulcus narrow. Propodeum convex, rugose and with weak carina; excavate medially. Petiolar tergum 1.5 times longer than wide apically; dorsope distinct; ventral folds of petiolar tergum not joined. Ovipositor very long, 4.5 times length of petiolar tergum. Ovipositor very weakly curved downward, length of ovipositor sheaths 2.0 times petiolar tergum, and 0.95 times fore wing. Hind coxa punctuate or reticulate-punctate; tarsal claws with simple but somewhat thickened at base. Colour black to brown; antennae, head, prothorax and legs yellow-brown; the head is yellowish-brown to orange, except for occiput and frons. Male similar to female except antennae 28 articles, malar space 0.9 times basal width of mandible and length of eye 1.3 times length of temple.

Distribution: $\underline{\text { Japan}}$; $\underline{\text { Sweden. }}$.

Biology: In Japan M. kyushuensis is recorded as a parasitoid of the larvae of the fungus beetle Neotriplax lewisi (Coleoptera: Erotylidae) (Maeto, 1988a). We found 4 specimens within the SMTP and they were caught from July to September in an Alder wood ravine and in a $15 \mathrm{yr}$ old spruce plantation.

\section{Meteorus limbatus Maeto}

Fig. 75, 104

Meteorus limbatus Maeto, 1989:581. Holotype ${ }_{+}$, (Type No. 2655, Kyushu Univ.) Japan: Bonto, Akkeshi, Hokkaido, 10.vii.1977, K. Yamagishi-examined.

Diagnosis: Meteorus limbatus is very similar to M. abscissus in size and structure but the long scattered setae on the clypeus are quite distinct from the dense, short setae of M. abscissus.

Studied material: $\sim 10$ specimens.

Description: Size 3.5-5.0 mm. Antennae with 29-31 articles, all articles longer than wide. Length of eye is more than double the length of temple in dorsal aspect; eyes large, slightly convergent and protuberant; width of face equal its height; $\mathrm{OOL}=1.5$; clypeus equals the width of face, not distinctly divided from face, smoothly arched, tentorial pits distinct; length of malar space 0.75 times basal width of mandible; mandibles slender and twisted. Precoxal sulcus deep and moderately wide. Petiolar tergum apically 1.8-2.3 times longer than wide; dorsope present, laterope deep; ventral folds of petiolar tergum not joined, dorsal aspect with distinct striae; ovipositor straight, thick; length of ovipositor 1.8 times that of petiolar tergum; propodeum with no carina, reticulate and smoothly velved. Fore wing $\mathrm{m}-\mathrm{cu}$ slightly antefurcal; pterostigma brown with lighter boarder. Hind coxa rugose; tarsal claws with a toothed lobe. Colour dark brown with yellow distinct markings. Ocellar area and neck dark. Propodeum, petiolar tergum, precoxal sulcus and mesoscutum dark, scutellum yellow. Dark specimens are known from northern Japan. Male unknown.

Distribution: Palearctic. Country records: France; Italy; Japan; Norway; $\underline{\text { Sweden; United Kingdom. }}$

Biology: We found one specimen within the SMTP and it was caught in July. This specimen was caught in an alder wood ravine. 


\section{Meteorus lionotus Thomson}

Fig. 32, 105

Meteorus lionotus Thomson, 1895:2160. Lectotype §ิ, Sweden: Norrland (MZLU, Lund) - examined.

Meteorus ruficoloratus Fischer, 1957b:4. Holotype $\widehat{\jmath}$, Germany:München, 15.vii.1884. (Kreichbaumer) (ZSBS, Münich).

Diagnosis: The extremely contracted head along with the size of the ocelli make this species easy to distinguish. The only similar species that it can be mistaken for is M. versicolor but the contracted head and the ocellar size $(\mathrm{OOL}=0.5)$ of $M$. lionotus is well distinguished from the more rounded head and smaller ocelli $(\mathrm{OOL}=1)$ of $M$. versicolor.

Studied material: $\sim 10$ specimens.

Description: Size about 4-5mm. Antennae with 31-33 articles, long. Head very strongly contracted behind the eyes. Ocelli very large, $\mathrm{OOL}=0.5$, protuberant. Eyes not strongly protuberant. Clypeus strongly protuberant. Mandibles strongly twisted, small. Precoxal sulcus shallow, rugose. Propodeum generally depressed, with distinct lateral carinae and occasionally with a weak medial transverse carina. Petiolar tergum long with no dorsal pits; ventral borders conjoined at midpoint of segment. Ovipositor about 1.5 times length of petiolar tergum. Legs long, slender; tarsal claws with strong basal lobe. Colour testaceous, black around ocelli and occipital region, legs are always light in colour. Male same as female except that ocelli and eyes slightly smaller.

Distribution: Western Palearctic. Country records: Austria; China; Czechoslovakia; Finland; Germany; Greece; Norway; Poland; Slovakia; $\underline{\text { Sweden; Switzerland; United Kingdom. }}$

Biology: M. lionotus is most commonly recorded as a parasitoid of Geometridae larvae (Huddleston 1980, Yu et al. 2005)

\section{Meteorus longicaudis (Ratzeburg)}

Fig. 106

Perilitus longicaudis Ratzeburg, 1884:55. Syntypes $\odot$, Germany (destroyed).

Diagnosis: Very long ovipositor and transverse head makes this species similar to M. corax and M. nixoni but the number of antennal articles separates M. longicaudis (29-32) from M. corax (39-41) and M. nixoni (43).

Studied material: $\sim 10$ specimens.

Description: Size about $5 \mathrm{~mm}$. Antennal articles 29-32. Head broad, strongly rounded behind eyes. Ocelli small, $\mathrm{OOL}=3$. Eyes slightly convergent. Malar space about equal to basal breadth of mandible. Face not strongly protuberant, about twice as broad as high. Clypeus moderately convex with scattered deep punctures and a broad polished reflexed border. Tentorial pits indistinct. Mandibles stout, slightly twisted. Precoxal sulcus rugose, always broadened anteriorly into a reticulate-rugose area. Propodeum strongly rugose, the carinae strong but not easily seen. Petiolar tergum very broad, length about 1.5 times apical breadth; dorsal pits large, distinct. Ovipositor very long, 4.5 times length of petiolar tergum. Legs long, hind coxa weakly rugose dorsally; tarsal claws simple but somewhat thickened at base, strongly curved. Colour black; antennae, head, prothorax and legs orange-testaceous; the basal half of hind tibia lighter yellow, almost ivory; the head is sometimes infuscated, at least in part, and $3^{\text {rd }}$ abdominal tergite is often lighter in colour than rest of body. Male same as female except antennae longer, up to 36 articles.

Distribution: Western Palearctic. Country records: Croatia; Finland; Germany; Netherlands; Poland; $\underline{\text { Sweden; }}$ Yugoslavia.

Biology: M. longicaudis is recorded as a parasitoid of two beetles, Orchesia micans (Melandryidae) and Eledonoprius amatus (Tenebrionidae) (Huddleston 1980, Yu et al. 2005). 


\section{Meteorus longipilosus Stigenberg sp. nov.}

Fig. 64, 79D, 107

Diagnosis: Meteorus longipilosus at first looks like a hybrid between M. abdominator and M. cespitator. Ovipositor length and head shape is similar to $M$. cespitator while the setae on the gena are long and scattered as in $M$. abdominator. Also the wing venation resembles M. abdominator. The face of M. longipilosus is "meaner looking" (eyes more strongly converging) and the malar space is shorter than in M. abdominator. The two M. longipilosus specimens that we studied differ in size and colour: the paratype is smaller and more brownish than the holotype.

Studied material: Holotype: 1 \& SE. Vb. Vindelns kommun. Kulbäckslidens försökspark. 01.viii18.viii.2003. (leg. SMTP), NHRS-HYME 4977, DNA 144. Paratype:1 $\odot$ SE. Vr. Munkfors kommun. Ransäter, Rudstorp. 23.vii-12.viii.2005. (leg. SMTP), NHRS-HYME 4978, DNA 85.

Description: Body $3.6 \mathrm{~mm}$, fore wing $3.1 \mathrm{~mm}$.

Head: Length of eye 1.4 times length of temple in dorsal aspect; eyes large, convergent, not protuberant; width of face 1.6 times its height; $\mathrm{OOL}=2.5$, ocelli hardly protruding; vertex smooth; clypeus almost as wide as face, distinctly divided from face, rather flat; length of malar space 0.5 times basal width of mandible; mandible stout, not twisted; number of antennal articles 25; 3rd antennal article 4.5 times longer than wide; 4th antennal article 3.6 times longer than wide; penultimate articles as wide as broad or slightly wider.

Mesosoma: Pronotum rugose all the way to the sides. Precoxal sulcus deep and narrow.

Wings: Fore wing: $\mathrm{r} / 3-\mathrm{SR}=0.7$; $\mathrm{C}+\mathrm{SC}+\mathrm{R} / \mathrm{SR} 1=1.4 ; \mathrm{m}-\mathrm{cu}$ antefurcal; pterostigma brown with. Hind wing: $1-$ $\mathrm{M} / \mathrm{cu}-\mathrm{a}=1.1$;

Legs: Hind coxa smooth; hind femur 5.8 times longer than wide; tarsal claws slender with a very small lobe.

Metasoma: Petiolar tergum 1.9 times longer than wide apically, 4.1 times fore wing; dorsope present; ventral borders of petiolar tergum not joined, petiolar tergum striate with no dorsal central rugosity; ovipositor long and weakly curved downward, length of ovipositor 3.1 times petiolar tergum, and 0.75 times fore wing. Propodeum strongly convex, reticulate rugose and with all carina distinct, between carina with weak reticulation.

Colour: Black except: clypeus and palpi yellow; antennae yellow basally to brown apically; petiolar tergum brown and metasoma, legs and hind coxa yellow.

Distribution: Sweden.

Biology: The specimens of Meteorus longipilosus were caught in Malaise traps in late July to August. One was caught in a 15 yrs old spruce plantation and the other was caught on a sandy railway embankment through a pasture.

Etymology: The name longipilosus refers to the long setae that this species has.

\section{Meteorus melanostictus Capron}

Fig. 108

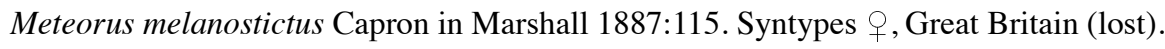

Meteorus niger Lyle, 1913:244. Lectotype , , Great Britain: England, Hampshire, New Forest, 13.v.1911 (Lyle), H. syringaria (BMNH, London) Synonymized by Huddleston, 1980:38.

Meteorus monachae Tobias, 1986:350 Holotype ${ }_{+}^{+}$, Russia: (ZIN. St. Petersburg)-examined. Syn. n.

Diagnosis: Similar to M. cinctellus. Both have a blunt tubercle in front of the fore ocellus but the large ocelli and the presence of a dorsope on the petiolar tergum clearly distinguish M. melanostictus from M. cinctellus, which has small ocelli and no dorsope. M. melanostictus also resembles M. pendulus, which has a dorsope and large ocelli but lacks the blunt tubercle in front of the fore ocellus.

Studied material: 31 specimens.

Description: Size about 4-5 mm. Antennal articles 25-27, all articles at least twice as long as broad. Head strongly contracted behind eyes; a blunt tubercle medially in front of the fore ocellus. Ocelli large, OOL=1.0-1.5. Eyes large, protuberant, moderately convergent. Malar space short, distinctly less than half the basal breadth of mandible. Face about as wide as high, not strongly protuberant. Clypeus strongly protuberant. Tentorial pits deep. Mandibles strongly twisted. Precoxal sulcus thin, foveolate. Propodeum short, with only a strongly raised medial 
transverse carina. Petiolar tergum rather stout with distinct dorsal pits and large glymmae; ventral borders of petiolar tergum widely separated. Ovipositor short, about 1.5 times length of petiolar tergum. Legs short; hind coxa smooth, punctate; tarsal claws with a distinct basal lobe. Colour black; head mostly yellow, prothorax, legs yellow.

Distribution: Western and Eastern Palearctic. Country records: Bulgaria; Germany; Hungary; Ireland; Japan; Korea; Russia; Sweden; Switzerland; United Kingdom.

Biology: M. melanostictus mostly parasitizes on Geometridae but also on Tortricidae (Yu et al. 2005).

\section{Meteorus micropilosus Tobias}

Fig. 109

Meteorus micropilosus Tobias, 1986:350. Russia: 'city Soch, Lazarevskoe, 8-9.V.1975' (Tobias) (ZIN. St. Petersburg) —examined.

Diagnosis: Meteorus micropilosus is similar to M. brevicauda but M. micropilosus has a postfurcal vein m-cu and narrow clypeus, whereas $M$. brevicauda has an antefurcal $\mathrm{m}$-cu vein and a very broad clypeus that almost reaches the eyes.

Studied material: Holotype.

Description: Size about $3 \mathrm{~mm}$. Antennae with 27 articles, all articles at least twice as long as broad. Head rounded behind eyes; a vague punctuated line going from the fore ocellus in between the antennae. Ocelli small, $\mathrm{OOL}=3$. Eyes smaller, protuberant, not convergent. Malar space slightly less than the basal breadth of mandible. Face wider than high, not protuberant. Clypeus slightly protuberant with a few small punctures. Tentorial pits small. Mandibles stout and only slightly twisted. Precoxal sulcus wide, clearly invaginated. Propodeum with all carinae strongly raised, smooth in between. Petiolar tergum rather stout with distinct dorsal pits and large glymmae, noticeably angulate at spiracles; surface rugose centrally turning in to lineate structure laterally; ventral borders of petiolar tergum separated; Ovipositor short, about 1.5-2 times length of petiolar tergum. Hind coxa smoothpunctate; tarsal claws slender. Colour black; clypeus and legs yellow-brown. Pterostigma dark with only small light apical corner. Fore wing vein m-cu postfurcal.

Distribution: Russia.

Biology: The Holotype was caught in the beginning of May in a forest with terrace slopes by the city of Soch, Lazarevskoe in Russia.

\section{Meteorus micropterus (Haliday)}

Fig. 34, 35, 36, 110

Perilitus micropterus Haliday, 1835:27. Lectotype 9 , Ireland:'British, Haliday, 20.2.82' (NMI, Dublin).

Diagnosis: The shape of the head of Meteorus micropilosus is very characteristic by having a flattened frons and a strongly protuberant face, a shelf- shaped face. The only other Meteorus with a similarly shaped head is M. salicorniae but there should be no risk of confusing these species since M. micropterus is small ( $3 \mathrm{~mm}$ ), black and slender with a round head whilst $M$. salicorniae is large $(4-5 \mathrm{~mm})$, reddish brown and with a transverse head.

Studied material: $\sim 150$ specimens.

Description: Size about $3 \mathrm{~mm}$. Antennal articles 23-26, short and thick. Ocelli small, OOL=3. Eyes small, inner orbits parallel; length of malar space at least equal to basal breadth of mandible. Face protuberant, punctate, projecting outward. Clypeus broad, smooth, distinctly divided from the face. Tentorial pits large and deep. Mandibles moderately twisted, large, stout. Precoxal sulcus foveolate. Propodeum reticulate-rugose, without distinct carinae. Petiolar tergum smooth, ventral borders fused beneath for at least the proximal half of the segment. Ovipositor twice length of petiolar tergum, straight. Legs stout; hind coxae rugose dorsally; tarsal claws without a lobe but strongly swollen at base. Wings short, narrow, usually infumate. Colour brownish black. Male same as female except eyes smaller; antennae with 24-28 articles, and articles longer.

Distribution: Palearctic. Country records: Czechoslovakia; Denmark; Finland; Germany; Ireland; Japan; Russia; Slovakia; Sweden; United Kingdom. 
Biology: M. micropterus is a parasitoid of the Lepidoptera family Hepialidae (Huddleston 1980). We found 113 specimens (only 12 were female) within the SMTP. They were caught from July to November but the majority during August and September. They occur in both deciduous and coniferous forests.

\section{Meteorus nixoni Huddleston}

Fig. 76, 111

Meteorus nixoni Huddleston 1980:40. Holotype , , Austria: Tyrol, Oberau, vii.1938 (Nixon) (BMNH, London) —examined.

Diagnosis: Meteorus nixoni is closely related to M. corax and M. sulcatus. All are large and dark species with a wide face. However, M. nixoni has large mandibles that are not twisted and its third abdominal tergum is equipped with only a few striae. M. corax and M. sulcatus both have short, twisted mandibles and they both have the third abdominal tergum densely covered with striae.

Studied material: 4 specimens.

Description: Size up to almost $1 \mathrm{~cm}$. Antennae with 43 articles, long, all articles at least longer than broad. Head broad, contracted behind eyes but not strongly so; temples shorter than eyes. Ocelli small, OOL $=2.5$ times OD. Eyes small, protuberant, not or very little convergent. Malar space slightly shorter than basal breadth of mandible. Face about twice as broad as high, not strongly protuberant, centrally reticulate-rugose, laterally reticulatepunctate. Clypeus broad, only slightly narrower than face, not strongly protuberant but deeply impressed at the junction with face and with a strongly reflexed apical margin, sparsely punctured; clypeal hair conspicuously longer than facial hair. Tentorial pits deep and large. Mandibles large, not twisted. Pronotum strongly rugose. Mesonotum rather narrow, densely almost reticulately punctate, especially on the central lobe; notaulices thin, deeply impressed. Precoxal sulcus reticulate- rugose; rest of mesopleurae polished, punctate except for a subalar patch of rugosity. Propodeum with basal and medial transverse carinae and a central longitudinal carina, with rugose sculpture between. Petiolar tergum rather broad at base, apically only 2.5 times as broad as at narrowest point, the spiracles distinctly before mid-point; dorsal pits large and deep, behind dorsal pits strongly longitudinally strigose. $3^{\text {rd }}$ abdominal tergum with distinct thyridia and a trace of longitudinal strigose sculpture. Ovipositor long, about 4 times length of petiolar tergum. Legs long; hind coxa shining, punctured but not densely so; hind femur densely reticulate-punctate; hind tibia swollen, though not as broad as femur, and narrow at base; tarsal claws thick, strongly curved and strongly swollen at base, almost with a basal lobe. Wings large, infumate. Colour: dark reddish-brown; mandibles, palps, fore and mid legs testaceous, hind coxa and femur brown, tibia black except at the base which is light yellow, hind tarsi black except at base and apex which are light yellow; malar space and clypeus slightly lighter in colour than rest of head.

Distribution: Palearctic. Country records: Austria; Japan; Korea; Russia.

Biology: The holotype was caught in July.

\section{Meteorus obfuscatus (Nees von Esenbeck)}

Fig. 66, 112

Bracon obfuscatus Nees von Esenbeck, 1811:22. Syntypes, Germany (destroyed).

Zele thoracicus Curtis, 1832: folio 415. Syntypes, Great Britain: England, Regent's Park (NMV, Melbourne) synonymized by Haliday, 1835:31.

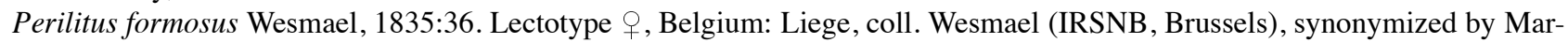
shall, 1887:106.

Meteorus fodori Papp, 1973:3 Holotype , Yugoslavia: Montenegro, Bjela Gora, Grahovo, 1929 (Fodor) (HMHM, Budapest), synonymized by Huddleston, 1981:41. Male from same coll examined

Diagnosis: Meteorus obfuscatus is similar to M. cespitator and to M. hirsutipes but has a much shorter ovipositor (1.7-2.0 times petiolar tergum). The ovipositor is 3-4 times the petiolar tergum in M.cespitator and 2.2-2.5 times the petiolar tergum in M. hirsutipes. 
Studied material: $\sim 50$ specimens.

Description: Size about 4-5mm. Antennae short, 23-29 articles, the basal articles about twice as long as broad, often shorter; the apical half are as long as broad. Head behind eyes contracted, rounded. Ocelli small, $\mathrm{OOL}=2.5$. Eyes large, protuberant, slightly convergent. Malar space short, half the basal breadth of mandible. Face 1.5 times as broad as high, not strongly protuberant. Clypeus protuberant, slightly narrower than face sometimes with an impression in the medial part of the apical border. Tentorial pits small. Mandibles stout, moderately twisted. Precoxal sulcus deep and foveolate. Propodeum with distinct carinae, rugae in between. Petiolar tergum stout with distinct dorsal pits; generally somewhat raised medially. Ovipositor twice the length of petiolar tergum, slender, usually straight but in some down-curved. Legs long, slender; hind coxa smooth punctate; tarsal claws long, without a basal lobe but slightly swollen at base. Colour brownish black, legs always more or less yellow, prothorax usually orange-testaceous as well as sometimes antennae, head, mesothorax and abdomen. Male same as female, antennae longer, 29-32 articles.

Distribution: Palearctic. Country records: Belgium; Czechoslovakia; Finland; France; Germany; Hungary; Italy; Japan; Poland; Romania; Russia; Slovakia; Sweden; Switzerland; United Kingdom; Yugoslavia.

Biology: We found 3 specimens of M. obfuscatus within the SMTP. They were caught during August and September in an old heath with old beeches and in an old aspen forest. Host records of M. obfuscatus include five different coleopteran families: Cerambycidae, Erotylidae, Melandryidae (Orchesia micans) and Tenebrionidae (Huddleston 1980, Yu et al. 2005).

\section{Meteorus obsoletus (Wesmael)}

Fig. 113

Perilitus obsoletus Wesmael, 1835:49. Lectotype + , Belgium: Brussels, coll Wesmael (IRSNB, Brussels).

Meteorus viridane Johansson, 1964:251. Holotype , Sweden: Borgholm, 30.vi.1954, ex larva of Tortrix viridanae (L.) coll. 13 -16.vi.1954 (Johansson) (MZLU, Lund) synonymized by Huddleston, 1980:42 —examined

Diagnosis: Meteorus obsoletus is very similar to M. versicolor. The best diagnostic characters to separate these species are the length and shape of the ovipositor (slender and long, 2.0-2.5 times petiolar tergum in the former; stouter, 1.5-2.0 times petiolar tergum in the latter), the shape of the eyes (not so protruding in the former, strongly protruding in the latter) and the temple (longer and more rounded in the former, more contracted in the latter).

Studied material: $\sim 10$ specimens.

Description: Size about 3.5-4mm. Antennal articles 27-30. Head rounded behind eyes. Ocelli large, $\mathrm{OOL}=\mathrm{OD}$. Eyes not protuberant, slightly convergent. Clypeus slightly protuberant. Tentorial pits small and shallow. Mandibles small, strongly twisted. Precoxal sulcus reticulate-rugose. Propodeum rounded with a weak medial impression posteriorly, irregularly reticulate-rugose. Petiolar tergum slender, finely longitudinally striate dorsally, without dorsal pits. Ovipositor 2.0-2.5 times length of petiolar tergum, straight and slender. Hind coxa smooth, tarsal claws with strong basal lobe. Colour brownish black; head, prothorax, legs, mesothorax and abdomen usually testaceous. Male same as female except eyes and ocelli smaller.

Distribution: Palearctic. Country records: Austria; Belgium; Bulgaria; Czechoslovakia; France; Germany; Ireland; Italy; Japan; Korea; Moldova; Netherlands; Sweden; Switzerland; Turkey; Ukraine; United Kingdom; Yugoslavia.

Biology: We found one specimen within the SMTP. It was caught in the middle of July in an old spruce forest. Host records of M. obsoletus include four different Lepidoptera families, but the predominant hosts appear to belong to the family Tortricidae. In Japan they were found on the species Choristoneura diversana (Tortricidae) and Huddleston (1980) also mentions the species Tortrix viridana and Gypsonoma dealbana (Tortricidae).

\section{Meteorus oculatus Ruthe}

Fig. 114

Meteorus oculatus Ruthe, 1862:23. Lectotype + , Germany: Ruthe coll. (BMNH, London) - examined. 
Meteorus pachypus Schmiedeknecht, 1897:207. Lectotype $\odot$, Germany: coll. Schmiedeknecht (ZMHB, Berlin), synonymized by Huddleston, 1980:42.

Diagnosis: The eyes of Meteorus oculatus are very large, protruding and strongy converging, a unique combination of characters among western Palearctic species. The Asian species (Japan, Korea and China) M. graciliventris and M. endoclytae also have large, protruding and converging eyes but they are much larger species with body length up to $7 \mathrm{~mm}$.

Studied material: $\sim 40$ specimens.

Description: Size about 4-5mm. Antennal articles 30-33, rather slender, in apical half of the flagellum, the articles are only slightly longer than broad. Head broad, strongly contracted behind eyes. Ocelli small, OOL about 2.5. Eyes very large, protuberant, strongly convergent. Malar space very short, much less than half the basal breadth of a mandible. Face about as broad as high. Clypeus slightly protuberant. Tentorial pits large. Mandibles slightly twisted. Precoxal sulcus deep. Propodeum rather narrow, strongly rugose with indistinct carinae. Petiolar tergum long, slender; dorsal pits distinct but small. Ovipositor long and straight, about 3 times length of petiolar tergum. Hind tibia strongly swollen, about as thick as femur but constricted at base. Tarsal claws short, simple and thick, strongly expanded at base. Colour black, legs testaceous; antennae, clypeus, mandibles, pronotum ventrally and $3^{\text {rd }}$ abdominal tergite also lighter in colour; wings infumate. Male same as female except that eyes are less strongly convergent and hind tibia not strongly swollen.

Distribution: Western Palearctic. Country records: Austria; Bulgaria; Czechoslovakia; Finland; Germany; Hungary; Kyrgyzstan; Lithuania; Norway; Poland; Sweden; Switzerland; Turkey; United Kingdom.

Biology: We found 29 specimens within the SMTP. They were caught from end of June to the end of September in mixed forests or in open terrain. M. oculatus is only recorded from Taleporia tubulosa (Lepidoptera Psychidae) (Yu et al. 2005).

\section{Meteorus pendulus (Müller, 1776)}

Fig. 12, 22, 50, 72, 116

Meteorus pendulus Müller, 1776:106

Meteorus pendulator Latreille, 1799:138. M. pendulator is an invalid emendation of M. pendulus Müller, 1776 (Yu et al., 2005)

Ichneumon gyrator Thunberg, 1822:261. Holotype $\hat{\jmath}$, Sweden (UDE, Uppsala) synonymized by van Achterberg et al. 2009:790-examined.

Perilitus scutellator Nees von Esenbeck, 1834:38. Syntypes, Germany (lost), synonymized by Roman, 1912:289.

Bracon petiolator Zetterstedt, 1838:399. Lectotype, Sweden: Scandinavia Lapponia. (MZLU, Lund) synonymized by Papp, 1994:306-examined

Meteorus melanostictus Capron in Marshall, 1887:115. Males only from type-series.

Meteorus parvulus Thomson, 1895:2156. Holotype + , Sweden: Öland (MZLU, Lund) synonymized by Huddleston, 1980:32-examined.

Diagnosis: Meteorus pendulus resembles M. ictericus but there are some good characters separating them. M. ictericus has whitish hairs between the eyes and the clypeus, rugose glymmae, and a long ovipositor (2.5-3.0 times length of petiolar tergum). M. pendulus lacks the whitish hairs on the face, the glymmae are smoother, and the ovipositor is short and stout (only 1.5-2.0 times petiolar tergum).

Studied material: $\sim 60$ specimens.

Description: Size about 4.5-5mm. Antennae long, 30-34 articles. Head always contracted behind eyes, occasionally strongly rounded. Frons with a depression in front of the fore ocellus. Ocelli large, OOL $=1.0-1.5$ (seldom $\mathrm{OOL}=2$ ). Eyes large, protuberant, slightly convergent. Malar space equal or a little less than basal breadth of mandible. Face little broader than high, slightly protuberant. Clypeus strongly protuberant. Mandibles long, strongly twisted. Precoxal sulcus narrow, foveolate. Propodeum broad, rather flat, with dense, fine rugosity, with a central longitudinal carina. Petiolar tergum with dorsal pits, shallow glymmae. Ovipositor short, 1.5-2.0 times length of petiolar tergum, thick. Legs long, thin; hind coxa smooth, punctate, often laterally with a trace of rugosity. Tarsal claws with a basal lobe. The pterostigma always uniformly pale testaceous. Forewing vein m-cu mostly interstitial 
but in some specimen postfurcal. Colour mostly orange-testaceous with sometimes some black areas. Male same as female except eyes smaller and malar space longer; colour darker, the pterostigma often dark with a pale border.

Distribution: Western and Eastern Palearctic. Country records: Austria; Azerbaijan; Belgium; Bulgaria; China; Croatia; Cyprus; Czechoslovakia; Denmark; Egypt; Finland; France; Germany; Greece; Hungary; Iran; Ireland; Israel; Italy; Japan; Korea; Latvia; Lithuania; Madeira Islands; Moldova; Mongolia; Netherlands; Norway; Poland; Romania; Russia; Slovakia; Sweden; Switzerland; Turkey; Ukraine; United Kingdom; Yugoslavia.

Biology: We found 29 specimens within the SMTP. They were mostly caught during July and August in a wide variety of biotopes with a slight preference towards pine forests. Host records of $M$. pendulus include the lepidopteran families Geometridae, Lasiocampidae, Lycaenidae, Lymantriidae, Noctuidae and Tortricidae (Huddleston 1980, Yu et al. 2005).

\section{Meteorus politutele Shenefelt}

Fig. 59, 117

Meteorus politutele Shenefelt, 1969:86.

Meteorus politus Telenga, 1950

Diagnosis: This is a large, punctate, yellow species with two dark spots on the petiolar tergum, a unique combination of characters in the genus.

Studied material: 1 specimen.

Description: Size 4-5mm. Antennal articles about 30, all longer than broad, basal articles darker in colour. Head broad, strongly contracted behind eyes. OOL=1.5. Frons with rather deep ventrally striated invagination. Eyes large, protuberant and only slightly converging. Malar space equal to mandible base or slightly shorter. Face 1.6 broader than high, not protruding. Clypeus protuberant, mandibles somewhat smaller and slightly twisted. Precoxal sulcus broad, whole area strongly punctuate. Propodeum broad and with no distinct carina. Petiolar tergum with dorsope, dorsal surface finely striated. Petiolar tergum 3.7 times fore wing. Hind coxae slightly punctuate.

Distribution: Turkey; Uzbekistan.

Biology: The male lectotype was caught in Uzbekistan, in May 1929.

\section{Meteorus pulchricornis (Wesmael)}

Fig. 23, 28, 50, 72, 118

Perilitus pulshricornis Wesmael, 1835:42. Lectotype ${ }_{+}$, Belgium: Brussels, coll. Wesmael (IRSBN, Brussels).

Meteorus striatus Thomson, 1885:2157. Lectotype +, Sweden: Skåne, Pålsjö (MZLU, Lund) synonymized by Huddleston, 1980:45-examined.

Meteorus thomsoni Marshall, 1899: 301.

Meteorus japonicus Ashmead, 1906:190. Lectotype ${ }_{+}$, Japan:Gifu, viii.1902 (USNM, Washington), synonymized by Marsh (1979).

Meteorus nipponensis Viereck, 1912:624.Holotype $\odot$, Japan (USNM, Washington) synonymized by Watanabe, 1939

Meteorus macedonicus Fischer, 1957a:104. Holotype , Yugoslavia: Macedonia, treskaslucht (NHM, Vienna), synonymized by Huddleston, 1980:45

Meteorus graeffei Fischer, 1957a:107. Holotype $\odot$, Italy:'Triest' (NHM, Vienna), synonymized with macedonicus by Fischer, 1970b:287.

Meteorus tuberculifer Fischer, 1957a:108. Holotype , Italy:'Trieste Küstenland, coll Graeffe (NHM, Vienna), synonymized by Huddleston, 1980:45.

Meteorus baicalensis Telenga, 1950, synonymized by Belokobylskij 2000:209.

Diagnosis: The dense, short and erect setae on the clypeus along with the indications of dorsope on the petiolar tergum are good characters for distinguishing M. pulchricornis. It is closest to, and easily confused with M. abscissus. M. abscissus has the ventral sides of the petiolar tergum not closed, contrary to M. pulchricornis which have the ventral sides of the petiolar tergum closed. The dorsal pits on the petiolar tergum of M. abscissus are sometimes small and complicates the identification with M. pulchrichornis that has no true dorsal pits. 
Studied material: $\sim 80$ specimens.

Description: Size about 5mm. Antennae with 29-33 articles, long, slender; all articles distinctly longer than broad. Ocelli large, $\mathrm{OOL}=1.5$. Eyes large, protuberant, moderately convergent. Malar space slightly shorter than basal breadth of mandible. Face not strongly protuberant but slightly raised medially. Clypeus strongly protuberant, evenly convex with a dense pile of erect setae. Mandibles small and strongly twisted. Precoxal sulcus reticulaterugose with emphasis on reticulate. Propodeum without distinct carinae, strongly reticulate-rugose. Petiolar tergum longitudinally striate, usually with no dorsal pits but with indications of pits. Ovipositor 1.5-2.0 times length of petiolar tergum. Legs long, slender; hind coxa generally completely rugose, this sculpture always fine, never reticulate though sometimes transverse. Tarsal claws with a strong basal lobe. Colour mostly yellow. Completely pale specimens sometimes occur. Male same as female except antennae slightly longer; face occasionally strongly raised medially; propodeum with greater variation in sculpture, smaller, and more depressed.

Distribution: Palearctic, Oceanic and Nearctic. Country records: Armenia; Austria; Azerbaijan; Belarus; Belgium; Bulgaria; Chile; China; Croatia; Cyprus; Czechoslovakia; Finland; France; Georgia; Germany; Greece; Hungary; India; Iran; Ireland; Israel; Italy; Japan; Korea; Lithuania; Macedonia; Moldova; Morocco; Netherlands; New Zeeland; Norway; Poland; Portugal; Romania; Russia; Slovenia; Spain; Sweden; Switzerland; Turkey; Ukraine; United Kingdom; USA; Yugoslavia.

Biology: We found two specimens within the SMTP. They were caught in September, one in a mixed forest and the other in a heather heath. M. pulchricornis is primarily a parasitoid of Noctuidae, although there are records from 17 other lepidopteran families (Yu et al. 2005). Amongst the studied Meteorus material at Hokkaido University in Japan, we found specimens that was reared from Evergestis forficalis, Leucania separate, Zanclognatha fractalis, and Ilema sorocula. The biology and ecology of this species have been studied extensively (Askari et al. 1977, 1978, Fuester et al. 1993, Berry et al. 2004, Chau et al. 2009).

\section{Meteorus punctifrons Thomson}

Fig. 55, 119

Meteorus punctifrons Thomson, 1895:2166. Holotype ${ }_{+}$, Sweden: Åreskutan i Jämtland (MZLU, Lund) - examined.

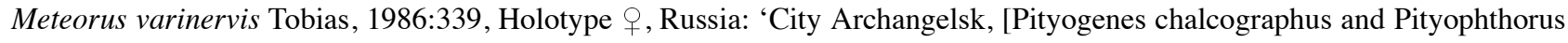
micrographus] 6.VII.1964’ (Pryakhina), (ZIN. St. Petersburg)—examined. Syn. n.

Diagnosis: This is a small, slender species, and it is rare, at least in collections. It is easily recognized by the characteristic frontal punctuation, the subcubic head and the minute ocelli. Similar small species are M. brevicauda and M. micropilosus, but neither of them has a subcubic head with large frontal punctures.

Studied specimens: $\sim 10$ specimens.

Description: Size small. Antennal articles 26-27. Head subcubic. Ocelli very small OOL=4 times OD. Eyes small, only slightly convergent. Face about twice as broad as high; frons with a short groove in front of ocellus. There is a patch, between ocelli and eye, of large punctures separated at most by a distance equal to their diameter. Mandible stout, slightly twisted. Propodeum short, with distinct carinae, excavate apically. Petiolar tergum short and wide, with small but distinct dorsal pits. Ovipositor short, about 15 times length of petiolar tergum, downcurved. Legs very long, slender; hind coxa only slightly rugose at the base; tarsal claws long, slightly swollen at base. Colour brownish black with testaceous parts. Male same as female.

Distribution: Western Palearctic. Country records: France; Norway; Russia; Sweden.

Biology: Meteorus punctifrons is a parasitoid of Corticeus longulus (Coleoptera, Tenebrionidae) (Huddleston, 1980).

\section{Meteorus radialis Tobias}

Fig. 56, 120

Meteorus radialis Tobias, 1986:341. Holotype ${ }_{+}$, Russia: (ZIN. St. Petersburg)-examined. 
Diagnosis: The radial cell, small ocelli and long ovipositor are striking features. No other European Meteorus species has a wing venation like Meteorus radialis.

\section{Studied material: Holotype.}

Description: Size medium to small. Antennal articles 24. Head rounded behind the eyes. Ocelli very small, OOL up to 6 times OD. Eyes large, protruding and converging. Malar space 0.6 times mandible base. Mandibles stout not twisted. Face 1.4 times broader than high, not protruding but medially rugose. Clypeus protruding, with long setae and as wide as face, punctate. Precoxal sulcus very broad, punctuate, about $90 \%$ of mesopecta reticulatepunctate. Propodeum reticulate - rugose and with longitudinal and transverse carina. Petiolar tergum with dorsal pits and dorsal striate. Fore wing 4.2 times petiolar tergum. Ovipositor long, 2.3 times petiolar tergum. Hind coxae rugose. Radial cell of forewing very narrow, stigma narrow and brown.

Distribution: Kola peninsula, Russia.

Biology: Both the holotype and the paratype were caught in July in a forest. Otherwise the biology is unknown.

\section{Meteorus rubens (Nees von Esenbeck)}

Fig. 19, 25, 27, 29, 121

Bracon rubens Nees von Esenbeck, 1811:22. Syntypes 9 , Germany lost

Perilitus leviventris Wesmael, 1835:46. Lectotype + , Belgium: Brussels, coll. Wesmael (IRSNB, Brussels), synonymized by Fischer, 1970b:260 - examined.

Meteorus islandicus Ruthe, 1859.317. Syntypes, Iceland: Staudinger coll. (NM, Vienna), synonymized by Roman, 1917:4examined.

Meteorus medianus Ruthe, 1862:53. Lectotype + , Germany: Ruthe coll. (BMNH, London), synonymized with leviventris by Marshall, 1887:126-examined.

Meteorus scutatus Costa, 1884:172. Holotype + , Italy: Oristano (MZ, Naples), synonymized by Huddleston, 1980:47

Meteorus heteroneurus Thomson, 1895:2158. Holotype , , Sweden:Västergötland (MZLU, Lund), synonymized by Fischer, 1970b:260-examined.

Meteorus szechuanensis Fahringer, 1935:11. Lectotype ${ }_{+}$, China: No Szechuan (NHRS, Stockholm), synonymized by Huddleston, 1980:47-examined.

Meteorus mesopotamicus Fischer, 1957a:105. Holotype + , Iraq: Mosul (NM, Vienna), synonymized by Huddleston, 1980:47— examined.

Diagnosis: The traces of dorsopes on the petiolar tergum, the shortened marginal cell and the ovipositor are key characters for Meteorus rubens. The closest species to M.rubens is M. heliophilus, but the longer marginal cell of the latter and the more numerous antennal articles (30-32 in M. heliophilus, 24-28 in M. rubens) are good characters to separate these species.

Studied material: $\sim 100$ specimens.

Description: Size about 4-5mm. Antennae short, 24-28 articles; the articles in the distal half of the flagellum often only as long as broad but sometimes longer than this. Ocelli large, $\mathrm{OO}=1.0-1.5$. Eyes only slightly convergent. Malar space slightly less than basal breadth of mandible. Face a little less than twice as wide as high, protuberant with a medial longitudinal raised area which is finely transversely rugose. Clypeus protuberant, transverse with long setae. Tentorial pits large, distinct. Mandibles long, moderately twisted, the upper tooth generally rather long. Precoxal sulcus a shallow rugose furrow. Propodeum rugose and generally with only a medial longitudinal carina. Petiolar tergum often with traces of dorsal pits. Ventral borders of petiolar tergum conjoined in the midpart of the segment. Ovipositor twice length of petiolar tergum, thick, strongly swollen at the base. Hind coxa smooth, punctuate-weak rugosity dorsally at the base. Tarsal claws never strongly bent and usually only swollen at the base. Colour varies from completely black to completely yellow with all intermediate stages represented. Male same as female except antennae longer, 26-30 articles, all flagellar articles distinctly longer than broad; the propodeum is more depressed, often less strongly rugose and with the carinae more distinct.

Distribution: Palearctic and Nearctic. Country records: Algeria; Argentina; Armenia; Austria; Azerbaijan; Belgium; Bulgaria; Canada; China; Costa Rica; Croatia; Cyprus; Czechoslovakia; Denmark; Egypt; Faeroe Islands; Finland; France; Germany; Greece; Greenland; Hungary; Iceland; Iran; Iraq; Ireland; Israel; Italy; Japan; Kazakhstan; Korea; Latvia; Lithuania; Moldova; Mongolia; Netherlands; Norway; Poland; Romania; Russia; Slovakia; Slovenia; Spain; Sweden; Switzerland; Tunisia; Turkey; Turkmenistan; USA; Ukraine; United Kingdom; Yugoslavia. 
Biology: We found 35 specimens within the SMTP. They were caught from July to September in both deciduous and coniferous forests. Meteorus rubens has been recorded as a parasitoid of several families of Lepidoptera, but members of the Noctuidae appear to be the primary hosts (Yu et al. 2005).

\section{Meteorus ruficeps (Nees)}

Fig. 122

Meteorus ruficeps Nees, 1834:39. Type material lost. Synonymized by Papp 1983:318.

Perilitus pallipes Wesmael. 1835:29. Lectotype 옹 Belgium: coll. Wesmael (IRSNB, Brussels). Synonymized by Papp 1983:318.

Meteorus nigritarsis Ruthe, 1862:21. Holotype , Germany: Ruthe coll. (BMNH, London), synonymized with pallipes by Huddleston, 1980:43 - examined.

Diagnosis: Meteorus ruficeps and M. ictericus are quite similar morphologically. The main differences between them involve the size of the ocelli and the convergence of the eyes. Meteorus ruficeps has converging eyes, the face is higher than broad, and the ocelli are slightly smaller $(\mathrm{OOL}=1.5-2.0)$. The eyes of M. ictericus are not so converging, the face is as wide as high, and the ocelli are larger $(\mathrm{OOL}=1.0-1.5)$.

Studied material: $\sim 30$ specimens.

Description: Size about 4-5mm. Antennal articles 26-31, all articles slightly longer than broad. Head strongly rounded behind eyes. Ocelli large, $\mathrm{OOL}=1.5-2$ times OD. Eyes strongly convergent. Malar space short, about half basal breadth of mandible. Face with a slightly raised medial longitudinal area. Tentorial pits deep. Mandibles stout, moderately twisted. Precoxal sulcus foveolate. Propodeum rugose with usually only the medial transverse carina developed. Petiolar tergum stout with distinct dorsal pits and glymmae. Ovipositor 2.5-3.0 times length of petiolar tergum. Hind coxa smooth, punctate; tarsal claws with distinct basal lobe. Colour black, legs always yellow; head at least in part yellow. Male same as female except antennae longer, eyes not strongly convergent.

Distribution: Western Palearctic. Country records: Austria; Bulgaria; Czechoslovakia; France; Germany; Hungary; Japan, Latvia; Macedonia; Norway; Poland; Slovakia; Sweden; Switzerland; United Kingdom; Yugoslavia.

Biology: We found 11 specimens within the SMTP. They were caught from July to September, mostly in coniferous forests. M. ruficeps is primarily a parasitoid of Tortricidae (Lepidoptera) but it has also been recorded as attacking Gelechiidae, Geometridae, Noctuidae and Tineidae (Huddleston 1980, Yu et al. 2005).

\section{Meteorus rufus (De Geer)}

Fig. 123

Ichneumon rufus De Geer, 1778:596. Type material lost.

Perilitus unicolor Wesmael, 1835:41. Lectotype ㅇ, Belgium: Brussels, coll. Wesmael (IRSNB, Brussels) - examined.

Saprotichus chinensis Holmgren, 1868:430. Holotype ${ }_{+}$, China (NHRS, Stockholm), synonymized by Huddleston, 1980:51 examined.

Diagnosis: Meteorus rufus is similar to M.pendulus but it has longer antennae with more antennal articles (35-36 articles in the former, 30-34 in the latter) and larger ocelli ( $\mathrm{OOL}=2$ in the former, $\mathrm{OOL}=1-1.5$ in the latter).

Studied material: $\sim 10$ specimens.

Description: Size about $5.5 \mathrm{~mm}$. Antennae long, up to 36 articles; all articles of flagellum distinctly longer than broad, most at least twice as long as broad. Ocelli large, $\mathrm{OOL}=2$ times, protuberant. Eyes protuberant, slightly convergent. Malar space about equal in length to basal breadth of mandible. Face 1.5-2.0 times as broad as high, not strongly protuberant but distinctly raised medially and there with transverse structure. Clypeus strongly protuberant, densely rugulose-punctate with scattered long setae. Mandibles long, slender, strongly twisted. Precoxal sulcus broadened, strongly reticulate-rugose. Propodeum broad and with no distinct carinae dorsally. Petiolar tergum stout with large dorsal pits and distinct glymmae; ventral borders of petiolar tergum only narrowly separated at mid point of segment; dorsal surface longitudinally striate. Ovipositor twice the length of petiolar tergum, thick, 
strongly swollen at base, straight. Hind coxa foveolate laterally, often with a few strong rugae dorsally. Tarsal claws with a large basal lobe. Colour testaceous, base of petiolar tergum paler yellow.

Distribution: Western Palearctic. Country records: Austria; Belgium; Croatia; Cyprus; Czechoslovakia; France; Germany; Hungary; India; Ireland; Israel; Italy; Poland; Romania; Sweden; Switzerland; Turkey; Ukraine; United Kingdom; Yugoslavia.

Biology: Meteorus rufus is recorded in the literature as a parasitoid of seven different lepidopteran families: Geometridae, Lymantriidae, Noctuidae, Pieridae, Pyralidae, Tortricidae and Zygaenidae (Huddleston 1980, Yu et al. 2005). The specimens we studied were all associated with Zygaenidae when host data were available.

Remarks: De Geer collected a specimen at his estate in Sweden that he described in 1778 as Ichneumon rufus. According to the German Braconidae checklist by Belokobylskij et al. (2003), Ichneumon rufus De Geer, 1778 is a synonym for M. unicolor. We have studied the type material of M. unicolor (lectotype) from Belgium and conclude that it agrees with our interpretation of the description of M. rufus. We have also studied the De Geer collection but, as Huddleston wrote in his revision in 1980, there is no specimen of Ichneumon rufus present and no pinhole in the cork below the label. The specimen has probably been lost prior to the relocation of the De Geer specimens into new drawers.

\section{Meteorus salicorniae Schmiedeknecht}

Fig. 65, 124

Meteorus salicorniae Schmiedeknecht, 1897:189. Lectotype ㅇ, Germany: Artern (ZMHB, Berlin).

Meteorus ocellatus Watanabe, 1951:45. Holotype , Japan: Kyushu Hikosan, 17.v.1948 (Yasumatsu) (EI, Sapporo), synonymized by Huddleston, 1980:48.

Diagnosis: Meteorus salicorniae has four characteristics of the head that are very distinctive: the protruding face, the depression in the frons, the transverse face and the shape of the antennae. The only species in the Western Palearctic with a similarly protruding face is $M$. micropterus, but M. micropterus has a subcubic face, not transverse, and the antennae are only slightly tapering to the apex, not strongly so as in M. salicorniae.

Studied material: $\sim 10$ specimens.

Description: Size $4-5 \mathrm{~mm}$. Antennal articles $33-35$. The articles are thick at base and strongly tapering to apex. The antennae are set in a depression in the frons. Ocelli small, $\mathrm{OO}=2.5-3.5$. Eyes small and not convergent. Face protuberant. Malar space slightly longer than the basal breadth of mandible. Face transverse, about twice as broad as high, minutely punctured. Clypeus protuberant and not as broad as face. Mandibles large, not twisted. Precoxal sulcus very deep. Propodeum high, rounded with diffuse carinae though strongly reticulate-rugose. Petiolar tergum with small but distinct dorsal pits. Ovipositor about 2.5 times the length of petiolar tergum. The ovipositor sheaths with erect long setae. Colour testaceous.

Distribution: Western and Eastern Palearctic. Country records: Austria; Bulgaria; Croatia; Czechoslovakia; Germany; Greece; Hungary; Italy; Japan; Korea; Romania; Switzerland; Turkey; Yugoslavia.

Biology: M. salicorniae is recorded as a parasitoid of the lepidopteran family Pyralidae (Yu et al. 2005).

\section{Meteorus sibyllae Stigenberg sp nov.}

Fig. 79E, 125

Diagnosis: Meteorus sibyllae is most similar to M. cespitator and M. jaculator. It is most easily separated from $M$. cespitator on the body size. Whilst $M$. sibyllae belongs to the smaller species in the genus (length $<3.5 \mathrm{~mm}$ ), $M$. cespitator is distinctly larger (length $>3.5 \mathrm{~mm}$ ). The face of $M$. sibyllae is also more transverse and wider than that of $M$. cespitator (face 1.8 times as wide as high in the former, 1.5 times in the latter). M. jaculator is as small as $M$. sibyllae but has fewer antennal articles (18-22 in M. jaculator, 24-26 in M. sibyllae).

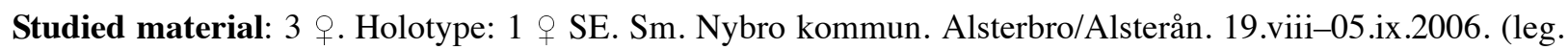
SMTP), NHRS-HYME 5208, DNA 172. Paratype: 2 \& SE. Dr. Säters kommun. Säterdalen, Näsåkerspussen. 21.vii-04.viii.2003. (leg. SMTP), NHRS-HYME 5220, NHRS-HYME 5218 is mounted for SEM. 
Description: Body $3.0 \mathrm{~mm}$, forewing $3.2 \mathrm{~mm}$ of holotype. Paratypes have $2.7-3.0 \mathrm{~mm}$ in body lengths. Head: Length of eye equals the length of temple in dorsal aspect; eyes small, not convergent, slightly protuberant, smooth with punctation; width of face 1.8 times its height; $\mathrm{OOL}=3-3.5$; clypeus distinctly shorter than the width of face, distinctly divided from face, convex, tentorial pits distinct; length of malar space 0.6 times basal width of mandible; mandibles stout and not twisted; antennal articles 24-26; 3rd antennal article 3.5 times longer than wide; all articles longer than wide, subapical articles 1.5 times longer than wide.

Mesosoma: Precoxal sulcus deep and narrow.

Wings: Fore wing: $\mathrm{r} / 3-\mathrm{SR}=0.4 ; \mathrm{C}+\mathrm{SC}+\mathrm{R} / \mathrm{SR} 1=1.37 ; \mathrm{m}-\mathrm{cu}$ antefurcal; pterostigma brown with apical corner light. Hind wing: $1-\mathrm{M} / \mathrm{cu}-\mathrm{a}=1.2$;

Legs: Hind coxa rugose dorsally; hind femur 4.8 times longer than wide; tarsal claws small with no lobe.

Metasoma: Petiolar tergum apically 1.5 times longer than wide, 4.2 times as long as forewing; dorsope present; ventral folds of petiolar tergum not joined, dorsal aspect with no ridges, evenly curved with weak rugosity medially and striae laterally; ovipositor straight, slender; length of ovipositor 3.5-4.0 times that of petiolar tergum and 0.75 times forewing; propodeum with vague carina and reticulation.

Colour: Dark brown - black, clypeus yellow, legs yellow - brown, coxae dark brown.

Distribution: Sweden.

Biology: We found 3 specimens within the SMTP. They were caught in August in deciduous and mixed forests. Etymology: This species is named after Ms Sibyllae Noack, a fellow PhD student.

\section{Meteorus stenomastax Stigenberg sp. nov.}

Fig. 38, 39, 46, 79F, 126

Diagnosis: The body has a unique colour combination of light red and darker red-brown patches and that in combination with the slender antennae and long, yellow-brown coloured pterostigma distinguish Meteorus stenomastax from related species. In Huddleston's key it runs to M. cinctellus but the long and slender antennae and ovipositor length (length of ovipositor 1.8 times petiolar tergum in the former and 2.0-2.5 in the latter) distinguish it from $M$. cinctellus, which has rather stout antennae.

Studied material: 6 ๆ. Holotype: 1 \& SE. Vb. Vindelns kommun. Kulbäckslidens försökspark. 01.ix-

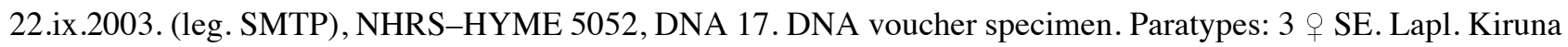
kommun. Abisko national park. 08.vii-05.viii.2004. One dry-pinned (left hind leg damaged and glued separately) NHRS-HYME 5051, and one in ethanol DNA 16, and the third is a mounted SEM specimen and DNA 119 (leg. SMTP), in NHRS. SE. Sö. Huddinge kommun. Sofielunds återvinningsanläggning. 16.vi.30.vi.2004. (leg. SMTP), NHRS-HYME 5213, DNA 173. 1 Dalarna, (UUZM).

Description: Body $4.4 \mathrm{~mm}$, fore wing $3.7 \mathrm{~mm}$.

Head: Length of eye 1.6 times length of temple in dorsal aspect; eyes large, convergent and protuberant; face as wide as high; $\mathrm{OOL}=1.9$; vertex smooth; frons with a prominent tubercle; clypeus as wide as minimum width of face, protruding and distinctly divided from face, convex with large punctuation; tentorial pits distinct; length of malar space 0.5 times basal width of mandible; mandibles twisted; antennal articles 28 ; 3rd antennal article 3.5 times longer than wide and 4th antennal article 3.25 times longer than wide; all articles longer than wide, subapically at least 1.5 times longer.

Mesosoma: Precoxal sulcus broader than narrow.

Wings: Fore wing: $\mathrm{r} / 3-\mathrm{SR}=0.3 ; \mathrm{C}+\mathrm{SC}+\mathrm{R} / \mathrm{SR} 1=1.6 ; \mathrm{m}-\mathrm{cu}$ antefurcal; pterostigma yellow-brown. Hind wing: $1-\mathrm{M} / \mathrm{cu}-\mathrm{a}=1.1$

Legs: Hind coxa granulate; hind femur 6.2 times longer than wide; tarsal claws small with lobe.

Metasoma: Petiolar tergum 2.25 times longer than wide apically, 4.5 times fore wing; dorsope absent; ventral folds of petiolar tergum not joined, a little gap remaining; ovipositor straight; length of ovipositor 1.8 times petiolar tergum, and 0.45 times fore wing; propodeum convex, reticulate-rugose and with a central longitudinal carina; Colour: Frons black; occiput and vertex brown; otherwise yellow-brown; pronotum and mesopleuron red-brown; propodeum black with a red-brown patch laterally; petiolar tergum black; 3rd abdominal tergite yellow rest is brown; legs dark yellow to brown.

Distribution: $\underline{\text { Sweden. }}$ 
Biology: We found 5 specimens within the SMTP. Three specimens were caught in alpine birch forest, one was caught in spruce/larch forest, and one was caught in a recycling facility in a spruce forest. The specimens were caught from the end of June to the middle of September.

Etymology: The name stenomastax is derived from the Greek words steno (narrow), and mastax (mouth), referring to the narrow clypeus of this species.

\section{Meteorus subjaculator Tobias}

Fig. 127

Meteorus subjaculator Tobias, 1986:344 Holotype , , Russia: ‘Altai river Katun, 9.viii.1909’ (Gorchakovskiy) (ZIN, St. Petersburg)-examined.

Diagnosis: Meteorus subjaculator is similar to M. cis but has a longer ovipositor (3.5 times the length of the petiolar tergum in the former, 2.0 in the latter). Another character separating these two species is the sculpture of the propodeum. M. subjaculator has the propodeum set with vague carinae, hidden in rugosity, whilst M. cis has distinct carinae and smooth areas in between.

Studied material: 2 specimens.

Description: Size $3.5 \mathrm{~mm}$. Antennal articles 23 . Head slightly transverse but rounded behind eyes. Ocelli small, $\mathrm{OOL}=4$. Eyes small, slightly protuberant. Malar space slightly shorter than basal breadth of mandible. Face 1.2 times as broad as high, punctate, not strongly protuberant. Clypeus narrower than face, almost flat, its apical border weakly notched medially and usually with a weak vertical impression behind the notch. Tentorial pits distinct. Mandibles short, stout and moderately twisted. Precoxal sulcus wide, rugose. Propodeum with slight carinae, quite rugose. Petiolar tergum with small dorsal pits, dorsal surface, from base to apical end, reticulate-rugose with a few longitudinal striae laterally. Ovipositor 3.5 times the length of petiolar tergum, slender. Legs slender; hind coxa punctate. Tarsal claws not lobed but somewhat swollen basally. Colour testaceous to brown; clypeus, mandibles and usually $3^{\text {rd }}$ abdominal tergum reddish testaceous; coxa and legs yellow. Pterostigma dark with light upper border. Fore wing $\mathrm{m}-\mathrm{cu}$ antefurcal, vein 2-SR+M as long as vein $\mathrm{r}$.

Distribution: Russia.

Biology: Unknown.

\section{Meteorus subtilisulcus Stigenberg sp nov.}

Fig. $69,79 \mathrm{~g}, 128$

Diagnosis: The short (1.6 times petiolar tergum), straight ovipositor in combination with the wide and flat clypeus and short malar space ( 0.1 times the width of mandible base) clearly distinguish this species. It mostly resembles $M$. densipilosus, but this species has a longer ovipositor (2.0 times the length of the petiolar tergum), a slightly convex and narrow clypeus and a wider malar space ( 0.5 times the width of mandible base).

Studied material: Holotype † FIN. Turunja Porin Lääni, SSW. Dragsfjärd, 29.vii.1992. M. Söderlund, RMNH'97.

Description: Body $3.5 \mathrm{~mm}$, fore wing $3.8 \mathrm{~mm}$.

Head: Length of eye 0.6 times length of temple in dorsal aspect; eyes large, converging and slightly protuberant; width of face 1.7 times its height; $\mathrm{OOL}=2.3$; vertex smooth but setate; face punctulate; clypeus as wide as face and short in length, slightly longer than mandible width, clypeus distinctly divided, punctate, flat with a sinuate apical border; length of malar space 0.1 times basal width of mandible; mandibles not twisted; antennal articles 27; 3rd antennal article 3.3 times longer than wide; 4th antennal article 3 times longer than wide; all articles longer than wide.

Mesosoma: Precoxal sulcus narrow and shallow.

Wings: Fore wing: $\mathrm{r} / 3-\mathrm{SR}=0.5 ; \mathrm{C}+\mathrm{SC}+\mathrm{R} / \mathrm{SR} 1=1.5 ; \mathrm{m}-\mathrm{cu}$ shortly antefurcal; pterostigma with dark brown center and light borders, the apical corner light. Hind wing: $1-\mathrm{M} / \mathrm{cu}-\mathrm{a}=0.8$;

Legs: Hind coxa smooth to punctate; hind femur 4.1 times longer than wide; tarsal claws slender with lobe. 
Metasoma: Petiolar tergum 1.5 times longer than wide apically; dorsope distinct; ventral folds of petiolar tergum not joined; petiolar tergum striate with dorsal central rugosity running from the base of the tergum past the spiracles and ending surrounded by enclosing striae; ovipositor straight, length of ovipositor sheaths 1.6 times petiolar tergum, and 0.35 times fore wing; propodeum with strong carina, smooth inbetween.

Colour: Dark brown-black: clypeus brown-yellow, legs yellow.

Distribution: Finland.

Biology: The holotype was caught at the end of July.

Etymology: The name subtilisulcus refers to the very subtle impression of the precoxal sulcus.

\section{Meteorus sulcatus Szépligeti}

Fig. 78, 129

Meteorus sulcatus Szépligeti, 1896:310. Holotype , Yugoslavia: Croatia, Grehovica, 10.v.1885 (published as 1882) (Biró) (HNHM, Budapest).

Meteorus insignis Muesebeck, 1939:83. Holotype + , Great Britain: England, Herefordshire, Brickett Wood, 17.vi.1936 (Benson) (BMNH, London) synonymized by Fischer, 1966:395 - examined.

Meteorus molorchi Fischer, 1966:391. Holotype ${ }^{+}$, Austria: 'Österreich, Wien XIII, ob. Tirolergarten, ex Molorchus umbellatarum, vi.1964 (Holzschuh)' (NHM, Vienna) synonymized by Huddleston, 1980:49.

Diagnosis: Meteorus sulcatus is closely related to M. corax and M. nixoni. All are large and dark species with a wide face. However, M. sulcatus is the smallest amongst these with a length of about $4 \mathrm{~mm}, M$. corax and M. nixoni both are about $10 \mathrm{~mm}$. The petiolar tergum of the former is almost parallel whilst on both the latter species it is distinctly widening apically.

Studied material: $\sim 5$ specimens.

Description: Size about $4 \mathrm{~mm}$. Antennae 31-36 articles. Clypeus flat, punctuate. Mandibles stout and twisted. $\mathrm{OOL}=1.5-2.0$. Propodeum depressed, rugose. Petiolar tergum broad, reticulate dorsally. Petiolar tergum longitudinally striate, with distinct thyridia. Ovipositor long, 3.0-3.5 times petiolar tergum. Tarsal claws without basal lobe but swollen at the base. Second submarginal cell much higher than wide.

Distribution: Western and Eastern Palearctic. Country records: Austria; Croatia; Czechoslovakia; France; Germany; Hungary; Japan; Netherlands; Russia; Slovakia; United Kingdom.

Biology: Meteorus sulcatus is recorded as a parasitoid of Coleoptera: Buprestidae, Cerambycidae, and Chrysomelidae (Yu et al. 2005).

\section{Meteorus tabidus (Wesmael)}

Fig. 130

Perilitus tabidus Wesmael, 1835:32. Lectotype ${ }^{\widehat{N}}$, Belgium: Brussels, coll. Wesmael (IRSNB, Brussels).

Meteorus fascialis Ruthe, 1862:22. Holotype $\odot$, Germany. Ruthe coll. (BMNH, London), synonymized by Schmiedeknecht, 1897:187-examined.

Meteorus dubius Ruthe, 1862:27. Holotype $\odot$, Germany. Ruthe coll. (BMNH, London), synonymized by Huddleston, 1980:50 examined.

Meteorus pentheri Fischer, 1970b:262. Holotype đ̂, Yugoslavia: Stolac (Penther) (NHM, Vienna), synonymized by Huddleston, 1980:50.

Diagnosis: Meteorus tabidus is very similar to M. affinis but $M$. tabidus has a more protuberant clypeus, the precoxal sulcus is reticulate-rugose and not foveolate as in M. affinis, and M. tabidus is generally a slightly more robust wasp than M. affinis.

Studied material: $\sim 20$ specimens.

Description: Size about 4.5-5mm. Antennae 28-31 articles, thick, the penultimate five or so articles not longer than broad. Head contracted behind eyes, not distinctly rounded, the occiput not distinctly concave. OOL=2. Eyes large, strongly convergent. Malar space short, about half basal breadth of mandible. Face not strongly convex. 
Clypeus strongly protuberant. Tentorial pits wide and deep, very close to orbits. Mandibles at most moderately twisted. Precoxal sulcus broad, reticulate-rugose. Propodeum strongly rugose, sometimes reticulate-rugose, often with a distinct basal transverse carina and less distinct central longitudinal and medial transverse carinae. Petiolar tergum long, narrow, with distinct dorsal pits. Ovipositor long, 2.5-3.0 times length of petiolar tergum, thick. Legs long; hind coxa strongly rugose; tarsal claws with a distinct basal lobe. Colour black; abdominal tergites 3 and 4 and legs testaceous. The face and base of the antennae sometimes lighter. Male same as female except much darker; the antennae longer, 32-35 articles, the ocelli slightly larger; the eyes not strongly convergent; propodeum depressed.

Distribution: Palearctic. Country records: Austria; Belgium; Bosnia Hercegovina; Bulgaria; Croatia; Czechoslovakia; France; Georgia; Germany; Greece; Hungary; Ireland; Italy; Korea; Lithuania; Mongolia; Netherlands; Poland; Romania; Slovakia; Sweden; Switzerland; United Kingdom; Yugoslavia.

Biology: M. tabidus is recorded in the literature as a parasitoid of both Coleoptera (Cerambycidae) (Giraud et al. 1877) and Lepidoptera (Coleophoridae, Geometridae, Psychidae and Tortricidae). We have not been able to verify any host records.

\section{Meteorus tenellus Marshall sp. reinst.}

Fig. 43, 45, 47, 131

Meteorus tenellus Marshall, 1887:125 Lectotype $\odot$ Great Britain (BMNH)-examined.

Meteorus boreus Tobias, 1986:333. Holotype $\circ$, Russia: Kola Peninsula, 'Vitti-guva, Imandra Lake, Arkhangel'sk Province, 3.ix.1929' (Cheburova) (ZIN. St. Petersburg)-examined. Syn. n.

Diagnosis: Meteorus tenellus is very close to M. cinctellus. M. tenellus has a light yellow pterostigma and M. cinctellus has a dark brown pterostigma with whitish edges. The subapical five antennal articles are longer than broad in M. tenellus ( 0.6 times as wide as long), as long as broad in M. cinctellus (0.8-1.0 times as wide as long).

Studied material: 35 specimens.

Description: Size about 4mm. Antennal articles 27-30, all articles longer than broad. Head strongly contracted behind eyes. $\mathrm{OOL}=2$. Eyes protruding and large. Malar space $0.4-0.6$ times basal width of mandibles. Face slightly (0.9-1.1 times) broader than high and with a medial tubercle just below the antennal sockets. Clypeus protuberant and with long setae, mandibles small and strongly twisted. Precoxal sulcus deep and broad, rugose, otherwise area smooth. Propodeum with a longitudinal carina that sometime is hard to distinguish from other rugosity. Petiolar tergum long, slender and without dorsal pits. Ovipositor 2.0-4.0 times petiolar tergum. Fore wing 5 times the length of petiolar tergum. Hind coxa rugose dorsally. Forewing m-cu postfurcal or interstitial. Colour generally dark, face yellow to brown, sometimes with lighter details on the head, the legs yellow to brown.

Distribution: Western Palearctic. Country records: Austria; Finland; Lithuania; Russia: Sweden; United Kingdom; Yugoslavia.

Biology: This species was described by Marshall (1887) from six females bred by Bignell on the host Acleris hastiana (Lepidoptera, Tortricidae ).

Remarks: This species, distributed in Sweden, Lithuania and Russia, was synonymyzed by Huddleston (1981) under M. cinctellus (Spinola 1808), a name that later turned out to be a synonym of M. cinctellus (Fabricius 1777) (Yu et al. 2005). Examination of the types of M.tenellus and M. cinctellus, and of specimens from a variety of locations, has led us to conclude that M.tenellus is a good species.

\section{Meteorus versicolor (Wesmael)}

Fig. 26, 33, 132

Perilitus versicolor Wesmael, 1835:43. Lectotype $\odot$, Belgium: Charleroy (IRSNB, Brussels), designated by Marsh (1979).

Perilitus bimaculatus Wesmael, 1835:45. Lectotype $q$, Belgium: Charleroy (IRSNB, Brussels), designated by Marsh (1979), synonymized by Muesebeck, 1923:36.

Perilitus unicolor Hartig, 1838:245. Lectotype + , Germany (ZSBS, Münich), (Junior primary homonym of unicolor Wesmael 1835). 
Perilitus brevicornis ratzeburg, 1844:77. Holotype , Germany: Ratzeburg coll (IP, Eberswalde), synonymized by Köningsmann, 1964:654.

Perilitus rugator Ratzeburg, 1852:59. Lectotype $~$, Germany:'741/neust Br./Peril rugator and 30 art', Ratzeburg coll. (IP, Eberswalde), synonymized by Huddleston, 1980:52.

Meteorus decoloratus Ruthe, 1862:48. Lectotype ${ }_{+}$, Germany: Ruthe coll. (BMNH, London), synonymized by Muesebeck, 1923:36-examined.

Meteorus camptolomae Watanabe, 1939:25. Holotype $q$, Japan: ‘Okikuba, Tokyo’ (EI, Sapporo), synonymized by Huddleston, 1980:52.

Meteorus ikonomovi Fischer, 1959:5. Holotype , Yugoslavia: Dalmatien, Gravosa (NHM, Vienna), synonymized by Huddleston, 1980:52.

Meteorus hartigi Shenefelt, 1969:69 (Replacement name for unicolor Hartig, 1838), synonymized by Huddleston, $1980: 52$.

Diagnosis: This species is most similar to $M$. obsoletus. The compound eye of $M$. versicolor is more protruding and the ovipositor is thicker and slightly shorter than that of M. obsoletus. Also, the temples are shorter and more flat in M. versicolor, appearing rather narrow in dorsal view. In M. obsoletus, the temples are longer and more convex, appearing more rounded and less narrow in dorsal view.

Studied material: $\sim 100$ specimens.

Description: Size about 4.5-5mm. Antennae with 29-33 articles; flagellum thick basally, tapering to apex, all articles of flagellum distinctly longer than broad. Head broad, more or less strongly contracted behind eyes. Ocelli large, $\mathrm{OOL}=\mathrm{OD}$, protuberant. Eyes large, protuberant, only slightly convergent. Malar space short, always less than the basal breadth of the mandible. Face about 1,5 times as broad as high, not strongly protuberant but somewhat raised medially. Clypeus protuberant. Mandibles small, delicate, strongly twisted. Precoxal sulcus weakly rugose and foveolate, generally broader. Propodeum short, broad, strongly rugose, often reticulate-rugose with no distinct carinae but with a distinct medial impression posteriorly. Petiolar tergum long, rather slender at the base with no dorsal pits, its ventral borders joined from shortly before the midpoint of the segment to its base, dorsal surface finely striate. Ovipositor short, 1.5-2.0 times length of petiolar tergum, thick, strongly tapered shortly before apex. Legs stout, hind coxa smooth, punctate, often with weak transverse rugae dorsally; tarsal claws strongly curved with a pronounced basal lobe. Colour varies from almost completely testaceous to almost completely brown. The base of petiolar tergum generally pale yellow. Male same as female except that eyes generally are smaller and less protuberant. Pale yellow base of petiolar tergum is easily seen but check also the size of ocelli to be sure.

Distribution: Western and Eastern Palearctic. Country records: Armenia; Austria; Azerbaijan; Belgium; Bulgaria; Canada; China; Croatia; Czechoslovakia; Finland; France; Germany; Greece; Hungary; Ireland; Israel; Italy; Japan; Korea; Latvia; Lithuania; Madeira Islands; Moldova; Mongolia; Netherlands; Norway; Palestine; Poland; Portugal Romania; Russia; Slovakia; Spain; $\underline{\text { Sweden; Switzerland; Tajikistan; Turkey; USA; Ukraine; United }}$ Kingdom; Uzbekistan; Yugoslavia.

Biology: Meteorus versicolor is recorded as a parasitoid of 14 different lepidopteran families. There are also some records indicating that the species parasitizes different families of Chalcidoidea or the Ichneumonidae (Yu et al. 2005).

\section{Meteorus vexator (Haliday)}

Fig. 58, 63, 133

Perilitus vexator Haliday, 1935:33. Neotype , Ireland: Co. Kd., R. Canal, (2), 11.vii.1943 (Stelfox) (USNM, Washington), designated by Fischer (1959:12).

Diagnosis: Meteorus vexator is close to M. affinis but M. affinis has lobed claws (simple in M. vecator) and larger ocelli, characters that are distinct also in small specimens that are otherwise quite difficult to separate because of their weaker body sculpture and smaller number of antennal articles. Furthermore, the mesosoma of M. affinis sometimes has yellow markings, unlike $M$. vexator.

Studied material: $\sim 100$ specimens.

Description: Size about 2.5-4mm. Antennae 20-24 articles, slender, all flagellar articles distinctly longer than broad. Ocelli small, $\mathrm{OOL}=2.5-3.0$. Eyes large, protuberant, very strongly convergent. Malar space short, much less than basal breadth of mandible. Face about as broad as high, not protuberant, smooth, punctate. Clypeus moder- 
ately protuberant, smooth, punctate. Mandibles short, stout, moderately twisted. Pronotum laterally generally with weak rugose sculpture. Precoxal sulcus narrow, foveolate, sometimes with a small punctate patch beneath. Propodeum small, distinctly carinate, generally rather smooth between the carinae but sometimes weakly rugulose. Petiolar tergum with distinct dorsal pits. Ovipositor 2.5-3.0 times length of petiolar tergum. Legs long, slender, hind coxa generally at least slightly rugose ventro-laterally though sometimes reticulate-punctate; tarsal claws without a basal lobe. Colour brownish black. Male same as female except antennae longer, up to 27 articles; eyes less convergent and sculpture of precoxal sulcus and of hind coxa sometimes obsolete.

Distribution: Western Palearctic. Country records: Austria; Bulgaria; Croatia; Czechoslovakia; Denmark; Finland; Germany; Hungary; Ireland; Latvia; Netherlands; Russia; Slovenia; Sweden; Switzerland; United Kingdom; Yugoslavia.

Biology: Meteorus vexator has been recorded as a parasitoid of Biphyllidae (Coleoptera) (Morley 1912) but as Huddleston points out, this record is doubtful. We received specimens from T. Munk that were reared from Dahlica lichinella (Lepidoptera: Psychidae), which is more in line with expectations.

Species still classified as nomina dubia, the types lost or not found:

Perilitus (Meteorus) delator (Haliday, 1874). Type material not found.

Perilitus dilatus (Ratzeburg, 1844). Type material lost.

Meteorus effeminatus Ruthe, 1862. These male specimens were examined by Huddleston in 1981 and by JS in 2010 without any conclusion about their placement within Meteorus.

Perilitus flaviceps (Ratzeburg 1844). Type material lost.

Perilitus longicornis (Ratzeburg 1844). Type material lost.

Meteorus stenostigma Thomson 1895. Type material lost. The two specimens in Thomson's collection above the name stenostigma were determined by JS as M. ictericus Nees, 1811, and M. pendulus (Müller, 1776). Neither of them agrees with Thomson's description and neither is from the type locality (Huddleston 1980).

\section{Acknowledgements}

We wish to thank the following people for loan of material and assistance in the study of specimens: S. Belokobylskij (ZIN), R. Danielsson and R. Bygebjerg (MZLU), H. Mejlon (UUZM), G. Broad and D. Notton (BMNH), S. Shaw (UWIM), D. Zimmermann (NHMW), F. Koch (ZMHB), O. Tadauchi (Kyushu University), T. Munk, P. Grootaert (IRSNB), Y. Braet, and A. Ribes Escolá. A. Boring (Univ. of Kentucky) supplied us with outgroup sequences and a lot of helpful advice on euphorines. JS is most grateful to Dr M. Forshage and Dr J. Ferrer for taxonomical help. We are particularly grateful to Prof. C van Achterberg for sharing data and for helping this study along in many important ways, and to Dr J. Jennings, The University of Adeliade for valuable comments on the manuscript. The work was supported by a grant from The Swedish Taxonomy Initiative (Dha 167/08 1.4). 

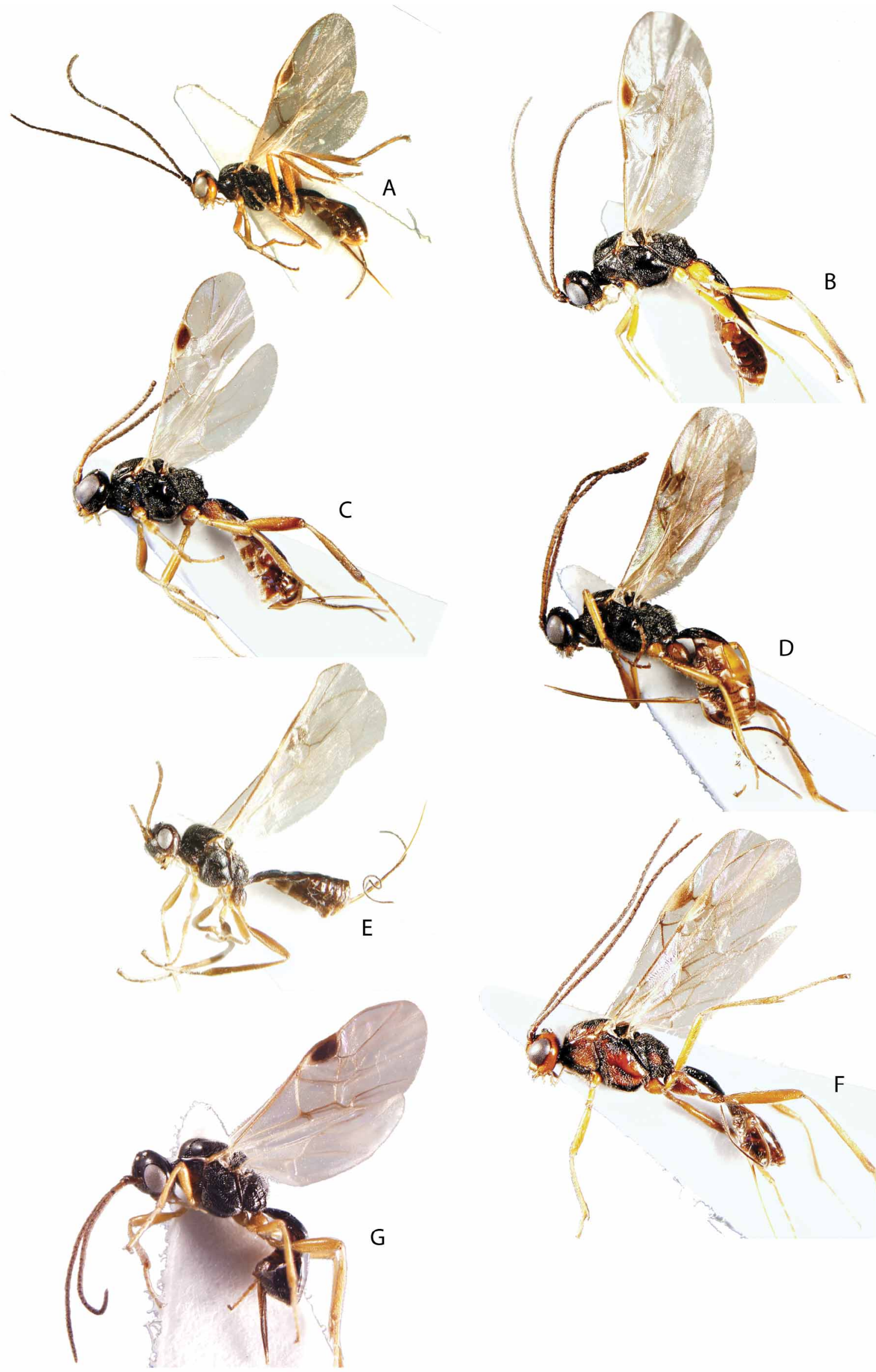

FIGURE 79. Habitus images of the seven new species described in this paper. A) Meteorus artocercus, B) M. densipilosus, C) M. eklundi, D) M. longipilosus, E) M. sibyllae, F) M. stenomastax, G) M. subtilisulcus. 

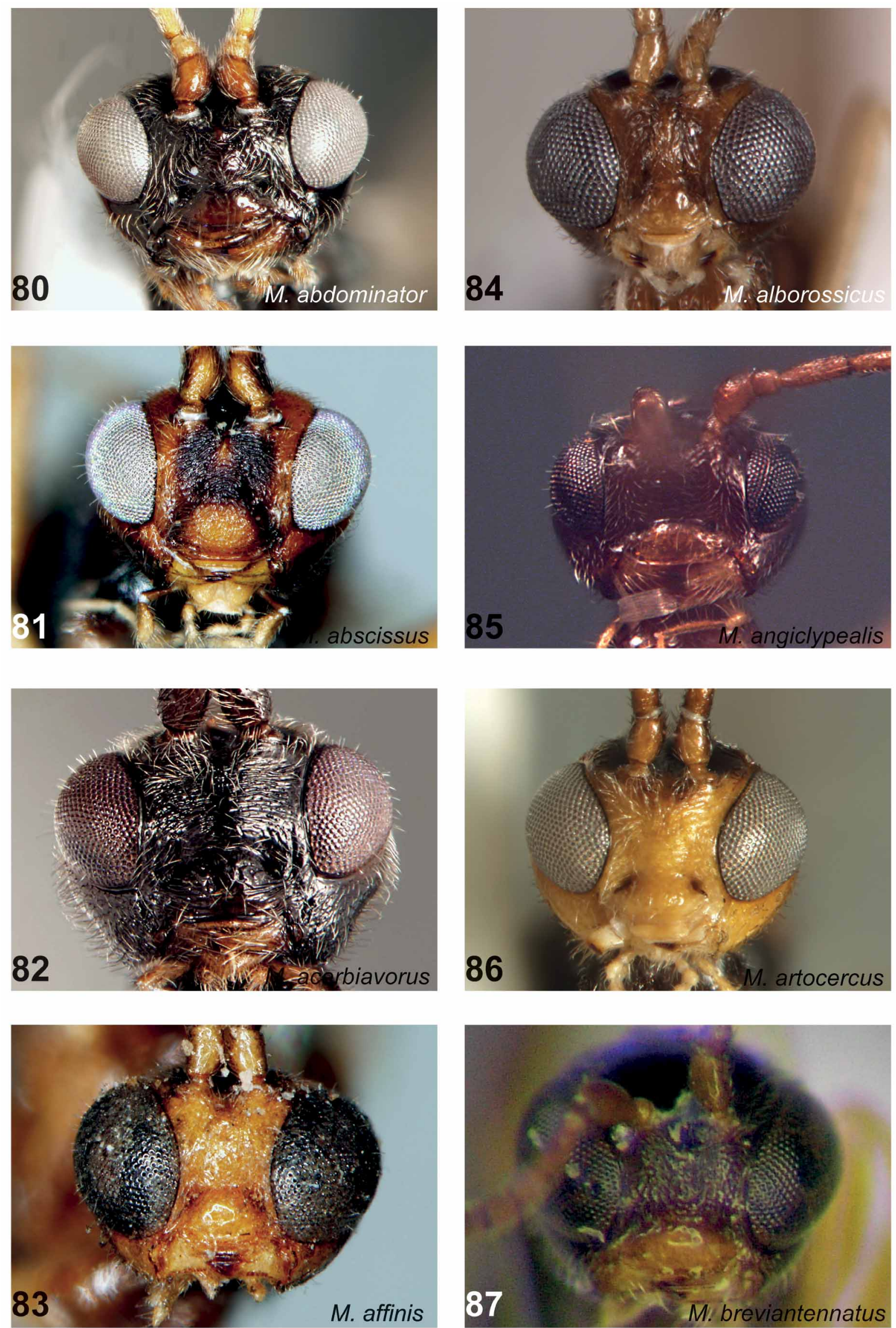

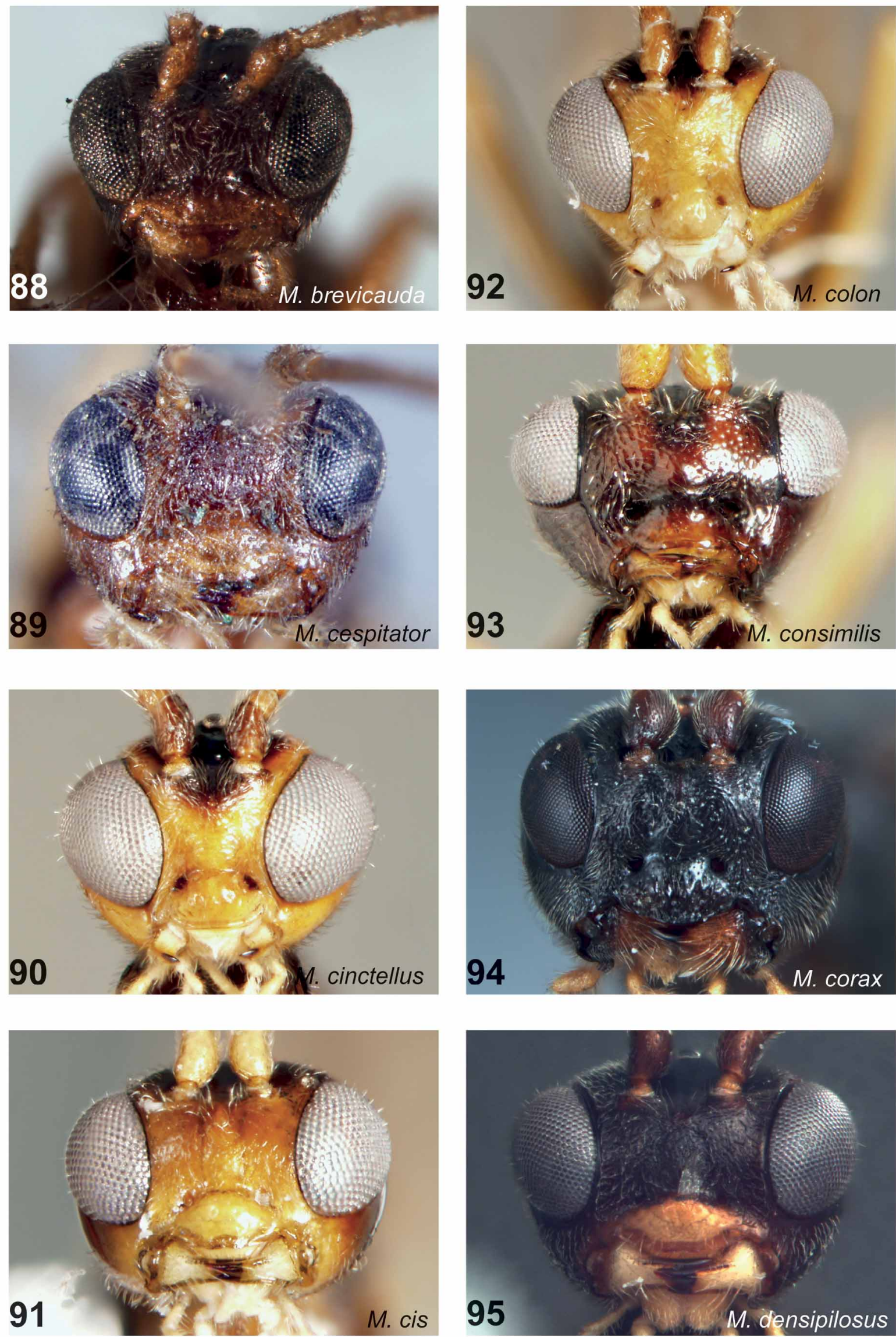

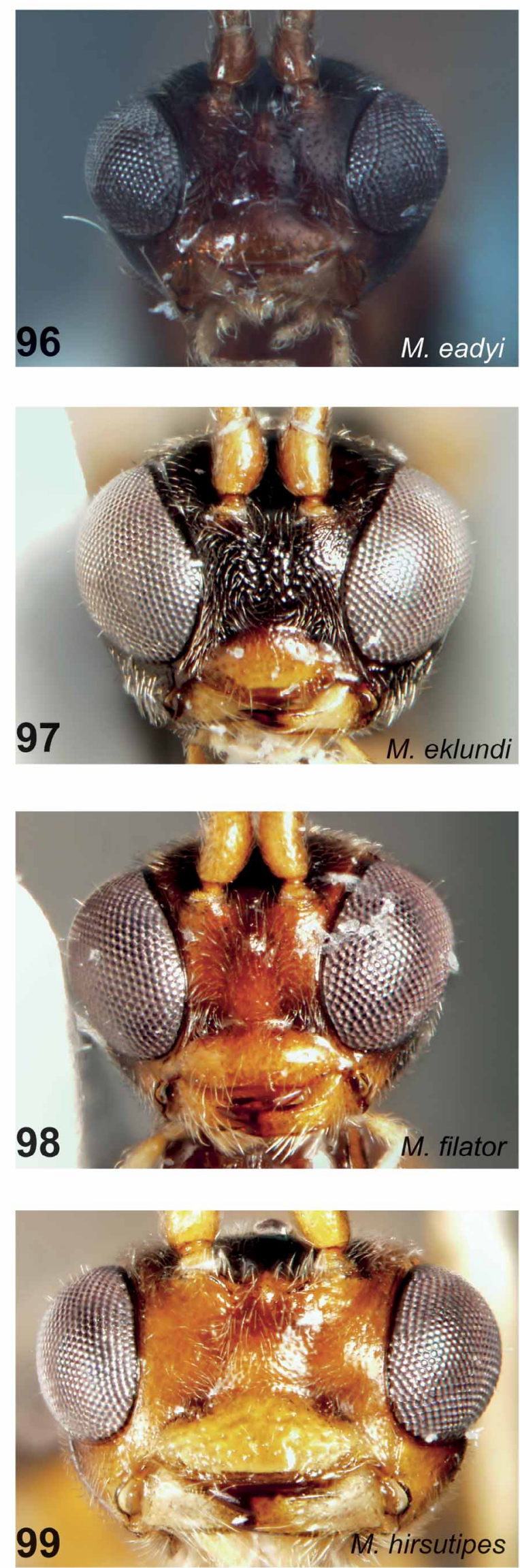
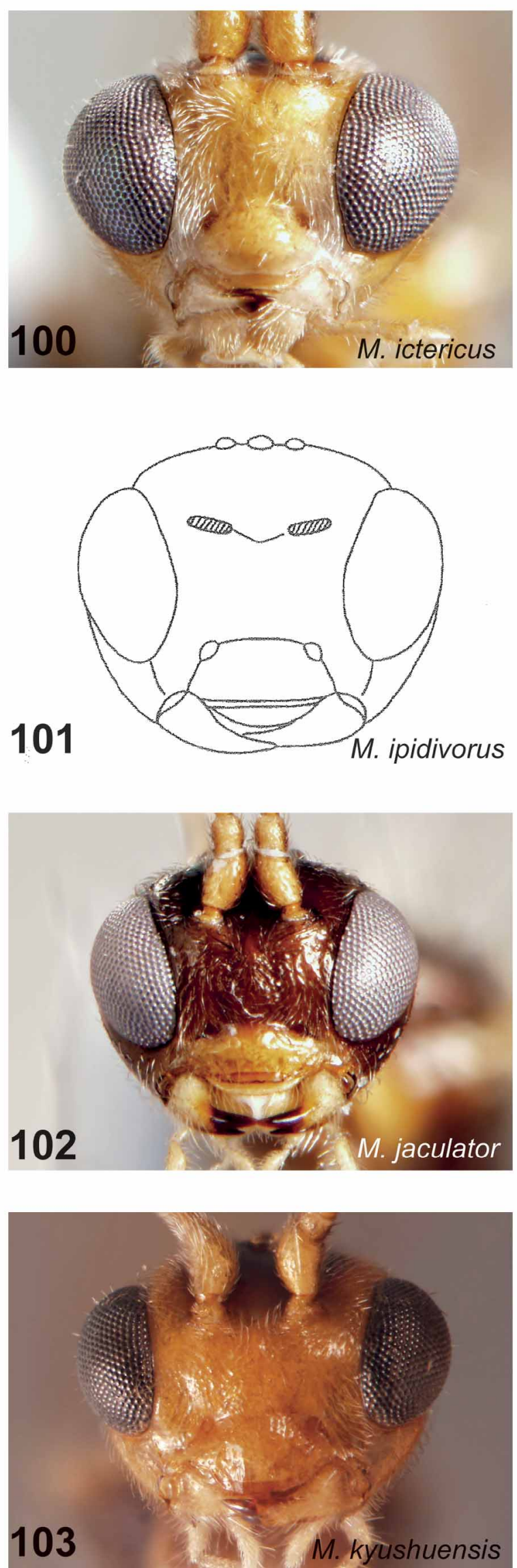

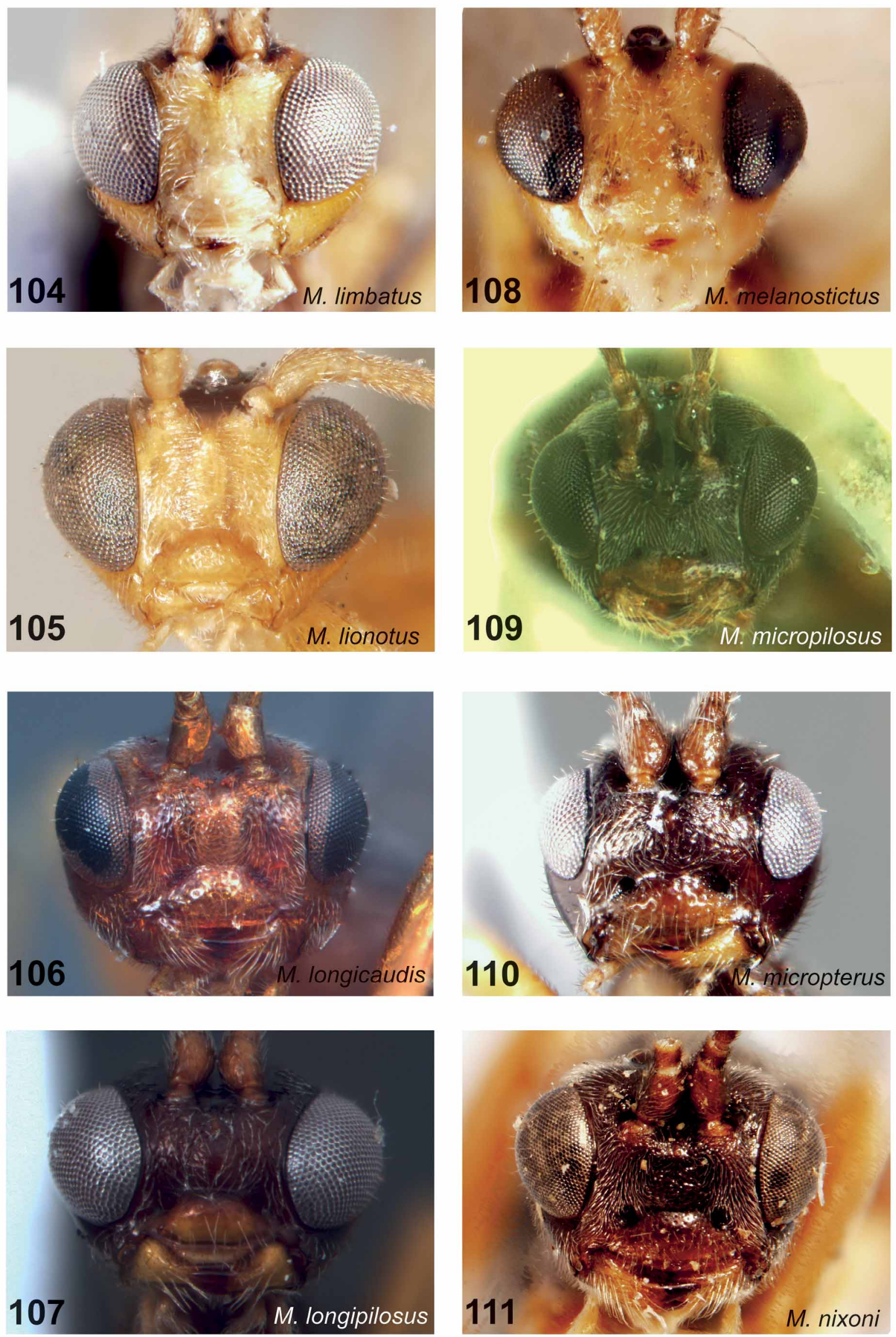

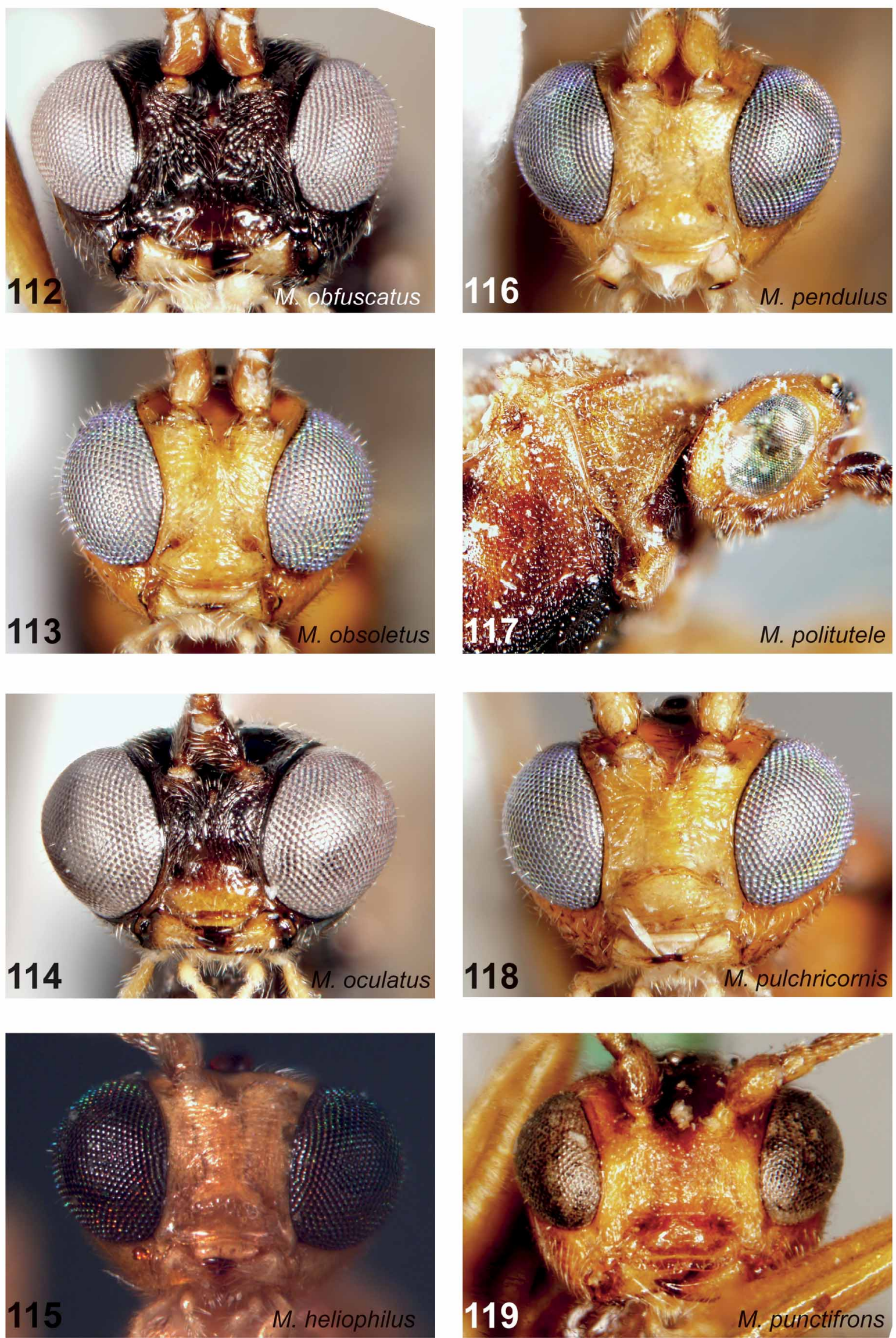

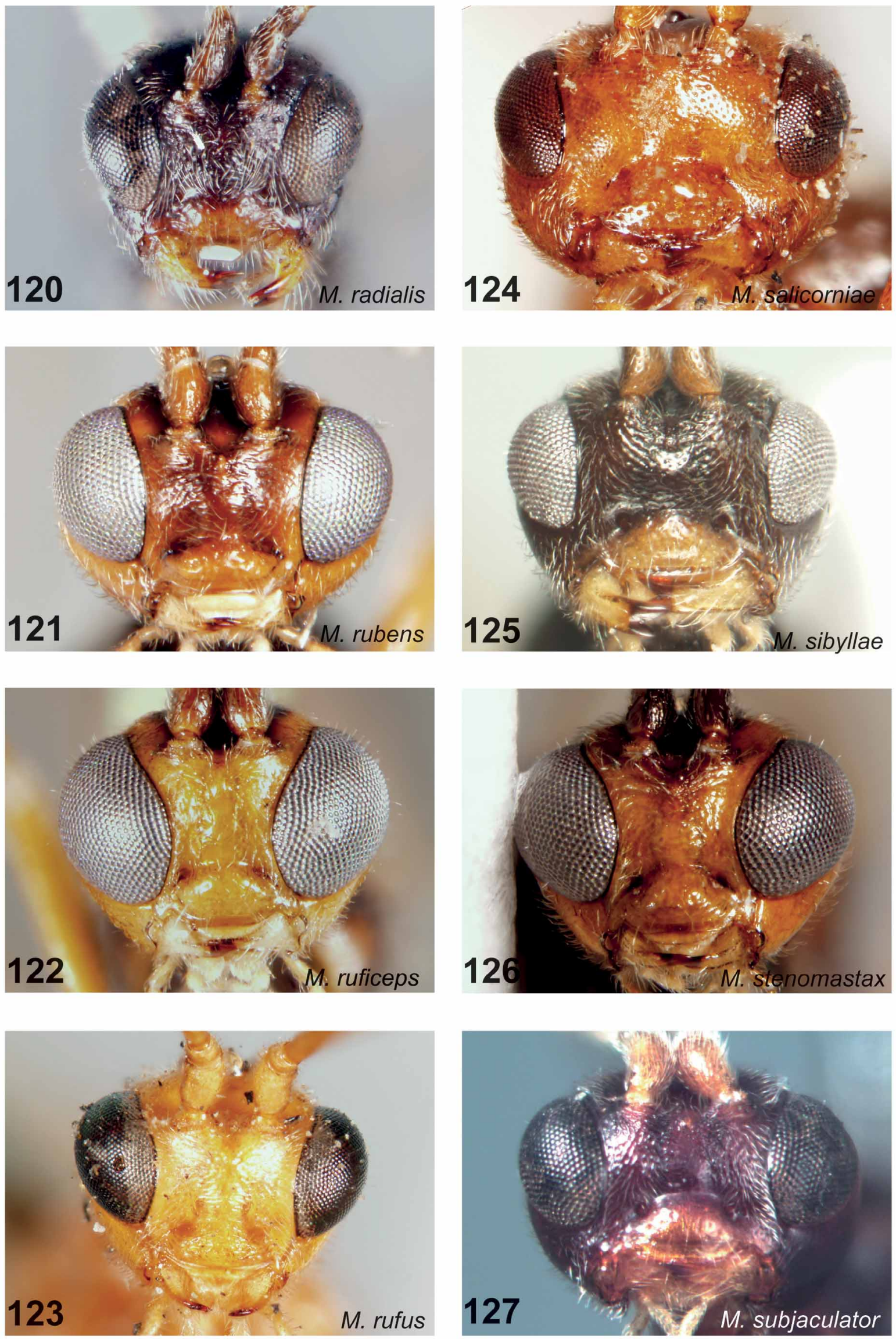

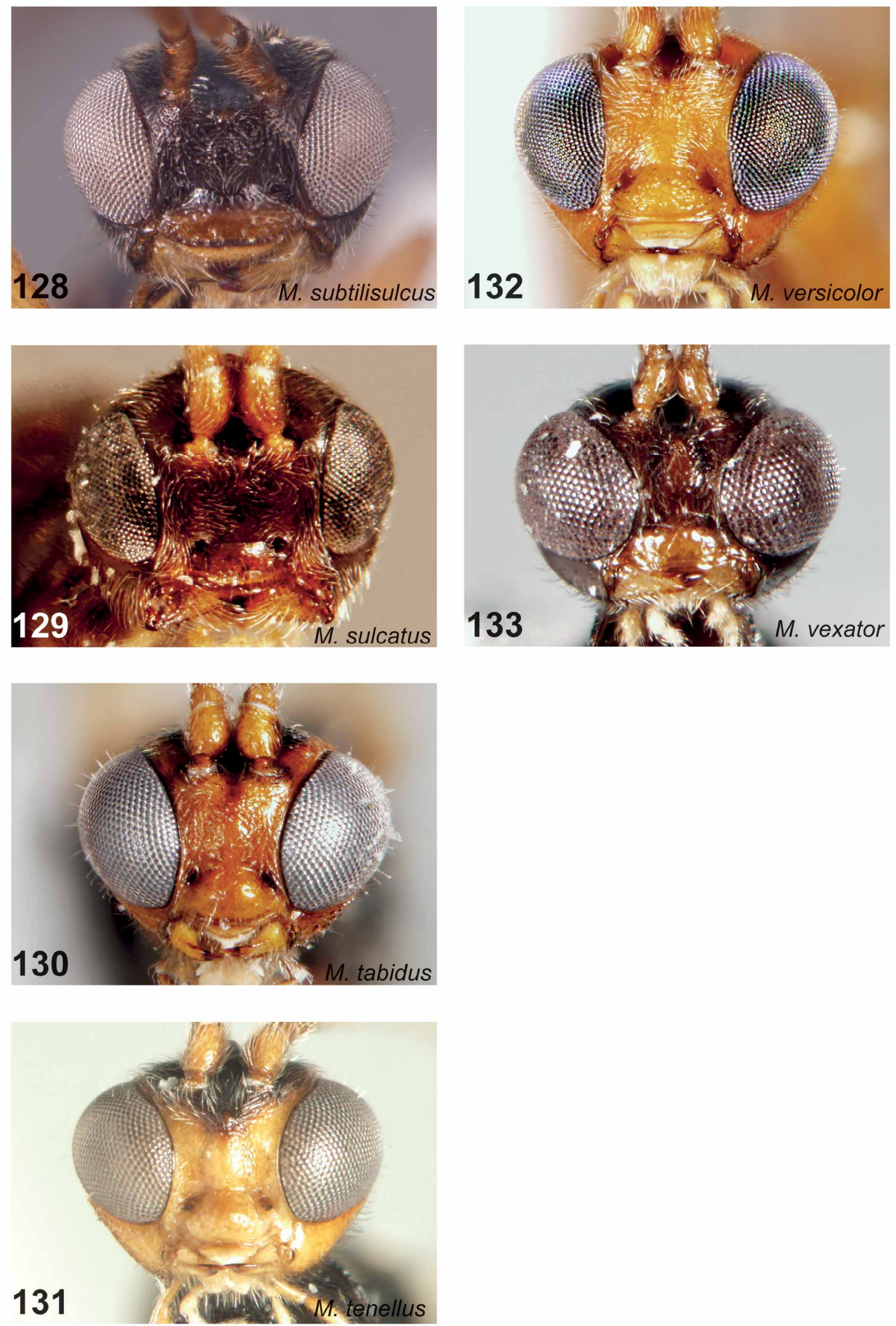

FIGURES 80-133. Anterior view of the head of Meteorus species (except for M. politutele, which is depicted in lateral view). 

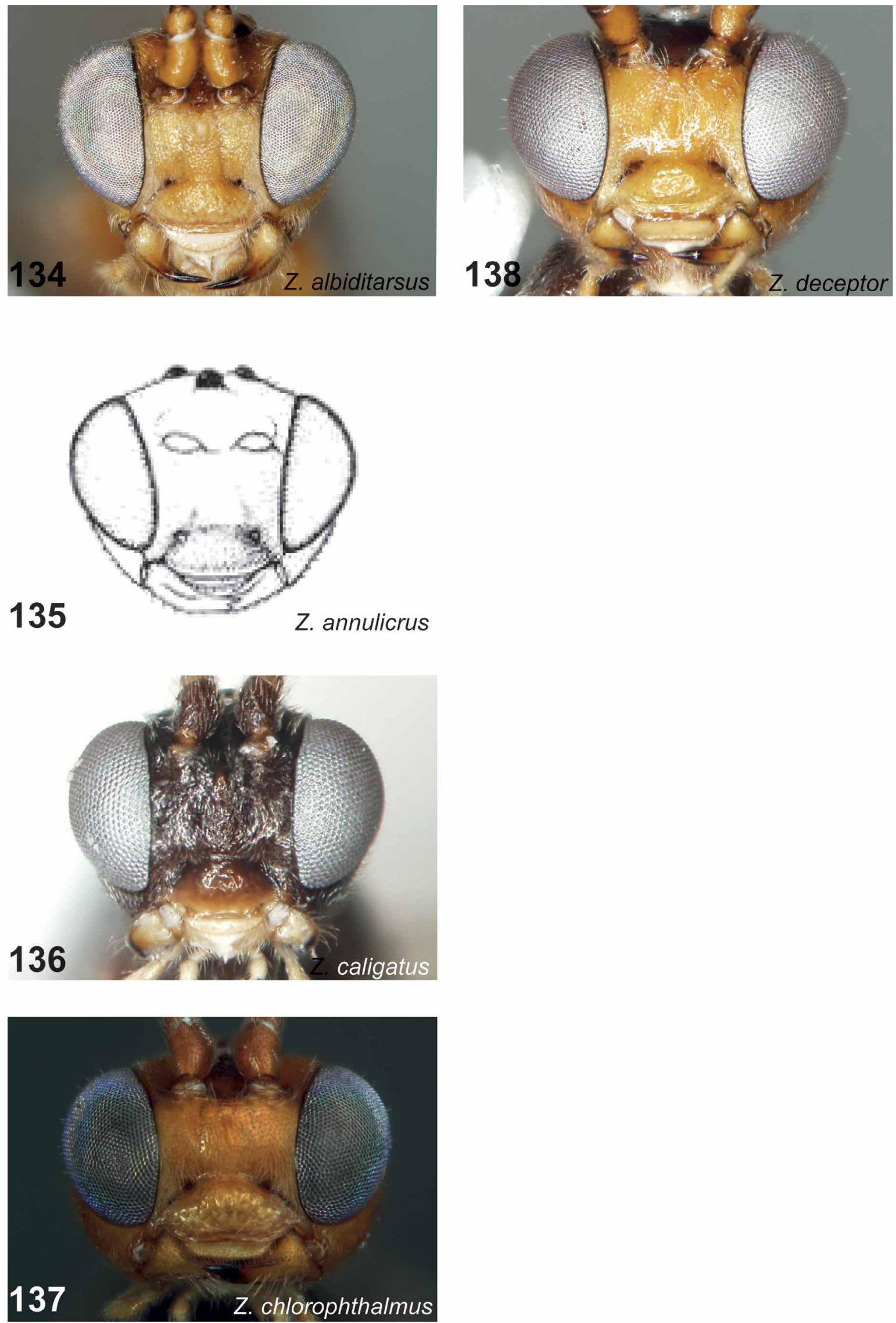

FIGURES 134-138. Anterior view of the head of Zele species. 


\section{References}

Achterberg, C. van. (1979) A revision of the subfamily Zelinae auct. (Hymenoptera, Braconidae). Tijdschrift voor Entomologie, $122,241-479$.

Achterberg, C. van. (1984) Essay on the phylogeny of Braconidae (Hymenoptera: Ichneumonoidea). Entomologisk Tidskrift, $105,41-58$.

Achterberg, C. van. (1984) Addition to the revision of the genus Zele Curtis (Hymenoptera: Braconidae). Entomologishe Berichten, 44, VII, 110-112.

Achterberg, C. van. (1988) Revision of the subfamily Blacinae Foerster (Hymenoptera, Braconidae). Zoologische verhandelingen, 249, 1-324.

Achterberg, C. van. (2001) Two new species of Braconidae (Hymenoptera: Euphorinae, Braconinae) from Switzerland and The Netherlands. Zoologische Mededelingen, 75, 397-403.

Achterberg, C. van. (2004) Fauna Europaea: Hymenoptera, Braconidae. Fauna Europaea version 1.1. Available from: http:// www.faunaeur.org (accessed 29 June 2010)

Achterberg, C. van \& Franquinho Aguiar, A.M. (2009) Additions to the fauna of Braconidae from Madeira and Selvagens Islands, with the description of five new species (Hymenoptera: Braconidae: Homolobinae, Alysiinae, Opiinae). Zoologische Mededelingen, 83, 777-797

Aguirre F., H., Shaw, S.R. \& Jones, G.Z. (2010) A new Meteorus Haliday species from Colombia and Ecuador (Hymenoptera: Braconidae). Zootaxa, 2453, 55-61

Aguirre, H., Sarmiento, C.E., Shaw, S.R. (2011) Taxonomic revision and morphometric analyses of Meteorus Haliday, 1835 (Hymenoptera: Braconidae: Meteorinae) from Colombia. Zootaxa, 2938, 1-68.

Ashmead, W.H. (1906) Description of new Hymenoptera from Japan. Proceedings of the United States National Museum, 30 , 169-201.

Askari, A., Mertins, J.W. \& Coppel, H.C. (1977) Developmental biology and immature stages of Meteorus pulchricornis in the laboratory. Annals of the Entomological Society of America, 70, 655-659.

Askari, A. \& Coppel, H.C. (1977) Adult biology and effect of different food sources on longevity and fecundity of the gypsy moth parasitoid, Meteorus pulchricornis (Wesmael) (Hymenoptera: Braconidae). University of Wisconsin Forestry Research Notes, 207, 6 pp.

Askari, A. \& Coppel, H.C. (1978) Observations on courtship and mating behavior of Meteorus pulchricornis, a gypsy moth parasitoid. Annals of the Entomological Society of America, 71, 364-366.

Belokobylskij, S. A., (2000) Subfamily Euphorinae. In Lehr P. A. (ed.): Key to Insects of the Russian Far East. Neoropteroidea, Mecoptera, Hymenoptera. Vladivostok, Dal'nauka, 4, 8-26.

Belokobylskij, S.A., Taeger, A., Achterberg, C. van., Haeselbarth, E., Riedel, M. (2003) Checklist of Braconidae of Germany (Hymenoptera), Beiträge zur Entomologie, 53, 341-435.

Belshaw, R., Dowton, M., Quicke, D.L.J. \& Austin, A.D. (2000) Estimating ancestral geographical distributions: A gondwanan origin for aphid parasitoids? Proceedings of the Royal Society of London B, 267, 491-496.

Belshaw, R. \& Quicke, D.L.J. (2002) Robustness in Ancestral State Estimates: Evolution of Life History Strategy in Ichneumonid Parasitoids. Systematic Biology, 51, 450-477.

Berry, J.A. \& Walker, G.P. (2004) Meteorus pulchricornis (Wesmael) (Hymenoptera: Braconidae: Euphorinae): An exotic polyphagous parasitoid in New Zealand. New Zealand Journal of Zoology, 31, 33-44.

Boie, F. (1850) Entomologische Beiträge. Stettiner Entomologische Zeitung, 11, 212-216.

Boring, C.A. (2010) Morphology and systematics of braconid wasps. PhD. Dissertation University of Kentucky. 123pp.

Bouché, P.F. (1834) Naturgeschichte der Insekten, besonders in Hinsicht ihrer ersten Zustande als Larven und Puppen. Lieferung 1. Berlin, Nicolai, 8vo. v, 216 pp.

Broad, G.R., Shaw, M.R. \& Godfray, H.C.J. (2009) Checklist of British and Irish Braconidae (Hymenoptera). [online version at http://www.nhm.ac.uk/resources-rx/files/braconidae-checklist-final-34139.pdf ]

Buffington, M.L., Nylander, J.A.A. \& Heraty, J.M. (2007) The phylogeny and evolution of Figitidae (Hymenoptera: Cynipoidea). Cladistics, 23, 403-431.

Cameron, P. (1912) Description of New genera and Species of Parasitic Hymneoptera taken at Kuching, Sarawak, Borneo by Mr. John Hewitt B.A. Societas entomologica, 14, 84-85

Campbell, B.C. Steffen-Campbell, J.D. \& Werren, J.H. (1993) Phylogeny of the Nasopnia species complex (Hymenoptera: Pteromalidae) inferred from internal transcribed spacer (ITS2) and 28S rDNA sequences. Insect Molecular Biology, 2, $225-237$.

Chau, N.N.B. \& Maeto, K. (2009) Temporary host paralysis and avoidance of self-superparasitism in the solitary endoparasitoid Meteorus pulchricornis. Entomologia Experimentalis et Applicata, 132, 250-255

Chen, X. \& Achterberg, C. van. (1997) Revision of the subfamily Euphorinae (excluding the tribe Meteorini Cresson) (Hymenoptera: Braconidae) from China. Zoologische verhandelingen, 313, 104-116.

Chen, J. \& Wu, Z. (2000) Systematics Studies on Meteorinae of China (Hymenoptera: Braconidae). Huayu Nature Book Trading Co. Ltd., Beijing, 230 pp.

Chau, N.N.B. \& Maeto, K. (2009) Temporary host paralysis and avoidance of self-superparasitism in the solitary endoparasitoid Meteorus pulchrichornis. Entomologia Experimentalis et Applicata, 132, 250-255. 
Clausen, C.P. (1940) Entomophagous Insects. McGraw-Hill Book Company, Inc. New York \& London.

Costa, A. (1884). Notizie ed osservationi sulla geofauna Sarda. Napoli: Reale Academia delle Scienze Fisiche Matamatiche, $22,171-172$.

Curtis, J. (1832) British entomology. 9, folio 415, London.

Dalla Torre, C.G. (1898) Catalogus Hymenopterorum hucusque descriptorum systematicus et synonymicus 4. Guilelmi Engelmann Lipsiae, pp 350.

Dowton, M., Austin, A.D. \& Antolin, M.F. (1998) Evolutionary relationships among the Braconidae (Hymenoptera: Ichneumonidea) inferred from partial 16S rDNA gene sequences. Insect Molecular Biology, 7, 129-150.

Dowton, M., Belshaw, R., Austin, A. \& Quicke, D.L.J. (2002) Simultaneous molecular and morphological analysis of braconid relationships (Insecta: Hymenoptera: Braconidae) indicates independent mt-tRNA gene inversions within a single wasp family. Journal of Molecular evolution, 54, 210-226.

Fahringer, J. (1928) Opuscula Braconologica. Band1, Palaearktischen Region, 433-606pp.

Fahringer, J. (1935) Swedisch-chinesische wissenschaftliche Expedition nach den nordwestlichen Provinzen Chinas, 26 Hymenoptera, 4 Braconidae Kirby. Arkiv för Zoologi, 27(A) (12), 1-15.

Farris, J.S., Albert, V.A., Kjällersjö, M., Lipscomb, D. \& Kluge, A.G. (1996) Parsimony jackknifing outperforms neighbor-joining. Cladistics, 12, 99-124

Fernández-Triana, J.L. (2010) Eight new species and an annotated checklist of Microgastrinae (Hymenoptera, Braconidae) from Canada and Alaska. ZooKeys, 63, 1-53.

Fischer, M. (1957a) Neue Paläarktische Meteorus-Arten (Hymenoptera, Braconiddae) Annalen des Naturhistorischen Museums in Wien, 61, 104-109.

Fischer, M. (1957b) Zur Kenntnis der Gattung Meteorus Haliday (Hymenoptera, Braconidae) Opuscula Zoologica, 3, 1-5.

Fischer, M. (1957c) Beträge zur Kenntnis der Paläarktischen Braconiden. Mitteilungen der Münchner entomologischen Gesellschaft, 47, 1-21.

Fischer, M. (1959a) Zur Kenntnis der Thomson'schen Braconiden-Arten V (Hymenoptera). Entomologisches Nachrichtenblatt Österreichischer und Schweizer Entomologen, 9, 10-11.

Fischer, M. (1959b) Neue und wenig bekannte Braconiden aus Jugoslavien (Hymenoptera). Acta Musei Macedonici scientiarum naturlium, 6.

Fischer, M. (1966) Gezuchtete Braconiden aus Niederösterreich und aus dem Burgenland (Hymenoptera). Zeitschrift für angewandte Entomologie, 53, 385-402.

Fischer, M. (1970b) Die Meteorus-Arten des Burgenlandes (Hymenoptera, Braconidae, Euphorinae). Wissenschaftliche Arbeiten aus dem Burgenland, 44.

Foerster, (1862) Synopsis der Familien und gattungen der Braconen. Verhandlungen des naturhistorischen Vereines der preussischen Rheinlande und Westphalens, 19, 225-228.

Folmer, O., Black, M., Hoeh, W., Lutz, R. \& Vrijenhoek, R. (1994) DNA primers for amplification of mitochondrial cytochrome c oxidase subunit I from diverse metazoan invertebrates. Molecular and Marine Biology and Biotechnology, 3, 294-297.

Fuester, R.W., Taylor, P.B., Peng, H. \& Swan, K. (1993) Laboratory biology of a uniparental strain of Meteorus pulchricornis (Hymenoptera: Braconidae), an exotic larval parasite of the gypsy moth (Lepidoptera: Lymantriidae). Annals of the Entomological Society of America, 86, 298-304.

Giraud, J.É. \& Laboulbène, A. (1877) Liste des eclosions d'insectes observees par le Dr. Joseph-Étienne Giraud, membre honoraire. Annales de la Société Entomologique de France, 5, 397-436.

Goloboff, P.A., Farris, J.S., Källersjö, M., Oxelman. B., Ramírez, M.J. \& Szumik, C.A. (2003) Improvements to resampling measures of group support. Cladistics, 19, 324-332.

Goloboff, P., Farris, J.S., Nixon, K.C. (2008) TNT software version 1.1. A free program for phylogenetic analysis. Cladistics, 24, 774-786.

Haliday, A.H. (1835) Essay on parasitic Hymenoptera. The Entomological magazine, 3, $20-45$.

Hall, T.A. (1999) BioEdit: a user-friendly biological sequence alignment editor and analysis program for Windows 95/98/NT. Nucleic Acids Symposium Series, 41, 95-98.

Hammond, H.E. \& Smith, K.G.V. (1957) On some parasitic Diptera and Hymenoptera bred from Lepidopterous hosts; Part III. Records of Tachinidae (Dip.), Braconidae, Ichneumonidae, Pteromalidae, Eulophidae and Scelionidae (Hym.). Entomologist's Gazette, 8, 181-189

Hartig, T. (1838) Ueber den Raupenfrass im Köngl. Charlottenberger Forste unfern Berlin, wärend des Sommers 1837. Jahresberichte über die Fortschritte der Forstwissenschaft und Forstlichen Naturkundede im Jahre 1836 und 1837 nebst Original-Abhandlungen aus dem Gebiete und Cameralisten, 1, 246-274

Hedqvist, K-J. (1998) Bark beetle enemies in Sweden II. Braconidae (Hymenoptera). Entomologica scandinavica, Supplement No 52.

Hinton, H.E. (1956) The larvae of the species of Tineidae of economic importance. Bulletin of Entomological Research, 47, 251-346.

Holmgren, A.E. (1868) Kongliga fregatten Eugenies resa omkring Jorden. Vetenskapliga iaktagelser. Zoologi; 1 Insecta, häfte 12 hymenoptera, 391-442 pp.

Huddleston, T. (1980) A revision of the Western Palaearctic species of the genus Meteorus (Hymenoptera: Braconidae). Bul- 
letin of the British Museum (Natural History), Entomology series, 41, 1-58.

Huddleston, T. (1983) Meteorus (Hymenoptera: Braconidae) of Australia and New Guinea. Systematic Entomology, 8, 393420.

Huddleston, T. \& Gauld, I. (1988) Parasitic wasps (Ichneumonidae) in British light-traps. Entomologist, 107, 134-154.

Huelsenbeck, J.P. \& Ronquist, F. (2001) MRBAYES: Bayesian inference of phylogenetic trees. Bioinformatics, 17, 754-755.

International Commission of Zoological Nomenclature [ICZN] (1999) International code of zoological nomenclature [the Code]. Fourth edition. The International Trust for Zoological Nomenclature, c/o Natural History Museum, London, i-xxix, + 306 pp. [online version at http://www.iczn.org/iczn/index.jsp]

Li, F.F., Chen, X.X., Piao, M.H., He, J.H. \& Ma, Y. (2003) Phylogenetic relationships of the Euphorinae (Hymenoptera: Braconidae) based on the D2 variable region of 28S ribosomal RNA. Entomotaxonomia, 25, 217-225.

Loan, C.C. (1974). Hosts and generic relations in the euphorini (Hymenoptera, Braconidae). Contributions of the American Entomological Institute, 20, 388-397

Karlsson, D., Papae, T., Johanson, K.A., Liljeblad, J. \& Ronquist, F. (2005) Svenska Malaisefälleprojektet, eller hur många arter steklar, flugor och myggor finns i Sverige? Entomologisk Tidskrift, 126, 43-53.

Katoh, K., Asimenos, G. \& Toh, H. (2009) Multiple Alignment of DNA Sequences with MAFFT. In Bioinformatics for DNA Sequence Analysis edited by D. Posada. Methods in Molecular Biology, 537, 39-64.

Kornoşor, S. (2000). Entomological problems of maize in Turkey. Proceedings of the XXth International Working Group on Ostrinia and other maize pests, 14-23.

Lyle, G.T. (1913) Description of a new species of Meteorus (Braconidae). Entomologist, 46, 244-245.

Maeto, K. (1986a) Systematic studies on the tribe Meteorini from Japan (Hymenoptera, Braconidae), I, the genus Zele Curtis.

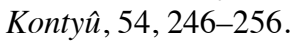

Maeto, K. (1986b) Systematic studies on the tribe Meteorini from Japan (Hymenoptera, Braconidae), II, the corax group of the genus Meteorus Haliday. Kontyû, 54, 405-413.

Maeto, K. (1988a) Systematic studies on the tribe Meteorini from Japan (Hymenoptera, Braconidae), III, the hirsutipes group. Kontyû, 56, 321-329.

Maeto, K. (1988b) Systematic studies on the tribe Meteorini from Japan (Hymenoptera, Braconidae), IV, the groups of Meteorus albizonalis and M. micropterus. Kontyû, 56, 581-589.

Maeto, K. (1989a) Systematic studies on the tribe Meteorini from Japan (Hymenoptera, Braconidae), V, the pulchricornis group of the genus Meteorus (1). Japanese Journal of Entomology, 57, 581-595.

Maeto, K. (1989b) Systematic studies on the tribe Meteorini from Japan (Hymenoptera, Braconidae), VI, the pulchricornis group of the genus Meteorus (2). Japanese Journal of Entomology, 57, 768-777.

Maeto, K. (1990a) Phylogenetic relationships and host associations of the subfamily Meteorinae Cresson (Hymenoptera: Braconidae). Japanese Journal of Entomology, 58, 383-396.

Maeto, K. (1990b) Systematic studies on the tribe Meteorini (Hymenoptera, Braconidae) from Japan, VII, The groups of Meteorus ictericus and M. rubens. Japanese Journal of Entomology, 58, 81-94.

Marshall, T.A. (1887) Monograph of British Braconidae, Part II. Transactions of the Royal Entomological Society of London, $1887,51-131$.

Marshall, T.A. (1891) In André. E., Spécies hymenoptères d'Europe \& d'Algérie. Rédigé d'après les principales collections, les mémoires les plus récents des auteurs et les communications des entomologistes spécialistes, 5, 635 pp. Beune (Coted'Or).

Marshall, T.A. (1897-1900) In André. E., Spécies hymenoptères d'Europe \& d'Algérie. Rédigé d'après les principales collections, les mémoires les plus récents des auteurs et les communications des entomologistes spécialistes, 5 , bis 373 pp. Beune (Cote-d'Or).

Martikainen, P., Koponen, M. (2001) Meteorus corax Marshall, 1898 (Hymenoptera: Braconidae), a new species to Finland and Russian Karelia, with an overview of northern species of Meteorus parasitizing beetles. Entomologica Fennica, 12, 169172.

Mason, W.R.M. (1973) Recognition of Zemiotes (Hymenoptera: Braconidae). Proceedings of the Entomological Society of Washington, 75, 213-215.

Morley, C. (1912) Rediscovery of the braconid Meteorus vexator (Hal.), with a description of the male. Entomologist, 45, 4-5.

Minamikawa, J. (1954) On the hymenopterous parasites of the tea-leafrollers found in Japan and Formosa. Mushi, 26, 35-46.

Muesebeck, C.F.W. (1923) A Revision of the North American species of ichneumon-flies belonging to the genus Meteorus Haliday. Proceedings of the U.S. National Museum, 63, 1-44.

Muesebeck, C.F.W. (1939) Five new species of Meteorus (Hymenoptera: Braconidae) Proceedings of the Entomological Society of Washington, 41, 83-87.

Nees von Esenbeck (1811) Ichneumonides adsciti, in genera et familias divisi. Magazin Gesellschaft Naturforschender Freunde in Berlin, 5.

Nees von Esenbeck, (1834) Hymenopterorum Ichneumonibus affinium monographiae, genera Europaea et species illustrantes, 1,320 pp Stuttgart, Tübingen, Cotta.

Nylander, J.A.A. (2004a) MrAIC.pl. Program distributed by the author. Evolutionary Biology Centre, Uppsala University.

Nylander, J.A.A. (2004b) MrModeltest v2. Program distributed by the autor. Evolutionary Biology Centre, Uppsala University. Papp, J. (1973) Contributions to the braconid funa of Yugoslavia (Hymenoptera, Braconidae) I. Acta Musei Macedonici Scien- 
tarium Naturalium, 14, 1-21.

Papp, J. (1983) A survey of the Braconid Fauna of the Hortobágy National Park (Hymenoptera, Braconidae) II. The fauna of the Hortobágy National Park, 2, 315-337.

Papp, J. (1994) A revision of the Braconidae (Hymenoptera) species described by J. W. Zetterstedt. Entomologica scandinavica, 25, 303-310.

Parker, H.L. (1931) Notes on Meteorus (Zemiotes nigricollis) Thomson, an occasional parasite of the European corn borer. Proceedings of the Entomological Society of Washington, 33, 93-103.

Parrott, A.W. (1955) Records of some important braconid parasites in New Zealand. New Zealand Entomologist, $1,16-22$.

Pitz, K.M., Dowling, A.P.G., Sharanowski, B.J., Boring, C.A., Seltmann K.C., Sharkey, M.J. (2007). Phylogenetic relationships among the Braconidae (Hymenoptera: Ichneumonoidea): A reassessment of Shi et al. (2005). Molecular Phylogenetics and Evolution, 43, 338-343.

Pons, J., Barraclough, T.G., Gomez-Zurita, J., Cardoso, A., Duran, D.P., Hazell, S., Kamoun, S., Sumlin, W.D. \& Vogler, A.P. (2006) Sequence-based species delimitation for the DNA taxonomy of undescribed insects. Systematic Biology, 55, 595609.

Quicke, D.L.J., (2001) In: Melika, G., Thuroczy, C. (eds), Parasitic wasps. Parasitic wasp taxonomy into the $21^{\text {st }}$ century, Budapest, 3-10 pp.

Quicke, D.L.J., Mori, J., Zaldivar-Riverón, A., Laurenne, N.M., Shaw, M.R. (2006) Suspended mummies in Aleiodes species (Hymenoptera: Braconidae: Rogadinae) with descriptions of six new species from western Uganda based largely on DNA sequence data. Journal of Natural History, 40, 2663-2680.

Ratzeburg, J.T.C. (1844) Die Ichneumonen der Forstinsecten in forstlicher und entomologischer Beziehung. Ein Anhang zur Abbildung und Beschreibung der Forstinsecten, 1, 224 pp.

Ratzeburg, J.T.C. (1848) Die Ichneumonen der Forstinsecten in forstlicher und entomologischer Beziehung. Ein anhang zur Abbildung und Beschreibung der Forstinsecten, 2, 238 pp. Berlin.

Ratzeburg, J.T.C. (1852) Die Ichneumonen der Forstinsecten in forstlicher und entomologischer Beziehung. Ein anhang zur Abbildung und Beschreibung der Forstinsecten 3, 272 pp.

Rokas, A., Nylander, J.A.A., Ronquist, F. \& Stone, G.N. (2002) A maximum likeliehood analysis of eight phylogenetic markers in gallwasps (Hymenoptera: Cynipidae): implication for insect phylogenetic studies. Molecular Phylogenetics and Evolution, 22, 206-219.

Roman, J.F. (1917) Braconiden aus den Färöern. Arkiv för Zoologi, 11, 1-10.

Ruthe, J.F. (1859) Verzeichniss der von Dr Staudinger im Jahre 1856 auf Island gesammelten Hymenopteren. Stettiner entomologische Zeitung, 20, 305-322.

Ruthe, J.F. (1862) Deutsche Braconiden. Berlin Entomologishe Zeitschrift, 6, 1-58.

Rösel von Rosenhof, A.J. (1749) Der monatlich herausgegebenen Insecten-Belustigung (Band2): ... welcher acht Classen verschiedener sowohl inländischer, als auch einiger ausländischer Insecte enthält. Nürnberg. Digitally available at Universitätsbibliothek Heidelberg (http://digi.ub.uni-heidelberg.de/diglit/roesel1749bd2/0339).

Schmiedeknecht, H.L.O. (1897) Die Braconiden-Gattung Meteorus Haliday. Illustrierte Wochenschrift für Entomologie, 2.

Schönwiese, F. (1934) Beobachtungen und Versuche anläßlich einer Übervermehrung von Lophyrus sertifer Geoffroy (rufus Panzer.) in Südkärnten in den Jahren 1931-1932. Zeitschrift für Angewandte Entomologie, 21, 463-500.

Shaw, S.R. (1985) A phylogenic study of the subfamilies Meteorinae and Euphorinae (Hymenoptera: Braconidae) Entomography, 3, 277-370.

Shaw, S.R. (1988) Meteorus brevicauda Thomsom (Hymenoptera: Braconidae) reared from larvae of Zeugophora subspinosa (Fabricius) (Coleoptera: Chrysomelidae) Entomologist's Gazette, 39, 205-206.

Shaw, S.R. \& Huddleston, T. (1991) Classification and Biology of Braconid Wasps (Hymenoptera: Braconidae) Handbooks for the Identification of British Insects, 7, 1-126.

Shaw, S.R. \& Jones, G.Z. (2009) A new species of solitary Meteorus (Hymenoptera: Braconidae) reared from caterpillars of toxic butterflies (Lepidoptera: Nymphalidae) in Ecuador. Journal of insect science, 9, 1-8.

Shenefelt, R.D. (1969) Hymenopterorum catalogus part 4 Braconidae, 1, 1-176. Junk, S-Gravenhage.

Shi, M., Chen, X.X. \& Achterberg, C. van. (2005) Phylogenetic relationships among the Braconidae (Hymenoptera: Ichneumonoidea) inferred from partial 16S rDNA, 28S rDNA D2, 18S rDNA gene sequences and morphological characters. Molecular Phylogenetics and Evolution, 37, 104-116.

Smith, M.A., Rodriguez, J.J., Whitfeld, J.B., Deans, A.R., Janzen, D.H., Hallwachs, W. \& Hebert, P.D.N. (2008) Extreme diversity of tropical parasitoid wasps exposed by iterative integration of natural history, DNA barcoding, morphology and collections. Proceedings of the National Academy of Sciences, 105, 12359-12364.

Spinola, M. (1808) Insectorum Liguriae species novae aut rariores, quas in agro Ligustico nuper detexit, descripsit, et iconibus illustravit (Hymenoptera), 2, 262 pp. Genuae.

Staden, R (1996) The Staden sequence analysis package. Molecular Biotechnology, 5, 233-341

Stigenberg, J., Vikberg, V. \& Belokobylskij, S.A. (2011) Meteorus acerbiavorus sp. nov. (Hymenoptera, Braconidae), a gregarious parasitoid of Acerbia alpina (Quensel) (Lepidoptera, Arctiidae) in North Finland. Journal of Natural History, 45, (2124), 1275-1294.

Szépligeti, G.V. (1896) Beiträge zur kenntnis der Ungarischen Braconiden. Természetrajzi Füzetek, 19, 359-386.

Tamura, K., Nei, M. \& Kumar, S. (2004) Prospects for inferring very large phylogenies by using the neighbor-joining method. 
Proceedings of the National Academy of Sciences (USA), 101, 11030-11035.

Tamura, K., Dudley, J., Nei, M. \& Kumar, S. (2007) MEGA4: Molecular Evolutionary Genetics Analysis (MEGA) software version 4.0. Molecular Biology and Evolution, 24, 1596-1599.

Telenga, N.A. (1950) Neue Arten parasitischer Hymenopteren der Familie Braconidae in der UdSSR. [in Russian]. Nauchnye Trudy Instituta Entomologii i Fitopatologii AN Ukraine SSR, 2, 293-308.

Thomson, C.G (1895) Bidrag till Braconidernas kännedom. Opuscula Entomologica, 20, 2141-2339.

Thunberg, C.P. (1822) Ichneumonoidea, insecta Hymenoptera, illustrate. Mémoires de l'Académie Impériale des Sciences de St. Pétersbourg, 8, 249-281.

Tobias, V.I (1966) Generic groupings and evolution of parasitic Hymenoptera of the subfamily Euphorinae (Hymenoptera, Braconidae). II. Entomological Review (Washington), 45, 348-358

Tobias, V.I (1986) Subfamily Euphorinae. In: Medvedev, G. S. (Ed.), Keys to the insects of the European part of U.S.S.R, 3 , 181-250. Hymenoptera, part 4: Nauka Publisher, Leningrad.

Tobias, V.I. (2000) Meteorini and Zele. In: Ler, P.A. Key to the insects of Russian Far East, 4, 198-226. Neuropteroidea, Mecoptera, Hymenoptera, Part 4. Vladivostok: Dal'nauka. [In Russian].

Viereck, H.L. (1912) Contributions to our knowledge of bees and ichneumon-flies, including the descriptions of twenty-one new genera and fifty-seven new species of ichneumon-flies. Proceedings of the United States National Museum, 42, 613648.

Watanabe, C. (1939a) Meteorus japonicus Ashmead, a parasite of the Gypsy Moth Lymantria dispar Linné (Hymenoptera: Braconidae). Insecta matsumurana, 13, 63-65.

Watanabe, C. (1939b) A new species of Meteorus bred from Camptoloma interiorata Walker (Hymenoptera: Braconidae) Insecta matsumurana, 14, 25-26.

Watanabe, C. (1951) A new species of Meteorus Haliday from Japan (Hymenoptera: Braconidae). Mushi, 22, (6) 45-46.

Wesmael, C. (1835) Monographie des Braconides de Belgique. Nouveaux Mémoires de l'Academie Royales des Science. Bruxelles, 9, 1-252.

Wesmael, C. (1838) Monographie des Braconides de Belgique IV. Nouveaux Mémoires de l'Academie Royales des Science. Bruxelles, 11, 1-166.

Yu, D.S., Achterberg, C. van. (Braconidae) \& Horstmann, K. (Ichneumonidae). 2005. World Ichneumonoidea 2004 - Taxonomy, Biology, Morphology and Distribution. DVD/CD. Taxapad. Vancouver, Canada. www.taxapad.com.

Zaldivar-Riverón, A., Mori, M. \& Quicke, D.L.J. (2006) Systematics of the cyclostome subfamilies of braconid parasitic wasps (Hymenoptera: Ichneumonidea): A simultaneous molecular and morphological Bayesian approach. Molecular Phylogenetics and Evolution, 38, 130-145.

Zetterstedt, J.W. (1838) Braconidaae. Pp. 397-407, In: Insecta Lapponica. Lipsiae.

Zitani, N.M. (2003) The evolution and adaptive significance of silk use in the Meteorinae (Hymenoptera, Braconidae). Unpublished PhD. Dissertation, University of Wyoming, 126 pp. 


\section{Appendix I}

\section{List of species, with synonyms}

\author{
Meteorus \\ abdominator (Nees, 1811) \\ brevipesalis Shenefeldt, 1969 \\ brunnipes (Ruthe, 1862) \\ abscissus Thomson, 1859 \\ affinis (Wesmael, 1385) \\ gracilis Ruthe, 1862 \\ punctiventris Ruthe, 1862 \\ ruthei Schmiedeknecht, 1897 \\ voloscensis Fisher, 1959 \\ alborossicus Lobodenko, 2000 \\ acerbiavorus Belokobylskij et al., 2011 \\ angiclypealis van Achterberg, 2001 \\ artocercus Stigenberg sp. nov. \\ breviantennatus Tobias, 1986 \\ brevicauda Thomson, 1895 \\ mongolicus Fahringer, 1935 \\ thuringiacus Schmiedeknecht, 1897 \\ cespitator (Thunberg, 1824) \\ ambiguus Ruthe, 1862 \\ atrator (Curtis, 1832) \\ humeralis (Zetterstedt, 1838) \\ rufipes (Zetterstedt, 1838) \\ similator (Nees, 1834) \\ cinctellus (Spinola, 1808) \\ fuscipes (Wesmael, 1835) \\ necator (Trentepohl, 1829) \\ tenellus Marshall, 1887 \\ cis (Bouché, 1834) \\ profligator Haliday, 1835 \\ colon (Haliday, 1835) \\ fasciatus (Ratzeburg, 1844) \\ fragilis (Wesmael, 1835) \\ luridus Ruthe, 1862 \\ consimilis (Nees, 1834) \\ albicornis Ruthe, 1862 \\ brevipes (Wesmael, 1835) \\ corax Marshall, 1898 \\ monochami Fischer, 1957 \\ densipilosus Stigenberg sp. nov. \\ eadyi Huddleston, 1980 \\ eklundi Stigenberg sp. nov. \\ filator (Haliday, 1835) \\ laticeps (Wesmael, 1835) \\ hodisensis Fischer, 1970 \\ heliophilus Fisher, 1970 \\ hirsutipes Huddleston, 1980 \\ ictericus (Nees, 1811) \\ adoxophyesi Minamikawa, 1954
}

confinis Ruthe, 1862

consors Ruthe, 1862

crassicrus Thomson, 1895

ephippium (Curtis, 1832)

fallax Ruthe, 1862

liquis Ruthe, 1862

lophyriphagus Fahringer, 1934

minutor (Thunberg, 1822)

pleuralis Ruthe, 1862

xanthomelas (Wesmael, 1835)

ipidivorus Tobias, 1986

jaculator (Haliday, 1835)

obscurellus Ruthe, 1862

tenuicornis Thomson, 1895

kyushuensis Maeto, 1988

limbatus Maeto, 1989

lionotus Thomson, 1895

ruficoloratus Fischer, 1957

longicaudis (Ratzeburg, 1884)

longipilosus Stigenberg sp. nov.

melanostictus Capron, 1887

monachae Tobias, 1986

niger Lyle, 1913

micropilosus Tobias, 1986

micropterus (Haliday, 1835)

nixoni Huddleston, 1980

obfuscatus (Nees, 1811)

fodori Papp, 1973

formosus (Wesmael, 1835)

thoracicus (Curtis, 1832)

obsoletus (Wesmael, 1835)

viridanae Johansson, 1964

oculatus Ruthe, 1862

pachypus Schmiedeknecht, 1897

pendulus (Müller, 1776)

gyrator (Thunberg, 1822)

parvulus Thomson, 1895

scutellator (Nees, 1834)

politutele Shenefelt, 1969

politus Telenga, 1950

pulchricornis (Wesmael, 1835)

baicalensis Telenga, 1950

graeffei Fischer, 1957

japonicus Ashmead, 1906

macedonicus Fischer, 1957

nipponensis Viereck, 1912

striatus Thomson, 1885

thomsoni Marshall, 1899

tuberculifer Fischer, 1957 
punctifrons Thomson, 1895

varinervis Tobias syn.n

radialis Tobias, 1986

rubens (Nees, 1811)

heteroneurus Thomson, 1895

islandicus Ruthe, 1859

leviventris (Wesmael, 1835)

medianus Ruthe, 1862

mesopotamicus Fischer, 1957

scutatus Costa, 1884

szechuanensis Fahringer, 1935

ruficeps (Nees, 1834)

nigritarsis Ruthe, 1862

pallipes (Wesmael, 1835)

rufus (De Geer,1778)

chinensis (Holmgren, 1868)

unicolor (Wesmael, 1835)

salicorniae Schmiedeknecht, 1897

ocellatus Watanabe, 1951

sibyllae Stigenberg sp.nov

stenomastax Stigenberg sp.nov

subjaculator Tobias, 1986

subtilisulcus Stigenberg sp.nov

sulcatus Szépligeti, 1896

insignis Muesebeck, 1939

molorchi Fischer, 1966

tabidus (Wesmael, 1835)

fascialis Ruthe, 1862

dubius Ruthe, 1862

pentheri Fischer, 1970

tenellus Marshall sp reinst.

boreus Tobias syn.n

versicolor (Wesmael, 1835)

bimaculatus (Wesmael, 1835)

brevicornis (Ratzeburg, 1844)

camptolomae Watanabe, 1939 decoloratus Ruthe, 1862

hartigi Shenefelt, 1969

ikonomovi Fischer, 1959

rugator (Ratzeburg, 1852)

unicolor (Hartig, 1838)

vexator (Haliday, 1935)

Zele

albiditarsus Curtis, 1832

albitarsus (Nees, 1834)

albitarsis (Haliday, 1835)

calcitrator (Curtis, 1837)

dispar (Wesmael, 1835)

maximus (Muesebeck, 1923)

pallitarsis (Cresson, 1872)

testaceator Curtis, 1832

testatrix (Schulz, 1906)

wesmaeli (Boie, 1850)

annulicrus (Thomson, 1895)

caligatus (Haliday, 1835)

alaskensis (Ashmead, 1902)

neesii (Ruthe, 1862)

sibiricus (Fahringer, 1930)

chlorophthalmus (Spinola, 1808)

chrysophthalamus (Nees, 1811)

nigricollis (Thomson, 1895)

nudator (Thunberg, 1822)

pallidus (Nees, 1812)

splendens (Costa, 1884)

deceptor (Wesmael. 1835)

maximus (Muesebeck, 1923)

pallitarsis (Cresson, 1872)

reticulates (Muesebeck, 1923)

romani (Fahringer, 1930)

rufulus (Thomson, 1895)

separandus (Fischer, 1957) 


\section{Appendix II}

Voucher specimens and GenBank accession numbers. Specimens with a asterix (*) was previously used in Stigenberg et al. 2011.

\begin{tabular}{|c|c|c|c|c|c|c|c|}
\hline Genus & Species & Sex & Type & DNA Nr & NHRS HYME ID & GenBank COI & GenBank 28S \\
\hline Meteorus & artocercus & female & Paratype & 1 & ethanol coll. & HQ263896 & HQ263720 \\
\hline Meteorus* & artocercus & female & Paratype & 2 & 000005177 & HQ263897 & HQ263721 \\
\hline Meteorus & tenellus & female & & 3 & 000005166 & HQ263898 & HQ263722 \\
\hline Meteorus & tenellus & female & & 4 & $*$ & HQ263899 & HQ263723 \\
\hline Meteorus* & tenellus & female & & 5 & 000005073 & HQ263900 & HQ263724 \\
\hline Meteorus & tenellus & female & & 6 & 000005174 & HQ263901 & HQ263725 \\
\hline Meteorus & densipilosus & female & Holotype & 7 & 000004936 & HQ263902 & HQ263726 \\
\hline Meteorus & eklundi & female & Holotype & 8 & 000004937 & HQ263903 & HQ263727 \\
\hline Meteorus & hirsutipes & female & & 9 & 000004944 & HQ263904 & HQ263728 \\
\hline Meteorus* & limbatus & female & & 10 & 000004891 & HQ263905 & HQ263729 \\
\hline Meteorus & kyushuensis & female & & 11 & 000004975 & HQ263906 & HQ263730 \\
\hline Meteorus & kyushuensis & female & & 12 & 000004974 & HQ263907 & HQ263731 \\
\hline Meteorus* & obsoletus & female & & 13 & 000005007 & HQ263908 & HQ263732 \\
\hline Meteorus & tenellus & female & & 14 & 000005074 & HQ263909 & HQ263733 \\
\hline Meteorus* & pulchricornis & female & & 15 & 000005027 & HQ263910 & HQ263734 \\
\hline Meteorus & stenomastax & female & & 16 & 000005051 & HQ263911 & HQ263735 \\
\hline Meteorus* & stenomastax & female & & 17 & 000005052 & HQ263912 & HQ263736 \\
\hline Meteorus* & tabidus & female & & 18 & 000005058 & HQ263913 & HQ263737 \\
\hline Meteorus* & tenellus & female & & 19 & 000005170 & HQ263914 & HQ263738 \\
\hline Meteorus* & tenellus & female & & 20 & 000005075 & HQ263915 & HQ263739 \\
\hline Meteorus* & versicolor & female & & 21 & 000005065 & HQ263916 & HQ263740 \\
\hline Meteorus* & obfuscatus & female & & 22 & 000004979 & HQ263917 & HQ263741 \\
\hline Meteorus & tenellus & female & & 23 & 000005168 & HQ263918 & HQ263742 \\
\hline Meteorus* & cinctellus & female & & 24 & 000005076 & HQ263919 & HQ263743 \\
\hline Meteorus & colon & female & & 25 & 000005077 & HQ263920 & HQ263744 \\
\hline Meteorus & abdominator & female & & 26 & 000005078 & HQ263921 & HQ263745 \\
\hline Meteorus & affinis & female & & 27 & 000005079 & HQ263922 & HQ263746 \\
\hline Meteorus & eklundi & female & Paratype & 28 & 000004939 & HQ263923 & HQ263747 \\
\hline Meteorus & cis & female & & 30 & 000005080 & HQ263924 & HQ263748 \\
\hline Meteorus & colon & female & & 31 & 000005081 & HQ263925 & HQ263749 \\
\hline Meteorus & consimilis & female & & 32 & 000005082 & HQ263926 & HQ263750 \\
\hline Meteorus & filator & female & & 33 & 000005083 & HQ263927 & HQ263751 \\
\hline Meteorus & ictericus & female & & 34 & 000005084 & HQ263928 & HQ263752 \\
\hline Meteorus & jaculator & female & & 35 & 000005085 & HQ263929 & HQ263753 \\
\hline Meteorus & micropterus & female & & 36 & 000005086 & HQ263930 & HQ263754 \\
\hline Meteorus & oculatus & female & & 37 & 000005087 & HQ263931 & HQ263755 \\
\hline Meteorus & ruficeps & female & & 38 & 000005088 & HQ263932 & HQ263756 \\
\hline Meteorus & pendulus & female & & 39 & 000005116 & HQ263933 & HQ263757 \\
\hline Meteorus & rubens & female & & 40 & 000005117 & HQ263934 & HQ263758 \\
\hline Meteorus & vexator & female & & 41 & 000005118 & HQ263935 & HQ263759 \\
\hline Zele & albiditarsus & female & & 42 & 000005089 & HQ263936 & HQ263760 \\
\hline Zele & deceptor & female & & 43 & 000005090 & HQ263937 & HQ263761 \\
\hline Zele & chlorophthalmus & female & & 44 & 000005091 & HQ263938 & HQ263762 \\
\hline Meteorus & abdominator & female & & 45 & 000005092 & n.a & HQ263763 \\
\hline Meteorus & affinis & female & & 46 & 000005093 & HQ263939 & HQ263764 \\
\hline Meteorus & artocercus & female & Paratype & 47 & $*$ & HQ263940 & HQ263765 \\
\hline
\end{tabular}

...... continued on the next page 
Appendix II (continued)

\begin{tabular}{|c|c|c|c|c|c|c|c|}
\hline Genus & Species & Sex & Type & DNA Nr & NHRS HYME ID & GenBank COI & GenBank 28S \\
\hline Meteorus & cespitator & female & & 48 & 000005094 & HQ263941 & HQ263766 \\
\hline Meteorus & cespitator & female & & 49 & 000005095 & HQ263942 & HQ263767 \\
\hline Meteorus & cinctellus & female & & 50 & 000004918 & HQ263943 & HQ263768 \\
\hline Meteorus & artocercus & female & & 51 & 000005073 & n.a & HQ263769 \\
\hline Meteorus & cinctellus & female & & 52 & 000004917 & HQ263944 & HQ263770 \\
\hline Meteorus* & cinctellus & female & & 53 & 000004916 & HQ263945 & HQ263771 \\
\hline Meteorus* & cinctellus & female & & 54 & 000004915 & HQ263946 & HQ263772 \\
\hline Meteorus & artocercus & female & Paratype & 55 & 000005182 & HQ263947 & HQ263773 \\
\hline Meteorus & artocercus & female & Paratype & 56 & 000005181 & HQ263948 & HQ263774 \\
\hline Meteorus & artocercus & female & Paratype & 57 & 000005180 & HQ263949 & HQ263775 \\
\hline Meteorus & artocercus & female & Paratype & 58 & 000005179 & HQ263950 & HQ263776 \\
\hline Meteorus & cis & female & & 59 & 000005096 & HQ263951 & HQ263777 \\
\hline Meteorus* & colon & female & & 60 & 000005097 & HQ263952 & HQ263778 \\
\hline Meteorus & colon & female & & 61 & 000005098 & n.a & HQ263779 \\
\hline Meteorus* & colon & female & & 62 & 000005099 & HQ263953 & HQ263780 \\
\hline Meteorus & abdominator & female & & 63 & 000005100 & HQ263954 & HQ263781 \\
\hline Meteorus & consimilis & female & & 64 & 000005101 & HQ263955 & HQ263782 \\
\hline Meteorus & ictericus & female & & 65 & 000005102 & HQ263956 & HQ263783 \\
\hline Meteorus & ictericus & female & & 66 & 000005103 & HQ263957 & HQ263784 \\
\hline Meteorus & ruficeps & female & & 67 & 000005104 & HQ263958 & HQ263785 \\
\hline Meteorus & jaculator & female & & 68 & 000005105 & HQ263959 & HQ263786 \\
\hline Meteorus* & micropterus & male & & 69 & 000005106 & HQ263960 & HQ263787 \\
\hline Meteorus* & micropterus & male & & 70 & 000005107 & HQ263961 & HQ263788 \\
\hline Meteorus* & ruficeps & female & & 71 & 000005048 & HQ263962 & HQ263789 \\
\hline Meteorus* & vexator & female & & 72 & 000005108 & HQ263963 & HQ263790 \\
\hline Zele & deceptor & female & & 73 & 000005109 & HQ263964 & HQ263791 \\
\hline Zele & albiditarsus & female & & 74 & 000005111 & HQ263965 & HQ263792 \\
\hline Zele & chlorophthalmus & female & & 75 & 000005110 & HQ263966 & HQ263793 \\
\hline Meteorus & tenellus & female & & 76 & 000005169 & HQ263967 & HQ263794 \\
\hline Meteorus & tenellus & female & & 77 & 000005171 & HQ263968 & HQ263795 \\
\hline Meteorus* & tenellus & female & & 78 & 000005172 & HQ263969 & HQ263796 \\
\hline Meteorus & tenellus & female & & 79 & 000005173 & HQ263970 & HQ263797 \\
\hline Meteorus & tenellus & female & & 80 & 000005167 & HQ263971 & HQ263798 \\
\hline Meteorus & tenellus & female & & 82 & 000005175 & HQ263972 & HQ263799 \\
\hline Zele & caligatus & female & & 84 & 000005112 & HQ263973 & HQ263800 \\
\hline Meteorus & longipilosus & female & Holotype & 85 & 000004978 & n.a & HQ263801 \\
\hline Meteorus & affinis & female & & 86 & 000005113 & HQ263974 & HQ263802 \\
\hline Meteorus & affinis & female & & 87 & 000005114 & HQ263975 & HQ263803 \\
\hline Meteorus & affinis & female & & 88 & 000005115 & HQ263976 & HQ263804 \\
\hline Meteorus & affinis & female & & 89 & 000005119 & HQ263977 & HQ263805 \\
\hline Meteorus & affinis & male & & 95 & 000005120 & HQ263978 & HQ263806 \\
\hline Meteorus & tabidus & male & & 96 & 000005121 & HQ263979 & HQ263807 \\
\hline Meteorus & tabidus & male & & 97 & 000005122 & HQ263980 & HQ263808 \\
\hline Meteorus & tenellus & male & & 99 & 000005124 & HQ263981 & HQ263809 \\
\hline Meteorus* & tenellus & male & & 100 & 000005125 & HQ263982 & HQ263810 \\
\hline Meteorus & sulcatus & female & & 101 & RMNH & HQ263983 & HQ263811 \\
\hline Meteorus & obfuscatus & female & & 102 & RMNH & HQ263984 & HQ263812 \\
\hline
\end{tabular}


Appendix II (continued)

\begin{tabular}{|c|c|c|c|c|c|c|c|}
\hline Genus & Species & Sex & Type & DNA Nr & NHRS HYME ID & GenBank COI & GenBank 28S \\
\hline Meteorus & longicaudis & female & & 103 & RMNH & HQ263985 & HQ263813 \\
\hline Meteorus* & abscissus & male & & 104 & 000005126 & HQ263986 & HQ263814 \\
\hline Meteorus* & tenellus & male & & 105 & 000005128 & HQ263987 & HQ263815 \\
\hline Meteorus & vexator & female & & 106 & 000005217 & HQ263988 & HQ263816 \\
\hline Meteorus & pendulus & female & & 107 & 000005127 & HQ263989 & HQ263817 \\
\hline Meteorus & pendulus & female & & 108 & 000005129 & HQ263990 & HQ263818 \\
\hline Meteorus & pendulus & female & & 109 & 000005130 & HQ263991 & HQ263819 \\
\hline Meteorus & pendulus & female & & 110 & 000005131 & HQ263992 & HQ263820 \\
\hline Meteorus & pendulus & female & & 111 & 000005132 & HQ263993 & HQ263821 \\
\hline Meteorus & pendulus & female & & 112 & 000005133 & HQ263994 & HQ263822 \\
\hline Meteorus & pendulus & female & & 113 & 000005134 & HQ263995 & HQ263823 \\
\hline Meteorus & colon & female & & 114 & 000005135 & HQ263996 & HQ263824 \\
\hline Meteorus & colon & female & & 115 & 000005136 & HQ263997 & HQ263825 \\
\hline Meteorus & colon & female & & 116 & 000005137 & HQ263998 & HQ263826 \\
\hline Meteorus & colon & female & & 117 & 000005138 & HQ263999 & HQ263827 \\
\hline Meteorus & colon & female & & 118 & 000005139 & HQ264000 & HQ263828 \\
\hline Meteorus & stenomastax & female & & 119 & * & HQ264001 & HQ263829 \\
\hline Meteorus* & rubens & female & & 120 & 000005140 & HQ264002 & HQ263830 \\
\hline Meteorus* & rubens & female & & 121 & 000005141 & HQ264003 & HQ263831 \\
\hline Meteorus* & rubens & male & & 122 & 000005142 & HQ264004 & HQ263832 \\
\hline Meteorus* & rubens & female & & 123 & 000005143 & HQ264005 & HQ263833 \\
\hline Meteorus & vexator & female & & 124 & 000005144 & HQ264006 & HQ263834 \\
\hline Meteorus & vexator & female & & 125 & 000005145 & HQ264007 & HQ263835 \\
\hline Meteorus & alborossicus & female & & 126 & 000005146 & $\mathrm{n} . \mathrm{a}$ & HQ263836 \\
\hline Meteorus & vexator & female & & 127 & 000005147 & HQ264008 & HQ263837 \\
\hline Meteorus* & versicolor & female & & 128 & 000005148 & HQ264009 & HQ263838 \\
\hline Meteorus* & pulchricornis & female & & 129 & 000005149 & HQ264010 & HQ263839 \\
\hline Meteorus* & versicolor & female & & 130 & 000005150 & HQ264011 & HQ263840 \\
\hline Meteorus* & versicolor & female & & 131 & 000005151 & HQ264012 & HQ263841 \\
\hline Meteorus & ictericus & female & & 132 & 000005153 & HQ264013 & HQ263842 \\
\hline Meteorus & ruficeps & female & & 133 & 000005154 & HQ264014 & HQ263843 \\
\hline Meteorus & ruficeps & female & & 134 & 000005152 & HQ264015 & HQ263844 \\
\hline Meteorus & ictericus & female & & 135 & 000005155 & HQ264016 & HQ263845 \\
\hline Meteorus & cespitator & female & & 136 & 000005161 & HQ264017 & HQ263846 \\
\hline Meteorus & cespitator & female & & 137 & 000005162 & HQ264018 & HQ263847 \\
\hline Meteorus & filator & female & & 138 & 000005156 & HQ264019 & HQ263848 \\
\hline Meteorus & filator & female & & 139 & 000005160 & HQ264020 & HQ263849 \\
\hline Meteorus & cis & female & & 140 & 000005157 & HQ264021 & HQ263850 \\
\hline Meteorus* & obfuscatus & female & & 141 & 000005158 & HQ264022 & HQ263851 \\
\hline Meteorus & pendulus & female & & 142 & 000005159 & HQ264023 & HQ263852 \\
\hline Meteorus & colon & female & & 143 & 000005163 & HQ264024 & HQ263853 \\
\hline Meteorus & longipilosus & female & Paratype & 144 & 000004977 & n.a & HQ263854 \\
\hline Meteorus & abdominator & female & & 145 & 000005184 & n.a & HQ263855 \\
\hline Meteorus & abdominator & female & & 146 & 000005185 & n.a & HQ263856 \\
\hline Meteorus & abdominator & female & & 147 & 000005186 & n.a & HQ263857 \\
\hline Meteorus & abdominator & female & & 148 & 000005187 & n.a & HQ263858 \\
\hline Meteorus & abdominator & female & & 149 & 000005188 & n.a & HQ263859 \\
\hline
\end{tabular}


Appendix II (continued)

\begin{tabular}{|c|c|c|c|c|c|c|c|}
\hline Genus & Species & Sex & Type & DNA Nr & NHRS HYME ID & GenBank COI & GenBank 28S \\
\hline Meteorus & affinis & female & & 150 & 000005189 & n.a & HQ263860 \\
\hline Meteorus & affinis & female & & 151 & 000005190 & n.a & HQ263861 \\
\hline Meteorus & affinis & male & & 152 & 000005191 & n.a & HQ263862 \\
\hline Meteorus & affinis & female & & 153 & 000005192 & HQ264025 & HQ263863 \\
\hline Meteorus & affinis & female & & 154 & 000005193 & HQ264026 & HQ263864 \\
\hline Meteorus & affinis & female & & 155 & 000005194 & HQ264027 & HQ263865 \\
\hline Meteorus & affinis & female & & 156 & 000005195 & HQ264029 & HQ263867 \\
\hline Meteorus & cis & female & & 157 & 000005196 & HQ264030 & HQ263868 \\
\hline Meteorus & cis & female & & 158 & 000005197 & HQ264031 & HQ263869 \\
\hline Meteorus & consimilis & female & & 159 & 000005198 & HQ264032 & HQ263870 \\
\hline Meteorus & consimilis & female & & 160 & 000005199 & n.a. & HQ263871 \\
\hline Meteorus & consimilis & female & & 161 & $*$ & HQ264033 & HQ263872 \\
\hline Meteorus* & abscissus & female & & 162 & 000005216 & HQ264048 & HQ263887 \\
\hline Meteorus* & rubens & female & & 163 & 000005212 & HQ264044 & HQ263883 \\
\hline Meteorus & consimilis & female & & 164 & 000005201 & HQ264028 & HQ263866 \\
\hline Meteorus & consimilis & male & & 165 & 000005200 & HQ264034 & HQ263873 \\
\hline Meteorus & filator & male & & 166 & 000005202 & HQ264035 & HQ263874 \\
\hline Meteorus & filator & male & & 167 & 000005203 & HQ264036 & HQ263875 \\
\hline Meteorus & cis & female & & 168 & 000005204 & HQ264037 & HQ263876 \\
\hline Meteorus & sibyllae & female & Paratype & 169 & 000005205 & HQ264038 & HQ263877 \\
\hline Meteorus & cis & female & & 171 & 000005207 & HQ264039 & HQ263878 \\
\hline Meteorus & sibyllae & female & Holotype & 172 & 000005208 & HQ264040 & HQ263879 \\
\hline Meteorus* & stenomastax & female & & 173 & 000005213 & HQ264045 & HQ263884 \\
\hline Meteorus & pendulus & female & & 174 & 000005209 & HQ264041 & HQ263880 \\
\hline Meteorus & pendulus & female & & 175 & 000005210 & HQ264042 & HQ263881 \\
\hline Meteorus & pendulus & female & & 176 & 000005211 & HQ264043 & HQ263882 \\
\hline Meteorus* & abscissus & female & & 177 & 000005214 & HQ264046 & HQ263885 \\
\hline Meteorus* & rubens & male & & 178 & 000005215 & HQ264047 & HQ263886 \\
\hline Meteorus* & acerbiavorus & female & Paratype & 179 & 000005223 & HQ264049 & HQ263888 \\
\hline Meteorus* & acerbiavorus & male & Paratype & 180 & 000005222 & HQ264050 & HQ263889 \\
\hline Meteorus & vexator & female & & 181 & $*$ & HQ264051 & HQ263890 \\
\hline Meteorus* & acerbiavorus & female & Paratype & 183 & 000005246 & n.a & HQ263891 \\
\hline Meteorus* & acerbiavorus & female & Paratype & 185 & 000005241 & HQ264052 & HQ263892 \\
\hline Meteorus & abscissus & female & & 186 & Ribes coll. & HQ264053 & HQ263893 \\
\hline Meteorus* & acerbiavorus & male & Paratype & 187 & 000005247 & HQ264054 & HQ263894 \\
\hline Meteorus* & acerbiavorus & male & Paratype & 188 & 000005245 & n.a & HQ263895 \\
\hline Meteorus & $\operatorname{corax}$ & - & & & $*$ & AY935418 & AY935488 \\
\hline Centistes & & - & & & AB071 & $\mathrm{n} . \mathrm{a}$ & n.a \\
\hline Leiophron & & - & & & AB022 & n.a & n.a \\
\hline Mannokeria & & - & & & AB086 & n.a & n.a \\
\hline Peristenus & & - & & & AB016 & n.a & $\mathrm{n} . \mathrm{a}$ \\
\hline Streblocera & & - & & & AB073 & n.a & n.a \\
\hline Syntretus & & - & & & JS111 & n.a & n.a \\
\hline
\end{tabular}

